\title{
Oxidative dehalogenation of perhalogenated benzenes by cytochrome P450 Compound I
}

John C Hackett, Toby T. Sanan, and Christopher M. Hadad*

Contribution from the Department of Chemistry, The Ohio State University, 100 W. $18^{\text {th }}$ Ave., Columbus, $\mathrm{OH} 43210$ 
Table S1. RI-PBE/TZVP and B3LYP/TZVP//RI-PBE/TZVP energetic spacings (eV) of the ${ }^{5} \mathrm{~A}_{1}$ and ${ }^{3} \mathrm{~A}_{2}$ states of $\left[\mathrm{FeO}\left(\mathrm{NH}_{3}\right)_{4}\left(\mathrm{H}_{2} \mathrm{O}\right)\right]^{2+}$.

\begin{tabular}{lll}
\hline & RI-PBE/TZVP & B3LYP/TZVP//RI-PBE/TZVP \\
\hline${ }^{5} \mathrm{~A}_{1}$ & 0.53 & 0.15 \\
${ }^{3} \mathrm{~A}_{2}$ & 0.00 & 0.00 \\
\hline
\end{tabular}




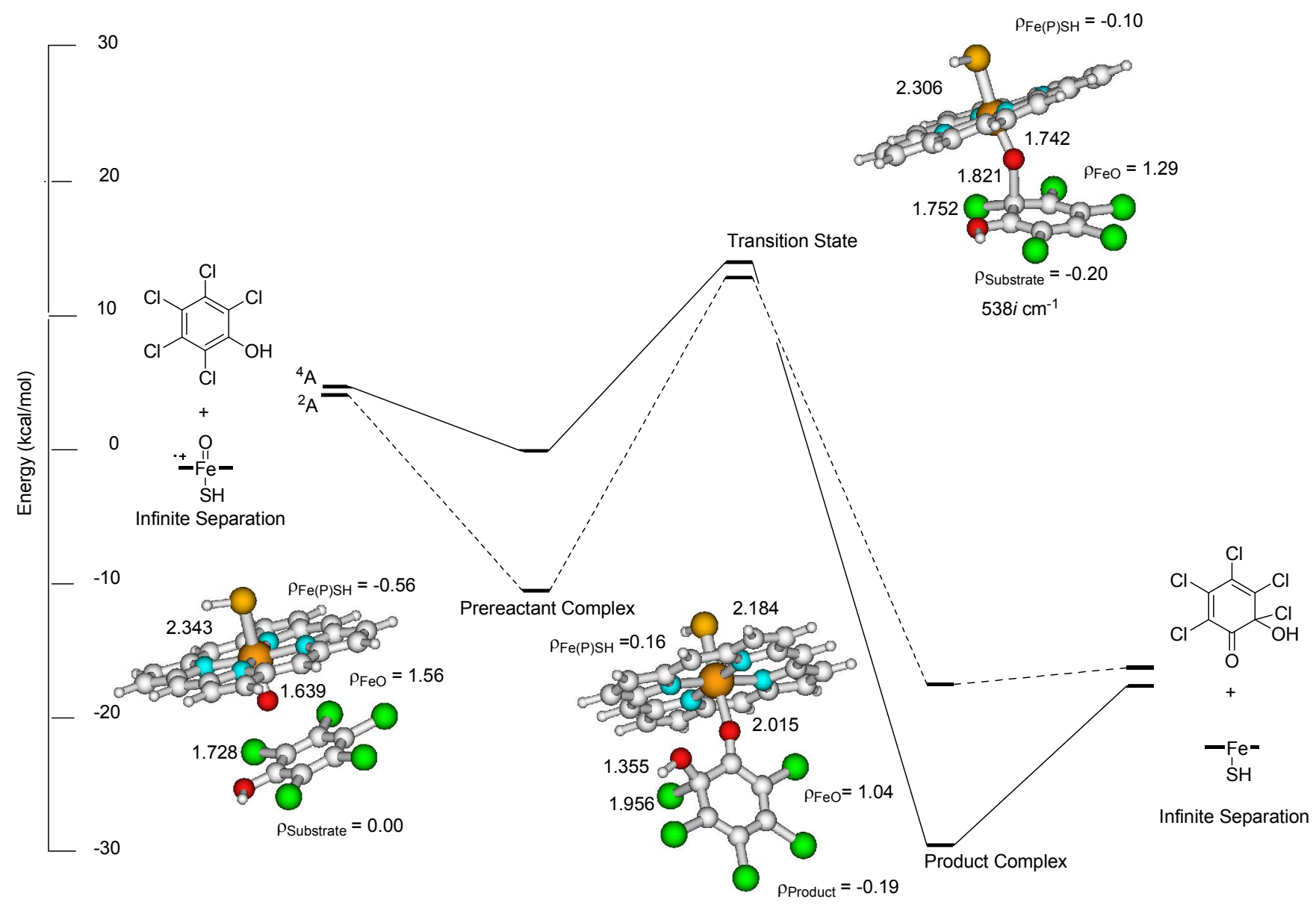

Figure S1. RI-PBE/TZVP,SV(P) relative energy diagram of stationary points along the reaction coordinate for model Compound I ortho-addition to pentachlorophenol. Geometries were optimized at the RI-PBE/TZVP,SV(P) level of theory. Bond distances are listed in Angstroms, and energies are in 
$\mathrm{kcal} / \mathrm{mol}$. Values corresponding to the quartet surface are in parentheses.

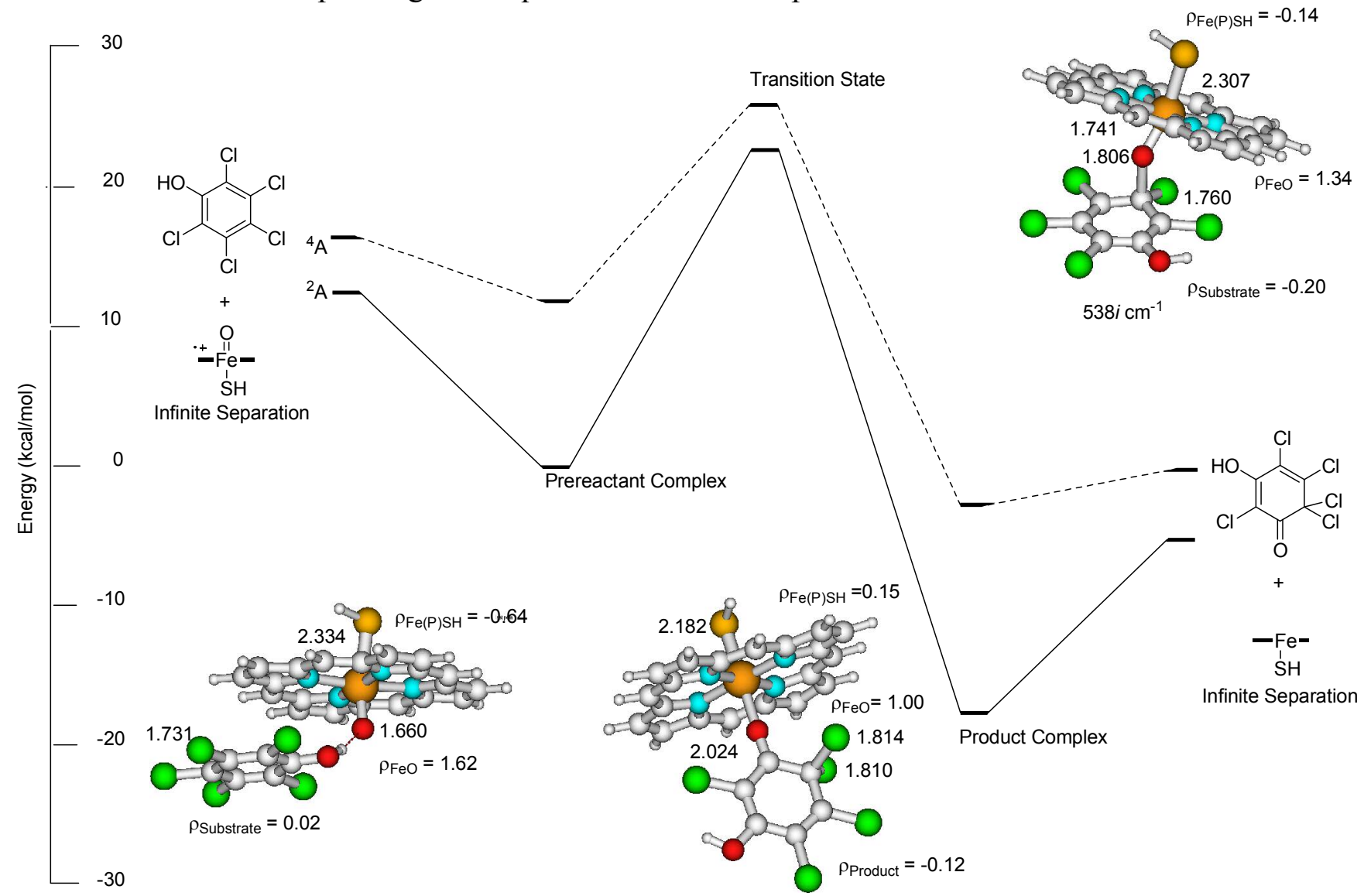

Figure S2. . RI-PBE/TZVP,SV(P) relative energy diagram of stationary points along the reaction coordinate for model Compound I meta-addition to pentachlorophenol. Bond distances are listed in Angstroms, and energies are in $\mathrm{kcal} / \mathrm{mol}$. Values corresponding to the quartet surface are in parentheses. 


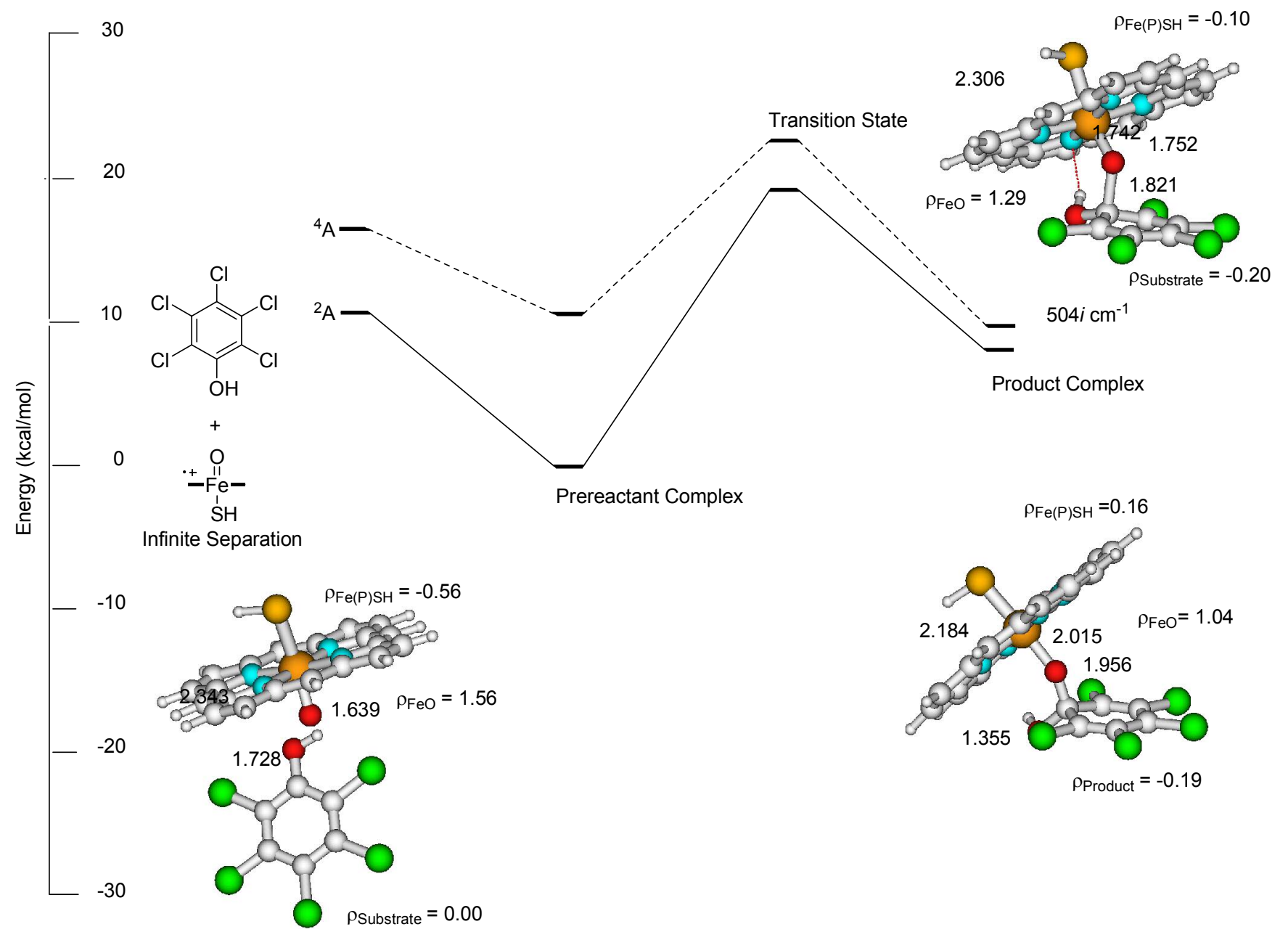

Figure S3. . RI-PBE/TZVP,SV(P) relative energy diagram of stationary points along the reaction coordinate for model Compound I ipso-addition to pentachlorophenol. Geometries Bond distances are listed in Angstroms, and energies are in $\mathrm{kcal} / \mathrm{mol}$. Values corresponding to the quartet surface are in parentheses. 


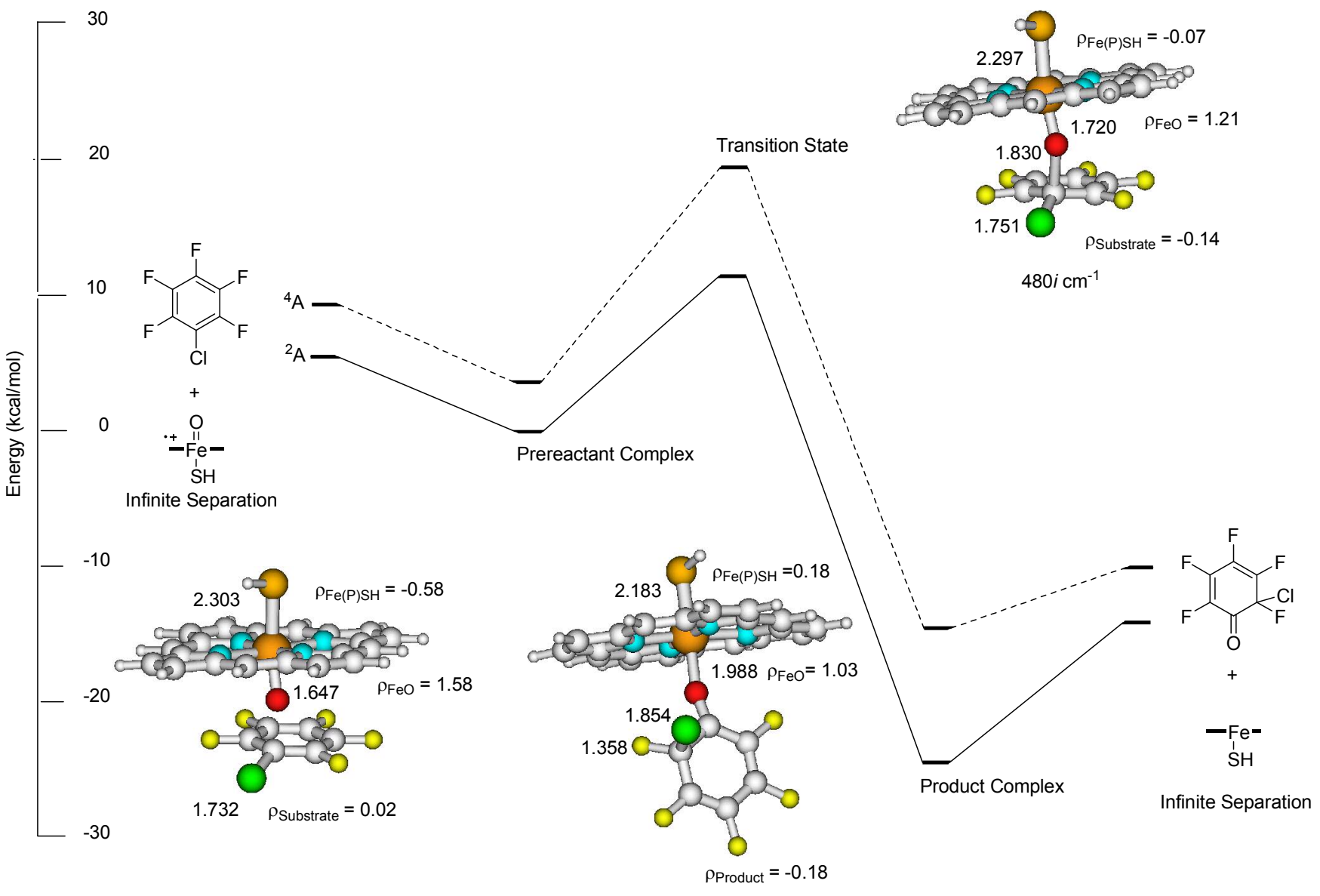

Figure S4. . RI-PBE/TZVP,SV(P) relative energy diagram of stationary points along the reaction coordinate for model Compound I $p$-Cl addition to chlorofluorobenzene. Bond distances are listed in Angstroms, and energies are in $\mathrm{kcal} / \mathrm{mol}$. Values corresponding to the quartet surface are in parentheses. 


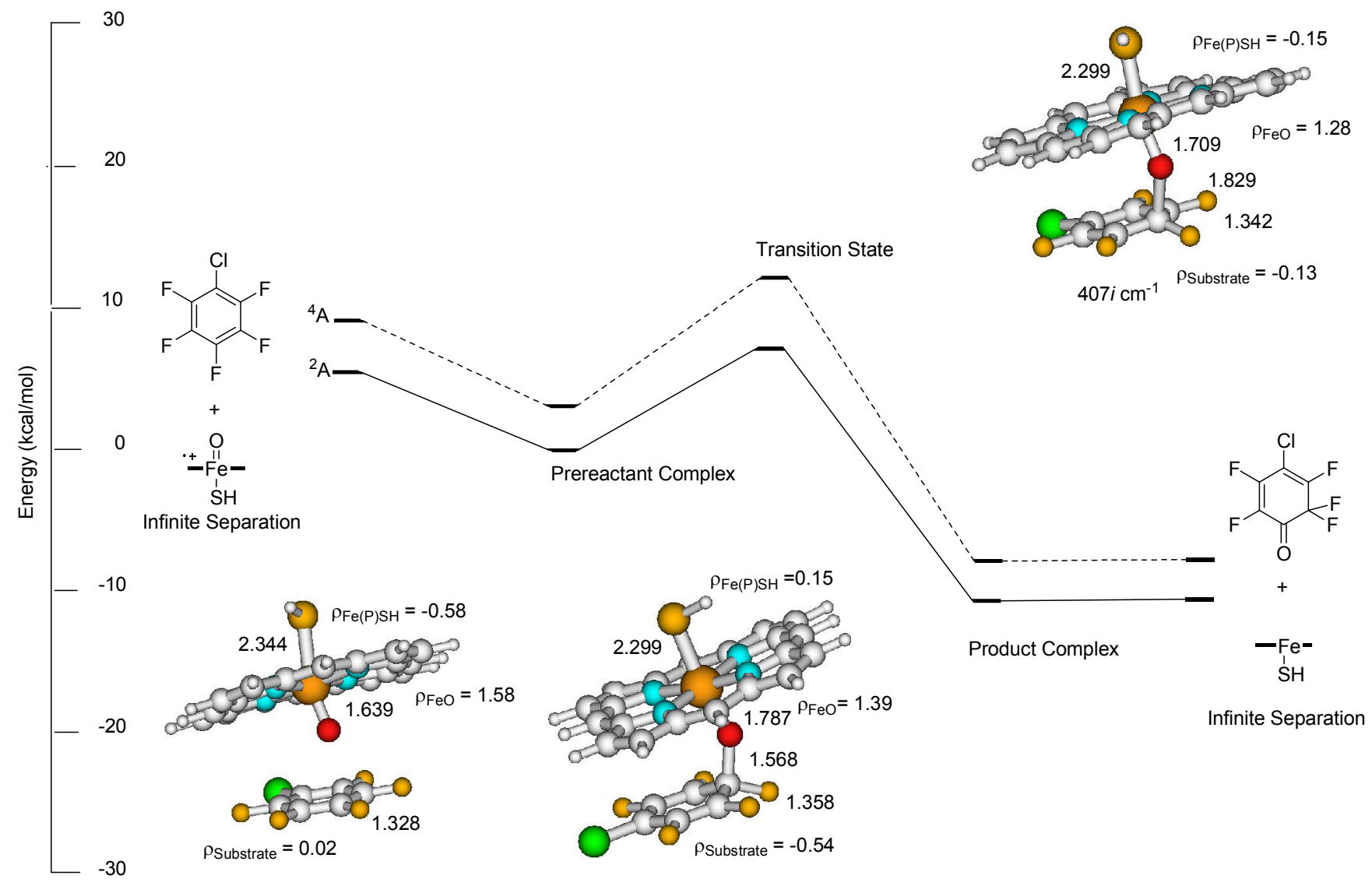

Figure S5. RI-PBE/TZVP,SV(P) relative energy diagram of stationary points along the reaction coordinate for model Compound I $p$-F addition to chloropentafluorobenzene. Bond distances are listed in Angstroms, and energies are in $\mathrm{kcal} / \mathrm{mol}$. Values corresponding to the quartet surface are in parentheses.

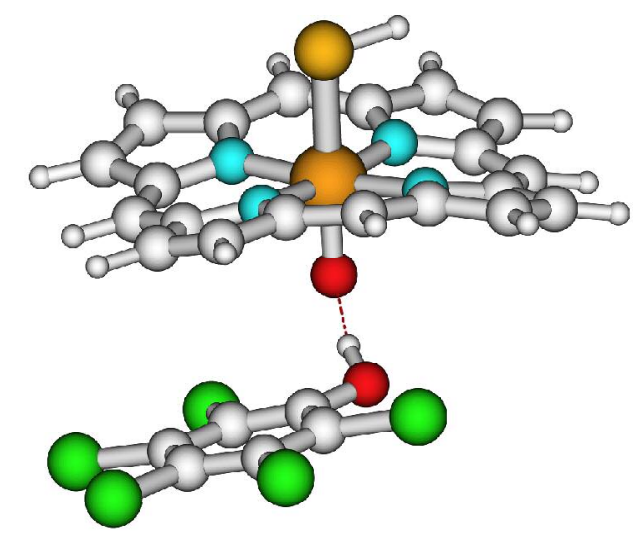

Figure S6. RI-PBE/TZVP,SV(P) optimized geometry of the pre-reactant complex on the quartet surface for the ortho oxidation of pentachlorophenol. 


\section{${ }^{2} \mathrm{~A}[($ Porphyrin $)(\mathrm{Fe}=\mathrm{O})(\mathrm{SH})$}

RI-PBE/TZVP, SV (P) Cartesian coordinates

40

Energy $=-2723.602087394$

\begin{tabular}{|c|c|c|c|}
\hline C & -0.6296958 & -2.9620430 & -0.1127171 \\
\hline $\mathrm{N}$ & 0.3424960 & -1.9860072 & -0.0883799 \\
\hline C & 1.5474656 & -2.6447619 & -0.0981552 \\
\hline C & 1.3401553 & -4.0781758 & -0.1116484 \\
\hline $\mathrm{C}$ & -0.0170706 & -4.2752868 & -0.1230079 \\
\hline $\mathrm{Fe}$ & 0.0500247 & 0.0286875 & -0.1748609 \\
\hline 0 & -0.1517532 & -0.0433769 & -1.7958099 \\
\hline C & 2.7982908 & -2.0232389 & -0.1050351 \\
\hline C & 3.0048152 & -0.6434639 & -0.1132446 \\
\hline $\mathrm{N}$ & 2.0178438 & 0.3211210 & -0.1194274 \\
\hline C & 2.6736483 & 1.5345156 & -0.0968542 \\
\hline $\mathrm{C}$ & 4.1065182 & 1.3322480 & -0.0854459 \\
\hline C & 4.3125644 & -0.0233084 & -0.0962701 \\
\hline C & 2.0616666 & 2.7875457 & -0.0740564 \\
\hline $\mathrm{C}$ & 0.6819011 & 3.0021462 & -0.0675944 \\
\hline N & -0.2743910 & 2.0127298 & -0.077157 \\
\hline $\mathrm{C}$ & -1.4922888 & 2.6605087 & -0.0953748 \\
\hline $\mathrm{C}$ & -1.2959591 & 4.0953810 & -0.0825014 \\
\hline C & 0.0588867 & 4.3085563 & -0.0629304 \\
\hline C & -2.7495419 & 2.0511353 & -0.1206998 \\
\hline $\mathrm{C}$ & -2.9782721 & 0.6740885 & -0.1267923 \\
\hline N & -1.9950031 & -0.2858859 & -0.0914765 \\
\hline $\mathrm{C}$ & -2.6376730 & -1.5024495 & -0.1306716 \\
\hline $\mathrm{C}$ & -4.0733570 & -1.3052915 & -0.1682691 \\
\hline $\mathrm{C}$ & -4.2860698 & 0.0499149 & -0.1654225 \\
\hline $\mathrm{C}$ & -2.0099481 & -2.7486383 & -0.1345563 \\
\hline$S$ & -0.1869318 & -0.1992080 & 2.147320 \\
\hline $\mathrm{H}$ & -0.8838039 & 0.9567507 & 2.397208 \\
\hline & -5.2389496 & 0.5955103 & -0.189096 \\
\hline $\mathrm{H}$ & -4.8140661 & -2.1157348 & -0.194467 \\
\hline $\mathrm{H}$ & -2.6559221 & -3.6393651 & -0.1631010 \\
\hline $\mathrm{H}$ & -0.5737272 & -5.2218440 & -0.1380457 \\
\hline $\mathrm{H}$ & 2.1439647 & -4.8263991 & -0.1157596 \\
\hline $\mathrm{H}$ & 3.6891156 & -2.6695073 & -0.1052112 \\
\hline $\mathrm{H}$ & 5.2623042 & -0.5745921 & -0.088464 \\
\hline & 4.8494394 & 2.1407542 & -0.067277 \\
\hline & 2.7153074 & 3.6728656 & -0.061532 \\
\hline & 0.6055148 & 5.2609244 & -0.051180 \\
\hline & -2.1079274 & 4.8348188 & -0.090029 \\
\hline & 292222 & 2.7124168 & -0.142868 \\
\hline
\end{tabular}

Vibrational frequencies

$\begin{array}{rrrrrr}0.00 & 0.00 & 0.00 & 0.00 & 0.00 & 0.00 \\ 39.22 & 51.29 & 91.03 & 98.01 & 101.33 & 130.01 \\ 145.27 & 151.10 & 171.17 & 205.64 & 217.39 & 232.94 \\ 237.80 & 271.58 & 273.42 & 277.24 & 278.84 & 286.06 \\ 300.64 & 307.29 & 346.25 & 358.41 & 359.29 & 364.38 \\ 396.87 & 400.81 & 418.58 & 419.64 & 420.21 & 421.92 \\ 459.99 & 599.81 & 650.15 & 656.82 & 657.29 & 673.02 \\ 680.15 & 694.50 & 695.31 & 697.98 & 715.93 & 723.91 \\ 734.13 & 735.28 & 764.09 & 772.02 & 772.45 & 778.44 \\ 782.38 & 784.76 & 784.90 & 798.64 & 841.34 & 850.45 \\ 851.18 & 855.88 & 859.66 & 887.14 & 892.49 & 892.66 \\ 894.75 & 993.38 & 1002.21 & 1002.65 & 1010.95 & 1015.04 \\ 1031.59 & 1032.26 & 1035.69 & 1041.07 & 1041.24 & 1042.17 \\ 1054.58 & 1140.71 & 1148.70 & 1149.29 & 1182.90 & 1183.69 \\ 1261.03 & 1262.52 & 1316.28 & 1329.59 & 1330.97 & 1340.99 \\ 1363.89 & 1376.01 & 1383.26 & 1391.51 & 1393.25 & 1470.13 \\ 1472.29 & 1473.12 & 1504.96 & 1525.32 & 1558.19 & 1558.59 \\ 1579.63 & 1604.72 & 1609.35 & 1611.08 & 1662.16 & 2545.86 \\ 3123.84 & 3124.11 & 3126.06 & 3126.16 & 3154.26 & 3154.87 \\ 3155.11 & 3155.34 & 3173.21 & 3173.85 & 3174.07 & 3174.32\end{array}$

TURBOMOLE Energies and Thermochemistry ${ }^{1}$

.00 -91
2.94 86.06 64.38 673.02 23.91 80.45 92.66 5.04 83.69 40.99 470.13
58.59 54.87 3174.32

\begin{tabular}{|c|c|c|}
\hline $\mathrm{RI}-\mathrm{PBE} / \mathrm{TZVP}, \mathrm{SV}(\mathrm{P})$ & & -2723.602087 \\
\hline $\mathrm{ZPE}$ & & 0.2803493 \\
\hline $\mathrm{RI}-\mathrm{PBE} / \mathrm{TZVP}, \mathrm{SV}(\mathrm{P})$ & $+\mathrm{ZPE}$ & -2723.321738 \\
\hline RI - PBE/TZVPP & & -2724.899848 \\
\hline B3 - LYP / TZVP , SV (P) & & -2724.424662 \\
\hline $\mathrm{ZPE} \quad(\mathrm{kJ} / \mathrm{mol})$ & & 736 \\
\hline $\ln ($ qtrans $)$ & & 19.64 \\
\hline $\ln ($ qrot $)$ & & 16.12 \\
\hline $\ln ($ qvib) & & 15.36 \\
\hline chem.pot. (kJ/mol) & & 609.33 \\
\hline energy (kJ/mol ) & & 792.19 \\
\hline entropy $(\mathrm{kJ} / \mathrm{mol} / \mathrm{K})$ & & 0.62163 \\
\hline $\mathrm{CV} \quad(\mathrm{kJ} / \mathrm{mol}-\mathrm{K})$ & & 0.3675535 \\
\hline $\mathrm{Cp} \quad((\mathrm{kJ} / \mathrm{mol}-\mathrm{K})$ & & 0.3758678 \\
\hline $\mathrm{RI}-\mathrm{PBE} / \mathrm{TZVP}, \mathrm{SV}(\mathrm{P})$ & $<S^{2}>$ & 1.10 \\
\hline $\mathrm{RI}-\mathrm{PBE} / \mathrm{TZVPP}<\mathrm{S}^{2}>$ & & 1.03 \\
\hline B3 - LYP / TZVP , SV (P) & $<S^{2}>$ & 1.46 \\
\hline
\end{tabular}




\section{${ }^{4} \mathrm{~A}[($ Porphyrin $)(\mathrm{Fe}=\mathrm{O})(\mathrm{SH})$}

RI-PBE/TZVP, SV (P) Cartesian coordinates

40

Energy $=-2723.594528869$

$\begin{array}{lrrr}\mathrm{C} & 1.3220657 & -2.7087746 & 0.0676101 \\ \mathrm{~N} & 1.4732550 & -1.3502404 & -0.0992487 \\ \mathrm{C} & 2.8152660 & -1.1313986 & -0.3087462 \\ \mathrm{C} & 3.5383431 & -2.3854032 & -0.2648509 \\ \mathrm{C} & 2.6110562 & -3.3651865 & -0.0124268 \\ \mathrm{Fe} & 0.0063679 & 0.0149872 & -0.1987373 \\ \mathrm{O} & 0.0222095 & 0.0018788 & -1.8624260 \\ \mathrm{C} & 3.4045509 & 0.1274509 & -0.4485098 \\ \mathrm{C} & 2.7293850 & 1.3443856 & -0.3063918 \\ \mathrm{~N} & 1.3779970 & 1.4816626 & -0.0957020 \\ \mathrm{C} & 1.1588287 & 2.8176865 & 0.1364731 \\ \mathrm{C} & 2.4084111 & 3.5498408 & 0.0824825 \\ \mathrm{C} & 3.3840850 & 2.6348471 & -0.2224920 \\ \mathrm{C} & -0.0971769 & 3.4085127 & 0.3005575 \\ \mathrm{C} & -1.3151430 & 2.7443969 & 0.1306306 \\ \mathrm{~N} & -1.4557534 & 1.3948280 & -0.0978771 \\ \mathrm{C} & -2.7961430 & 1.1837594 & -0.3290345 \\ \mathrm{C} & -3.5222607 & 2.4352774 & -0.2567632 \\ \mathrm{C} & -2.6028365 & 3.4037181 & 0.0593407 \\ \mathrm{C} & -3.3993587 & -0.0693136 & -0.4800067 \\ \mathrm{C} & -2.7408440 & -1.2903611 & -0.3191488 \\ \mathrm{~N} & -1.3883629 & -1.4268134 & -0.0986432 \\ \mathrm{C} & -1.1574901 & -2.7775240 & 0.0758514 \\ \mathrm{C} & -2.4066044 & -3.5057902 & -0.0000118 \\ \mathrm{C} & -3.3882871 & -2.5844843 & -0.2678384 \\ \mathrm{C} & 0.0993131 & -3.3762316 & 0.1822939 \\ \mathrm{~S} & -0.0015070 & -0.2505369 & 2.2298753 \\ \mathrm{H} & -1.3264945 & 0.0326486 & 2.4459120 \\ \mathrm{H} & -4.4653827 & -2.7506369 & -0.4030856 \\ \mathrm{H} & -2.5012455 & -4.5941749 & 0.1121315 \\ \mathrm{H} & 0.1296625 & -4.4710597 & 0.2920443 \\ \mathrm{H} & 2.7694354 & -4.4461045 & 0.0992641 \\ \mathrm{H} & 4.6237455 & -2.4864271 & -0.3980532 \\ \mathrm{H} & 4.4944281 & 0.1655491 & -0.5981363 \\ \mathrm{H} & 4.4619518 & 2.7990467 & -0.3536637 \\ \mathrm{H} & 2.5062582 & 4.6325895 & 0.2377492 \\ \mathrm{H} & -0.1301534 & 4.4951259 & 0.4732071 \\ \mathrm{H} & -2.7638402 & 4.4794630 & 0.2104346 \\ \mathrm{H} & -4.6058235 & 2.5388594 & -0.4028312 \\ \mathrm{H} & -4.4872588 & -0.0939105 & -0.6457373\end{array}$

Vibrational frequencies

$\begin{array}{rrrrrr}0.00 & 0.00 & 0.00 & 0.00 & 0.00 & 0.00 \\ 45.48 & 52.34 & 87.56 & 90.22 & 101.70 & 139.54 \\ 146.02 & 167.88 & 171.14 & 177.90 & 211.84 & 217.59 \\ 233.49 & 265.70 & 269.81 & 271.19 & 280.03 & 285.89 \\ 312.12 & 313.45 & 343.94 & 358.38 & 359.61 & 368.70 \\ 402.26 & 405.63 & 419.46 & 421.09 & 422.09 & 423.85 \\ 458.53 & 588.05 & 650.51 & 653.43 & 654.09 & 667.89 \\ 674.37 & 692.31 & 693.19 & 697.28 & 714.09 & 724.26 \\ 734.37 & 735.18 & 762.54 & 769.14 & 769.65 & 775.11 \\ 779.00 & 785.51 & 786.24 & 799.95 & 811.47 & 842.41 \\ 851.06 & 851.15 & 859.42 & 885.86 & 891.16 & 891.24 \\ 893.45 & 994.10 & 1003.75 & 1004.70 & 1011.08 & 1016.54 \\ 1031.38 & 1033.32 & 1037.30 & 1041.26 & 1041.83 & 1043.52 \\ 1055.85 & 1137.52 & 1146.72 & 1148.02 & 1181.14 & 1185.55 \\ 1261.62 & 1262.62 & 1297.39 & 1326.90 & 1327.89 & 1339.21 \\ 1363.83 & 1371.39 & 1381.01 & 1385.87 & 1395.76 & 1465.14 \\ 1468.16 & 1469.01 & 1500.93 & 1521.63 & 1553.94 & 1554.03 \\ 1573.65 & 1584.11 & 1602.61 & 1604.08 & 1654.97 & 2551.76 \\ 3124.45 & 3125.35 & 3125.44 & 3125.59 & 3155.49 & 3155.69 \\ 3155.90 & 3156.08 & 3174.29 & 3174.44 & 3174.73 & 3174.90\end{array}$

TURBOMOLE Energies and Thermochemistry ${ }^{1}$

\begin{tabular}{lr}
\hline RI-PBE/TZVP,SV(P) & -2723.594529 \\
\hline ZPE & 0.2798986 \\
RI-PBE/TZVP,SV(P) + ZPE & -2723.31463 \\
RI-PBE/TZVPP & -2724.8931 \\
B3-LYP/TZVP,SV(P) & -2724.436876 \\
ZPE (kJ/mol) & 734.9 \\
In(qtrans) & 19.64 \\
In(qrot) & 16.11 \\
In(qvib) & 15.33 \\
chem.pot. (kJ/mol) & 608.24 \\
energy $(\mathrm{kJ} / \mathrm{mol})$ & 791.15 \\
entropy $(\mathrm{kJ} / \mathrm{mol} / \mathrm{K})$ & 0.62181 \\
CV $(\mathrm{kJ} / \mathrm{mol}-\mathrm{K})$ & 0.3683726 \\
Cp $((\mathrm{kJ} / \mathrm{mol}-\mathrm{K})$ & 0.3766869 \\
$\mathrm{RI}-\mathrm{PBE} / \mathrm{TZVP}, \mathrm{SV}(\mathrm{P}) \quad\left\langle\mathrm{S}^{2}\right\rangle$ & 3.77 \\
$\left.\mathrm{RI}-\mathrm{PBE} / \mathrm{TZVPP}<\mathrm{S}^{2}\right\rangle$ & 3.77 \\
$\mathrm{~B} 3-\mathrm{LYP} / \mathrm{TZVP}, \mathrm{SV}(\mathrm{P}) \quad\left\langle\mathrm{S}^{2}\right\rangle$ & 3.79 \\
\hline
\end{tabular}

${ }^{1}$ Freeh output; $298.15 \mathrm{~K}, 0.1 \mathrm{MPa}$, unscaled 


\section{Hexachlorobenezene (D6h)}

$$
\begin{aligned}
& \text { RI-PBE/TZVP, SV (P) Cartesian coordinates } \\
& 12 \\
& \text { Energy }=-2987.393876610 \\
& \begin{array}{lrrr}
\mathrm{C} & -1.2243858 & 0.7068995 & 0.0000000 \\
\mathrm{C} & -1.2243858 & -0.7068995 & 0.0000000
\end{array} \\
& \text { C } \quad 0.0000000-1.4137990 \\
& \text { C } \quad 1.2243858 \quad-0.7068995 \quad 0.0000000 \\
& \begin{array}{llll}
\mathrm{C} & 1.2243858 & 0.7068995 & 0.0000000
\end{array} \\
& \begin{array}{llll}
\text { C } & 0.0000000 & 1.4137990 & 0.0000000
\end{array} \\
& \begin{array}{llll}
\mathrm{Cl} & 2.7192072 & -1.5699350 & 0.0000000
\end{array} \\
& \begin{array}{llll}
\mathrm{Cl} & 2.7192072 & 1.5699350 & 0.0000000
\end{array} \\
& \text { Cl } \quad 0.0000000 \quad 3.1398700 \quad 0.0000000 \\
& \begin{array}{llll}
\text { C1 } & -2.7192072 & 1.5699350 & 0.0000000
\end{array} \\
& \text { C1 }-2.7192072 \quad-1.5699350 \quad 0.0000000 \\
& \begin{array}{llll}
\mathrm{Cl} & 0.0000000 & -3.1398700 & 0.0000000
\end{array}
\end{aligned}
$$

Vibrational frequencies

$\begin{array}{rrrrrr}0.00 & 0.00 & 0.00 & 0.00 & 0.00 & 0.00 \\ 66.89 & 66.89 & 92.92 & 156.43 & 209.02 & 209.02 \\ 209.47 & 209.47 & 223.25 & 314.49 & 314.49 & 322.09 \\ 322.09 & 366.87 & 385.16 & 561.98 & 561.98 & 583.01 \\ 606.69 & 692.90 & 692.90 & 873.85 & 873.85 & 1061.91 \\ 1210.39 & 1312.81 & 1331.91 & 1331.91 & 1517.11 & 1517.11\end{array}$

TURBOMOLE Energies and Thermochemistry ${ }^{1}$

\begin{tabular}{lr}
\hline RI-PBE/TZVP,SV(P) & -2987.393877 \\
\hline ZPE & 0.0414601 \\
RI-PBE/TZVP,SV(P) + ZPE & -2987.352417 \\
RI-PBE/TZVPP & -2988.592486 \\
B3-LYP/TZVP,SV(P) & -2988.320408 \\
ZPE (kJ/mol) & 108.9 \\
In(qtrans) & 19.08 \\
In(qrot) & 12.74 \\
In(qvib) & 8.15 \\
chem.pot. $(\mathrm{kJ} / \mathrm{mol})$ & 9.75 \\
energy $(\mathrm{kJ} / \mathrm{mol})$ & 140.11 \\
entropy $(\mathrm{kJ} / \mathrm{mol} / \mathrm{K})$ & 0.44554 \\
CV $(\mathrm{kJ} / \mathrm{mol}-\mathrm{K})$ & 0.1698411 \\
Cp $((\mathrm{kJ} / \mathrm{mol}-\mathrm{K})$ & 0.1781554 \\
\hline
\end{tabular}

${ }^{1}$ Freeh output; $298.15 \mathrm{~K}, 0.1 \mathrm{MPa}$, unscaled 
${ }^{2} \mathbf{A}$

\section{Hexachlorobenzene: $[($ Porphyrin $)(\mathrm{Fe}=\mathbf{O})(\mathrm{SH})$ Prereactant Complex}

RI - PBE/TZVP, SV (P) Cartesian coordinates

52

$\begin{array}{lrrr}\text { Energy }=-5711.003852134 & \\ \mathrm{C} & -3.1701481 & 0.3229686 & 0.2025399 \\ \mathrm{~N} & -2.2308494 & -0.5099264 & 0.7708212 \\ \mathrm{C} & -2.6691066 & -1.7917210 & 0.5455790 \\ \mathrm{C} & -3.9324100 & -1.7772296 & -0.1620613 \\ \mathrm{C} & -4.2415155 & -0.4593655 & -0.3801755 \\ \mathrm{Fe} & -0.4672325 & 0.0481945 & 1.6297895 \\ \mathrm{O} & 0.2181533 & 0.2341511 & 0.1538259 \\ \mathrm{C} & -1.9763808 & -2.9478288 & 0.9121586 \\ \mathrm{C} & -0.7378295 & -2.9685990 & 1.5541790 \\ \mathrm{~N} & -0.0094596 & -1.8620468 & 1.9410317 \\ \mathrm{C} & 1.1239548 & -2.3510608 & 2.5568134 \\ \mathrm{C} & 1.1168549 & -3.7978696 & 2.5491683 \\ \mathrm{C} & -0.0418141 & -4.1821307 & 1.9248474 \\ \mathrm{C} & 2.1342495 & -1.5771179 & 3.1275517 \\ \mathrm{C} & 2.1505873 & -0.1810249 & 3.1576756 \\ \mathrm{~N} & 1.1771448 & 0.6347021 & 2.6284544 \\ \mathrm{C} & 1.6069490 & 1.9283035 & 2.8418192 \\ \mathrm{C} & 2.8750914 & 1.9250340 & 3.5410566 \\ \mathrm{C} & 3.2136658 & 0.6105500 & 3.7389733 \\ \mathrm{C} & 0.9463704 & 3.0930218 & 2.4407638 \\ \mathrm{C} & -0.2679904 & 3.1365726 & 1.7536239 \\ \mathrm{~N} & -1.0102166 & 2.0347372 & 1.4004469 \\ \mathrm{C} & -2.1060897 & 2.5074900 & 0.7146072 \\ \mathrm{C} & -2.0667306 & 3.9550979 & 0.6521147 \\ \mathrm{C} & -0.9245958 & 4.3477236 & 1.3027526 \\ \mathrm{C} & -3.1158630 & 1.7181243 & 0.1622757 \\ \mathrm{~S} & -1.8036320 & 0.2684865 & 3.5367812 \\ \mathrm{H} & -1.0058602 & 1.1337996 & 4.2432911 \\ \mathrm{H} & -0.5421365 & 5.3641293 & 1.4659763 \\ \mathrm{H} & -2.8285027 & 4.5796596 & 0.1667168 \\ \mathrm{H} & -3.9373653 & 2.2376916 & -0.3541122 \\ \mathrm{H} & -5.1132189 & -0.0343097 & -0.8952761 \\ \mathrm{H} & -4.4928692 & -2.6734711 & -0.4591183 \\ \mathrm{H} & -2.4353771 & -3.9170094 & 0.6652255 \\ \mathrm{H} & -0.4126607 & -5.1964749 & 1.7266771 \\ \mathrm{H} & 1.9086681 & -4.4266939 & 2.9774838 \\ \mathrm{H} & 2.9825105 & -2.1063591 & 3.5878153 \\ \mathrm{H} & 4.1052396 & 0.1958914 & 4.2279057 \\ \mathrm{H} & 3.4282579 & 2.8281727 & 3.8314979 \\ \mathrm{H} & 1.4329281 & 4.0526636 & 2.6732086 \\ \mathrm{C} & 2.1381897 & -0.4548031 & -2.0997829 \\ \mathrm{C} & 1.8068477 & 0.8793543 & -2.4215655 \\ \mathrm{C} & 1.1710370 & -1.4753308 & -2.2339693 \\ \mathrm{C} & -0.1237485 & -1.1626819 & -2.7026438 \\ \mathrm{C} & 0.5059903 & 1.1946986 & -2.8704340 \\ \mathrm{C} & -0.4578190 & 0.1729256 & -3.0147234 \\ \mathrm{Cl} & 2.9826474 & 2.1296391 & -2.2351308 \\ \mathrm{Cl} & 0.0862331 & 2.8330988 & -3.2205841 \\ \mathrm{Cl} & -1.3036649 & -2.4128602 & -2.8787308 \\ \mathrm{Cl} & 1.5639231 & -3.1005815 & -1.8014181 \\ \mathrm{Cl} & -2.0532547 & 0.5607635 & -3.5548794 \\ \mathrm{Cl} & 3.7168167 & -0.8352585 & -1.5143875 \\ & & & \end{array}$

Vibrational frequencies

$\begin{array}{rrrrrr}0.00 & 0.00 & 0.00 & 0.00 & 0.00 & 0.00 \\ 4.59 & 7.34 & 14.69 & 19.14 & 21.66 & 37.32 \\ 44.44 & 55.54 & 69.31 & 72.38 & 93.58 & 96.15 \\ 98.96 & 104.23 & 136.05 & 146.29 & 152.17 & 164.89 \\ 181.27 & 205.75 & 208.66 & 208.95 & 209.47 & 209.62 \\ 214.61 & 223.42 & 234.97 & 239.01 & 271.73 & 274.83 \\ 275.35 & 281.03 & 286.98 & 299.79 & 311.98 & 314.13 \\ 314.24 & 328.22 & 329.04 & 345.31 & 358.89 & 359.78 \\ 365.30 & 366.40 & 385.04 & 396.22 & 401.89 & 417.74 \\ 418.97 & 420.24 & 421.99 & 459.05 & 559.86 & 560.42 \\ 591.03 & 605.67 & 607.26 & 649.88 & 656.53 & 656.88 \\ 672.79 & 679.33 & 690.38 & 690.63 & 693.99 & 694.86 \\ 697.63 & 715.71 & 723.93 & 734.18 & 735.20 & 763.69 \\ 771.68 & 772.06 & 778.10 & 781.83 & 784.59 & 784.78 \\ 798.51 & 840.67 & 850.04 & 850.34 & 854.11 & 858.90 \\ 871.67 & 871.74 & 886.79 & 892.08 & 892.36 & 894.45 \\ 993.23 & 1002.05 & 1002.77 & 1011.36 & 1015.08 & 1032.29 \\ 1032.57 & 1036.35 & 1041.23 & 1042.15 & 1042.86 & 1054.80 \\ 1063.82 & 1139.98 & 1148.11 & 1148.67 & 1182.66 & 1183.58 \\ 1210.46 & 1261.45 & 1262.61 & 1314.73 & 1320.56 & 1329.90 \\ 1330.98 & 1338.29 & 1338.79 & 1341.21 & 1364.03 & 1376.24 \\ 1383.00 & 1391.49 & 1392.65 & 1469.64 & 1472.43 & 1473.06 \\ 1504.98 & 1523.31 & 1524.26 & 1525.76 & 1558.13 & 1558.51 \\ 1579.53 & 1602.90 & 1608.97 & 1610.76 & 1662.10 & 2545.27 \\ 3123.89 & 3124.53 & 3125.10 & 3127.51 & 3155.28 & 3155.50 \\ 3156.33 & 3156.51 & 3174.19 & 3174.45 & 3175.30 & 3175.56\end{array}$

TURBOMOLE Energies and Thermochemistry ${ }^{1}$

\begin{tabular}{lr}
\hline RI-PBE/TZVP,SV(P) & -5711.003852 \\
\hline ZPE & 0.3222852 \\
RI-PBE/TZVP,SV(P) + ZPE & -5710.681567 \\
RI-PBE/TZVPP & -5713.495674 \\
B3-LYP/TZVP,SV(P) & -5712.762812 \\
ZPE (kJ/mol) & 846.2 \\
In(qtrans) & 20.43 \\
In(qrot) & 17.5 \\
In(qvib) & 39.42 \\
chem.pot. $(\mathrm{kJ} / \mathrm{mol})$ & 654.4 \\
energy $(\mathrm{kJ} / \mathrm{mol})$ & 940.05 \\
entropy $(\mathrm{kJ} / \mathrm{mol} / \mathrm{K})$ & 0.96637 \\
Cv $(\mathrm{kJ} / \mathrm{mol}-\mathrm{K})$ & 0.5619472 \\
Cp $((\mathrm{kJ} / \mathrm{mol}-\mathrm{K})$ & 0.5702615 \\
$\left.\mathrm{RI}-\mathrm{PBE} / \mathrm{TZVP}, \mathrm{SV}(\mathrm{P}) \quad \mathrm{SS}^{2}\right\rangle$ & 1.10 \\
$\left.\mathrm{RI}-\mathrm{PBE} / \mathrm{TZVPP}<\mathrm{S}^{2}\right\rangle$ & 0.91 \\
$\left.\mathrm{~B} 3-\mathrm{LYP} / \mathrm{TZVP}, \mathrm{SV}(\mathrm{P}) \quad \mathrm{S}^{2}\right\rangle$ & 1.62 \\
\hline
\end{tabular}

${ }^{1}$ Freeh output; $298.15 \mathrm{~K}, 0.1 \mathrm{MPa}$, unscaled 
${ }^{2} \mathbf{A}$

\section{Hexachlorobenzene:[(Porphyrin $)(\mathrm{Fe}=\mathbf{O})(\mathrm{SH})$ Transition State}

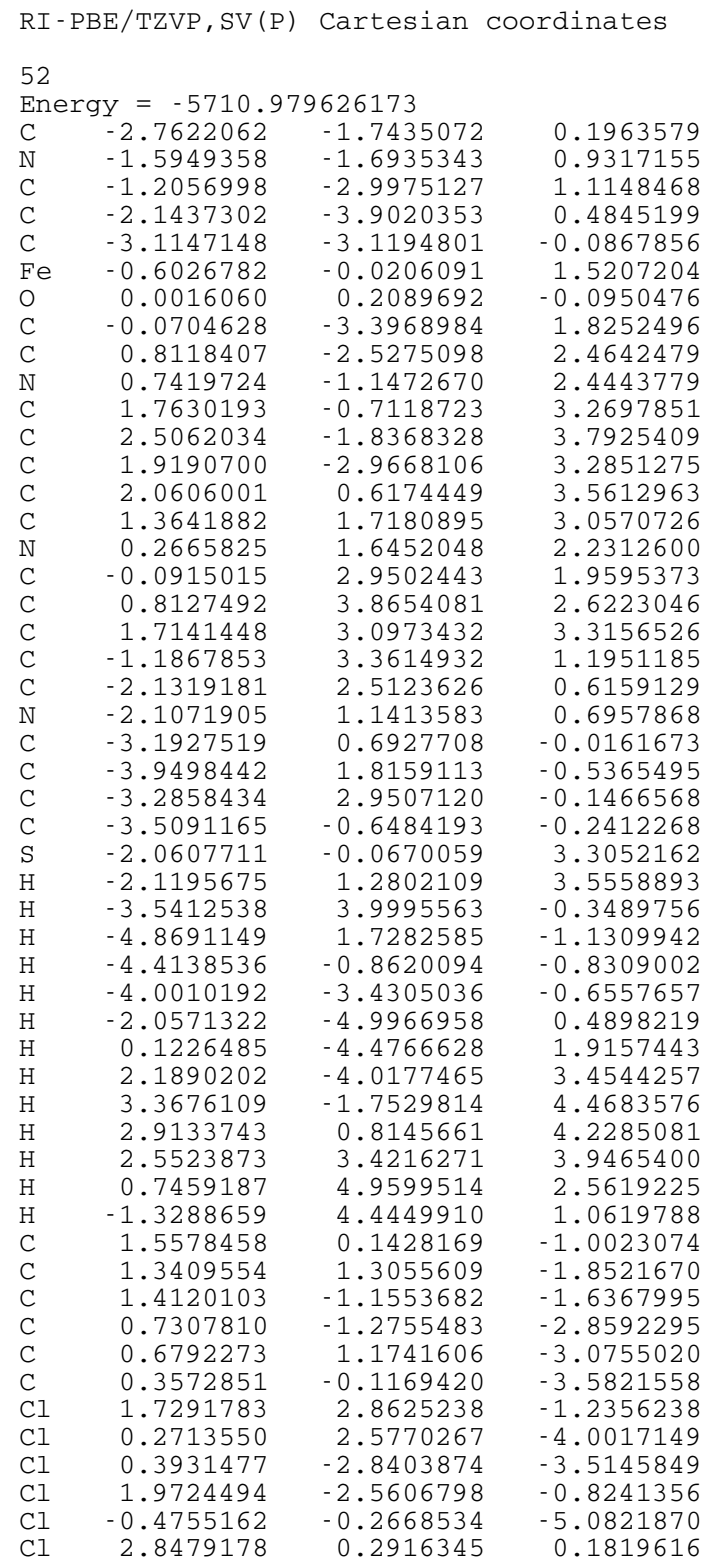

Vibrational frequencies

\begin{tabular}{rrrrrr} 
i542.96 & 0.00 & 0.00 & \multicolumn{1}{c}{0.00} & 0.00 & 0.00 \\
0.00 & 8.32 & 23.16 & 32.04 & 43.46 & 46.57 \\
53.76 & 57.55 & 69.59 & 74.98 & 86.15 & 101.51 \\
103.01 & 105.85 & 117.70 & 144.26 & 149.51 & 166.52 \\
191.27 & 197.38 & 207.10 & 209.37 & 209.81 & 212.78 \\
216.00 & 223.95 & 233.97 & 251.05 & 255.21 & 262.40 \\
272.43 & 277.14 & 285.15 & 297.45 & 302.29 & 307.05 \\
317.15 & 341.04 & 346.22 & 349.55 & 356.39 & 359.34 \\
364.56 & 378.72 & 388.73 & 394.72 & 409.96 & 417.42 \\
420.05 & 421.69 & 422.42 & 458.44 & 485.06 & 566.40 \\
599.20 & 611.58 & 638.82 & 652.36 & 656.56 & 657.78 \\
667.76 & 672.94 & 679.11 & 692.89 & 693.49 & 694.94 \\
698.90 & 702.17 & 716.56 & 724.01 & 734.57 & 735.39 \\
764.96 & 771.90 & 772.86 & 778.40 & 782.32 & 785.00 \\
785.37 & 798.77 & 841.75 & 850.96 & 851.53 & 856.01 \\
859.70 & 867.65 & 886.51 & 891.60 & 892.62 & 894.68 \\
991.72 & 1000.62 & 1002.07 & 1010.69 & 1014.01 & 1031.10 \\
1032.69 & 1036.16 & 1040.58 & 1041.63 & 1042.35 & 1053.42 \\
1060.49 & 1138.91 & 1142.67 & 1147.81 & 1149.00 & 1182.80 \\
1183.57 & 1261.34 & 1263.21 & 1269.58 & 1278.66 & 1317.10 \\
1328.78 & 1332.42 & 1342.31 & 1349.35 & 1363.52 & 1373.90 \\
1382.33 & 1389.89 & 1392.07 & 1440.95 & 1469.70 & 1471.04 \\
1473.41 & 1503.24 & 1505.05 & 1524.60 & 1556.73 & 1559.06 \\
1579.59 & 1604.14 & 1611.83 & 1613.59 & 1662.49 & 2550.04 \\
3122.33 & 3122.88 & 3125.08 & 3125.42 & 3154.18 & 3154.76 \\
3154.92 & 3155.50 & 3173.27 & 3173.81 & 3174.08 & 3174.50
\end{tabular}

TURBOMOLE Energies and Thermochemistry ${ }^{1}$

\begin{tabular}{|c|c|}
\hline RI-PBE/TZVP,SV(P) & -5710.979626 \\
\hline $\mathrm{ZPE}$ & 0.3213085 \\
\hline RI-PBE/TZVP,SV(P) + ZPE & -5710.658318 \\
\hline RI-PBE/TZVPP & -5713.468377 \\
\hline B3-LYP/TZVP,SV(P) & -5712.730375 \\
\hline $\mathrm{ZPE}(\mathrm{kJ} / \mathrm{mol})$ & 843.6 \\
\hline $\ln ($ qtrans $)$ & 20.43 \\
\hline $\ln ($ qrot) & 17.47 \\
\hline $\ln (q v i b)$ & 33.61 \\
\hline chem.pot. (kJ/mol) & 666.31 \\
\hline energy $(\mathrm{kJ} / \mathrm{mol})$ & 934.95 \\
\hline entropy $(\mathrm{kJ} / \mathrm{mol} / \mathrm{K})$ & 0.90932 \\
\hline $\mathrm{Cv}(\mathrm{kJ} / \mathrm{mol}-\mathrm{K})$ & 0.5555372 \\
\hline $\mathrm{Cp}((\mathrm{kJ} / \mathrm{mol}-\mathrm{K})$ & 0.5638515 \\
\hline $\mathrm{RI}-\mathrm{PBE} / \mathrm{TZVP}, \mathrm{SV}(\mathrm{P}) \quad<\mathrm{S}^{2}>$ & 0.94 \\
\hline $\mathrm{RI}-\mathrm{PBE} / \mathrm{TZVPP}<\mathrm{S}^{2}>$ & 0.93 \\
\hline B3 - LYP/TZVP, SV (P) $\quad<\mathrm{S}^{2}>$ & 1.51 \\
\hline
\end{tabular}

${ }^{1}$ Freeh output; $298.15 \mathrm{~K}, 0.1 \mathrm{MPa}$, unscaled 
${ }^{2} \mathbf{A}$

\section{Hexachlorobenzene:[(Porphyrin $)(\mathrm{Fe}=\mathbf{O})(\mathrm{SH})$ Product Complex}

RI-PBE/TZVP, SV (P) Cartesian coordinates

$$
52
$$

Energy $=-5711.039764137$
$\mathrm{C}-3.0204053-0.61656$

\begin{tabular}{lrrr} 
Energy & \multicolumn{3}{l}{-5711.039764137} \\
$\mathrm{C}$ & -3.0204053 & -0.6165674 & 1.6425091 \\
$\mathrm{~N}$ & -1.7380814 & -1.1067512 & 1.7691079 \\
$\mathrm{C}$ & -1.8529543 & -2.4794147 & 1.7235088 \\
$\mathrm{C}$ & -3.2404422 & -2.8657171 & 1.5712410 \\
$\mathrm{C}$ & -3.9670755 & -1.7044230 & 1.5074687 \\
$\mathrm{Fe}$ & -0.0325308 & -0.0552558 & 2.0017263 \\
$\mathrm{O}$ & 0.0730384 & 0.1587842 & -0.0097816 \\
$\mathrm{C}$ & -0.7896131 & -3.3828396 & 1.7529396 \\
$\mathrm{C}$ & 0.5598018 & -3.0297840 & 1.7942971 \\
$\mathrm{~N}$ & 1.0521036 & -1.7430735 & 1.8653260 \\
$\mathrm{C}$ & 2.4249466 & -1.8634422 & 1.8653577 \\
$\mathrm{C}$ & 2.8110461 & -3.2551013 & 1.7744051 \\
$\mathrm{C}$ & 1.6481633 & -3.9823903 & 1.7401074 \\
$\mathrm{C}$ & 3.3271589 & -0.8045575 & 1.9813213 \\
$\mathrm{C}$ & 2.9597489 & 0.5286993 & 2.1674616 \\
$\mathrm{~N}$ & 1.6619940 & 0.9998408 & 2.2093196 \\
$\mathrm{C}$ & 1.7621832 & 2.3522591 & 2.4676785 \\
$\mathrm{C}$ & 3.1510377 & 2.7418240 & 2.5876167 \\
$\mathrm{C}$ & 3.8979092 & 1.6083951 & 2.3889541 \\
$\mathrm{C}$ & 0.6921574 & 3.2432393 & 2.5600979 \\
$\mathrm{C}$ & -0.6467441 & 2.9081235 & 2.3524694 \\
$\mathrm{~N}$ & -1.1192068 & 1.6451988 & 2.0676872 \\
$\mathrm{C}$ & -2.4806676 & 1.7805292 & 1.9007144 \\
$\mathrm{C}$ & -2.8746420 & 3.1643917 & 2.0589642 \\
$\mathrm{C}$ & -1.7328736 & 3.8650842 & 2.3551033 \\
$\mathrm{C}$ & -3.3749194 & 0.7328909 & 1.6738706 \\
$\mathrm{~S}$ & -0.2325713 & -0.3032687 & 4.1638633 \\
$\mathrm{H}$ & 1.1008398 & -0.3540790 & 4.4949354 \\
$\mathrm{H}$ & -1.6186039 & 4.9393105 & 2.5538517 \\
$\mathrm{H}$ & -3.9046882 & 3.5340836 & 1.9680107 \\
$\mathrm{H}$ & -4.4403118 & 0.9839702 & 1.5591421 \\
$\mathrm{H}$ & -5.0512054 & -1.5768252 & 1.3871271 \\
$\mathrm{H}$ & -3.5956658 & -3.9031786 & 1.5086612 \\
$\mathrm{H}$ & -1.0317780 & -4.4551495 & 1.7010323 \\
$\mathrm{H}$ & 1.5205734 & -5.0713895 & 1.6775063 \\
$\mathrm{H}$ & 3.8484877 & -3.6145152 & 1.7501793 \\
$\mathrm{H}$ & 4.4015843 & -1.0423745 & 1.9694436 \\
$\mathrm{H}$ & 4.9898084 & 1.4945462 & 2.3957504 \\
$\mathrm{H}$ & 3.4955888 & 3.7646407 & 2.7888661 \\
$\mathrm{H}$ & 0.9256137 & 4.2969488 & 2.7776986 \\
$\mathrm{C}$ & 0.0664950 & 0.2359274 & -1.2470297 \\
$\mathrm{C}$ & 0.0748089 & 1.4994733 & -1.9627474 \\
$\mathrm{C}$ & 0.0391751 & -1.0666547 & -2.0894856 \\
$\mathrm{C}$ & 0.0870296 & -0.8969036 & -3.5804724 \\
$\mathrm{C}$ & 0.0979902 & 1.5565283 & -3.3505444 \\
$\mathrm{C}$ & 0.1093306 & 0.3455958 & -4.1636566 \\
$\mathrm{Cl}$ & 0.0665263 & 2.9334850 & -1.0089368 \\
$\mathrm{Cl}$ & 0.1182109 & 3.0795239 & -4.1505980 \\
$\mathrm{Cl}$ & 0.0862425 & -2.3375809 & -4.5215714 \\
$\mathrm{Cl}$ & -1.5145923 & -1.9178979 & -1.6668482 \\
$\mathrm{Cl}$ & 0.1481474 & 0.5045334 & -5.8836855 \\
$\mathrm{Cl}$ & 1.4543910 & -2.0713571 & -1.5721377 \\
& & & \\
\hline
\end{tabular}

Vibrational frequencies

$\begin{array}{rrrrrr}0.00 & 0.00 & 0.00 & 0.00 & 0.00 & 0.00 \\ 5.90 & 20.52 & 22.48 & 33.81 & 50.31 & 51.00 \\ 53.24 & 55.76 & 69.91 & 71.62 & 92.04 & 103.56 \\ 112.97 & 117.77 & 146.88 & 147.67 & 150.91 & 168.40 \\ 171.44 & 196.65 & 208.88 & 211.21 & 220.42 & 222.03 \\ 225.13 & 232.28 & 257.69 & 261.12 & 262.79 & 274.22 \\ 280.13 & 283.76 & 301.15 & 304.47 & 313.20 & 319.63 \\ 325.94 & 341.43 & 359.20 & 359.84 & 359.90 & 366.78 \\ 369.97 & 386.35 & 400.89 & 412.62 & 420.14 & 420.90 \\ 422.04 & 422.46 & 460.30 & 476.58 & 495.95 & 577.21 \\ 629.72 & 635.60 & 652.24 & 657.10 & 657.97 & 672.67 \\ 673.64 & 685.26 & 695.54 & 696.51 & 697.39 & 702.42 \\ 720.32 & 722.85 & 730.73 & 734.98 & 736.16 & 745.83 \\ 761.41 & 769.02 & 770.22 & 776.88 & 784.64 & 785.51 \\ 786.30 & 799.48 & 834.45 & 834.64 & 842.88 & 843.22 \\ 850.49 & 884.84 & 889.47 & 890.50 & 892.37 & 933.03 \\ 992.13 & 999.79 & 1003.64 & 1009.69 & 1011.68 & 1013.83 \\ 1030.49 & 1031.59 & 1035.09 & 1040.46 & 1041.83 & 1042.52 \\ 1054.59 & 1138.59 & 1144.01 & 1144.50 & 1147.09 & 1181.22 \\ 1183.12 & 1185.04 & 1260.88 & 1261.92 & 1272.52 & 1328.93 \\ 1331.49 & 1333.60 & 1345.02 & 1365.61 & 1376.22 & 1387.11 \\ 1389.10 & 1390.55 & 1467.66 & 1471.03 & 1472.84 & 1474.49 \\ 1507.43 & 1521.99 & 1554.17 & 1557.48 & 1559.52 & 1579.90 \\ 1603.50 & 1609.55 & 1613.01 & 1621.23 & 1659.08 & 2516.99 \\ 3119.58 & 3124.07 & 3124.23 & 3124.71 & 3152.69 & 3152.83 \\ 3153.53 & 3156.38 & 3172.10 & 3172.32 & 3172.79 & 3175.67\end{array}$

TURBOMOLE Energies and Thermochemistry ${ }^{1}$

\begin{tabular}{lr}
\hline RI-PBE/TZVP,SV(P) & -5711.039764 \\
\hline ZPE & 0.3231361 \\
RI-PBE/TZVP,SV(P) + ZPE & -5710.716628 \\
RI-PBE/TZVPP & -5713.524326 \\
B3-LYP/TZVP,SV(P) & \\
ZPE (kJ/mol) & 848.4 \\
In(qtrans) & 20.43 \\
In(qrot) & 17.6 \\
In(qvib) & 35.33 \\
chem.pot. $(\mathrm{kJ} / \mathrm{mol})$ & 666.54 \\
energy $(\mathrm{kJ} / \mathrm{mol})$ & 940.62 \\
entropy $(\mathrm{kJ} / \mathrm{mol} / \mathrm{K})$ & 0.92759 \\
CV $(\mathrm{kJ} / \mathrm{mol}-\mathrm{K})$ & 0.5592496 \\
Cp $((\mathrm{kJ} / \mathrm{mol}-\mathrm{K})$ & 0.5675639 \\
$\mathrm{RI}-\mathrm{PBE} / \mathrm{TZVP}, \mathrm{SV}(\mathrm{P}) \quad \mathrm{CS}^{2}>$ & 0.91 \\
$\mathrm{RI}-\mathrm{PBE} / \mathrm{TZVPP} \quad\left\langle\mathrm{S}^{2}\right\rangle$ & 0.93 \\
$\mathrm{~B} 3-\mathrm{LYP} / \mathrm{TZVP}, \mathrm{SV}(\mathrm{P}) \quad\left\langle\mathrm{S}^{2}\right\rangle$ & 122.8 \\
\hline
\end{tabular}

${ }^{1}$ Freeh output; $298.15 \mathrm{~K}, 0.1 \mathrm{MPa}$, unscaled 


\section{2,2,3,4,5,6-Hexachloro-3,5-cyclohexadieneone}

$$
13
$$

TURBOMOLE Energies and Thermochemistry ${ }^{1}$

\begin{tabular}{lr}
\hline RI-PBE/TZVP,SV(P) & -3062.45303 \\
\hline ZPE & 0.0448018 \\
RI-PBE/TZVP,SV(P) + ZPE & -3062.408228 \\
RI-PBE/TZVPP & -3063.740388 \\
B3-LYP/TZVP,SV(P) & -3063.415436 \\
ZPE (kJ/mol) & 117.6 \\
In(qtrans) & 19.16 \\
In(qrot) & 15.27 \\
In(qvib) & 10.68 \\
chem.pot. (kJ/mol) & 5.8 \\
energy (kJ/mol) & 152.22 \\
entropy $(\mathrm{kJ} / \mathrm{mol} / \mathrm{K})$ & 0.49941 \\
CV $(\mathrm{kJ} / \mathrm{mol}-\mathrm{K})$ & 0.18648 \\
Cp $((\mathrm{kJ} / \mathrm{mol}-\mathrm{K})$ & 0.1947943 \\
\hline
\end{tabular}

${ }^{1}$ Freeh output; $298.15 \mathrm{~K}, 0.1 \mathrm{MPa}$, unscaled 


\section{${ }^{2}$ A [(Porphyrin)(Fe)(SH)}

\section{RI - PBE/TZVP, SV(P) Cartesian coordinates}

39

Energy $=-2648.570389303$

$\begin{array}{lrrr}\mathrm{C} & -1.5714796 & -2.5999041 & -0.1067125 \\ \mathrm{~N} & -0.3430191 & -1.9593683 & -0.1520483 \\ \mathrm{C} & 0.5859538 & -2.9883374 & -0.1847460 \\ \mathrm{C} & -0.0643052 & -4.2816188 & -0.1411833 \\ \mathrm{C} & -1.4102769 & -4.0390081 & -0.0876932 \\ \mathrm{Fe} & 0.0013318 & 0.0042198 & -0.0300728 \\ \mathrm{C} & 1.9617446 & -2.8159664 & -0.2837914 \\ \mathrm{C} & 2.5952476 & -1.5757244 & -0.3314472 \\ \mathrm{~N} & 1.9534769 & -0.3470140 & -0.2867290 \\ \mathrm{C} & 2.9796005 & 0.5854896 & -0.2522149 \\ \mathrm{C} & 4.2673199 & -0.0594542 & -0.3253625 \\ \mathrm{C} & 4.0271477 & -1.4102381 & -0.3791486 \\ \mathrm{C} & 2.8100625 & 1.9642393 & -0.1426245 \\ \mathrm{C} & 1.5754702 & 2.6020587 & -0.0989424 \\ \mathrm{~N} & 0.3461584 & 1.9600129 & -0.1461201 \\ \mathrm{C} & -0.5830428 & 2.9910350 & -0.1793458 \\ \mathrm{C} & 0.0675882 & 4.283499 & -0.1348358 \\ \mathrm{C} & 1.4138329 & 4.0404521 & -0.0800885 \\ \mathrm{C} & -1.9593359 & 2.8213359 & -0.2821827 \\ \mathrm{C} & -2.5937453 & 1.5819613 & -0.3334481 \\ \mathrm{~N} & -1.9495946 & 0.3541738 & -0.2883088 \\ \mathrm{C} & -2.9740833 & -0.5806083 & -0.2568199 \\ \mathrm{C} & -4.2626400 & 0.0629341 & -0.3316648 \\ \mathrm{C} & -4.0251146 & 1.4143471 & -0.3843850 \\ \mathrm{C} & -2.8047711 & -1.9600888 & -0.1495025 \\ \mathrm{~S} & -0.0185939 & -0.0652898 & 2.1198833 \\ \mathrm{H} & -0.0209921 & 1.2812669 & 2.4196322 \\ \mathrm{H} & -4.7483877 & 2.2387741 & -0.4413965 \\ \mathrm{H} & -5.2243514 & -0.4674718 & -0.3374996 \\ \mathrm{H} & -3.7100619 & -2.5856963 & -0.1217650 \\ \mathrm{H} & -2.2396358 & -4.7583098 & -0.0569573 \\ \mathrm{H} & 0.4632112 & -5.2446080 & -0.1627673 \\ \mathrm{H} & 2.5939785 & -3.7164725 & -0.3137960 \\ \mathrm{H} & 4.7489724 & -2.2359872 & -0.4351886 \\ \mathrm{H} & 5.2300018 & 0.4692078 & -0.3282559 \\ \mathrm{H} & 3.7143768 & 2.5911353 & -0.1133856 \\ \mathrm{H} & 2.2431986 & 4.7598189 & -0.0508404 \\ \mathrm{H} & -0.4594709 & 5.2466986 & -0.1592764 \\ \mathrm{H} & -2.5898553 & 3.7230785 & -0.3133628\end{array}$

Vibrational frequencies

$\begin{array}{rrrrrr}0.00 & 0.00 & 0.00 & 0.00 & 0.00 & 0.00 \\ 9.20 & 41.81 & 75.19 & 89.00 & 98.62 & 112.12 \\ 140.40 & 141.28 & 225.37 & 229.14 & 249.28 & 251.35 \\ 259.30 & 265.50 & 284.34 & 286.05 & 290.23 & 340.15 \\ 359.74 & 361.68 & 361.96 & 404.43 & 408.03 & 415.82 \\ 417.13 & 423.09 & 423.37 & 425.13 & 458.56 & 657.46 \\ 658.47 & 659.16 & 669.96 & 672.39 & 681.41 & 700.02 \\ 700.44 & 704.85 & 718.16 & 724.83 & 735.84 & 736.61 \\ 768.58 & 773.35 & 773.81 & 780.68 & 786.51 & 787.17 \\ 788.81 & 800.58 & 844.64 & 852.84 & 854.42 & 862.60 \\ 886.90 & 891.86 & 893.53 & 895.51 & 987.49 & 989.90 \\ 997.26 & 1003.13 & 1004.12 & 1027.60 & 1029.12 & 1035.41 \\ 1038.43 & 1039.11 & 1040.57 & 1048.32 & 1131.86 & 1141.06 \\ 1142.64 & 1182.08 & 1182.67 & 1260.73 & 1262.15 & 1311.11 \\ 1329.61 & 1338.77 & 1348.01 & 1364.05 & 1369.92 & 1370.88 \\ 1372.97 & 1376.67 & 1472.73 & 1474.32 & 1485.45 & 1512.39 \\ 1525.96 & 1559.02 & 1566.87 & 1585.98 & 1622.66 & 1629.07 \\ 1632.53 & 1670.31 & 2470.80 & 3122.40 & 3122.54 & 3122.64 \\ 3123.27 & 3152.59 & 3153.12 & 3153.34 & 3153.37 & 3172.10 \\ 3172.36 & 3172.43 & 3172.80 & & & \\ & & & & & \end{array}$

TURBOMOLE Energies and Thermochemistry ${ }^{1}$

\begin{tabular}{|c|c|}
\hline RI - PBE/TZVP , SV (P) & -2648.570389 \\
\hline $\mathrm{ZPE}$ & 0.2775612 \\
\hline RI-PBE/TZVP, SV (P) + ZPE & -2648.292828 \\
\hline RI - PBE/TZVPP & -2649.779061 \\
\hline B3 - LYP / TZVP , SV (P) & -2649.392864 \\
\hline $\mathrm{ZPE} \quad(\mathrm{kJ} / \mathrm{mol})$ & 728.7 \\
\hline $\ln ($ qtrans) & 19.58 \\
\hline $\ln ($ grot $)$ & 16.08 \\
\hline $\ln (q v i b)$ & 16.21 \\
\hline chem.pot. (kJ/mol) & 600.15 \\
\hline energy $(\mathrm{kJ} / \mathrm{mol})$ & 781.82 \\
\hline entropy $(\mathrm{kJ} / \mathrm{mol} / \mathrm{K})$ & 0.61764 \\
\hline $\mathrm{CV} \quad(\mathrm{kJ} / \mathrm{mol}-\mathrm{K})$ & 0.3483161 \\
\hline $\mathrm{Cp} \quad((\mathrm{kJ} / \mathrm{mol}-\mathrm{K})$ & 0.3566304 \\
\hline $\mathrm{RI}-\mathrm{PBE} / \mathrm{TZVP}, \mathrm{SV}(\mathrm{P}) \quad\left\langle\mathrm{S}^{2}>\right.$ & 0.77 \\
\hline $\mathrm{RI}-\mathrm{PBE} / \mathrm{TZVPP} \quad<\mathrm{S}^{2}>$ & 0.77 \\
\hline B3 - LYP / TZVP , SV (P) $\quad<\mathrm{S}^{2}>$ & 0.79 \\
\hline
\end{tabular}

${ }^{1}$ Freeh output; $298.15 \mathrm{~K}, 0.1 \mathrm{MPa}$, unscaled 


\section{${ }^{4} \mathrm{~A}[($ Porphyrin $)(\mathrm{Fe}=\mathrm{O})(\mathrm{SH})$}

\section{RI - PBE/TZVP, SV(P) Cartesian coordinates}

\section{0}

Energy $=-2723.594528869$

$\begin{array}{lrrr}\mathrm{C} & 1.3220657 & -2.7087746 & 0.0676101 \\ \mathrm{~N} & 1.4732550 & -1.3502404 & -0.0992487 \\ \mathrm{C} & 2.8152660 & -1.1313986 & -0.3087462 \\ \mathrm{C} & 3.5383431 & -2.3854032 & -0.2648509 \\ \mathrm{C} & 2.6110562 & -3.3651865 & -0.0124268 \\ \mathrm{Fe} & 0.0063679 & 0.0149872 & -0.1987373 \\ \mathrm{O} & 0.0222095 & 0.0018788 & -1.8624260 \\ \mathrm{C} & 3.4045509 & 0.1274509 & -0.4485098 \\ \mathrm{C} & 2.7293850 & 1.3443856 & -0.3063918 \\ \mathrm{~N} & 1.3779970 & 1.4816626 & -0.0957020 \\ \mathrm{C} & 1.1588287 & 2.8176865 & 0.1364731 \\ \mathrm{C} & 2.4084111 & 3.5498408 & 0.0824825 \\ \mathrm{C} & 3.3840850 & 2.6348471 & -0.2224920 \\ \mathrm{C} & -0.0971769 & 3.4085127 & 0.3005575 \\ \mathrm{C} & -1.3151430 & 2.7443969 & 0.1306306 \\ \mathrm{~N} & -1.4557534 & 1.3948280 & -0.0978771 \\ \mathrm{C} & -2.7961430 & 1.1837594 & -0.3290345 \\ \mathrm{C} & -3.5222607 & 2.4352774 & -0.2567632 \\ \mathrm{C} & -2.6028365 & 3.4037181 & 0.0593407 \\ \mathrm{C} & -3.3993587 & -0.0693136 & -0.4800067 \\ \mathrm{C} & -2.7408440 & -1.2903611 & -0.3191488 \\ \mathrm{~N} & -1.3883629 & -1.4268134 & -0.0986432 \\ \mathrm{C} & -1.1574901 & -2.7775240 & 0.0758514 \\ \mathrm{C} & -2.4066044 & -3.5057902 & -0.0000118 \\ \mathrm{C} & -3.3882871 & -2.5844843 & -0.2678384 \\ \mathrm{C} & 0.0993131 & -3.3762316 & 0.1822939 \\ \mathrm{~S} & -0.0015070 & -0.2505369 & 2.2298753 \\ \mathrm{H} & -1.3264945 & 0.0326486 & 2.4459120 \\ \mathrm{H} & -4.4653827 & -2.7506369 & -0.4030856 \\ \mathrm{H} & -2.5012455 & -4.5941749 & 0.1121315 \\ \mathrm{H} & 0.1296625 & -4.4710597 & 0.2920443 \\ \mathrm{H} & 2.7694354 & -4.4461045 & 0.0992641 \\ \mathrm{H} & 4.6237455 & -2.4864271 & -0.3980532 \\ \mathrm{H} & 4.4944281 & 0.1655491 & -0.5981363 \\ \mathrm{H} & 4.4619518 & 2.7990467 & -0.3536637 \\ \mathrm{H} & 2.5062582 & 4.6325895 & 0.2377492 \\ \mathrm{H} & -0.1301534 & 4.4951259 & 0.4732071 \\ \mathrm{H} & -2.7638402 & 4.4794630 & 0.2104346 \\ \mathrm{H} & -4.6058235 & 2.5388594 & -0.4028312 \\ \mathrm{H} & -4.4872588 & -0.0939105 & -0.6457373\end{array}$

Vibrational frequencies

$\begin{array}{rrrrrr}0.00 & 0.00 & 0.00 & 0.00 & 0.00 & 0.00 \\ 45.48 & 52.34 & 87.56 & 90.22 & 101.70 & 139.54 \\ 146.02 & 167.88 & 171.14 & 177.90 & 211.84 & 217.59 \\ 233.49 & 265.70 & 269.81 & 271.19 & 280.03 & 285.89 \\ 312.12 & 313.45 & 343.94 & 358.38 & 359.61 & 368.70 \\ 402.26 & 405.63 & 419.46 & 421.09 & 422.09 & 423.85 \\ 458.53 & 588.05 & 650.51 & 653.43 & 654.09 & 667.89 \\ 674.37 & 692.31 & 693.19 & 697.28 & 714.09 & 724.26 \\ 734.37 & 735.18 & 762.54 & 769.14 & 769.65 & 775.11 \\ 779.00 & 785.51 & 786.24 & 799.95 & 811.47 & 842.41 \\ 851.06 & 851.15 & 859.42 & 885.86 & 891.16 & 891.24 \\ 893.45 & 994.10 & 1003.75 & 1004.70 & 1011.08 & 1016.54 \\ 1031.38 & 1033.32 & 1037.30 & 1041.26 & 1041.83 & 1043.52 \\ 1055.85 & 1137.52 & 1146.72 & 1148.02 & 1181.14 & 1185.55 \\ 1261.62 & 1262.62 & 1297.39 & 1326.90 & 1327.89 & 1339.21 \\ 1363.83 & 1371.39 & 1381.01 & 1385.87 & 1395.76 & 1465.14 \\ 1468.16 & 1469.01 & 1500.93 & 1521.63 & 1553.94 & 1554.03 \\ 1573.65 & 1584.11 & 1602.61 & 1604.08 & 1654.97 & 2551.76 \\ 3124.45 & 3125.35 & 3125.44 & 3125.59 & 3155.49 & 3155.69 \\ 3155.90 & 3156.08 & 3174.29 & 3174.44 & 3174.73 & 3174.90\end{array}$

TURBOMOLE Energies and Thermochemistry ${ }^{1}$

\begin{tabular}{|c|c|}
\hline RI - PBE/TZVP , SV (P) & -2723.594529 \\
\hline $\mathrm{ZPE}$ & 0.2798986 \\
\hline RI-PBE/TZVP, SV (P) + ZPE & -2723.31463 \\
\hline RI - PBE/TZVPP & -2724.8931 \\
\hline B3 - LYP / TZVP , SV (P) & -2724.436876 \\
\hline $\mathrm{ZPE} \quad(\mathrm{kJ} / \mathrm{mol})$ & 734.9 \\
\hline $\ln ($ qtrans) & 19.64 \\
\hline $\ln ($ grot $)$ & 16.11 \\
\hline $\ln ($ qvib) & 15.33 \\
\hline chem.pot. (kJ/mol) & 608.24 \\
\hline energy $(\mathrm{kJ} / \mathrm{mol})$ & 791.15 \\
\hline entropy $(\mathrm{kJ} / \mathrm{mol} / \mathrm{K})$ & 0.62181 \\
\hline $\mathrm{CV} \quad(\mathrm{kJ} / \mathrm{mol}-\mathrm{K})$ & 0.3683726 \\
\hline $\mathrm{Cp} \quad((\mathrm{kJ} / \mathrm{mol}-\mathrm{K})$ & 0.3766869 \\
\hline $\mathrm{RI}-\mathrm{PBE} / \mathrm{TZVP}, \mathrm{SV}(\mathrm{P}) \quad\left\langle\mathrm{S}^{2}>\right.$ & 3.78 \\
\hline $\mathrm{RI}-\mathrm{PBE} / \mathrm{TZVPP} \quad<\mathrm{S}^{2}>$ & 3.78 \\
\hline B3 - LYP / TZVP , SV (P) $\quad<\mathrm{S}^{2}>$ & 3.79 \\
\hline
\end{tabular}

${ }^{1}$ Freeh output; $298.15 \mathrm{~K}, 0.1 \mathrm{MPa}$, unscaled 
${ }^{4} \mathbf{A}$

\section{Hexachlorobenzene:[(Porphyrin $)(\mathrm{Fe}=\mathbf{O})(\mathrm{SH})$ Prereactant Complex}

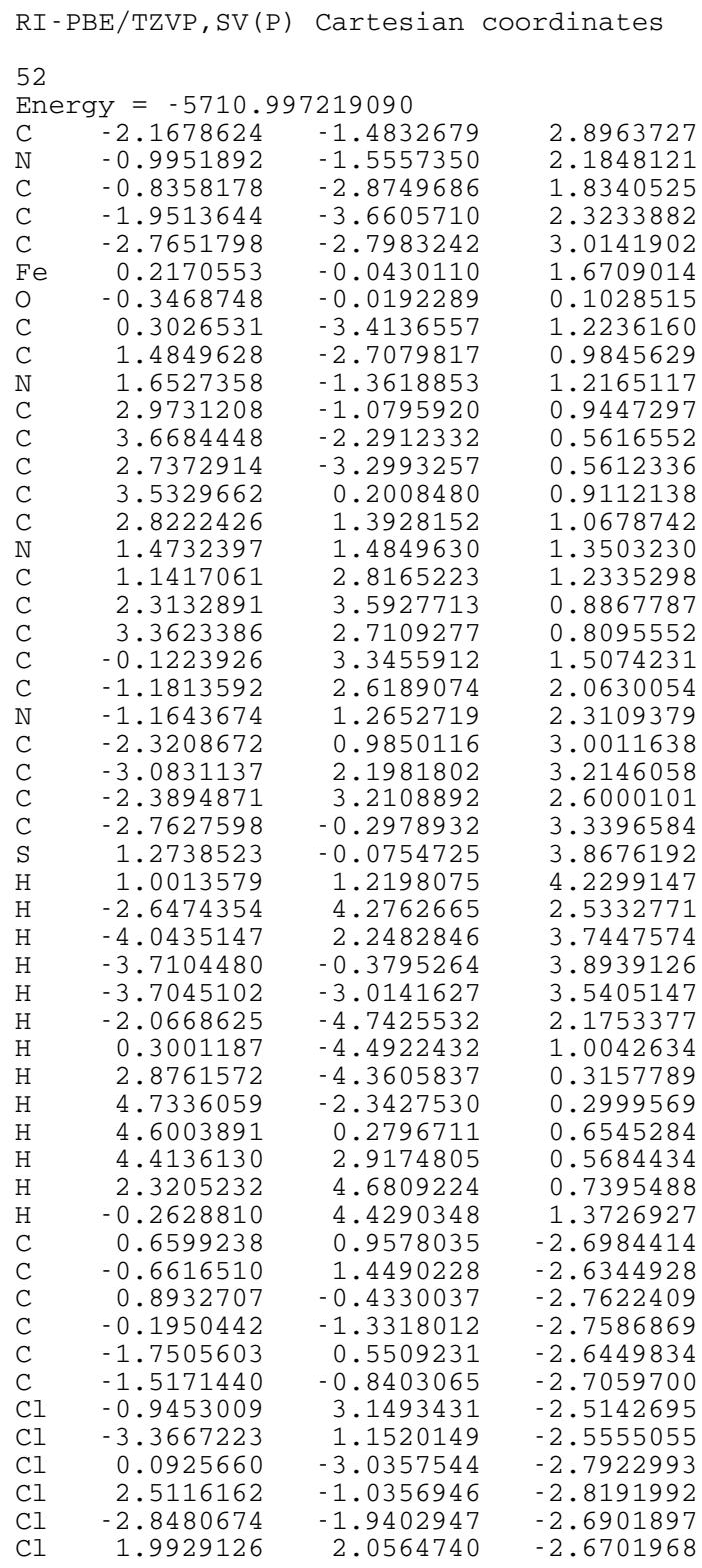

Vibrational frequencies

$\begin{array}{rrrrrr}i 3.24 & 0.00 & 0.00 & 0.00 & 0.00 & 0.00 \\ 0.00 & 14.42 & 16.65 & 21.68 & 22.66 & 44.38 \\ 52.94 & 56.34 & 70.35 & 71.37 & 82.23 & 90.44 \\ 95.80 & 102.76 & 139.60 & 146.38 & 157.47 & 163.64 \\ 172.11 & 176.01 & 209.20 & 209.30 & 209.88 & 209.96 \\ 212.56 & 219.42 & 223.81 & 235.57 & 267.47 & 271.25 \\ 272.56 & 279.98 & 286.03 & 310.72 & 314.25 & 314.28 \\ 317.03 & 326.45 & 327.30 & 343.51 & 359.42 & 360.65 \\ 366.50 & 371.56 & 385.21 & 404.39 & 406.14 & 420.02 \\ 421.21 & 422.45 & 424.68 & 458.08 & 529.43 & 558.80 \\ 559.41 & 596.49 & 606.54 & 649.92 & 652.82 & 653.61 \\ 667.27 & 673.65 & 689.73 & 690.93 & 691.57 & 692.70 \\ 697.01 & 713.82 & 723.86 & 733.74 & 734.77 & 762.25 \\ 768.53 & 769.26 & 774.14 & 778.15 & 785.64 & 786.54 \\ 800.16 & 808.52 & 842.42 & 850.30 & 851.90 & 859.61 \\ 870.76 & 871.78 & 886.16 & 891.21 & 891.80 & 893.98 \\ 995.52 & 1004.86 & 1005.66 & 1010.83 & 1017.70 & 1030.82 \\ 1033.14 & 1036.18 & 1040.40 & 1041.00 & 1043.17 & 1055.82 \\ 1065.32 & 1138.27 & 1146.49 & 1149.30 & 1181.96 & 1184.97 \\ 1211.71 & 1261.50 & 1263.07 & 1293.28 & 1320.38 & 1325.48 \\ 1326.55 & 1338.17 & 1338.68 & 1339.25 & 1362.92 & 1371.99 \\ 1380.70 & 1385.14 & 1395.05 & 1463.46 & 1466.71 & 1467.23 \\ 1499.34 & 1520.45 & 1524.68 & 1526.30 & 1551.97 & 1552.41 \\ 1571.69 & 1579.58 & 1599.69 & 1601.79 & 1652.36 & 2547.68 \\ 3124.66 & 3125.79 & 3126.17 & 3126.49 & 3155.96 & 3156.24 \\ 3156.47 & 3156.95 & 3174.67 & 3175.04 & 3175.14 & 3175.67\end{array}$

TURBOMOLE Energies and Thermochemistry ${ }^{1}$ RI-PBE/TZVP,SV(P)

$-5710.997215$

ZPE 0.3216023

RI-PBE/TZVP,SV(P) + ZPE

RI-PBE/TZVPP

B3-LYP/TZVP,SV(P)

$\mathrm{ZPE}(\mathrm{kJ} / \mathrm{mol})$

$-5710.675617$

In(qtrans)

$-5713.489127$

$-5712.762071$

In(qrot)

844.4

20.43

In(qvib)

17.51

chem.pot. $(\mathrm{kJ} / \mathrm{mol})$

34.68

energy $(\mathrm{kJ} / \mathrm{mol})$

664.35

entropy $(\mathrm{kJ} / \mathrm{mol} / \mathrm{K})$

936.08

Cv $(\mathrm{kJ} / \mathrm{mol}-\mathrm{K})$

0.9197

$\mathrm{Cp}((\mathrm{kJ} / \mathrm{mol}-\mathrm{K})$

0.5551052

$\mathrm{RI}-\mathrm{PBE} / \mathrm{TZVP}, \mathrm{SV}(\mathrm{P}) \quad\left\langle\mathrm{S}^{2}>\right.$

0.5634195

$\mathrm{RI}-\mathrm{PBE} / \mathrm{TZVPP}<\mathrm{S}^{2}>$

3.77

B3 - LYP/TZVP, SV (P) $<\mathrm{S}^{2}>$

3.77

${ }^{1}$ Freeh output; $298.15 \mathrm{~K}, 0.1 \mathrm{MPa}$, unscaled 
${ }^{4} \mathrm{~A}$

\section{Hexachlorobenzene: $[($ Porphyrin $)(\mathbf{F e}=\mathbf{O})(\mathrm{SH})$ Transition State}

RI-PBE/TZVP, SV (P) Cartesian coordinates

52

\begin{tabular}{|c|c|c|c|}
\hline $\mathrm{C}$ & -3.2016668 & -1.1657086 & 0.3659227 \\
\hline $\mathrm{N}$ & -1.9589406 & -1.3935416 & 0.9075169 \\
\hline C & -1.7729546 & -2.7555233 & 0.8731520 \\
\hline C & -2.9251783 & -3.4084377 & 0.2841656 \\
\hline $\mathrm{C}$ & -3.8238348 & -2.4176093 & -0.0172665 \\
\hline $\mathrm{Fe}$ & -0.6345578 & -0.0028517 & 1.5376400 \\
\hline 0 & 0.1090305 & -0.0374409 & -0.0356731 \\
\hline $\mathrm{C}$ & -0.6700803 & -3.4305470 & 1.4018109 \\
\hline $\mathrm{C}$ & 0.3817049 & -2.8239162 & 2.0933171 \\
\hline $\mathrm{N}$ & 0.5283459 & -1.4700893 & 2.2866240 \\
\hline C & 1.6608808 & -1.3144172 & 3.0551444 \\
\hline C & 2.2388910 & -2.6049634 & 3.3645873 \\
\hline $\mathrm{C}$ & 1.4491424 & -3.5456679 & 2.7532400 \\
\hline $\mathrm{C}$ & 2.2195893 & -0.0920953 & 3.4350490 \\
\hline $\mathrm{C}$ & 1.7350197 & 1.1635878 & 3.0652432 \\
\hline $\mathbb{N}$ & 0.6152910 & 1.3919973 & 2.2948101 \\
\hline & 0.5417507 & 2.7552743 & 2.1254337 \\
\hline & 1.6462628 & 3.4079636 & 2.7971167 \\
\hline $\mathrm{C}$ & 2.3821700 & 2.4162968 & 3.3943836 \\
\hline $\mathrm{C}$ & -0.4809167 & 3.4272203 & 1.4518625 \\
\hline C & -1.6256700 & 2.8192194 & 0.9297388 \\
\hline $\mathrm{N}$ & -1.8847289 & 1.4666337 & 0.9482862 \\
\hline C & -3.1392301 & 1.3130837 & 0.4014067 \\
\hline C & -3.6940431 & 2.6015923 & 0.0423232 \\
\hline $\mathrm{C}$ & -2.7432121 & 3.5394670 & 0.3563844 \\
\hline $\mathrm{C}$ & -3.7727593 & 0.0918484 & 0.1578207 \\
\hline S & -1.6216085 & -0.0077267 & 3.6683605 \\
\hline & -1.9236079 & 1.3290005 & 3.7347877 \\
\hline & -2.7840207 & 4.6292584 & 0.2276307 \\
\hline & -4.6835679 & 2.7513244 & -0.4097035 \\
\hline & -4.7785555 & 0.1240212 & -0.2881362 \\
\hline & -4.8197770 & -2.5073039 & -0.4710068 \\
\hline H & -3.0241492 & -4.4927821 & 0.1415746 \\
\hline$H$ & -0.6562403 & -4.5278184 & 1.3166035 \\
\hline H & 1.5585943 & -4.6383827 & 2.7515209 \\
\hline $\mathrm{H}$ & 3.1438050 & -2.7551036 & 3.9682922 \\
\hline $\mathrm{H}$ & 3.1359377 & -0.1219023 & 4.0444732 \\
\hline $\mathrm{H}$ & 3.2923223 & 2.5077774 & 4.0020296 \\
\hline$H$ & 1.8148213 & 4.4930573 & 2.8128013 \\
\hline & -0.4091216 & 4.5232302 & 1.3795414 \\
\hline & 1.6196179 & -0.0130065 & -1.1287052 \\
\hline & 1.3756057 & 1.2279555 & -1.8352450 \\
\hline & 1.3629589 & -1.2396743 & -1.8573143 \\
\hline & 0.6213672 & -1.2194674 & -3.0456380 \\
\hline & 0.6308087 & 1.2360754 & -3.0228790 \\
\hline & 0.2458075 & 0.0158264 & -3.6359784 \\
\hline & 1.9002899 & 2.7091454 & -1.1324994 \\
\hline & 0.2178025 & 2.7370823 & -3.7775260 \\
\hline & 0.1941925 & -2.7024361 & -3.8273017 \\
\hline & 1.8563687 & -2.7374898 & -1.1675111 \\
\hline & -0.6703725 & 0.0327977 & -5.0957345 \\
\hline & 2.9651759 & -0.0309569 & -0.0062356 \\
\hline
\end{tabular}

Vibrational frequencies

$\begin{array}{rrrrrr}1527.18 & 0.00 & 0.00 & 0.00 & 0.00 & 0.00 \\ 0.00 & 7.06 & 21.68 & 30.57 & 37.94 & 46.57 \\ 51.95 & 52.67 & 68.79 & 75.16 & 79.14 & 92.91 \\ 101.10 & 105.11 & 123.05 & 137.85 & 148.22 & 153.97 \\ 193.36 & 199.89 & 208.59 & 209.16 & 210.13 & 212.51 \\ 216.86 & 223.43 & 233.91 & 238.56 & 249.46 & 269.47 \\ 271.50 & 279.91 & 291.25 & 293.11 & 306.71 & 306.95 \\ 316.86 & 335.18 & 342.90 & 348.22 & 357.19 & 359.38 \\ 361.27 & 365.09 & 384.66 & 409.10 & 412.25 & 418.05 \\ 418.96 & 420.60 & 421.41 & 449.15 & 457.77 & 562.88 \\ 592.35 & 610.19 & 611.03 & 652.36 & 655.11 & 656.66 \\ 664.27 & 671.18 & 672.38 & 691.42 & 692.98 & 693.96 \\ 694.56 & 698.81 & 716.72 & 724.59 & 734.62 & 736.14 \\ 764.53 & 771.32 & 771.68 & 777.10 & 780.30 & 785.13 \\ 785.35 & 799.31 & 842.65 & 850.92 & 853.45 & 855.76 \\ 861.06 & 866.98 & 886.43 & 891.70 & 892.52 & 894.52 \\ 994.33 & 1003.23 & 1004.03 & 1010.51 & 1016.20 & 1031.71 \\ 1032.64 & 1036.14 & 1041.32 & 1041.35 & 1042.68 & 1054.61 \\ 1060.87 & 1139.71 & 1139.88 & 1147.96 & 1150.04 & 1183.44 \\ 1184.04 & 1262.09 & 1264.21 & 1274.73 & 1282.22 & 1303.48 \\ 1327.92 & 1329.11 & 1339.87 & 1355.31 & 1364.21 & 1377.84 \\ 1383.96 & 1389.02 & 1392.30 & 1442.72 & 1467.93 & 1468.77 \\ 1470.46 & 1499.91 & 1501.81 & 1524.02 & 1555.20 & 1556.12 \\ 1576.42 & 1588.36 & 1605.69 & 1608.94 & 1660.18 & 2547.91 \\ 3122.51 & 3124.73 & 3125.07 & 3125.83 & 3154.68 & 3155.39 \\ 3155.59 & 3155.80 & 3173.71 & 3174.38 & 3174.51 & 3174.81\end{array}$

TURBOMOLE Energies and Thermochemistry ${ }^{1}$ RI-PBE/TZVP,SV(P) $-5710.973124$

ZPE 0.3208614

RI-PBE/TZVP,SV(P) + ZPE

$-5710.652263$

RI-PBE/TZVPP

B3-LYP/TZVP,SV(P)

$\mathrm{ZPE}(\mathrm{kJ} / \mathrm{mol})$

$-5713.462679$

$-5712.730492$

$\ln$ (qtrans)

842.4

20.43

In(qrot)

17.5

In(qvib)

34.38

chem.pot. $(\mathrm{kJ} / \mathrm{mol})$

663.17

energy $(\mathrm{kJ} / \mathrm{mol})$

934.23

entropy $(\mathrm{kJ} / \mathrm{mol} / \mathrm{K})$

0.91745

$\mathrm{Cv}(\mathrm{kJ} / \mathrm{mol}-\mathrm{K})$

0.5566893

Cp $((\mathrm{kJ} / \mathrm{mol}-\mathrm{K})$

0.5650036

3.77

$\mathrm{RI}-\mathrm{PBE} / \mathrm{TZVP}, \mathrm{SV}(\mathrm{P}) \quad\left\langle\mathrm{S}^{2}\right\rangle$

3.77

$\mathrm{RI}-\mathrm{PBE} / \mathrm{TZVPP}\left\langle\mathrm{S}^{2}>\right.$

3.80

Freeh output; 298.15K, 0.1MPa, unscaled 


\section{${ }^{4} \mathrm{~A}$}

\section{Hexachlorobenzene: $[($ Porphyrin $)(\mathrm{Fe}=\mathbf{O})(\mathrm{SH})$ Product Complex}

RI - PBE/TZVP, SV (P) Cartesian coordinates$$
52
$$$$
\text { Energy }=-5711.019173389
$$

\begin{tabular}{lrrr} 
Energy & -1.5326028 & 0.2935200 & 2.4548503 \\
$\mathrm{C}$ & -1.8357562 & -0.5310409 & 2.3410706 \\
$\mathrm{C}$ & -2.2927760 & -1.8082943 & 2.5765412 \\
$\mathrm{C}$ & -3.7191492 & -1.7952129 & 2.8168657 \\
$\mathrm{C}$ & -4.1207456 & -0.4856747 & 2.7289200 \\
$\mathrm{Fe}$ & 0.0804268 & 0.0220207 & 1.9461582 \\
$\mathrm{O}$ & -0.4572653 & -0.0563448 & 0.0384627 \\
$\mathrm{C}$ & -1.5029855 & -2.9582077 & 2.5884289 \\
$\mathrm{C}$ & -0.1411439 & -2.9878351 & 2.2918840 \\
$\mathrm{~N}$ & 0.6410231 & -1.8945441 & 1.9593065 \\
$\mathrm{C}$ & 1.8808096 & -2.4035325 & 1.6192993 \\
$\mathrm{C}$ & 1.8853049 & -3.8392929 & 1.7546954 \\
$\mathrm{C}$ & 0.6317464 & -4.2016034 & 2.1862789 \\
$\mathrm{C}$ & 2.9753111 & -1.6521739 & 1.1958688 \\
$\mathrm{C}$ & 2.9940902 & -0.2614339 & 1.1370421 \\
$\mathrm{~N}$ & 1.9377387 & 0.5668480 & 1.4472152 \\
$\mathrm{C}$ & 2.4456657 & 1.8518403 & 1.4075474 \\
$\mathrm{C}$ & 3.8403548 & 1.8314952 & 1.0329564 \\
$\mathrm{C}$ & 4.1778609 & 0.5171752 & 0.8447251 \\
$\mathrm{C}$ & 1.7343753 & 3.0109716 & 1.7040543 \\
$\mathrm{C}$ & 0.3725778 & 3.0462330 & 2.0077288 \\
$\mathrm{~N}$ & -0.4586074 & 1.9446306 & 2.0944014 \\
$\mathrm{C}$ & -1.7354898 & 2.4449616 & 2.2717001 \\
$\mathrm{C}$ & -1.7028765 & 3.8873539 & 2.3140403 \\
$\mathrm{C}$ & -0.3879544 & 4.2612036 & 2.1662159 \\
$\mathrm{C}$ & -2.8998480 & 1.6872411 & 2.4015538 \\
$\mathrm{~S}$ & 0.5617623 & -0.0303648 & 4.1184578 \\
$\mathrm{H}$ & -0.0515959 & 1.1448286 & 4.4739910 \\
$\mathrm{H}$ & 0.0392820 & 5.2722911 & 2.1362309 \\
$\mathrm{H}$ & -2.5879997 & 4.5252832 & 2.4382208 \\
$\mathrm{H}$ & -3.8506971 & 2.2283002 & 2.5197059 \\
$\mathrm{H}$ & -5.1273601 & -0.0650415 & 2.8542615 \\
$\mathrm{H}$ & -4.3242443 & -2.6875585 & 3.0250147 \\
$\mathrm{H}$ & -1.9963743 & -3.9163366 & 2.8102186 \\
$\mathrm{H}$ & 0.2419889 & -5.2069118 & 2.3947541 \\
$\mathrm{H}$ & 2.7489617 & -4.4814397 & 1.5373102 \\
$\mathrm{H}$ & 3.8971085 & -2.1902486 & 0.9304450 \\
$\mathrm{H}$ & 5.1431198 & 0.0888278 & 0.5458538 \\
$\mathrm{H}$ & 4.4653215 & 2.7262620 & 0.9155514 \\
$\mathrm{H}$ & 2.2709661 & 3.9698023 & 1.6491976 \\
$\mathrm{C}$ & -0.3466872 & -0.0639377 & -1.2316444 \\
$\mathrm{C}$ & -0.3921206 & 1.1623474 & -2.0239662 \\
$\mathrm{C}$ & -0.3386152 & -1.3045766 & -1.9906783 \\
$\mathrm{C}$ & -0.4540042 & -1.3176147 & -3.3848106 \\
$\mathrm{C}$ & -0.5318005 & 1.1355686 & -3.4252007 \\
$\mathrm{C}$ & -0.5525758 & -0.0980838 & -4.1108826 \\
$\mathrm{C} 1$ & -0.4692199 & 2.6538689 & -1.1781014 \\
$\mathrm{Cl}$ & -0.6450328 & 2.6159229 & -4.3100538 \\
$\mathrm{Cl}$ & -0.4733270 & -2.8205091 & -4.2400542 \\
$\mathrm{Cl}$ & -0.2668630 & -2.7796775 & -1.1076224 \\
$\mathrm{Cl}$ & -0.6894448 & -0.1300337 & -5.8280638 \\
$\mathrm{Cl}$ & 2.3556007 & 0.4207749 & -1.9502440 \\
& & & \\
\hline
\end{tabular}

Vibrational frequencies

$\begin{array}{rrrrrr}0.00 & 0.00 & 0.00 & 0.00 & 0.00 & 0.00 \\ 8.25 & 15.59 & 30.63 & 41.31 & 49.76 & 50.68 \\ 55.47 & 60.89 & 70.89 & 77.47 & 80.94 & 90.12 \\ 96.97 & 106.74 & 108.78 & 126.64 & 142.16 & 148.36 \\ 152.44 & 171.49 & 191.09 & 207.29 & 208.81 & 212.08 \\ 215.03 & 220.65 & 231.02 & 248.72 & 260.14 & 267.37 \\ 269.73 & 274.60 & 276.25 & 282.63 & 288.36 & 301.94 \\ 325.00 & 326.84 & 333.99 & 349.67 & 358.66 & 358.99 \\ 361.05 & 371.90 & 376.36 & 399.33 & 401.94 & 414.32 \\ 416.74 & 423.11 & 423.63 & 453.50 & 502.90 & 524.47 \\ 549.83 & 598.32 & 637.40 & 644.28 & 651.20 & 653.51 \\ 669.05 & 673.80 & 680.44 & 692.11 & 693.78 & 694.39 \\ 709.72 & 716.44 & 722.69 & 730.53 & 734.59 & 760.37 \\ 768.80 & 772.71 & 777.13 & 780.04 & 780.33 & 782.60 \\ 786.33 & 797.88 & 834.23 & 843.70 & 844.48 & 852.32 \\ 864.43 & 889.16 & 893.17 & 895.30 & 896.63 & 985.69 \\ 993.60 & 995.07 & 1000.69 & 1013.79 & 1018.20 & 1030.10 \\ 1032.83 & 1037.19 & 1041.86 & 1042.95 & 1043.41 & 1053.59 \\ 1109.40 & 1137.26 & 1141.11 & 1143.68 & 1180.92 & 1184.28 \\ 1248.19 & 1259.45 & 1262.35 & 1269.74 & 1317.03 & 1329.14 \\ 1331.87 & 1345.19 & 1348.31 & 1362.85 & 1365.10 & 1372.71 \\ 1377.64 & 1385.76 & 1433.66 & 1449.90 & 1464.41 & 1468.65 \\ 1476.59 & 1501.06 & 1511.76 & 1518.28 & 1547.61 & 1556.63 \\ 1579.26 & 1594.65 & 1614.96 & 1623.67 & 1664.68 & 2543.63 \\ 3128.11 & 3128.20 & 3130.81 & 3134.64 & 3156.70 & 3157.61 \\ 3157.74 & 3162.93 & 3175.46 & 3176.02 & 3176.34 & 3182.03\end{array}$

TURBOMOLE Energies and Thermochemistry ${ }^{1}$

\begin{tabular}{|c|c|}
\hline RI-PBE/TZVP,SV(P) & -5711.019173 \\
\hline ZPE & 0.3217053 \\
\hline RI-PBE/TZVP,SV(P) + ZPE & -5710.697468 \\
\hline RI-PBE/TZVPP & -5713.505922 \\
\hline \multicolumn{2}{|l|}{ B3-LYP/TZVP,SV(P) } \\
\hline $\mathrm{ZPE}(\mathrm{kJ} / \mathrm{mol})$ & 844.6 \\
\hline In(qtrans) & 20.43 \\
\hline In(qrot) & 17.59 \\
\hline $\ln (q v i b)$ & 36.32 \\
\hline chem.pot. (kJ/mol) & 660.37 \\
\hline energy (kJ/mol) & 939.18 \\
\hline entropy $(\mathrm{kJ} / \mathrm{mol} / \mathrm{K})$ & 0.94347 \\
\hline $\mathrm{Cv}(\mathrm{kJ} / \mathrm{mol}-\mathrm{K})$ & 0.5645389 \\
\hline $\mathrm{Cp}((\mathrm{kJ} / \mathrm{mol}-\mathrm{K})$ & 0.5728532 \\
\hline $\mathrm{RI}-\mathrm{PBE} / \mathrm{TZVP}, \mathrm{SV}(\mathrm{P}) \quad<\mathrm{S}^{2}>$ & 3.79 \\
\hline RI - $\mathrm{PBE} / \mathrm{TZVPP}<\mathrm{S}^{2}>$ & 3.79 \\
\hline B3 - LYP / TZVP , SV (P) $\quad\left\langle\mathrm{S}^{2}>\right.$ & 116.7 \\
\hline
\end{tabular}

${ }^{1}$ Freeh output; $298.15 \mathrm{~K}, 0.1 \mathrm{MPa}$, unscaled 


\section{${ }^{4} \mathrm{~A}[($ Porphyrin $)(\mathbf{F e})(\mathrm{SH})]$}

\section{RI - PBE/TZVP, SV(P) Cartesian coordinates}

39

Energy $=-2648.560291286$

$\begin{array}{lrrr}\text { C } & -2.9384858 & 0.7846801 & -0.2273174 \\ \mathrm{~N} & -1.9862763 & -0.2190028 & -0.2241805 \\ \mathrm{C} & -2.7018119 & -1.4019708 & -0.2420168 \\ \mathrm{C} & -4.1219135 & -1.1396904 & -0.2527946 \\ \mathrm{C} & -4.2695197 & 0.2257084 & -0.2423825 \\ \mathrm{Fe} & 0.0034522 & -0.0002770 & 0.0197922 \\ \mathrm{C} & -2.1475606 & -2.6820279 & -0.2650027 \\ \mathrm{C} & -0.7794190 & -2.9483884 & -0.2813240 \\ \mathrm{~N} & 0.2211043 & -1.9968587 & -0.2526150 \\ \mathrm{C} & 1.4035749 & -2.7120642 & -0.2594145 \\ \mathrm{C} & 1.1438478 & -4.1343187 & -0.3065568 \\ \mathrm{C} & -0.2194194 & -4.2817650 & -0.3212094 \\ \mathrm{C} & 2.6817041 & -2.1575309 & -0.2286448 \\ \mathrm{C} & 2.9478446 & -0.7883812 & -0.2102615 \\ \mathrm{~N} & 1.9947682 & 0.2155153 & -0.2075668 \\ \mathrm{C} & 2.7110796 & 1.3993475 & -0.2306320 \\ \mathrm{C} & 4.1308111 & 1.1366929 & -0.2390696 \\ \mathrm{C} & 4.2783203 & -0.2289818 & -0.2252419 \\ \mathrm{C} & 2.1574622 & 2.6797324 & -0.2616673 \\ \mathrm{C} & 0.7892733 & 2.9472228 & -0.2816475 \\ \mathrm{~N} & -0.2108141 & 1.9954386 & -0.2531791 \\ \mathrm{C} & -1.3941889 & 2.7092834 & -0.2671584 \\ \mathrm{C} & -1.1349157 & 4.1314825 & -0.3157713 \\ \mathrm{C} & 0.2284128 & 4.2800335 & -0.3268221 \\ \mathrm{C} & -2.6720461 & 2.1537771 & -0.2433524 \\ \mathrm{~S} & -0.0859014 & -0.0064837 & 2.3622759 \\ \mathrm{H} & 1.1162082 & 0.6307741 & 2.5358074 \\ \mathrm{H} & 0.8197954 & 5.2047754 & -0.3618863 \\ \mathrm{H} & -1.9117572 & 4.9074912 & -0.3413389 \\ \mathrm{H} & -3.5305417 & 2.8423017 & -0.2504013 \\ \mathrm{H} & -5.1948051 & 0.8170805 & -0.2508542 \\ \mathrm{H} & -4.9002263 & -1.9144964 & -0.2706946 \\ \mathrm{H} & -2.8381825 & -3.5386775 & -0.2814949 \\ \mathrm{H} & -0.8114701 & -5.2060584 & -0.3557252 \\ \mathrm{H} & 1.9204952 & -4.9106827 & -0.3273673 \\ \mathrm{H} & 3.5401760 & -2.8461112 & -0.2323971 \\ \mathrm{H} & 5.2037527 & -0.8201353 & -0.2342165 \\ \mathrm{H} & 4.9093092 & 1.9113083 & -0.2602017 \\ \mathrm{H} & 2.8487346 & 3.5358515 & -0.2817602\end{array}$

Vibrational frequencies

$\begin{array}{rrrrrr}0.00 & 0.00 & 0.00 & 0.00 & 0.00 & 0.00 \\ 35.89 & 37.90 & 73.79 & 78.89 & 92.08 & 98.86 \\ 141.46 & 141.90 & 202.18 & 211.27 & 227.10 & 236.29 \\ 238.32 & 239.49 & 271.12 & 273.91 & 280.31 & 302.73 \\ 335.70 & 351.78 & 353.19 & 354.79 & 396.06 & 399.74 \\ 415.97 & 416.90 & 421.05 & 421.87 & 458.17 & 497.96 \\ 653.07 & 658.57 & 660.15 & 675.59 & 686.61 & 699.63 \\ 700.26 & 701.24 & 720.21 & 724.50 & 735.03 & 736.70 \\ 765.02 & 773.07 & 774.49 & 782.18 & 783.89 & 785.09 \\ 787.91 & 799.44 & 836.31 & 845.74 & 846.51 & 854.64 \\ 890.74 & 894.49 & 895.26 & 896.93 & 990.03 & 995.96 \\ 998.87 & 1008.64 & 1009.76 & 1029.58 & 1030.31 & 1035.80 \\ 1039.11 & 1039.36 & 1041.12 & 1052.10 & 1137.52 & 1144.00 \\ 1144.26 & 1180.62 & 1182.62 & 1258.70 & 1260.52 & 1325.46 \\ 1329.86 & 1335.27 & 1348.77 & 1365.87 & 1375.88 & 1378.25 \\ 1379.25 & 1384.20 & 1470.18 & 1472.33 & 1476.64 & 1508.73 \\ 1521.65 & 1553.79 & 1559.05 & 1581.42 & 1607.97 & 1615.00 \\ 1623.11 & 1665.46 & 2550.16 & 3125.20 & 3125.52 & 3125.66 \\ 3126.07 & 3153.77 & 3153.92 & 3154.00 & 3154.43 & 3172.83 \\ 3173.03 & 3173.18 & 3173.43 & & & \end{array}$

TURBOMOLE Energies and Thermochemistry ${ }^{1}$

\begin{tabular}{|c|c|}
\hline RI - PBE/TZVP , SV (P) & -2648.560291 \\
\hline $\mathrm{ZPE}$ & 0.2765425 \\
\hline $\mathrm{RI}-\mathrm{PBE} / \mathrm{TZVP}, \mathrm{SV}(\mathrm{P})+\mathrm{ZPE}$ & -2648.283749 \\
\hline RI - PBE/TZVPP & -2649.769223 \\
\hline B3 - LYP / TZVP , SV (P) & -2649.397186 \\
\hline $\mathrm{ZPE} \quad(\mathrm{kJ} / \mathrm{mol})$ & 726.1 \\
\hline $\ln ($ qtrans $)$ & 19.58 \\
\hline $\ln ($ qrot $)$ & 16.11 \\
\hline $\ln ($ qvib) & 15.83 \\
\hline chem.pot. (kJ/mol) & 598.35 \\
\hline energy $(\mathrm{kJ} / \mathrm{mol})$ & 780.51 \\
\hline entropy $(\mathrm{kJ} / \mathrm{mol} / \mathrm{K})$ & 0.61928 \\
\hline $\mathrm{CV} \quad(\mathrm{kJ} / \mathrm{mol}-\mathrm{K})$ & 0.3525444 \\
\hline $\mathrm{Cp} \quad((\mathrm{kJ} / \mathrm{mol}-\mathrm{K})$ & 0.3608587 \\
\hline RI - PBE/TZVP, SV (P) & 3.78 \\
\hline $\mathrm{RI}-\mathrm{PBE} / \mathrm{TZVPP} \quad<\mathrm{S}^{2}>$ & 3.78 \\
\hline B3 - LYP / TZVP , SV (P) $<\mathrm{S}^{2}>$ & 3.79 \\
\hline
\end{tabular}

${ }^{1}$ Freeh output; $298.15 \mathrm{~K}, 0.1 \mathrm{MPa}$, unscaled 


\section{Pentachlorophenol}

$\begin{array}{lrrr}13 & & \\ \text { Energy }=-2603.216012700 & \\ \text { C } & -0.4949292 & -1.2199985 & 0.0020341 \\ \text { C } & 0.9130329 & -1.2142307 & -0.0024165 \\ \text { C } & 1.6516949 & -0.0085780 & 0.0027918 \\ \text { C } & 0.9432383 & 1.2157140 & -0.0023637 \\ \text { C } & -0.4681966 & 1.2247797 & 0.0007968 \\ \text { C } & -1.1935286 & 0.0085634 & -0.0013123 \\ \text { O } & 2.9973880 & 0.0095524 & 0.0066633 \\ \text { H } & 3.3000064 & -0.9345609 & 0.0061544 \\ \text { Cl } & 1.8514024 & 2.6853387 & -0.0027772 \\ \text { C1 } & -1.3120696 & 2.7313061 & 0.0007404 \\ \text { Cl } & -2.9221609 & 0.0222821 & 0.0012434 \\ \text { Cl } & -1.3509610 & -2.7199123 & -0.0012929 \\ \text { Cl } & 1.8294784 & -2.6988722 & -0.0009365\end{array}$

Vibrational frequencies

$\begin{array}{rrrrrr}0.00 & 0.00 & 0.00 & 0.00 & 0.00 & 0.00 \\ 71.16 & 78.36 & 112.27 & 167.54 & 198.58 & 203.33 \\ 208.58 & 213.41 & 301.16 & 317.43 & 331.00 & 332.84 \\ 334.60 & 372.95 & 458.39 & 522.15 & 540.46 & 560.07 \\ 624.72 & 636.49 & 695.54 & 761.12 & 869.62 & 980.56 \\ 1115.43 & 1231.49 & 1272.74 & 1348.97 & 1381.53 & 1430.81 \\ 1552.76 & 1571.77 & 3398.11 & & & \end{array}$

TURBOMOLE Energies and Thermochemistry ${ }^{1}$

\begin{tabular}{lr}
\hline RI-PBE/TZVP,SV(P) & -2603.216013 \\
\hline ZPE & 0.0551224 \\
RI-PBE/TZVP,SV(P) + ZPE & -2603.16089 \\
RI-PBE/TZVPP & -2604.356142 \\
B3-LYP/TZVP,SV(P) & -2604.051221 \\
ZPE (kJ/mol) & 144.7 \\
In(qtrans) & 18.98 \\
In(qrot) & 15.06 \\
In(qvib) & 7.61 \\
chem.pot. (kJ/mol) & 41.48 \\
energy $(\mathrm{kJ} / \mathrm{mol})$ & 175.56 \\
entropy $(\mathrm{kJ} / \mathrm{mol} / \mathrm{K})$ & 0.45803 \\
Cv $(\mathrm{kJ} / \mathrm{mol}-\mathrm{K})$ & 0.17167 \\
Cp $((\mathrm{kJ} / \mathrm{mol}-\mathrm{K})$ & 0.1799843 \\
\hline
\end{tabular}

${ }^{1}$ Freeh output; $298.15 \mathrm{~K}, 0.1 \mathrm{MPa}$, unscaled 
${ }^{2} \mathbf{A}$

\section{Pentachlorophenol: $[($ Porphyrin $)(\mathrm{Fe}=\mathbf{O})(\mathrm{SH})$ Prereactant Complex (para-oxidation)}

RI-PBE/TZVP, SV (P) Cartesian coordinates

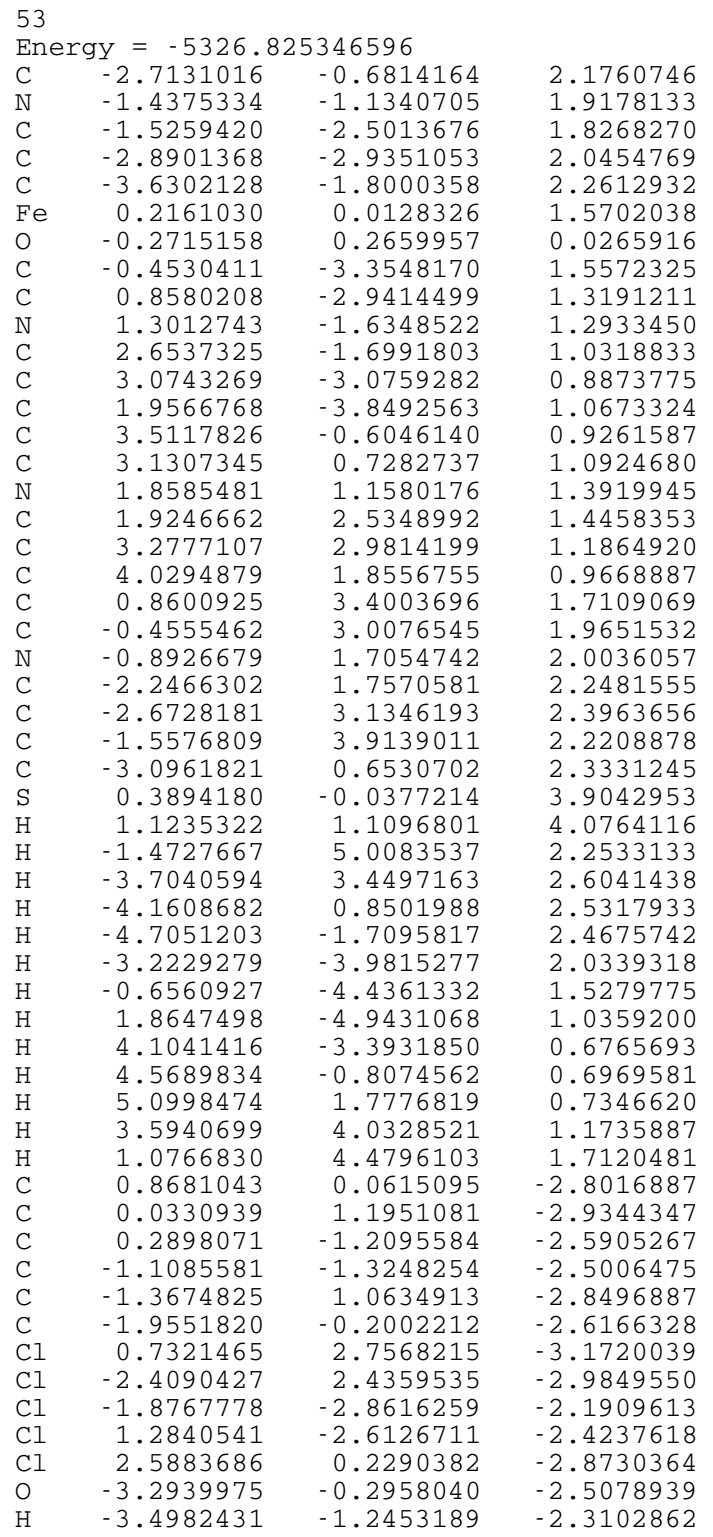

Vibrational frequencies

$\begin{array}{rrrrrr}17.02 & 0.00 & 0.00 & 0.00 & 0.00 & 0.00 \\ 0.00 & 7.58 & 17.97 & 20.60 & 21.63 & 42.24 \\ 42.45 & 55.69 & 73.80 & 81.26 & 94.92 & 99.58 \\ 103.06 & 113.78 & 117.06 & 146.54 & 148.92 & 171.46 \\ 177.13 & 199.08 & 199.80 & 203.62 & 205.86 & 208.94 \\ 213.70 & 231.67 & 239.18 & 271.65 & 273.28 & 274.20 \\ 280.40 & 286.08 & 294.17 & 300.86 & 308.53 & 322.14 \\ 331.21 & 333.13 & 337.25 & 344.99 & 358.22 & 358.90 \\ 364.35 & 372.26 & 395.38 & 399.92 & 417.91 & 419.44 \\ 419.47 & 421.67 & 458.07 & 459.24 & 500.47 & 540.69 \\ 568.96 & 595.63 & 632.75 & 636.26 & 649.74 & 656.58 \\ 657.01 & 672.94 & 679.82 & 694.26 & 694.46 & 695.38 \\ 697.67 & 715.71 & 723.47 & 734.00 & 734.92 & 758.61 \\ 763.85 & 771.80 & 772.29 & 778.33 & 781.93 & 784.19 \\ 784.49 & 798.30 & 840.57 & 849.71 & 850.38 & 850.48 \\ 858.46 & 867.17 & 886.97 & 892.18 & 892.49 & 894.46 \\ 977.20 & 992.97 & 1001.74 & 1002.38 & 1011.54 & 1014.82 \\ 1032.47 & 1032.60 & 1036.47 & 1041.44 & 1042.41 & 1042.83 \\ 1054.90 & 1112.16 & 1139.75 & 1147.45 & 1148.87 & 1182.17 \\ 1183.72 & 1237.20 & 1261.02 & 1262.71 & 1272.25 & 1315.87 \\ 1329.67 & 1331.28 & 1340.98 & 1355.65 & 1364.04 & 1376.13 \\ 1383.71 & 1386.05 & 1391.50 & 1392.71 & 1433.26 & 1470.00 \\ 1471.68 & 1473.25 & 1505.08 & 1525.28 & 1556.48 & 1557.97 \\ 1558.54 & 1574.67 & 1579.42 & 1603.78 & 1608.93 & 1610.06 \\ 1661.64 & 2542.77 & 3122.53 & 3123.74 & 3125.27 & 3125.83 \\ 3154.80 & 3155.22 & 3155.90 & 3156.51 & 3173.85 & 3174.19 \\ 3175.01 & 3175.50 & 3399.84 & & & \end{array}$

TURBOMOLE Energies and Thermochemistry ${ }^{1}$

RI-PBE/TZVP,SV(P) $\quad-5326.825347$

\section{ZPE} 0.3356817

RI-PBE/TZVP,SV(P) + ZPE

RI-PBE/TZVPP

B3-LYP/TZVP,SV(P)

$\mathrm{ZPE}(\mathrm{kJ} / \mathrm{mol})$

$-5326.489665$

$-5329.258734$

$-5328.492939$

$\ln$ (qtrans)

881.3

20.39

In(qrot)

17.44

In(qvib)

34.98

chem.pot. $(\mathrm{kJ} / \mathrm{mol})$

700.86

energy $(\mathrm{kJ} / \mathrm{mol})$

972.65

entropy $(\mathrm{kJ} / \mathrm{mol} / \mathrm{K})$

0.9199

$\mathrm{Cv}(\mathrm{kJ} / \mathrm{mol}-\mathrm{K})$

$\mathrm{Cp}((\mathrm{kJ} / \mathrm{mol}-\mathrm{K})$

0.5561261

0.5644404

$\mathrm{RI}-\mathrm{PBE} / \mathrm{TZVP}, \mathrm{SV}(\mathrm{P}) \quad\left\langle\mathrm{S}^{2}>\right.$

1.09

$\mathrm{RI}-\mathrm{PBE} / \mathrm{TZVPP}\left\langle\mathrm{S}^{2}\right\rangle \quad 1.03$

B3 - LYP/TZVP, SV (P) $<\mathrm{S}^{2}>$

1.63

${ }^{1}$ Freeh output; $298.15 \mathrm{~K}, 0.1 \mathrm{MPa}$ unscaled 
${ }^{2} \mathbf{A}$

\section{Pentachlorophenol:[(Porphyrin $)(\mathrm{Fe}=\mathbf{O})(\mathrm{SH})$ Transition State (para-oxidation)}

\begin{tabular}{|c|c|c|c|}
\hline \multicolumn{2}{|c|}{ RI - PBE/TZVP, SV (P) } & Cartesian & coordinates \\
\hline 53 & & & \\
\hline & $g y=-5326.8$ & 3114368 & \\
\hline $\mathrm{C}$ & -2.6890264 & -1.8731077 & 0.2834674 \\
\hline $\mathrm{N}$ & -1.4912294 & -1.7226460 & 0.9527184 \\
\hline C & -1.0400817 & -2.9928639 & 1.2139995 \\
\hline $\mathrm{C}$ & -1.9653509 & -3.9783752 & 0.6952941 \\
\hline $\mathrm{C}$ & -2.9939780 & -3.2795068 & 0.1161164 \\
\hline $\mathrm{Fe}$ & -0.5637998 & 0.0292405 & 1. 3947264 \\
\hline $\mathrm{O}$ & -0.0302880 & 0.2044927 & -0.2525111 \\
\hline $\mathrm{C}$ & 0.1356719 & -3.2939038 & 1.9068042 \\
\hline $\mathrm{C}$ & 1.0000841 & -2.3445628 & 2.4499280 \\
\hline $\mathrm{N}$ & 0.8665625 & -0.9738075 & 2.3377127 \\
\hline $\mathrm{C}$ & 1.8959503 & -0.4370307 & 3.0898039 \\
\hline $\mathrm{C}$ & 2.7073359 & -1.4894015 & 3.6607721 \\
\hline C & 2.1542478 & -2.6768363 & 3.2566189 \\
\hline $\mathrm{C}$ & 2.1491958 & 0.9208300 & 3.2715234 \\
\hline C & 1.3876289 & 1.9545607 & 2.7214460 \\
\hline $\mathrm{N}$ & 0.2609855 & 1.7802872 & 1.9539742 \\
\hline C & -0.1699615 & 3.0458485 & 1.6175104 \\
\hline $\mathrm{C}$ & 0.7191457 & 4.0429490 & 2.1766192 \\
\hline C & 1.6854586 & 3.3626348 & 2.8736320 \\
\hline C & -1.3203844 & 3.3548598 & 0.8864910 \\
\hline $\mathrm{C}$ & -2.2475551 & 2.4258494 & 0.4091312 \\
\hline $\mathrm{N}$ & -2.1523181 & 1.0649875 & 0.5710891 \\
\hline C & -3.2462492 & 0.5229141 & -0.0565855 \\
\hline $\mathrm{C}$ & -4.0813119 & 1.5746318 & -0.6064203 \\
\hline $\mathrm{C}$ & -3.4562436 & 2.7612499 & -0.3200195 \\
\hline C & -3.5075161 & -0.8434359 & -0.1833802 \\
\hline $\mathrm{S}$ & -1.9033007 & 0.0383918 & 3.2711991 \\
\hline $\mathrm{H}$ & -2.1537882 & 1. 3844644 & 3.3467198 \\
\hline $\mathrm{H}$ & -3.7713422 & 3.7824849 & -0.5730226 \\
\hline $\mathrm{H}$ & -5.0222114 & 1.4074130 & -1.1476612 \\
\hline $\mathrm{H}$ & -4.4304835 & -1.1346930 & -0.7081966 \\
\hline $\mathrm{H}$ & -3.8933572 & -3.6668101 & -0.3812951 \\
\hline $\mathrm{H}$ & -1.8334357 & -5.0654256 & 0.7791368 \\
\hline $\mathrm{H}$ & 0.3778911 & -4.3555397 & 2.0674327 \\
\hline $\mathrm{H}$ & 2.4768414 & -3.7005406 & 3.4891408 \\
\hline $\mathrm{H}$ & 3.5873686 & -1.3209193 & 4.2956188 \\
\hline $\mathrm{H}$ & 3.0183407 & 1.1986008 & 3.8870994 \\
\hline $\mathrm{H}$ & 2.5340091 & 3.7640870 & 3.4434687 \\
\hline $\mathrm{H}$ & 0.5973770 & 5.1272342 & 2.0508462 \\
\hline $\mathrm{H}$ & -1.5239236 & 4.4196199 & 0.6941713 \\
\hline $\mathrm{C}$ & 1.5135918 & 0.0943096 & -1.2157657 \\
\hline $\mathrm{C}$ & 1.2460234 & 2130255 & -2.1115543 \\
\hline C & 1.3366275 & -1.2346981 & -1.7709941 \\
\hline C & 0.5953707 & -1.3968468 & -2.9447112 \\
\hline $\mathrm{C}$ & 0.5248097 & 1.0244417 & -3.2899941 \\
\hline $\mathrm{C}$ & 0.1779949 & -0.2876643 & -3.7205626 \\
\hline $\mathrm{Cl}$ & 1.6614005 & 2.7990465 & -1.5999708 \\
\hline $\mathrm{Cl}$ & 0.0466565 & 2.3539159 & -4.2864687 \\
\hline $\mathrm{Cl}$ & 0.1836180 & -2.9791343 & -3.5595488 \\
\hline $\mathrm{Cl}$ & 1.9377397 & -2.6078456 & -0.9358356 \\
\hline $\mathrm{Cl}$ & 2.8492931 & 0.3029301 & -0.0982108 \\
\hline 0 & -0.5280189 & -0.4370915 & -4.8515938 \\
\hline $\mathrm{H}$ & -0.6665648 & -1.4127454 & -4.9772609 \\
\hline
\end{tabular}

$\begin{array}{rrrrrr}1515.80 & 0.00 & 0.00 & 0.00 & 0.00 & 0.00 \\ 0.00 & 6.62 & 24.25 & 33.18 & 43.04 & 47.69 \\ 51.18 & 57.54 & 72.77 & 77.56 & 81.87 & 94.35 \\ 103.91 & 115.35 & 120.62 & 139.59 & 145.17 & 151.00 \\ 169.00 & 197.21 & 200.11 & 205.87 & 207.29 & 212.92 \\ 224.59 & 231.31 & 254.76 & 256.94 & 263.70 & 272.91 \\ 279.80 & 285.49 & 294.95 & 295.92 & 311.85 & 325.64 \\ 331.71 & 337.33 & 346.58 & 350.33 & 357.25 & 359.25 \\ 365.84 & 388.26 & 394.87 & 413.89 & 418.01 & 420.33 \\ 422.06 & 424.15 & 458.91 & 459.57 & 511.09 & 546.14 \\ 555.55 & 627.99 & 633.34 & 641.43 & 651.81 & 656.75 \\ 657.87 & 673.21 & 680.17 & 688.60 & 694.93 & 695.10 \\ 697.67 & 698.49 & 717.50 & 723.97 & 734.19 & 735.60 \\ 744.28 & 763.42 & 770.41 & 771.45 & 777.10 & 783.03 \\ 784.86 & 785.21 & 798.74 & 838.54 & 847.31 & 847.86 \\ 855.40 & 865.23 & 884.93 & 889.73 & 890.68 & 892.28 \\ 974.18 & 991.36 & 1000.82 & 1001.51 & 1010.39 & 1013.41 \\ 1030.67 & 1031.96 & 1035.19 & 1040.35 & 1041.09 & 1041.38 \\ 1053.88 & 1090.71 & 1138.84 & 1147.16 & 1148.18 & 1181.97 \\ 1182.97 & 1188.05 & 1216.85 & 1261.18 & 1261.95 & 1311.74 \\ 1322.04 & 1329.41 & 1331.91 & 1341.79 & 1363.63 & 1374.21 \\ 1384.00 & 1391.63 & 1392.36 & 1393.54 & 1420.97 & 1470.27 \\ 1471.03 & 1473.30 & 1504.84 & 1505.18 & 1524.34 & 1556.85 \\ 1557.25 & 1559.31 & 1579.84 & 1606.78 & 1611.66 & 1614.25 \\ 1661.23 & 2550.78 & 3119.65 & 3121.16 & 3123.60 & 3123.69 \\ 3152.99 & 3153.37 & 3153.64 & 3153.91 & 3172.25 & 3172.60 \\ 3172.90 & 3173.25 & 3365.94 & & & \end{array}$

TURBOMOLE Energies and Thermochemistry ${ }^{1}$

\begin{tabular}{lr}
\hline RI-PBE/TZVP,SV(P) & -5326.803114 \\
\hline ZPE & 0.3348069 \\
RI-PBE/TZVP,SV(P) + ZPE & -5326.468307 \\
RI-PBE/TZVPP & -5329.233533 \\
B3-LYP/TZVP,SV(P) & -5328.462356 \\
ZPE (kJ/mol) & 879 \\
In(qtrans) & 20.39 \\
In(qrot) & 17.39 \\
In(qvib) & 33.7 \\
chem.pot. $(\mathrm{kJ} / \mathrm{mol})$ & 701.83 \\
energy $(\mathrm{kJ} / \mathrm{mol})$ & 970.24 \\
entropy $(\mathrm{kJ} / \mathrm{mol} / \mathrm{K})$ & 0.90854 \\
CV $(\mathrm{kJ} / \mathrm{mol}-\mathrm{K})$ & 0.5572799 \\
Cp $((\mathrm{kJ} / \mathrm{mol}-\mathrm{K})$ & 0.5655942 \\
$\mathrm{RI}-\mathrm{PBE} / \mathrm{TZVP}, \mathrm{SV}(\mathrm{P}) \quad \mathrm{CS}^{2}>$ & 0.92 \\
$\mathrm{RI}-\mathrm{PBE} / \mathrm{TZVPP}<\mathrm{S}^{2}>$ & 0.90 \\
$\mathrm{~B} 3-\mathrm{LYP} / \mathrm{TZVP}, \mathrm{SV}(\mathrm{P}) \quad \mathrm{S}^{2}>$ & 1.45 \\
\hline
\end{tabular}

${ }^{1}$ Freeh output; $298.15 \mathrm{~K}, 0.1 \mathrm{MPa}$, unscaled 
${ }^{2} \mathbf{A}$

\section{Pentachlorophenol: $[($ Porphyrin $)(\mathrm{Fe}=\mathbf{O})(\mathrm{SH})$ Product Complex (para-oxidation)}

RI-PBE/TZVP, SV (P) Cartesian coordinates

$$
53
$$$$
\text { Energy }=-5326.862940781
$$

\begin{tabular}{|c|c|c|c|}
\hline $\mathrm{C}$ & -2.9665075 & -0.8919618 & 1.4866430 \\
\hline $\mathrm{N}$ & -1.6481005 & -1.2816373 & 1.5863618 \\
\hline C & -1.6538487 & -2.6571486 & 1.5028518 \\
\hline $\mathrm{C}$ & -3.0082102 & -3.1485082 & 1.3519072 \\
\hline C & -3.8255386 & -2.0474177 & 1.3292633 \\
\hline $\mathrm{Fe}$ & -0.0287161 & -0.1064596 & 1.8346630 \\
\hline 0 & 0.0394673 & 0.1626512 & -0.1702902 \\
\hline C & -0.5220774 & -3.4737608 & 1.5007466 \\
\hline C & 0.7960790 & -3.0166684 & 1.5410257 \\
\hline $\mathrm{N}$ & 1.1855819 & -1.6976308 & 1.6394121 \\
\hline $\mathrm{C}$ & 2.5637652 & -1.7070626 & 1.6061946 \\
\hline C & 3.0575618 & -3.0611321 & 1.4709854 \\
\hline C & 1.9555423 & -3.8779172 & 1.4410778 \\
\hline C & 3.3801269 & -0.5807092 & 1.7263628 \\
\hline $\mathrm{C}$ & 2.9115340 & 0.7134343 & 1.9598846 \\
\hline $\mathrm{N}$ & 1.5820888 & 1.0757772 & 2.0459284 \\
\hline C & 1.5780555 & 2.4207466 & 2.3548121 \\
\hline C & 2.9334777 & 2.9181635 & 2.4600696 \\
\hline C & 3.7646404 & 1.8576870 & 2.2007897 \\
\hline C & 0.4404400 & 3. 2162067 & 2.5029451 \\
\hline C & -0.8704589 & 2.7829777 & 2.2970060 \\
\hline $\mathrm{N}$ & -1.2454414 & 1.4976908 & 1.9697261 \\
\hline C & -2.6156907 & 1.5307863 & 1.821029 \\
\hline C & -3.1156647 & 2.8726315 & 2.034796 \\
\hline C & -2.0288606 & 3.6499601 & 2.346012 \\
\hline C & -3.4263705 & 0.4237038 & 1.563915 \\
\hline S & -0.1679119 & -0.4145445 & 3.993796 \\
\hline $\begin{array}{l}\mathrm{N} \\
\mathrm{H}\end{array}$ & 1.1736506 & -0.4953317 & 4.2848320 \\
\hline $\mathrm{H}$ & -1.9962423 & 4.7219831 & 2.5826605 \\
\hline $\mathrm{H}$ & -4.1724963 & 3.1630393 & 1.9661623 \\
\hline $\mathrm{H}$ & -4.5097489 & 0.5936413 & 1.4692334 \\
\hline $\mathrm{H}$ & -4.9179309 & -2.0030661 & 1.2250409 \\
\hline $\mathrm{H}$ & -3.2802340 & -4.2088829 & 1.2641888 \\
\hline $\mathrm{H}$ & -0.6801018 & -4.5601460 & 1.4206562 \\
\hline $\mathrm{H}$ & 1.9131535 & -4.9716675 & 1.3507455 \\
\hline $\mathrm{H}$ & 4.1195135 & -3.3355278 & 1.416195 \\
\hline $\mathrm{H}$ & 4.4696858 & -0.7307887 & 1.68281 \\
\hline $\mathrm{H}$ & 4.8623888 & 1.8328024 & 2.18018 \\
\hline $\mathrm{H}$ & 3.1995590 & 3.9581307 & 2.6926187 \\
\hline $\mathrm{H}$ & 0.5917638 & 4.2764748 & 2.7580488 \\
\hline C & 0.0538784 & 0.2891037 & -1.4019801 \\
\hline C & 0.1178836 & 1.5861957 & -2.0621513 \\
\hline $\mathrm{C}$ & -0.0038525 & -0.9800104 & -2.2996180 \\
\hline C & 0.0425290 & -0.7265870 & -3.7605644 \\
\hline C & 0.1463451 & 1.7007924 & -3.4429718 \\
\hline $\mathrm{C}$ & 0.1087676 & 0.5294072 & -4.3109627 \\
\hline $\mathrm{Cl}$ & 0.1642965 & 2.9773486 & -1.050712 \\
\hline $\mathrm{Cl}$ & 0.2266104 & 3.2357915 & -4.2115873 \\
\hline $\mathrm{Cl}$ & -0.00518 & -2.0944296 & -4.84102 \\
\hline $\mathrm{Cl}$ & -1.5618954 & -1.8377020 & -1.89050 \\
\hline $\mathrm{Cl}$ & 1.3 & -2.0363932 & -1.82617 \\
\hline O & & 0.7591567 & -5.63667 \\
\hline $\mathrm{H}$ & 0 . & -0.1273399 & -6.08075 \\
\hline
\end{tabular}

Vibrational frequencies

$\begin{array}{rrrrrr}17.81 & 0.00 & 0.00 & 0.00 & 0.00 & 0.00 \\ 0.00 & 19.42 & 22.02 & 32.79 & 50.07 & 52.37 \\ 53.65 & 56.72 & 70.54 & 89.94 & 95.96 & 104.28 \\ 112.57 & 119.03 & 136.18 & 146.78 & 148.93 & 161.31 \\ 170.27 & 187.90 & 207.46 & 210.46 & 222.69 & 226.97 \\ 231.43 & 257.24 & 258.79 & 267.51 & 274.25 & 278.87 \\ 282.78 & 286.73 & 303.93 & 313.67 & 317.10 & 330.98 \\ 340.27 & 341.79 & 359.09 & 359.43 & 360.31 & 385.33 \\ 391.46 & 400.05 & 411.51 & 419.95 & 420.77 & 421.85 \\ 421.94 & 438.76 & 460.34 & 477.92 & 487.46 & 538.02 \\ 613.06 & 637.72 & 652.02 & 657.31 & 657.62 & 664.74 \\ 673.67 & 679.03 & 685.19 & 689.06 & 696.10 & 697.42 \\ 698.80 & 720.21 & 722.16 & 727.18 & 734.57 & 735.91 \\ 760.70 & 769.07 & 769.37 & 776.43 & 784.29 & 785.17 \\ 785.92 & 799.25 & 802.46 & 834.20 & 842.34 & 842.88 \\ 849.99 & 884.49 & 889.13 & 889.66 & 891.40 & 895.32 \\ 928.68 & 992.31 & 999.24 & 1003.20 & 1009.30 & 1011.01 \\ 1030.08 & 1031.75 & 1034.81 & 1040.41 & 1041.29 & 1041.49 \\ 1054.33 & 1093.76 & 1138.50 & 1144.19 & 1147.17 & 1176.41 \\ 1181.19 & 1182.93 & 1230.30 & 1258.45 & 1260.98 & 1261.42 \\ 1328.89 & 1331.28 & 1332.85 & 1344.51 & 1365.22 & 1375.93 \\ 1387.64 & 1389.28 & 1390.85 & 1417.67 & 1470.39 & 1472.09 \\ 1473.87 & 1496.75 & 1506.71 & 1521.19 & 1556.98 & 1558.87 \\ 1579.26 & 1604.91 & 1608.43 & 1612.74 & 1620.33 & 1623.82 \\ 1657.81 & 2519.18 & 3119.66 & 3122.34 & 3122.83 & 3123.42 \\ 3152.33 & 3153.10 & 3153.23 & 3153.33 & 3171.71 & 3172.46 \\ 3172.59 & 3172.77 & 3390.33 & & & \end{array}$

TURBOMOLE Energies and Thermochemistry ${ }^{1}$ RI-PBE/TZVP,SV(P) $-5326.862913$

ZPE

RI-PBE/TZVP,SV(P) + ZPE 0.3366048

RI-PBE/TZVPP

B3-LYP/TZVP,SV(P) ZPE $(\mathrm{kJ} / \mathrm{mol})$ $-5326.526308$ $-5329.288907$ $-5328.542518$

$\ln$ (qtrans)

883.7

In(qrot)

20.39

In(qvib)

17.51

chem.pot. $(\mathrm{kJ} / \mathrm{mol})$

31.49

energy $(\mathrm{kJ} / \mathrm{mol})$

711.74

entropy $(\mathrm{kJ} / \mathrm{mol} / \mathrm{K})$

973.29

$\mathrm{Cv}(\mathrm{kJ} / \mathrm{mol}-\mathrm{K})$

0.88556

$\mathrm{Cp}((\mathrm{kJ} / \mathrm{mol}-\mathrm{K})$

0.552933

0.5612473

$\mathrm{RI}$ - PBE/TZVP, SV (P) $<\mathrm{S}^{2}>$

0.88

$\mathrm{RI}-\mathrm{PBE} / \mathrm{TZVPP}<\mathrm{S}^{2}>$

0.88

B3 - LYP/TZVP, SV (P) $\quad<\mathrm{S}^{2}>$

0.80

${ }^{1}$ Freeh output; $298.15 \mathrm{~K}, 0.1 \mathrm{MPa}$, unscaled 
4-hydroxy-2,2,3,5,6-pentachloro-3,5cyclohexadieneone

\begin{tabular}{|c|c|c|c|c|c|c|}
\hline \multicolumn{7}{|l|}{14} \\
\hline \multicolumn{7}{|c|}{ Energy $=-2678.277103354$} \\
\hline $\mathrm{C}$ & \multicolumn{2}{|c|}{-0.1469044} & \multicolumn{2}{|c|}{-1.4197071} & \multicolumn{2}{|c|}{0.2212197} \\
\hline $\mathrm{C}$ & \multicolumn{2}{|c|}{1.1590530} & \multicolumn{2}{|c|}{-0.5664346} & \multicolumn{2}{|c|}{0.0166380} \\
\hline $\mathrm{C}$ & \multicolumn{2}{|c|}{0.9776999} & \multicolumn{2}{|c|}{0.9033553} & \multicolumn{2}{|c|}{0.1193148} \\
\hline $\mathrm{C}$ & \multicolumn{2}{|c|}{-0.2566706} & \multicolumn{2}{|c|}{1.5000941} & \multicolumn{2}{|c|}{0.0944942} \\
\hline $\mathrm{C}$ & \multicolumn{2}{|c|}{-1.4751303} & \multicolumn{2}{|c|}{0.6813986} & \multicolumn{2}{|c|}{0.0541383} \\
\hline $\mathrm{C}$ & \multicolumn{2}{|c|}{-1.4288419} & \multicolumn{2}{|c|}{-0.6935113} & \multicolumn{2}{|c|}{0.0615893} \\
\hline $\mathrm{Cl}$ & \multicolumn{2}{|c|}{2.3854904} & \multicolumn{2}{|c|}{1.9300369} & \multicolumn{2}{|c|}{0.0864069} \\
\hline $\mathrm{Cl}$ & \multicolumn{2}{|c|}{-2.9691391} & \multicolumn{2}{|c|}{1.5281779} & \multicolumn{2}{|c|}{-0.0108388} \\
\hline $\mathrm{Cl}$ & \multicolumn{2}{|c|}{-2.8488488} & \multicolumn{2}{|c|}{-1.6596729} & \multicolumn{2}{|c|}{0.0438141} \\
\hline $\mathrm{Cl}$ & \multicolumn{2}{|c|}{2.4114823} & \multicolumn{2}{|c|}{-1.1532048} & \multicolumn{2}{|c|}{1.1450060} \\
\hline $\mathrm{Cl}$ & \multicolumn{2}{|c|}{1.6381127} & -0.97 & 2069 & -1.71494 & \\
\hline $\mathrm{O}$ & -0 & 0781353 & -2.60 & 1371 & 0.46319 & \\
\hline O & -0 & 4394835 & 2.83 & 6296 & 0.10269 & \\
\hline $\mathrm{H}$ & & 4625295 & 3.24 & 8565 & 0.10371 & \\
\hline & $i$ & al frequ & & & & \\
\hline & $\begin{array}{l}.00 \\
.88\end{array}$ & $\begin{array}{r}0.00 \\
64.08\end{array}$ & $\begin{array}{r}0.00 \\
98.17\end{array}$ & $\begin{array}{r}0.00 \\
122.24\end{array}$ & $\begin{array}{r}0.00 \\
158.43\end{array}$ & $\begin{array}{r}0.00 \\
177.01\end{array}$ \\
\hline & .86 & 205.80 & 219.91 & 264.22 & 287.28 & 317.24 \\
\hline & & 352.60 & 390.59 & 442.27 & 459.21 & 492.70 \\
\hline & & 627.77 & 658.01 & 679.46 & 702.04 & 764.71 \\
\hline & .70 & 885.52 & 935.34 & 1082.25 & 1169.59 & 1206.07 \\
\hline 12 & .38 & 1411.59 & 1541.35 & 1629.46 & 1757.98 & 3385.80 \\
\hline
\end{tabular}

TURBOMOLE Energies and Thermochemistry ${ }^{1}$

\begin{tabular}{lr}
\hline RI-PBE/TZVP,SV(P) & -2678.277103 \\
\hline ZPE & 0.0584296 \\
RI-PBE/TZVP,SV(P) + ZPE & -2678.218674 \\
RI-PBE/TZVPP & -2679.505804 \\
B3-LYP/TZVP,SV(P) & -2679.148442 \\
ZPE (kJ/mol) & 153.4 \\
In(qtrans) & 19.07 \\
In(qrot) & 15.11 \\
In(qvib) & 10.06 \\
chem.pot. (kJ/mol) & 43.73 \\
energy $(\mathrm{kJ} / \mathrm{mol})$ & 187.6 \\
entropy $(\mathrm{kJ} / \mathrm{mol} / \mathrm{K})$ & 0.49086 \\
CV $(\mathrm{kJ} / \mathrm{mol}-\mathrm{K})$ & 0.1881501 \\
Cp $((\mathrm{kJ} / \mathrm{mol}-\mathrm{K})$ & 0.1964644 \\
\hline
\end{tabular}

${ }^{1}$ Freeh output; 298.15K, 0.1MPa, unscaled

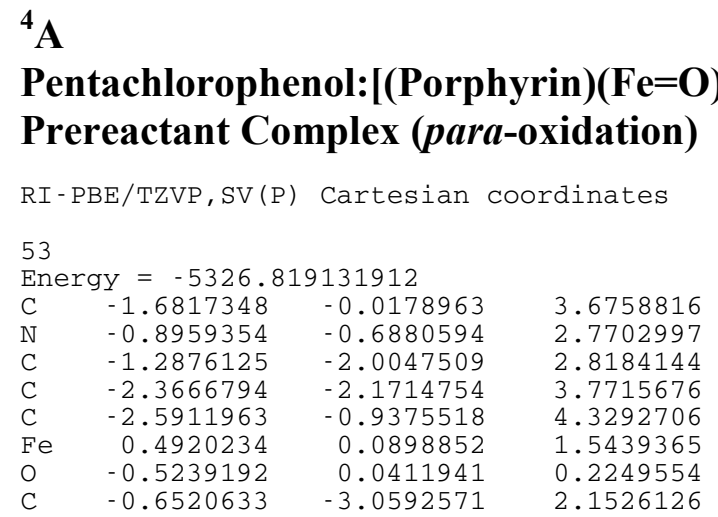




$\begin{array}{lrrr}\mathrm{C} & 0.5359279 & -2.9409133 & 1.4265735 \\ \mathrm{~N} & 1.1868115 & -1.7539993 & 1.1786618 \\ \mathrm{C} & 2.3482686 & -2.0776317 & 0.5135191 \\ \mathrm{C} & 2.4506277 & -3.5150908 & 0.3664583 \\ \mathrm{C} & 1.3120039 & -4.0509342 & 0.9138167 \\ \mathrm{C} & 3.2375751 & -1.1572431 & -0.0498417 \\ \mathrm{C} & 3.0444125 & 0.2247955 & -0.0914847 \\ \mathrm{~N} & 1.9838338 & 0.9003589 & 0.4805027 \\ \mathrm{C} & 2.0710853 & 2.2028215 & 0.0410343 \\ \mathrm{C} & 3.2263346 & 2.3630149 & -0.8164032 \\ \mathrm{C} & 3.8445790 & 1.1397830 & -0.8778375 \\ \mathrm{C} & 1.2129703 & 3.2318291 & 0.4365642 \\ \mathrm{C} & 0.2289729 & 3.1078910 & 1.4237151 \\ \mathrm{~N} & -0.1024805 & 1.9362486 & 2.0647122 \\ \mathrm{C} & -0.9962043 & 2.2777662 & 3.0538119 \\ \mathrm{C} & -1.2195057 & 3.7088989 & 3.0547670 \\ \mathrm{C} & -0.4817794 & 4.2218755 & 2.0172363 \\ \mathrm{C} & -1.6883378 & 1.3686663 & 3.8593853 \\ \mathrm{~S} & 2.1659191 & 0.0632295 & 3.3151409 \\ \mathrm{H} & 2.4270550 & 1.4097068 & 3.3451765 \\ \mathrm{H} & -0.3958938 & 5.2657015 & 1.6870825 \\ \mathrm{H} & -1.8862342 & 4.2372867 & 3.7491542 \\ \mathrm{H} & -2.3764737 & 1.7788735 & 4.6146347 \\ \mathrm{H} & -3.3218456 & -0.6549190 & 5.0989177 \\ \mathrm{H} & -2.8593744 & -3.1264876 & 3.9982037 \\ \mathrm{H} & -1.0662395 & -4.0699379 & 2.2893312 \\ \mathrm{H} & 1.0176078 & -5.1062990 & 0.9873014 \\ \mathrm{H} & 3.2857726 & -4.0342659 & -0.1221793 \\ \mathrm{H} & 4.1134697 & -1.5609877 & -0.5799340 \\ \mathrm{H} & 4.7479833 & 0.8571550 & -1.4342383 \\ \mathrm{H} & 3.5209688 & 3.3049323 & -1.2974736 \\ \mathrm{H} & 1.3824817 & 4.2313860 & 0.0081642 \\ \mathrm{C} & 0.1429154 & -0.2454143 & -3.0574792 \\ \mathrm{C} & -0.4819369 & 1.0194558 & -2.9670476 \\ \mathrm{C} & -0.5529128 & -1.3968623 & -2.6305153 \\ \mathrm{C} & -1.8603221 & -1.2675582 & -2.1321097 \\ \mathrm{C} & -1.7928182 & 1.1353442 & -2.4637779 \\ \mathrm{C} & -2.4958147 & -0.0104558 & -2.0302176 \\ \mathrm{Cl} & 0.3713414 & 2.4412846 & -3.4616971 \\ \mathrm{C} 1 & -2.5644262 & 2.6745890 & -2.3113249 \\ \mathrm{Cl} & -2.7476909 & -2.6486084 & -1.5400501 \\ \mathrm{Cl} & 0.1971615 & -2.9532286 & -2.6941751 \\ \mathrm{Cl} & 1.7555983 & -0.3845261 & -3.6733046 \\ \mathrm{O} & -3.7350164 & 0.1282467 & -1.5248643 \\ \mathrm{H} & -4.0245769 & -0.7715658 & -1.2271894\end{array}$

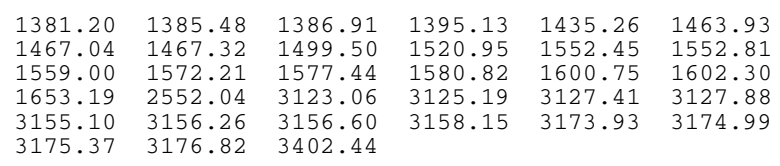

TURBOMOLE Energies and Thermochemistry ${ }^{1}$

\begin{tabular}{|c|c|}
\hline RI-PBE/TZVP,SV(P) & -5326.819132 \\
\hline $\mathrm{ZPE}$ & 0.3354266 \\
\hline RI-PBE/TZVP,SV(P) + ZPE & -5326.483705 \\
\hline RI-PBE/TZVPP & -5329.252422 \\
\hline B3-LYP/TZVP,SV(P) & -5328.49269 \\
\hline $\mathrm{ZPE}(\mathrm{kJ} / \mathrm{mol})$ & 880.7 \\
\hline In(qtrans) & 20.39 \\
\hline $\ln$ (qrot) & 17.45 \\
\hline $\ln (q v i b)$ & 37.9 \\
\hline chem.pot. (kJ/mol) & 692.92 \\
\hline energy $(\mathrm{kJ} / \mathrm{mol})$ & 974.18 \\
\hline entropy $(\mathrm{kJ} / \mathrm{mol} / \mathrm{K})$ & 0.95168 \\
\hline $\mathrm{Cv}(\mathrm{kJ} / \mathrm{mol}-\mathrm{K})$ & 0.5648757 \\
\hline $\mathrm{Cp}((\mathrm{kJ} / \mathrm{mol}-\mathrm{K})$ & 0.57319 \\
\hline $\mathrm{RI}-\mathrm{PBE} / \mathrm{TZVP}, \mathrm{SV}(\mathrm{P}) \quad\left\langle\mathrm{S}^{2}>\right.$ & 3.77 \\
\hline $\mathrm{RI}-\mathrm{PBE} / \mathrm{TZVPP}<\mathrm{S}^{2}>$ & 3.77 \\
\hline B3 - LYP / TZVP, SV (P) $<\mathrm{S}^{2}>$ & 3.79 \\
\hline
\end{tabular}

${ }^{1}$ Freeh output; $298.15 \mathrm{~K}, 0.1 \mathrm{MPa}$, unscaled 
${ }^{4} \mathrm{~A}$

\section{Pentachlorophenol: $[($ Porphyrin $)(\mathrm{Fe}=\mathbf{O})(\mathrm{SH})$ Transition State (para-oxidation)}

\begin{tabular}{|c|c|c|c|}
\hline \multicolumn{2}{|c|}{ RI - PBE/TZVP, SV (P) } & Cartesian & coordinates \\
\hline 53 & & & \\
\hline & $g y=-5326.79$ & 5626031 & \\
\hline $\mathrm{C}$ & -3.2841782 & -0.9018145 & 0.3772665 \\
\hline & -2.0352719 & -1.2603366 & 0.8245475 \\
\hline $\mathrm{C}$ & -1.9706382 & -2.6303740 & 0.7162053 \\
\hline $\mathrm{C}$ & -3.2096641 & -3.1531107 & 0.1750871 \\
\hline C & -4.0344622 & -2.0756598 & -0.0236894 \\
\hline $\mathrm{Fe}$ & -0.5633662 & -0.0182092 & 1.4407109 \\
\hline $\mathrm{O}$ & 0.0715842 & -0.0069552 & -0.1804047 \\
\hline $\mathrm{C}$ & -0.8976753 & -3.4219906 & 1.1311506 \\
\hline $\mathrm{C}$ & 0.2539884 & -2.9423758 & 1.7614237 \\
\hline $\mathrm{N}$ & 0.5341892 & -1.6187274 & 2.0050255 \\
\hline $\mathrm{C}$ & 1.7375909 & -1.5995333 & 2.6747958 \\
\hline $\mathrm{C}$ & 2.2272906 & -2.9485066 & 2.8659199 \\
\hline $\mathrm{C}$ & 1.3074633 & -3.7856958 & 2.2866981 \\
\hline $\mathrm{C}$ & 2.4241759 & -0.4512950 & 3.0753961 \\
\hline C & 2.0110087 & 0.8596725 & 2.8317697 \\
\hline $\mathrm{N}$ & 0.8529969 & 1.2248135 & 2.1816844 \\
\hline C & 0.8707718 & 2.5983312 & 2.1145277 \\
\hline $\mathrm{C}$ & 2.0753024 & 3.1186158 & 2.7286123 \\
\hline $\mathrm{C}$ & 2.7802011 & 2.0345794 & 3.1863938 \\
\hline $\mathrm{C}$ & -0.1485190 & 3.3901107 & 1.5807855 \\
\hline $\mathrm{C}$ & -1.3698782 & 2.9051952 & 1.1057183 \\
\hline $\mathrm{N}$ & -1.7282684 & 1.5768410 & 1.0398178 \\
\hline $\mathrm{C}$ & -3.0218310 & 1.5561797 & 0.5652807 \\
\hline $\mathrm{C}$ & -3.4985209 & 2.9051651 & 0.3415181 \\
\hline $\mathrm{C}$ & -2.4637242 & 3.7456875 & 0.6651118 \\
\hline $\mathrm{C}$ & -3.7625996 & 0.4070692 & 0.2807446 \\
\hline$S$ & -1.3792386 & -0.0998594 & 3.6 \\
\hline $\mathrm{H}$ & -1.6089928 & 1.24 & 3.7 \\
\hline $\mathrm{H}$ & -2.4303126 & 4.8425453 & 0.6236150 \\
\hline $\mathrm{H}$ & -4.4996807 & 3.1600276 & -0.0312057 \\
\hline $\mathrm{H}$ & -4.7883978 & 0.5460465 & -0.0943019 \\
\hline $\mathrm{H}$ & -5.0614389 & -2.0581263 & -0.4123804 \\
\hline $\mathrm{H}$ & -3.4127594 & -4.2170609 & -0.0069561 \\
\hline $\mathrm{H}$ & -0.9869164 & -4.5107526 & 0.9959421 \\
\hline $\mathrm{H}$ & 1.3201623 & -4.8819829 & 2.2226517 \\
\hline $\mathrm{H}$ & 3.1648858 & -3.2058291 & 3.3765724 \\
\hline $\mathrm{H}$ & 3.3821855 & -0.5911316 & 3.5993966 \\
\hline $\mathrm{H}$ & 3.7431847 & 2.0143409 & 3.7139754 \\
\hline $\mathrm{H}$ & 2.3284326 & 4.1846897 & 8359 \\
\hline $\mathrm{H}$ & -0.0006015 & 4.4808830 & 1.5824403 \\
\hline $\mathrm{C}$ & 1.5240230 & 0.0158545 & -1.3874573 \\
\hline $\mathrm{C}$ & 1.2124448 & 1.2595770 & -2.0640981 \\
\hline $\mathrm{C}$ & 1.2065041 & -1.2099613 & -2.0911313 \\
\hline C & 0.3685013 & -1.1678754 & -3.2053142 \\
\hline $\mathrm{C}$ & 0.3738167 & 1.2743359 & -3.1844576 \\
\hline $\mathrm{C}$ & -0.0629677 & 0.0597214 & -3.7738086 \\
\hline $\mathrm{Cl}$ & 1.7808335 & 2.7399479 & -1.3977152 \\
\hline $\mathrm{Cl}$ & -0.1259838 & 2.7572955 & -3.9200188 \\
\hline $\mathrm{Cl}$ & -0.1687755 & -2.6260873 & -4.0027640 \\
\hline $\mathrm{Cl}$ & 1.7499527 & -2.7180344 & -1.4680204 \\
\hline $\mathrm{Cl}$ & 2.9477063 & -0.0068605 & -0.3725921 \\
\hline $\mathrm{O}$ & -0.8666418 & 0.1013038 & -4.8501325 \\
\hline $\mathrm{H}$ & -1.0557336 & -0.8396463 & -5.1041059 \\
\hline
\end{tabular}

Vibrational frequencies

$\begin{array}{rrrrrr}i 499.23 & 0.00 & 0.00 & 0.00 & 0.00 & 0.00 \\ 0.00 & 6.29 & 22.71 & 29.03 & 38.48 & 45.96 \\ 50.22 & 53.27 & 72.42 & 76.03 & 83.66 & 91.51 \\ 99.86 & 102.09 & 117.97 & 127.00 & 148.00 & 151.25 \\ 193.05 & 198.89 & 200.99 & 207.59 & 208.68 & 212.53 \\ 224.48 & 232.26 & 241.27 & 251.74 & 272.33 & 273.12 \\ 279.96 & 289.11 & 295.09 & 297.40 & 317.16 & 320.21 \\ 331.64 & 337.02 & 338.06 & 349.48 & 357.95 & 358.96 \\ 361.16 & 372.17 & 409.17 & 413.04 & 418.94 & 420.16 \\ 420.71 & 421.65 & 458.07 & 458.68 & 476.72 & 537.38 \\ 548.66 & 602.99 & 624.64 & 639.83 & 652.18 & 656.06 \\ 657.10 & 672.37 & 675.16 & 679.33 & 694.40 & 694.87 \\ 695.14 & 697.52 & 717.67 & 724.72 & 734.86 & 736.34 \\ 743.73 & 763.45 & 770.17 & 770.97 & 776.60 & 781.97 \\ 784.89 & 785.26 & 799.30 & 839.55 & 847.91 & 849.37 \\ 857.20 & 863.63 & 884.81 & 889.70 & 890.86 & 892.43 \\ 970.78 & 993.65 & 1002.40 & 1003.89 & 1010.40 & 1015.57 \\ 1031.17 & 1031.74 & 1035.02 & 1040.52 & 1041.30 & 1041.83 \\ 1054.79 & 1091.03 & 1140.00 & 1147.99 & 1148.50 & 1182.68 \\ 1183.43 & 1187.67 & 1219.65 & 1261.72 & 1263.31 & 1313.47 \\ 1317.27 & 1328.76 & 1330.13 & 1339.53 & 1364.73 & 1377.44 \\ 1386.63 & 1388.02 & 1393.17 & 1396.45 & 1416.14 & 1469.04 \\ 1469.63 & 1471.14 & 1502.75 & 1504.46 & 1523.97 & 1555.33 \\ 1556.43 & 1557.26 & 1577.66 & 1596.66 & 1606.95 & 1609.16 \\ 1659.75 & 2547.49 & 3120.64 & 3122.51 & 3123.66 & 3123.78 \\ 3153.15 & 3153.48 & 3154.06 & 3154.28 & 3172.46 & 3172.67 \\ 3173.21 & 3173.41 & 3373.74 & & & \end{array}$

TURBOMOLE Energies and Thermochemistry ${ }^{1}$ RI-PBE/TZVP,SV(P) $-5326.795626$

ZPE 0.3344298

RI-PBE/TZVP,SV(P) + ZPE

$-5326.461196$

RI-PBE/TZVPP

B3-LYP/TZVP,SV(P)

$-5329.226877$

$\mathrm{ZPE}(\mathrm{kJ} / \mathrm{mol})$

$-5328.461972$

$\ln$ (qtrans)

878

In(qrot)

20.39

In(qvib)

17.42

chem.pot. $(\mathrm{kJ} / \mathrm{mol})$

34.35

energy $(\mathrm{kJ} / \mathrm{mol})$

699.18

entropy $(\mathrm{kJ} / \mathrm{mol} / \mathrm{K})$

969.62

$\mathrm{Cv}(\mathrm{kJ} / \mathrm{mol}-\mathrm{K})$

0.91536

Cp $((\mathrm{kJ} / \mathrm{mol}-\mathrm{K})$

0.5583646

0.5666789

$\mathrm{RI}-\mathrm{PBE} / \mathrm{TZVP}, \mathrm{SV}(\mathrm{P})<\mathrm{S}^{2}>$

3.77

$\left.\mathrm{RI}-\mathrm{PBE} / \mathrm{TZVPP}<\mathrm{S}^{2}\right\rangle$

3.77

B3 - LYP/TZVP, SV (P) $\left\langle\mathrm{S}^{2}>\right.$

3.80

${ }^{1}$ Freeh output; $298.15 \mathrm{~K}, 0.1 \mathrm{MPa}$, unscaled 
${ }^{4} \mathrm{~A}$

\section{Pentachlorophenol: $[($ Porphyrin $)(\mathrm{Fe}=\mathbf{O})(\mathrm{SH})$ Product Complex (para-oxidation)}

\begin{tabular}{|c|c|c|c|}
\hline \multicolumn{2}{|c|}{ RI - PBE/TZVP, SV (P) } & Cartesian & coordinates \\
\hline 53 & & & \\
\hline & $g y=-5326.8$ & 2688219 & \\
\hline $\mathrm{C}$ & -2.4567236 & 1.5637435 & 2.4230621 \\
\hline 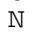 & -1.8485827 & 0.3458495 & 2.2200058 \\
\hline C & -2.8278295 & -0.6034301 & 2.4066913 \\
\hline $\mathrm{C}$ & -4.0962808 & 0.0311803 & 2.6958199 \\
\hline C & -3.8632354 & 1.3832614 & 2.7127137 \\
\hline $\mathrm{Fe}$ & 0.1141308 & 0.0126421 & 1.7798571 \\
\hline $\mathrm{O}$ & -0.4924420 & 0.0516192 & -0.1119840 \\
\hline $\mathrm{C}$ & -2.6268359 & -1.9833230 & 2.3926466 \\
\hline $\mathrm{C}$ & -1.3907211 & -2.6009627 & 2.2053452 \\
\hline $\mathrm{N}$ & -0.1938054 & -1.9595765 & 1.9432187 \\
\hline $\mathrm{C}$ & 0.7500237 & -2.9599697 & 1.7979161 \\
\hline $\mathrm{C}$ & 0.1432623 & -4.2526098 & 1.9969313 \\
\hline C & -1.1914825 & -4.0297134 & 2.2405527 \\
\hline $\mathrm{C}$ & 2.0794532 & -2.7703755 & 1.4222822 \\
\hline C & 2.6491894 & -1.5329712 & 1.1363592 \\
\hline $\mathrm{N}$ & 2.0079252 & -0.3130185 & 1.2289873 \\
\hline $\mathrm{C}$ & 2.9668497 & 0.6339833 & 0.9379232 \\
\hline $\mathrm{C}$ & 4.2225879 & -0.0023441 & 0.6071932 \\
\hline $\mathrm{C}$ & 4.0272224 & -1.3510593 & 0.7426259 \\
\hline $\mathrm{C}$ & 2.7970989 & 2.0116895 & 1.0348537 \\
\hline $\mathrm{C}$ & 1.6228646 & 2.6312141 & 1.4600599 \\
\hline $\mathrm{N}$ & 0.4461988 & 1.9841268 & 1.7910270 \\
\hline $\mathrm{C}$ & -0.4636475 & 2.9832030 & 2.0909322 \\
\hline $\mathrm{C}$ & 0.1640997 & 4.2779732 & 1.9804339 \\
\hline $\mathrm{C}$ & 1.4610155 & 4.0591358 & 1.5814653 \\
\hline $\mathrm{C}$ & -1.8144817 & 2.8007093 & 2.3842692 \\
\hline$S$ & 0.6105305 & -0.1071548 & 3.9 \\
\hline $\mathrm{H}$ & 1.0149187 & 1.1922976 & 4.13 \\
\hline $\mathrm{H}$ & 2.2483725 & 4.7943596 & 1.3689618 \\
\hline $\mathrm{H}$ & -0.3453701 & 5.2332043 & 2.1650055 \\
\hline $\mathrm{H}$ & -2.4180356 & 3.6992894 & 2.5818839 \\
\hline $\mathrm{H}$ & -4.5699501 & 2.2010088 & 2.9074200 \\
\hline $\mathrm{H}$ & -5.0358695 & -0.5075199 & 2.8773867 \\
\hline $\mathrm{H}$ & -3.4980600 & -2.6307926 & 2.5724001 \\
\hline $\mathrm{H}$ & -1.9871684 & -4.7638767 & 2.4250970 \\
\hline $\mathrm{H}$ & 0.6795481 & -5.2087652 & 1.9336605 \\
\hline $\mathrm{H}$ & 2.7152247 & -3.6626501 & 1.3232394 \\
\hline $\mathrm{H}$ & 4.7402464 & -2.1700528 & 0.5812334 \\
\hline $\mathrm{H}$ & 5.1322867 & 0.5369399 & 3483 \\
\hline $\mathrm{H}$ & 3.65 & 5539001 & 6935 \\
\hline $\mathrm{C}$ & -0.3965603 & 0.0337509 & -1.3852779 \\
\hline C & -0.4265873 & 1.2598094 & -2.1761030 \\
\hline $\mathrm{C}$ & -0.4706437 & -1.2093374 & -2.1491689 \\
\hline C & -0.6529155 & -1.1932956 & -3.5334601 \\
\hline $\mathrm{C}$ & -0.5938484 & 1.2472006 & -3.5633526 \\
\hline $\mathrm{C}$ & -0.6979565 & 0.0177322 & -4.2665203 \\
\hline $\mathrm{Cl}$ & -0.3483364 & 2.7561387 & -1.3341320 \\
\hline $\mathrm{Cl}$ & -0.6564281 & 2.7115556 & -4.4814580 \\
\hline $\mathrm{Cl}$ & -0.7742370 & -2.6702899 & -4.4590239 \\
\hline $\mathrm{Cl}$ & -0.4848319 & -2.6962033 & -1.2914145 \\
\hline $\mathrm{Cl}$ & 2.2959294 & -0.2501357 & -2.1398635 \\
\hline $\mathrm{O}$ & -0.8375725 & 0.0358397 & -5.6014067 \\
\hline $\mathrm{H}$ & -0.8718591 & -0.9113592 & -5.8983471 \\
\hline
\end{tabular}

$\begin{array}{rrrrrr}143.06 & 0.00 & 0.00 & 0.00 & 0.00 & 0.00 \\ 0.00 & 8.77 & 28.58 & 38.90 & 43.76 & 51.95 \\ 54.05 & 56.43 & 63.11 & 72.67 & 78.66 & 87.77 \\ 102.72 & 105.96 & 118.58 & 120.33 & 126.91 & 142.64 \\ 148.96 & 171.98 & 196.38 & 200.41 & 207.58 & 210.32 \\ 218.35 & 228.93 & 248.00 & 257.08 & 266.99 & 269.39 \\ 272.47 & 276.98 & 281.18 & 283.71 & 301.91 & 322.92 \\ 328.89 & 335.56 & 339.26 & 354.35 & 358.52 & 360.03 \\ 361.52 & 370.71 & 399.76 & 403.26 & 413.03 & 415.59 \\ 422.76 & 423.61 & 452.35 & 453.27 & 503.96 & 523.54 \\ 549.98 & 578.43 & 621.38 & 644.01 & 651.00 & 653.25 \\ 659.88 & 669.75 & 673.29 & 680.70 & 691.52 & 694.03 \\ 694.52 & 704.04 & 717.44 & 723.42 & 730.98 & 735.51 \\ 760.35 & 769.30 & 772.90 & 779.92 & 780.01 & 783.12 \\ 786.70 & 797.86 & 833.43 & 842.60 & 844.12 & 851.54 \\ 859.81 & 888.67 & 892.47 & 895.15 & 895.85 & 905.39 \\ 992.56 & 995.23 & 999.97 & 1013.21 & 1017.51 & 1029.55 \\ 1029.82 & 1032.15 & 1036.65 & 1041.71 & 1042.16 & 1042.64 \\ 1053.00 & 1138.61 & 1142.17 & 1145.19 & 1157.33 & 1182.24 \\ 1183.58 & 1209.42 & 1260.32 & 1261.79 & 1291.05 & 1319.66 \\ 1331.96 & 1332.92 & 1348.80 & 1363.64 & 1366.17 & 1372.30 \\ 1378.57 & 1386.75 & 1393.83 & 1407.70 & 1445.00 & 1464.97 \\ 1470.49 & 1478.46 & 1501.20 & 1512.46 & 1519.49 & 1549.37 \\ 1556.85 & 1558.90 & 1580.28 & 1596.60 & 1618.39 & 1626.78 \\ 1666.57 & 2541.51 & 3127.63 & 3128.48 & 3130.66 & 3133.14 \\ 3155.40 & 3156.33 & 3156.88 & 3161.64 & 3174.29 & 3174.83 \\ 3175.51 & 3180.75 & 3367.94 & & & \end{array}$

TURBOMOLE Energies and Thermochemistry

\begin{tabular}{lr}
\hline RI-PBE/TZVP,SV(P) & -5326.842688 \\
\hline ZPE & 0.3351379 \\
RI-PBE/TZVP,SV(P) + ZPE & -5326.50755 \\
RI-PBE/TZVPP & -5329.271462 \\
B3-LYP/TZVP,SV(P) & -5328.520775 \\
ZPE (kJ/mol) & 879.9 \\
In(qtrans) & 20.39 \\
In(qrot) & 17.5 \\
In(qvib) & 33.98 \\
chem.pot. $(\mathrm{kJ} / \mathrm{mol})$ & 701.75 \\
energy $(\mathrm{kJ} / \mathrm{mol})$ & 972.05 \\
entropy $(\mathrm{kJ} / \mathrm{mol} / \mathrm{K})$ & 0.9149 \\
CV $(\mathrm{kJ} / \mathrm{mol}-\mathrm{K})$ & 0.5581183 \\
Cp $((\mathrm{kJ} / \mathrm{mol}-\mathrm{K})$ & 0.5664326 \\
$\mathrm{RI}-\mathrm{PBE} / \mathrm{TZVP}, \mathrm{SV}(\mathrm{P}) \quad \mathrm{SS}^{2}>$ & 3.79 \\
$\mathrm{RI}-\mathrm{PBE} / \mathrm{TZVPP}<\mathrm{S}^{2}>$ & 3.79 \\
$\mathrm{~B} 3-\mathrm{LYP} / \mathrm{TZVP}, \mathrm{SV}(\mathrm{P}) \quad<\mathrm{S}^{2}>$ & 3.94 \\
\hline
\end{tabular}

${ }^{1}$ Freeh output; $298.15 \mathrm{~K}, 0.1 \mathrm{MPa}$, unscaled 
${ }^{2} \mathbf{A}$

\section{Pentachlorophenol: $[($ Porphyrin $)(\mathrm{Fe}=\mathbf{O})(\mathrm{SH})$ Prereactant Complex (meta-oxidation)}

RI-PBE/TZVP, SV (P) Cartesian coordinates

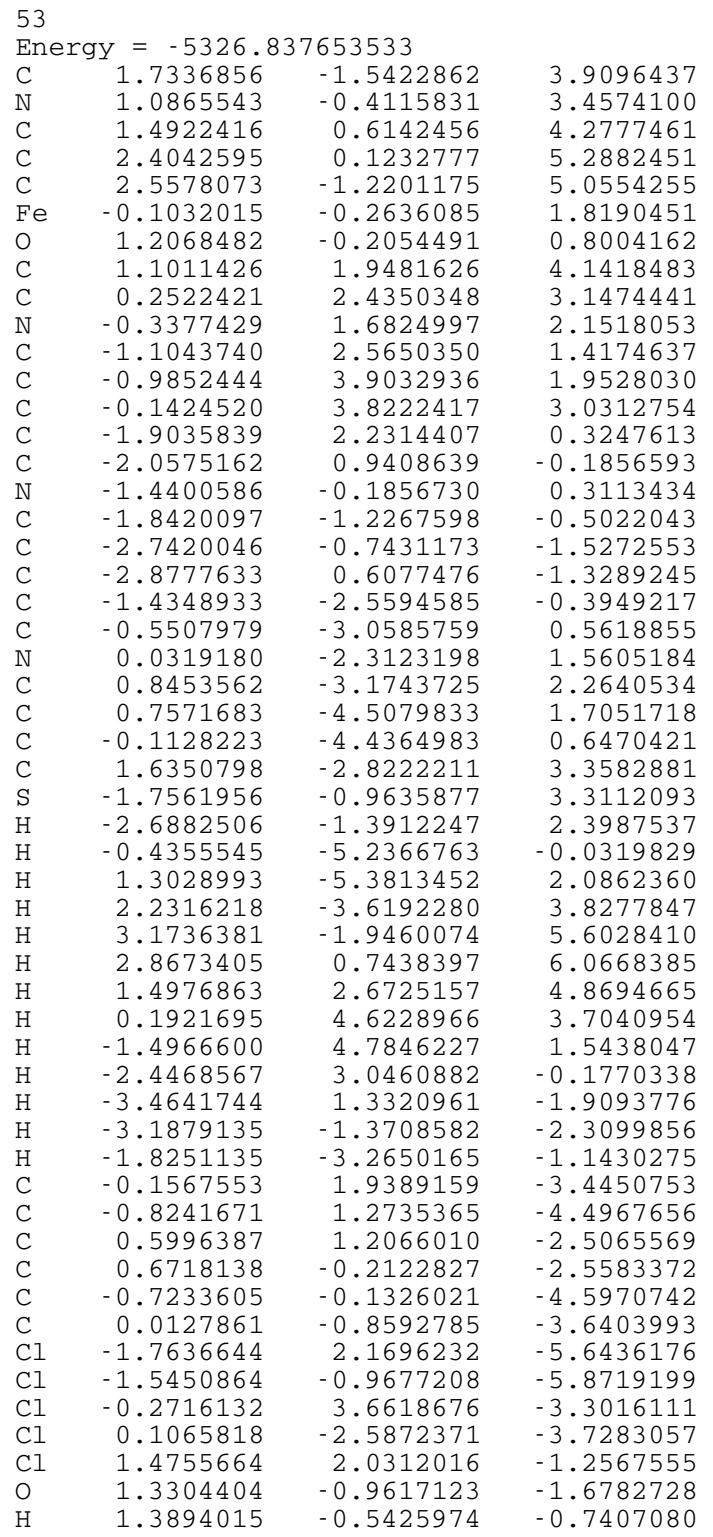

Vibrational frequencies

$\begin{array}{rrrrrr}0.00 & 0.00 & 0.00 & 0.00 & 0.00 & 0.00 \\ 5.28 & 12.39 & 22.50 & 35.37 & 43.51 & 50.83 \\ 54.17 & 63.69 & 78.42 & 84.74 & 94.93 & 101.96 \\ 106.51 & 129.95 & 147.73 & 148.13 & 175.94 & 187.29 \\ 198.23 & 203.74 & 205.73 & 206.85 & 208.67 & 216.65 \\ 225.59 & 239.37 & 242.17 & 270.03 & 274.29 & 278.18 \\ 280.85 & 293.79 & 298.76 & 305.78 & 315.92 & 323.26 \\ 331.45 & 337.69 & 344.82 & 351.68 & 359.22 & 360.23 \\ 368.29 & 372.08 & 397.23 & 404.77 & 416.56 & 417.72 \\ 420.24 & 422.12 & 456.40 & 457.70 & 542.10 & 555.83 \\ 630.15 & 633.17 & 637.29 & 648.87 & 655.38 & 655.55 \\ 671.02 & 674.41 & 690.69 & 693.24 & 697.80 & 705.28 \\ 712.96 & 723.43 & 733.27 & 734.51 & 738.74 & 759.39 \\ 763.46 & 771.87 & 773.75 & 779.71 & 780.22 & 784.73 \\ 784.95 & 798.48 & 808.19 & 838.94 & 849.15 & 850.97 \\ 859.74 & 864.89 & 889.73 & 894.31 & 895.50 & 896.91 \\ 978.17 & 993.36 & 1001.94 & 1002.73 & 1011.56 & 1015.70 \\ 1033.15 & 1033.54 & 1037.67 & 1041.89 & 1043.26 & 1044.60 \\ 1053.60 & 1115.18 & 1137.37 & 1147.36 & 1147.53 & 1182.37 \\ 1184.48 & 1238.11 & 1262.03 & 1263.42 & 1276.39 & 1299.47 \\ 1328.71 & 1330.49 & 1340.96 & 1342.06 & 1363.16 & 1367.17 \\ 1375.52 & 1378.30 & 1388.00 & 1388.77 & 1444.94 & 1467.41 \\ 1471.12 & 1472.34 & 1503.77 & 1521.26 & 1524.62 & 1555.58 \\ 1557.32 & 1561.70 & 1577.49 & 1593.23 & 1608.67 & 1609.96 \\ 1663.58 & 2547.64 & 2689.66 & 3125.01 & 3126.54 & 3131.18 \\ 3135.58 & 3157.21 & 3158.16 & 3158.31 & 3159.44 & 3175.87 \\ 3176.86 & 3176.96 & 3177.72 & & & \end{array}$

TURBOMOLE Energies and Thermochemistry ${ }^{1}$ RI-PBE/TZVP,SV(P) $-5326.837654$

ZPE 0.3351302

RI-PBE/TZVP,SV(P) + ZPE

RI-PBE/TZVPP

B3-LYP/TZVP,SV(P)

$-5326.502523$

$\mathrm{ZPE}(\mathrm{kJ} / \mathrm{mol})$

$-5329.266885$

$\ln$ (qtrans)

$-5328.504792$

In(qrot)

879.9

In(qvib)

20.39

17.62

energy $(\mathrm{kJ} / \mathrm{mol})$

697.87

entropy $(\mathrm{kJ} / \mathrm{mol} / \mathrm{K})$

971.96

$\mathrm{Cv}(\mathrm{kJ} / \mathrm{mol}-\mathrm{K})$

0.92763

Cp $((\mathrm{kJ} / \mathrm{mol}-\mathrm{K})$

0.5611705

0.5694848

$\mathrm{RI}-\mathrm{PBE} / \mathrm{TZVP}, \mathrm{SV}(\mathrm{P}) \quad<\mathrm{S}^{2}>$

1.18

$\mathrm{RI}-\mathrm{PBE} / \mathrm{TZVPP}<\mathrm{S}^{2}>$

1.14

B3 - LYP / TZVP , SV (P) $<\mathrm{S}^{2}>$

1.66

${ }^{1}$ Freeh output; $298.15 \mathrm{~K}, 0.1 \mathrm{MPa}$, unscaled 
${ }^{2} \mathbf{A}$

\section{Pentachlorophenol:[(Porphyrin $)(\mathrm{Fe}=\mathbf{O})(\mathrm{SH})]$ Transition State (meta-oxidation)}

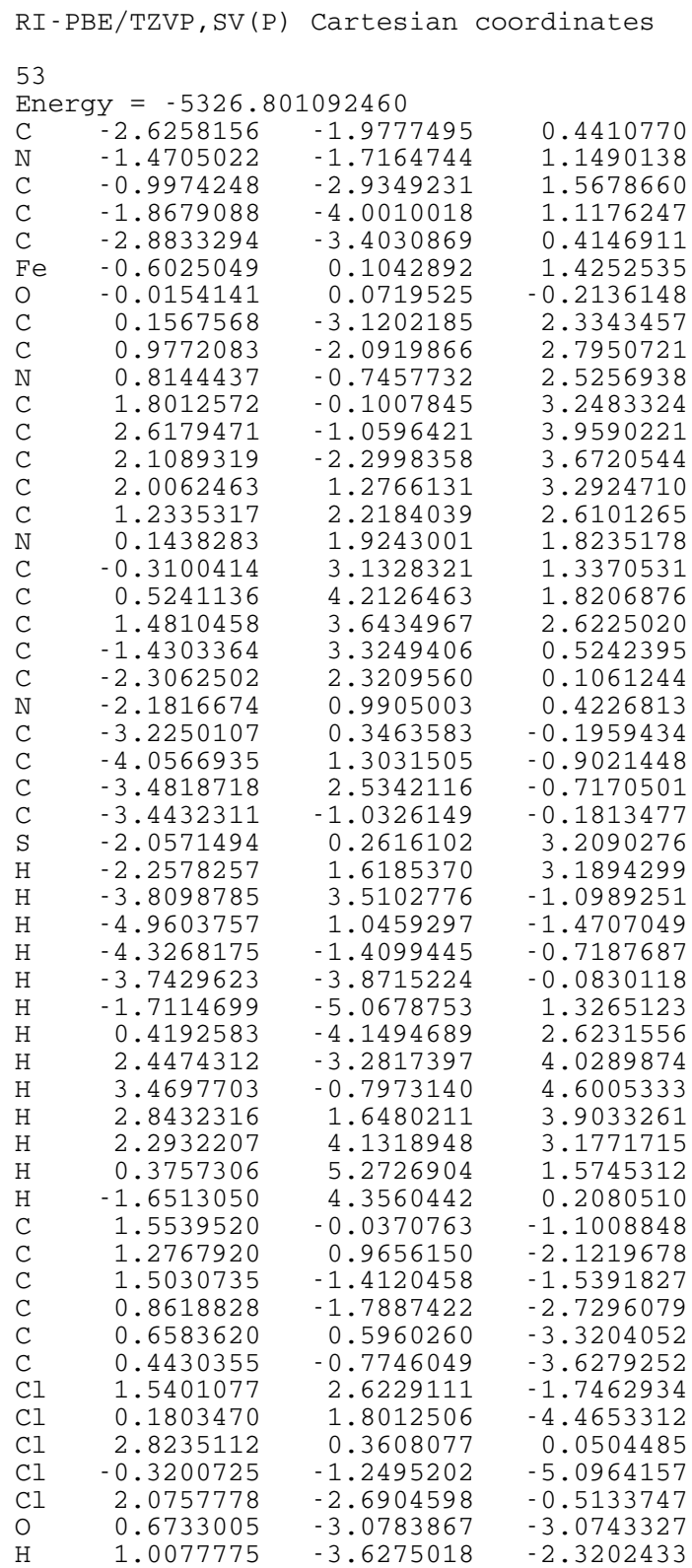

$\begin{array}{rrrrrr}\text { i538.66 } & 0.00 & 0.00 & 0.00 & 0.00 & 0.00 \\ 0.00 & 12.56 & 23.24 & 28.78 & 44.48 & 47.50 \\ 54.04 & 58.71 & 74.93 & 77.93 & 99.08 & 101.66 \\ 105.54 & 108.27 & 121.30 & 139.38 & 149.74 & 157.97 \\ 177.04 & 197.36 & 200.00 & 206.49 & 209.41 & 213.46 \\ 222.40 & 232.57 & 253.35 & 255.32 & 262.93 & 272.75 \\ 276.93 & 286.18 & 295.90 & 299.86 & 307.81 & 322.09 \\ 326.40 & 349.27 & 353.78 & 355.94 & 361.22 & 367.02 \\ 369.47 & 378.89 & 394.37 & 406.06 & 417.51 & 419.75 \\ 421.21 & 422.52 & 448.63 & 458.78 & 481.40 & 508.60 \\ 578.16 & 628.60 & 643.94 & 646.58 & 652.80 & 657.15 \\ 657.83 & 673.11 & 679.53 & 686.84 & 694.38 & 695.24 \\ 699.05 & 707.90 & 717.09 & 724.23 & 734.92 & 735.64 \\ 762.15 & 764.52 & 771.78 & 772.05 & 777.89 & 782.52 \\ 784.95 & 785.28 & 798.74 & 841.45 & 849.87 & 851.59 \\ 852.30 & 858.91 & 885.94 & 891.39 & 891.66 & 893.68 \\ 961.21 & 991.11 & 1000.08 & 1001.93 & 1010.25 & 1013.58 \\ 1030.72 & 1031.96 & 1035.43 & 1040.42 & 1041.09 & 1041.66 \\ 1053.52 & 1114.37 & 1139.55 & 1148.18 & 1150.02 & 1182.94 \\ 1183.59 & 1186.14 & 1231.63 & 1261.23 & 1263.43 & 1306.30 \\ 1319.44 & 1328.45 & 1332.25 & 1342.09 & 1363.43 & 1367.46 \\ 1374.00 & 1383.09 & 1387.11 & 1390.83 & 1393.39 & 1470.09 \\ 1470.55 & 1473.39 & 1504.95 & 1519.68 & 1524.17 & 1542.52 \\ 1556.87 & 1558.85 & 1579.47 & 1605.63 & 1612.66 & 1614.36 \\ 1662.62 & 2548.09 & 3122.13 & 3122.37 & 3122.96 & 3123.65 \\ 3153.54 & 3154.09 & 3154.26 & 3154.42 & 3172.71 & 3173.29 \\ 3173.44 & 3173.59 & 3403.16 & & & \end{array}$

TURBOMOLE Energies and Thermochemistry ${ }^{1}$

\begin{tabular}{lr}
\hline RI-PBE/TZVP,SV(P) & -5326.801092 \\
\hline ZPE & 0.3349289 \\
RI-PBE/TZVP,SV(P) + ZPE & -5326.466164 \\
RI-PBE/TZVPP & -5329.231333 \\
B3-LYP/TZVP,SV(P) & -5328.460617 \\
ZPE (kJ/mol) & 879.3 \\
In(qtrans) & 20.39 \\
In(qrot) & 17.41 \\
In(qvib) & 32.88 \\
chem.pot. $(\mathrm{kJ} / \mathrm{mol})$ & 704.15 \\
energy $(\mathrm{kJ} / \mathrm{mol})$ & 970.36 \\
entropy $(\mathrm{kJ} / \mathrm{mol} / \mathrm{K})$ & 0.90118 \\
CV $(\mathrm{kJ} / \mathrm{mol}-\mathrm{K})$ & 0.5572147 \\
Cp $((\mathrm{kJ} / \mathrm{mol}-\mathrm{K})$ & 0.565529 \\
$\mathrm{RI}-\mathrm{PBE} / \mathrm{TZVP}, \mathrm{SV}(\mathrm{P}) \quad<\mathrm{S}^{2}>$ & 0.95 \\
$\mathrm{RI}-\mathrm{PBE} / \mathrm{TZVPP}<\mathrm{S}^{2}>$ & 0.93 \\
$\mathrm{~B} 3-\mathrm{LYP} / \mathrm{TZVP}, \mathrm{SV}(\mathrm{P}) \quad \mathrm{S}^{2}>$ & 1.53 \\
\hline
\end{tabular}

${ }^{1}$ Freeh output; 298.15K, 0.1MPa, unscaled 
${ }^{2} \mathbf{A}$

\section{Pentachlorophenol: [(Porphyrin $)(\mathrm{Fe}=\mathbf{O})(\mathrm{SH})]$ Product Complex (meta-oxidation)}

\begin{tabular}{|c|c|c|c|}
\hline \multicolumn{2}{|c|}{ RI - PBE/TZVP , SV (P) } & Cartesian & coordinates \\
\hline & & & \\
\hline En & $y=-5326.86$ & 7528017 & \\
\hline $\mathrm{C}$ & -2.8375170 & -0.9001851 & 2.0970344 \\
\hline $\mathrm{N}$ & -1.4806127 & -1.1287034 & 2.1936717 \\
\hline $\mathrm{C}$ & -1.3495980 & -2.4432599 & 2.5899540 \\
\hline $\mathrm{C}$ & -2.6518458 & -3.0585505 & 2.7426589 \\
\hline C & -3.5796150 & -2.1002464 & 2.4231983 \\
\hline $\mathrm{Fe}$ & 0.0175956 & 0.1866454 & 1.8989945 \\
\hline 0 & -0.0389484 & -0.2405104 & -0.0982068 \\
\hline C & -0.1414235 & -3.1171741 & 2.7750681 \\
\hline $\mathrm{C}$ & 1.1230041 & -2.5748712 & 2.5366932 \\
\hline $\mathrm{N}$ & 1.3775786 & -1.2797612 & 2.1360859 \\
\hline $\mathrm{C}$ & 2.7471201 & -1.1931441 & 1.9929898 \\
\hline $\mathrm{C}$ & 3.3695491 & -2.4665324 & 2.2869185 \\
\hline C & 2.3572564 & -3.3243141 & 2.6374707 \\
\hline $\mathrm{C}$ & 3.4500084 & -0.0318401 & 1.6665357 \\
\hline C & 2.8635493 & 1.2220080 & 1.4868965 \\
\hline $\mathrm{N}$ & 1.5105512 & 1.4851836 & 1.5501755 \\
\hline $\mathrm{C}$ & 1.3831022 & 2.8439674 & 1.3508752 \\
\hline $\mathrm{C}$ & 2.6840174 & 3.4519935 & 1.1622971 \\
\hline C & 3.6064306 & 2.4394159 & 1.2367208 \\
\hline $\mathrm{C}$ & 0.1780600 & 3.5448233 & 1.2840733 \\
\hline $\mathrm{C}$ & -1.0896753 & 2.9688330 & 1.3733446 \\
\hline $\mathrm{N}$ & -1.3494232 & 1.6314588 & 1.5811690 \\
\hline $\mathrm{C}$ & -2.7221407 & 1.5073757 & 1.5598008 \\
\hline $\mathrm{C}$ & -3.3445997 & 2.7929045 & 1.3199157 \\
\hline $\mathrm{C}$ & -2.3265062 & 3.7058211 & 1.213 \\
\hline C & -3.4279002 & 0.3227823 & 1.7 \\
\hline S & 0.04 & 0.6394228 & 4.0330943 \\
\hline $\mathrm{H}$ & 1.3992615 & 0.7312801 & 4. 2261907 \\
\hline $\mathrm{H}$ & -2.3891446 & 4.7878659 & 1.0360178 \\
\hline $\mathrm{H}$ & -4.4282913 & 2.9597325 & 1.2556104 \\
\hline $\mathrm{H}$ & -4.5268882 & 0.3661622 & 1.7323486 \\
\hline $\mathrm{H}$ & -4.6751434 & -2.1778986 & 2.4154557 \\
\hline $\mathrm{H}$ & -2.8176911 & -4.1002163 & 3.0493025 \\
\hline $\mathrm{H}$ & -0.1904549 & -4.1689192 & 3.0964735 \\
\hline $\mathrm{H}$ & 2.4233485 & -4.3794353 & 2.9357550 \\
\hline $\mathrm{H}$ & 4.4496448 & -2.6598329 & 377251 \\
\hline $\mathrm{H}$ & 4.5457871 & -0.1015812 & 30224 \\
\hline $\mathrm{H}$ & 4.699 & 2.4950587 & 1.1397286 \\
\hline $\mathrm{H}$ & 2.85 & 4.52 & 0.9850324 \\
\hline $\mathrm{H}$ & 0.23 & 4.6311215 & 1.1145378 \\
\hline C & -0.0315355 & -0.4651360 & -1.3154941 \\
\hline $\mathrm{C}$ & -0.0181629 & 0.7302696 & -2.3160409 \\
\hline C & -0.0367914 & -1.7793611 & -1.8922606 \\
\hline $\mathrm{C}$ & -0.0460113 & -2.0225112 & -3.2627817 \\
\hline C & -0.0353766 & 0.3831131 & -3.7818021 \\
\hline $\mathrm{C}$ & -0.0444237 & -0.9177159 & -4.2132194 \\
\hline $\mathrm{Cl}$ & -1.4754768 & 1.7390868 & -1.9487562 \\
\hline $\mathrm{Cl}$ & -0.0324007 & 1.6953742 & -4.8925238 \\
\hline $\mathrm{Cl}$ & 1.4958411 & 1.6709549 & -1.9781552 \\
\hline $\mathrm{Cl}$ & -0.0567653 & -1.3240442 & -5.8900377 \\
\hline $\mathrm{Cl}$ & -0.0440246 & -3.1638601 & -0.8327486 \\
\hline 0 & -0.0588298 & -3.2578297 & -3.7687604 \\
\hline $\mathrm{H}$ & -0.0586071 & -3.8818689 & -2.9914822 \\
\hline
\end{tabular}

$\begin{array}{rrrrrr}0.00 & 0.00 & 0.00 & 0.00 & 0.00 & 0.00 \\ 1.75 & 20.48 & 23.27 & 29.48 & 50.45 & 51.37 \\ 52.04 & 57.08 & 68.35 & 96.91 & 100.15 & 101.35 \\ 110.71 & 119.07 & 146.86 & 149.52 & 152.31 & 169.66 \\ 175.88 & 195.43 & 204.05 & 210.61 & 218.84 & 222.39 \\ 232.31 & 258.37 & 261.32 & 264.27 & 274.35 & 281.17 \\ 284.19 & 291.94 & 295.64 & 308.10 & 311.90 & 340.06 \\ 345.21 & 354.90 & 358.08 & 359.97 & 360.72 & 367.12 \\ 380.38 & 391.95 & 400.84 & 411.89 & 420.48 & 420.84 \\ 421.90 & 422.46 & 460.95 & 520.41 & 545.05 & 598.40 \\ 631.50 & 652.75 & 653.77 & 657.74 & 658.43 & 669.57 \\ 674.00 & 685.95 & 691.70 & 696.97 & 697.62 & 697.93 \\ 713.77 & 720.67 & 722.59 & 730.61 & 735.14 & 736.53 \\ 743.84 & 761.73 & 769.22 & 769.33 & 776.11 & 784.71 \\ 785.93 & 786.41 & 799.75 & 834.32 & 842.35 & 842.88 \\ 850.03 & 883.26 & 883.52 & 888.16 & 888.37 & 890.56 \\ 974.61 & 992.02 & 998.62 & 1003.07 & 1008.22 & 1009.70 \\ 1029.19 & 1030.61 & 1033.42 & 1039.19 & 1040.35 & 1040.82 \\ 1054.13 & 1085.07 & 1139.29 & 1145.01 & 1147.94 & 1154.38 \\ 1182.02 & 1182.76 & 1249.05 & 1261.21 & 1261.49 & 1283.33 \\ 1328.78 & 1331.56 & 1332.51 & 1343.96 & 1365.07 & 1375.49 \\ 1388.36 & 1389.78 & 1391.10 & 1436.46 & 1470.20 & 1472.65 \\ 1474.05 & 1506.46 & 1518.53 & 1521.12 & 1557.26 & 1559.64 \\ 1579.69 & 1596.76 & 1610.26 & 1614.46 & 1621.46 & 1633.69 \\ 1657.90 & 2508.79 & 3120.00 & 3121.43 & 3121.90 & 3122.64 \\ 3150.52 & 3150.79 & 3151.46 & 3151.64 & 3170.04 & 3170.41 \\ 3170.89 & 3171.11 & 3315.31 & & & \end{array}$

TURBOMOLE Energies and Thermochemistry ${ }^{1}$

\begin{tabular}{lr}
\hline RI-PBE/TZVP,SV(P) & -5326.867528 \\
\hline ZPE & 0.3369423 \\
RI-PBE/TZVP,SV(P) + ZPE & -5326.530586 \\
RI-PBE/TZVPP & -5329.293998 \\
B3-LYP/TZVP,SV(P) & -5328.549837 \\
ZPE (kJ/mol) & 884.6 \\
In(qtrans) & 20.39 \\
In(qrot) & 17.53 \\
In(qvib) & 36.1 \\
chem.pot. $(\mathrm{kJ} / \mathrm{mol})$ & 701.15 \\
energy $(\mathrm{kJ} / \mathrm{mol})$ & 976.3 \\
entropy $(\mathrm{kJ} / \mathrm{mol} / \mathrm{K})$ & 0.93115 \\
Cv $(\mathrm{kJ} / \mathrm{mol}-\mathrm{K})$ & 0.5596789 \\
Cp $((\mathrm{kJ} / \mathrm{mol}-\mathrm{K})$ & 0.5679932 \\
$\mathrm{RI}-\mathrm{PBE} / \mathrm{TZVP}, \mathrm{SV}(\mathrm{P}) \quad\left\langle\mathrm{S}^{2}\right\rangle$ & 0.85 \\
$\left.\mathrm{RI}-\mathrm{PBE} / \mathrm{TZVPP}<\mathrm{S}^{2}\right\rangle$ & 0.84 \\
$\left.\mathrm{~B} 3-\mathrm{LYP} / \mathrm{TZVP}, \mathrm{SV}(\mathrm{P}) \quad \mathrm{S}^{2}\right\rangle$ & 0.79 \\
\hline
\end{tabular}

${ }^{1}$ Freeh output; $298.15 \mathrm{~K}, 0.1 \mathrm{MPa}$, unscaled 
5-Hydroxy-2,3,3,4,6-pentachloro-3,5cyclohexadieneone

$\begin{array}{lrrr}\text { Energy }=-2678.276789187 & \\ \text { Ener } & \\ \text { C } & -0.7879760 & -1.2264742 & -0.0940605 \\ \text { C } & 0.6606252 & -1.3649780 & -0.2896918 \\ \text { C } & 1.4789981 & -0.0603890 & 0.0016549 \\ \text { C } & 0.7512773 & 1.2511653 & -0.1377674 \\ \text { C } & -0.6199330 & 1.2642915 & -0.0981137 \\ \text { C } & -1.3849992 & 0.0205249 & -0.0375940 \\ \text { O } & 1.2331004 & -2.3943396 & -0.5947315 \\ \text { Cl } & -1.4423746 & 2.7853288 & -0.1379204 \\ \text { Cl } & -3.0959384 & 0.1458045 & 0.0843899 \\ \text { Cl } & -1.6981701 & -2.6897046 & -0.0959681 \\ \text { Cl } & 1.9559291 & -0.2279389 & 1.7576997 \\ \text { Cl } & 2.9579300 & -0.0273541 & -1.0204373 \\ \text { O } & 1.4722269 & 2.3786300 & -0.1911885 \\ \text { H } & 2.4111549 & 2.1176034 & -0.3864878\end{array}$

Vibrational frequencies

$\begin{array}{rrrrrr}0.00 & 0.00 & 0.00 & 0.00 & 0.00 & 0.00 \\ 39.45 & 68.92 & 78.79 & 148.74 & 157.73 & 183.48 \\ 203.91 & 206.83 & 220.58 & 266.25 & 291.11 & 312.70 \\ 341.66 & 348.27 & 366.60 & 370.93 & 493.37 & 510.70 \\ 546.99 & 606.74 & 667.31 & 687.65 & 705.90 & 768.91 \\ 781.77 & 899.01 & 998.98 & 1044.00 & 1166.47 & 1226.84 \\ 1301.30 & 1356.41 & 1510.74 & 1642.71 & 1749.24 & 3363.68\end{array}$

TURBOMOLE Energies and Thermochemistry ${ }^{1}$

\begin{tabular}{lr}
\hline RI-PBE/TZVP,SV(P) & -2678.276789 \\
\hline ZPE & 0.0584 \\
RI-PBE/TZVP,SV(P) + ZPE & -2678.218389 \\
RI-PBE/TZVPP & -2679.504959 \\
B3-LYP/TZVP,SV(P) & -2679.147134 \\
ZPE (kJ/mol) & 153.3 \\
In(qtrans) & 19.07 \\
In(qrot) & 15.12 \\
In(qvib) & 9.51 \\
chem.pot. (kJ/mol) & 45.01 \\
energy $(\mathrm{kJ} / \mathrm{mol})$ & 187.4 \\
entropy $(\mathrm{kJ} / \mathrm{mol} / \mathrm{K})$ & 0.48589 \\
CV $(\mathrm{kJ} / \mathrm{mol}-\mathrm{K})$ & 0.1881789 \\
Cp $((\mathrm{kJ} / \mathrm{mol}-\mathrm{K})$ & 0.1964932 \\
\hline
\end{tabular}

${ }^{1}$ Freeh output; $298.15 \mathrm{~K}, 0.1 \mathrm{MPa}$, unscaled 
${ }^{4} \mathrm{~A}$

\section{Pentachlorophenol:[(Porphyrin $)(\mathrm{Fe}=\mathbf{O})(\mathrm{SH})]$ Prereactant Complex (meta-oxidation)}

\begin{tabular}{|c|c|c|c|}
\hline \multicolumn{2}{|c|}{ RI - PBE/TZVP, SV (P) } & Cartesian & coordinates \\
\hline & & & \\
\hline & $y=-5326.81$ & 8539833 & \\
\hline $\mathrm{C}$ & -1.9569895 & -1.1767941 & 3.2815089 \\
\hline 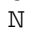 & -0.7066248 & -1.0479050 & 2.7274670 \\
\hline C & -0.0089898 & -2.1774122 & 3.0828281 \\
\hline $\mathrm{C}$ & -0.8479806 & -3.0572098 & 3.8724015 \\
\hline C & -2.0534537 & -2.4189263 & 4.0213827 \\
\hline $\mathrm{Fe}$ & -0.0306829 & 0.4482042 & 1.5687989 \\
\hline $\mathrm{O}$ & -0.2170321 & -0.4290932 & 0.1644421 \\
\hline $\mathrm{C}$ & 1.3523943 & -2.3938873 & 2.8411533 \\
\hline $\mathrm{C}$ & 2.2244561 & -1.4484080 & 2.2941848 \\
\hline $\mathrm{N}$ & 1.8451866 & -0.2087513 & 1.8325492 \\
\hline $\mathrm{C}$ & 3.0027227 & 0.4457813 & 1.4751882 \\
\hline $\mathrm{C}$ & 4.1503892 & -0.3961750 & 1.7424368 \\
\hline $\mathrm{C}$ & 3.6651275 & -1.5836875 & 2.2310824 \\
\hline $\mathrm{C}$ & 3.0633148 & 1.6939827 & 0.8471145 \\
\hline C & 1.9591113 & 2.4291207 & 0.4129340 \\
\hline $\mathrm{N}$ & 0.6378244 & 2.0645000 & 0.5903595 \\
\hline $\mathrm{C}$ & -0.1113435 & 2.9499867 & -0.1519103 \\
\hline $\mathrm{C}$ & 0.7529997 & 3.9129144 & -0.8011613 \\
\hline $\mathrm{C}$ & 2.0367935 & 3.6047825 & -0.4282462 \\
\hline $\mathrm{C}$ & -1.5075739 & 2.9632605 & -0.1975989 \\
\hline $\mathrm{C}$ & -2.3334892 & 2.1693936 & 0.6053441 \\
\hline $\mathrm{N}$ & -1.8928326 & 1.1947273 & 1.4712838 \\
\hline $\mathrm{C}$ & -2.9996373 & 0.7744099 & 2.1724523 \\
\hline $\mathrm{C}$ & -4.1703778 & 1.5176974 & 1.7540634 \\
\hline $\mathrm{C}$ & -3.7614459 & 2.3634525 & 0.7535807 \\
\hline $\mathrm{C}$ & -3.0209790 & -0.2937753 & 3.0737877 \\
\hline S & 0.4810745 & 1.8080487 & 3.52 \\
\hline $\mathrm{H}$ & -0.3370305 & 2.8717311 & 0411 \\
\hline $\mathrm{H}$ & -4.3577514 & 3.0829670 & 0.1768586 \\
\hline $\mathrm{H}$ & -5.17 & 1.3779625 & 2.1641357 \\
\hline $\mathrm{H}$ & -3.9767455 & -0.5221517 & 3.5701813 \\
\hline $\mathrm{H}$ & -2.9471293 & -2.7575788 & 4.5623024 \\
\hline $\mathrm{H}$ & -0.5298717 & -4.0265192 & 4.2788828 \\
\hline $\mathrm{H}$ & 1.7856375 & -3.3420720 & 3.1950827 \\
\hline $\mathrm{H}$ & 4.2248315 & -2.4729511 & 2.5507664 \\
\hline $\mathrm{H}$ & 5.1930797 & -0.1068070 & 1.5555669 \\
\hline $\mathrm{H}$ & 4.0610447 & 2.0891344 & 0.6029484 \\
\hline $\mathrm{H}$ & 2.9717301 & 4.1017359 & -0.7190997 \\
\hline $\mathrm{H}$ & 0.4040428 & 4.7262485 & -1.4509370 \\
\hline $\mathrm{H}$ & -1.9905809 & 3.7150348 & -0.8400471 \\
\hline $\mathrm{C}$ & 1.1036595 & -0.1717982 & -2.7934745 \\
\hline C & -0.2069247 & 0.1998380 & -3.1616464 \\
\hline $\mathrm{C}$ & 1.3305483 & -1.4453433 & -2.2439034 \\
\hline C & 0.2780599 & -2.3657692 & -2.0442308 \\
\hline C & -1.2696038 & -0.7148664 & -2.9794140 \\
\hline $\mathrm{C}$ & -1.0264377 & -1.9921049 & -2.4363739 \\
\hline $\mathrm{Cl}$ & -0.5109609 & 1.7733548 & -3.8206440 \\
\hline $\mathrm{Cl}$ & -2.8810480 & -0.2614072 & -3.4109702 \\
\hline $\mathrm{Cl}$ & 2.4240674 & 0.9250980 & -3.0047091 \\
\hline $\mathrm{Cl}$ & -2.3104674 & -3.1200918 & -2.1823861 \\
\hline $\mathrm{Cl}$ & 2.9183445 & -1.9362501 & -1.7115620 \\
\hline 0 & 0.4791029 & -3.5744591 & -1.4882193 \\
\hline $\mathrm{H}$ & 1.4358901 & -3.6154263 & -1.2333443 \\
\hline
\end{tabular}

Vibrational frequencies

$\begin{array}{rrrrrr}0.00 & 0.00 & 0.00 & 0.00 & 0.00 & 0.00 \\ 2.14 & 8.83 & 15.57 & 19.45 & 27.08 & 44.96 \\ 50.30 & 57.97 & 74.64 & 83.77 & 85.84 & 91.54 \\ 103.39 & 115.12 & 137.71 & 144.78 & 154.09 & 166.63 \\ 170.11 & 177.25 & 199.11 & 203.73 & 208.54 & 212.25 \\ 213.86 & 220.09 & 234.44 & 266.05 & 271.07 & 272.73 \\ 279.70 & 285.01 & 300.79 & 307.32 & 314.85 & 322.27 \\ 330.97 & 333.01 & 337.07 & 344.46 & 358.87 & 359.82 \\ 370.23 & 372.06 & 402.69 & 405.01 & 419.40 & 420.97 \\ 422.47 & 424.39 & 457.64 & 458.07 & 499.19 & 539.63 \\ 540.46 & 564.27 & 635.27 & 636.58 & 650.04 & 652.78 \\ 653.70 & 667.39 & 673.71 & 691.62 & 692.85 & 694.31 \\ 697.04 & 713.51 & 723.92 & 734.01 & 734.72 & 756.94 \\ 762.06 & 768.91 & 769.06 & 774.40 & 778.37 & 785.44 \\ 786.30 & 799.92 & 807.91 & 841.18 & 849.80 & 850.87 \\ 859.30 & 866.20 & 886.06 & 891.16 & 891.73 & 893.73 \\ 975.49 & 994.86 & 1004.43 & 1005.02 & 1010.72 & 1017.27 \\ 1031.03 & 1033.10 & 1036.50 & 1040.50 & 1041.42 & 1043.11 \\ 1055.55 & 1111.82 & 1138.49 & 1148.49 & 1148.96 & 1183.04 \\ 1185.09 & 1238.01 & 1262.43 & 1262.77 & 1274.78 & 1294.37 \\ 1326.00 & 1326.78 & 1339.19 & 1357.19 & 1362.95 & 1371.12 \\ 1380.79 & 1385.95 & 1387.44 & 1396.29 & 1434.74 & 1463.95 \\ 1466.82 & 1468.08 & 1499.67 & 1521.11 & 1552.36 & 1553.34 \\ 1558.44 & 1572.53 & 1577.18 & 1581.24 & 1601.29 & 1602.41 \\ 1653.52 & 2549.34 & 3123.79 & 3124.67 & 3126.75 & 3128.77 \\ 3155.07 & 3156.02 & 3156.14 & 3158.08 & 3173.98 & 3174.77 \\ 3174.93 & 3176.78 & 3401.72 & & & \end{array}$

TURBOMOLE Energies and Thermochemistry ${ }^{1}$ RI-PBE/TZVP,SV(P) $-5326.81854$

ZPE

RI-PBE/TZVP,SV(P) + ZPE

0.3351935

RI-PBE/TZVPP

B3-LYP/TZVP,SV(P)

$\mathrm{ZPE}(\mathrm{kJ} / \mathrm{mol})$

$-5326.483346$

$-5329.252144$

$-5328.492435$

$\ln$ (qtrans)

880

$\ln$ (qrot)

20.39

In(qvib)

17.46

39.25

chem.pot. $(\mathrm{kJ} / \mathrm{mol})$

688.92

energy $(\mathrm{kJ} / \mathrm{mol})$

973.95

entropy $(\mathrm{kJ} / \mathrm{mol} / \mathrm{K})$

0.96433

Cv $(\mathrm{kJ} / \mathrm{mol}-\mathrm{K})$

0.5655578

$\mathrm{Cp}((\mathrm{kJ} / \mathrm{mol}-\mathrm{K})$

0.5738721

$\mathrm{RI}-\mathrm{PBE} / \mathrm{TZVP}, \mathrm{SV}(\mathrm{P})<\mathrm{S}^{2}>$

3.77

$\mathrm{RI}-\mathrm{PBE} / \mathrm{TZVPP}<\mathrm{S}^{2}>$

3.77

B3 - LYP / TZVP, SV (P) $<\mathrm{S}^{2}>$

3.79

${ }^{1}$ Freeh output; $298.15 \mathrm{~K}, 0.1 \mathrm{MPa}$, unscaled 
${ }^{4} \mathbf{A}$

\section{Pentachlorophenol: [(Porphyrin $)(\mathrm{Fe}=\mathbf{O})(\mathrm{SH})]$ Transition State (meta-oxidation)}

RI-PBE/TZVP, SV (P) Cartesian coordinates

$$
53
$$$$
\text { Energy }=-5326.794878634
$$

$\begin{array}{lrrr}\text { C } & -3.2556207 & -0.9979914 & 0.4307247 \\ \mathrm{~N} & -2.0227656 & -1.2264511 & 0.9948253 \\ \mathrm{C} & -1.9188248 & -2.5904098 & 1.1418230 \\ \mathrm{C} & -3.1147724 & -3.2444529 & 0.6491175 \\ \mathrm{C} & -3.9544365 & -2.2497569 & 0.2177247 \\ \mathrm{Fe} & -0.6176933 & 0.1586534 & 1.4402293 \\ \mathrm{O} & 0.1049806 & -0.0879541 & -0.1222910 \\ \mathrm{C} & -0.8492981 & -3.2557120 & 1.7455201 \\ \mathrm{C} & 0.2526033 & -2.6291041 & 2.3332180 \\ \mathrm{~N} & 0.4823581 & -1.2729635 & 2.3445180 \\ \mathrm{C} & 1.6398525 & -1.0880696 & 3.0684017 \\ \mathrm{C} & 2.1527740 & -2.3608853 & 3.5295031 \\ \mathrm{C} & 1.2940206 & -3.3230879 & 3.0615036 \\ \mathrm{C} & 2.2671808 & 0.1389727 & 3.2939304 \\ \mathrm{C} & 1.8355771 & 1.3684946 & 2.7929191 \\ \mathrm{~N} & 0.7131081 & 1.5655738 & 2.0189479 \\ \mathrm{C} & 0.7061195 & 2.9017096 & 1.6912225 \\ \mathrm{C} & 1.8554890 & 3.5684545 & 2.2672682 \\ \mathrm{C} & 2.5522096 & 2.6148267 & 2.9648996 \\ \mathrm{C} & -0.2918999 & 3.5433518 & 0.9540840 \\ \mathrm{C} & -1.4716389 & 2.9386118 & 0.5115238 \\ \mathrm{~N} & -1.7969922 & 1.6124626 & 0.6851232 \\ \mathrm{C} & -3.0607673 & 1.4597590 & 0.1608723 \\ \mathrm{C} & -3.5539747 & 2.7249876 & -0.3435258 \\ \mathrm{C} & -2.5566527 & 3.6442033 & -0.1386525 \\ \mathrm{C} & -3.7582167 & 0.2533505 & 0.0661094 \\ \mathrm{~S} & -1.5827431 & 0.4580131 & 3.5545341 \\ \mathrm{H} & -1.7305860 & 1.8207848 & 3.5023340 \\ \mathrm{H} & -2.5446972 & 4.7129615 & -0.3908041 \\ \mathrm{H} & -4.5380941 & 2.8709397 & -0.8083913 \\ \mathrm{H} & -4.7644190 & 0.2848262 & -0.3789714 \\ \mathrm{H} & -4.9588291 & -2.3382725 & -0.2172549 \\ \mathrm{H} & -3.2819607 & -4.3299366 & 0.6554758 \\ \mathrm{H} & -0.9037392 & -4.3533739 & 1.8098528 \\ \mathrm{H} & 1.3397975 & -4.4114493 & 3.2012722 \\ \mathrm{H} & 3.0626953 & -2.4862895 & 4.1314789 \\ \mathrm{H} & 3.1941254 & 0.1316266 & 3.8878310 \\ \mathrm{H} & 3.4785270 & 2.7272795 & 3.5438732 \\ \mathrm{H} & 2.0801904 & 4.6373752 & 2.1531417 \\ \mathrm{H} & -0.1661454 & 4.6187219 & 0.7549537 \\ \mathrm{C} & 1.6064634 & -0.2578986 & -1.2331694 \\ \mathrm{C} & 1.3121522 & 0.8346990 & -2.1422915 \\ \mathrm{C} & 1.3822893 & -1.5850074 & -1.7399254 \\ \mathrm{C} & 0.6301703 & -1.8230111 & -2.8973012 \\ \mathrm{C} & 0.5684922 & 0.6028358 & -3.3099226 \\ \mathrm{C} & 0.2271253 & -0.7165516 & -3.6948068 \\ \mathrm{C} 1 & 1.7771117 & 2.4330215 & -1.7060691 \\ \mathrm{Cl} & 0.1040832 & 1.9264208 & -4.3222516 \\ \mathrm{Cl} & 2.9641543 & -0.0541890 & -0.1433974 \\ \mathrm{Cl} & -0.6788622 & -1.0282051 & -5.1265647 \\ \mathrm{Cl} & 1.8739051 & -2.9759667 & -0.8147720 \\ \mathrm{O} & 0.3075150 & -3.0653033 & -3.3073879 \\ \mathrm{H} & 0.6563629 & -3.6896731 & -2.6206436\end{array}$

Vibrational frequencies

$\begin{array}{rrrrrr}i 506.91 & 0.00 & 0.00 & 0.00 & 0.00 & 0.00 \\ 0.00 & 7.78 & 22.38 & 34.88 & 39.53 & 49.50 \\ 52.81 & 54.15 & 72.81 & 79.48 & 87.33 & 100.12 \\ 102.83 & 109.35 & 126.58 & 142.82 & 148.68 & 157.72 \\ 194.69 & 199.06 & 201.46 & 207.90 & 211.08 & 213.54 \\ 223.79 & 233.96 & 240.53 & 252.32 & 270.82 & 272.51 \\ 281.30 & 292.66 & 293.53 & 299.45 & 316.78 & 320.76 \\ 326.94 & 344.58 & 347.96 & 354.93 & 359.96 & 362.75 \\ 364.18 & 372.05 & 409.67 & 413.54 & 418.54 & 418.75 \\ 420.35 & 421.48 & 439.36 & 450.33 & 458.59 & 515.32 \\ 573.59 & 615.59 & 627.49 & 639.87 & 652.70 & 655.98 \\ 657.12 & 672.07 & 673.65 & 685.01 & 694.76 & 695.04 \\ 697.30 & 701.72 & 716.93 & 724.62 & 734.75 & 736.09 \\ 760.31 & 764.52 & 771.54 & 771.65 & 777.44 & 780.82 \\ 784.81 & 785.29 & 799.18 & 841.65 & 849.84 & 851.79 \\ 852.32 & 859.71 & 886.40 & 891.53 & 892.28 & 894.29 \\ 962.05 & 994.08 & 1003.00 & 1003.61 & 1010.36 & 1015.75 \\ 1031.37 & 1032.64 & 1036.11 & 1041.13 & 1041.43 & 1042.65 \\ 1054.52 & 1111.23 & 1140.25 & 1147.84 & 1150.22 & 1183.76 \\ 1183.97 & 1189.24 & 1233.21 & 1262.01 & 1264.38 & 1307.34 \\ 1314.16 & 1328.69 & 1329.32 & 1340.05 & 1364.54 & 1373.10 \\ 1377.73 & 1382.78 & 1385.80 & 1391.98 & 1393.09 & 1468.27 \\ 1469.30 & 1470.41 & 1501.78 & 1508.96 & 1524.03 & 1546.70 \\ 1555.75 & 1556.37 & 1576.84 & 1591.66 & 1606.18 & 1609.26 \\ 1660.06 & 2549.56 & 3121.98 & 3122.19 & 3123.73 & 3124.79 \\ 3153.97 & 3154.39 & 3154.71 & 3155.29 & 3173.08 & 3173.64 \\ 3173.81 & 3174.30 & 3394.08 & & & \end{array}$

TURBOMOLE Energies and Thermochemistry ${ }^{1}$ RI-PBE/TZVP,SV(P) $-5326.794879$

ZPE

RI-PBE/TZVP,SV(P) + ZPE

0.3346681

RI-PBE/TZVPP

B3-LYP/TZVP,SV(P)

$-5326.460211$

$-5329.225798$

ZPE $(\mathrm{kJ} / \mathrm{mol})$

$-5328.460923$

In(qtrans)

878.7

$\ln$ (qrot)

20.39

In(qvib)

17.43

33.41

chem.pot. $(\mathrm{kJ} / \mathrm{mol})$

702.09

energy $(\mathrm{kJ} / \mathrm{mol})$

969.81

entropy $(\mathrm{kJ} / \mathrm{mol} / \mathrm{K})$

0.90624

Cv $(\mathrm{kJ} / \mathrm{mol}-\mathrm{K})$

0.5578702

$\mathrm{Cp}((\mathrm{kJ} / \mathrm{mol}-\mathrm{K})$

0.5661845

$\mathrm{RI}-\mathrm{PBE} / \mathrm{TZVP}, \mathrm{SV}(\mathrm{P})<\mathrm{S}^{2}>$

3.77

$\mathrm{RI}-\mathrm{PBE} / \mathrm{TZVPP}<\mathrm{S}^{2}>$

3.77

B3 - LYP /TZVP, SV (P) $<\mathrm{S}^{2}>$

3.80

${ }^{1}$ Freeh output; $298.15 \mathrm{~K}, 0.1 \mathrm{MPa}$, unscaled 
${ }^{4} \mathrm{~A}$

\section{Pentachlorophenol: [(Porphyrin $)(\mathrm{Fe}=\mathbf{O})(\mathrm{SH})]$ Product Complex (meta-oxidation)}

\begin{tabular}{|c|c|c|c|}
\hline \multicolumn{2}{|c|}{ RI - PBE/TZVP, SV (P) } & Cartesian & coordinates \\
\hline 53 & & & \\
\hline & $g y=-5326.8$ & 2415086 & \\
\hline $\mathrm{C}$ & -2.8113404 & 0.4641481 & 2.5603655 \\
\hline & -1.7159521 & -0.3615810 & 2.4461993 \\
\hline C & -2.1310929 & -1.6068822 & 2.8669182 \\
\hline $\mathrm{C}$ & -3.5307523 & -1.5714370 & 3.2323058 \\
\hline C & -3.9586494 & -0.2835961 & 3.0285197 \\
\hline $\mathrm{Fe}$ & 0.1430058 & 0.1505984 & 1.8169457 \\
\hline $\mathrm{O}$ & -0.5361007 & -0.1696497 & -0.0026375 \\
\hline $\mathrm{C}$ & -1.3306865 & -2.7470286 & 2.9330255 \\
\hline $\mathrm{C}$ & -0.0026675 & -2.8129139 & 2.5083773 \\
\hline $\mathrm{N}$ & 0.7309292 & -1.7652797 & 1.9852411 \\
\hline $\mathrm{C}$ & 1.9381436 & -2.3030936 & 1.5874908 \\
\hline $\mathrm{C}$ & 1.9723239 & -3.7168539 & 1.8741706 \\
\hline $\mathrm{C}$ & 0.7695227 & -4.0313344 & 2.4616597 \\
\hline $\mathrm{C}$ & 2.9856293 & -1.5940110 & 0.9979671 \\
\hline C & 2.9891861 & -0.2156623 & 0.8013177 \\
\hline $\mathrm{N}$ & 1.9547164 & 0.6410437 & 1.1087085 \\
\hline C & 2.4458079 & 1.9127843 & 0.8996221 \\
\hline $\mathrm{C}$ & 3.8074883 & 1.8569666 & 0.4212032 \\
\hline $\mathrm{C}$ & 4.1406072 & 0.5300410 & 0.3402027 \\
\hline $\mathrm{C}$ & 1.7420468 & 3.0952592 & 1.1192679 \\
\hline $\mathrm{C}$ & 0.4075899 & 3.1567817 & 1.5190021 \\
\hline $\mathrm{N}$ & -0.4015302 & 2.0680298 & 1.7969520 \\
\hline $\mathrm{C}$ & -1.6662698 & 2.5824923 & 2.0206150 \\
\hline $\mathrm{C}$ & -1.6483226 & 4.0202483 & 1.8938378 \\
\hline $\mathrm{C}$ & -0.3546766 & 4.3786864 & 1.5985831 \\
\hline $\mathrm{C}$ & -2.8044574 & 1.8419095 & 2.3400067 \\
\hline$S$ & 0.8443887 & 0.3221175 & 3.92 \\
\hline $\mathrm{H}$ & 0.2316866 & 1.5126989 & 4.2268480 \\
\hline $\mathrm{H}$ & 0.0560049 & 5.3812035 & 1.4196436 \\
\hline $\mathrm{H}$ & -2.5280193 & 4.6658298 & 2.0172219 \\
\hline $\mathrm{H}$ & -3.7490521 & 2.3896918 & 2.4756340 \\
\hline $\mathrm{H}$ & -4.9566393 & 0.1438497 & 3.1931987 \\
\hline $\mathrm{H}$ & -4.1019169 & -2.4359822 & 3.5960358 \\
\hline $\mathrm{H}$ & -1.7907798 & -3.6731537 & 3.3093258 \\
\hline $\mathrm{H}$ & 0.4145694 & -5.0076331 & 2.8180090 \\
\hline $\mathrm{H}$ & 2.8204526 & -4.3771184 & 1.6496674 \\
\hline $\mathrm{H}$ & 3.8884131 & -2.1545851 & 0.7134397 \\
\hline $\mathrm{H}$ & 5.0833209 & 0.0752119 & 0.0097778 \\
\hline $\mathrm{H}$ & 4.412 & 2.7354247 & 2408 \\
\hline $\mathrm{H}$ & 2.2593748 & 4.0449233 & 77345 \\
\hline $\mathrm{C}$ & -0.4689096 & -0.3438027 & -1.2683567 \\
\hline C & -0.4383045 & 0.7769446 & -2.2123821 \\
\hline $\mathrm{C}$ & -0.5122989 & -1.6419910 & -1.8665191 \\
\hline C & -0.6172556 & -1.8549108 & -3.2513727 \\
\hline $\mathrm{C}$ & -0.6024463 & 0.5665026 & -3.6131670 \\
\hline $\mathrm{C}$ & -0.6581940 & -0.7347358 & -4.1318255 \\
\hline $\mathrm{Cl}$ & -0.6255515 & 2.3595213 & -1.5651385 \\
\hline $\mathrm{Cl}$ & -0.6858829 & 1.9191794 & -4.6766250 \\
\hline $\mathrm{Cl}$ & 2.1797601 & 0.5246097 & -2.3618089 \\
\hline $\mathrm{Cl}$ & -0.7855961 & -1.0317568 & -5.8259831 \\
\hline $\mathrm{Cl}$ & -0.5150254 & -3.0616183 & -0.8519090 \\
\hline $\mathrm{O}$ & -0.6798058 & -3.0876468 & -3.7803535 \\
\hline $\mathrm{H}$ & -0.6262651 & -3.7239146 & -3.0191939 \\
\hline
\end{tabular}

Vibrational frequencies

$\begin{array}{rrrrrr}0.00 & 0.00 & 0.00 & 0.00 & 0.00 & 0.00 \\ 4.80 & 26.06 & 39.84 & 43.62 & 51.05 & 56.54 \\ 56.88 & 62.51 & 75.78 & 79.09 & 90.10 & 96.85 \\ 104.97 & 109.14 & 114.64 & 128.06 & 142.17 & 147.54 \\ 154.68 & 173.30 & 197.48 & 199.48 & 210.84 & 211.40 \\ 216.88 & 231.60 & 248.17 & 254.44 & 268.23 & 270.53 \\ 272.21 & 277.12 & 285.10 & 291.67 & 298.98 & 305.58 \\ 331.76 & 341.57 & 348.58 & 358.48 & 359.47 & 360.58 \\ 370.59 & 376.15 & 394.04 & 399.45 & 401.15 & 415.77 \\ 417.75 & 422.84 & 423.47 & 454.35 & 533.95 & 556.22 \\ 561.39 & 628.97 & 635.28 & 645.50 & 652.17 & 653.77 \\ 667.43 & 670.49 & 673.50 & 682.07 & 692.86 & 694.29 \\ 694.95 & 717.32 & 722.97 & 730.71 & 734.74 & 743.81 \\ 760.42 & 769.24 & 772.02 & 775.49 & 779.39 & 780.28 \\ 783.27 & 786.26 & 797.96 & 834.15 & 843.23 & 844.42 \\ 851.89 & 888.49 & 892.81 & 894.22 & 895.45 & 918.21 \\ 993.06 & 997.48 & 1000.98 & 1013.68 & 1018.32 & 1030.16 \\ 1033.09 & 1036.84 & 1042.26 & 1042.96 & 1043.48 & 1054.13 \\ 1105.83 & 1127.07 & 1138.16 & 1142.40 & 1144.71 & 1181.25 \\ 1184.21 & 1241.38 & 1259.98 & 1262.44 & 1293.70 & 1320.92 \\ 1330.29 & 1330.41 & 1333.53 & 1348.74 & 1365.19 & 1366.71 \\ 1374.26 & 1381.81 & 1384.93 & 1412.76 & 1454.74 & 1465.15 \\ 1469.86 & 1476.04 & 1510.54 & 1511.20 & 1517.85 & 1539.19 \\ 1548.41 & 1556.78 & 1578.25 & 1595.58 & 1614.81 & 1623.77 \\ 1663.49 & 2542.54 & 3126.16 & 3128.45 & 3131.41 & 3131.47 \\ 3156.05 & 3156.36 & 3157.07 & 3162.85 & 3174.91 & 3175.08 \\ 3175.55 & 3181.55 & 3360.94 & & & \end{array}$

TURBOMOLE Energies and Thermochemistry ${ }^{1}$

RI-PBE/TZVP,SV(P)

$-5326.842415$

ZPE

0.3357018

RI-PBE/TZVP,SV(P) + ZPE

$-5326.506713$

RI-PBE/TZVPP

B3-LYP/TZVP,SV(P)

$-5329.270316$

$\mathrm{ZPE}(\mathrm{kJ} / \mathrm{mol})$

$-5328.520162$

$\ln$ (qtrans)

881.4

In(qrot)

20.39

In(qvib)

17.51

35.29

chem.pot. $(\mathrm{kJ} / \mathrm{mol})$

699.95

energy $(\mathrm{kJ} / \mathrm{mol})$

975.08

entropy $(\mathrm{kJ} / \mathrm{mol} / \mathrm{K})$

0.93112

Cv $(\mathrm{kJ} / \mathrm{mol}-\mathrm{K})$

0.5649243

$\mathrm{Cp}((\mathrm{kJ} / \mathrm{mol}-\mathrm{K})$

0.5732386

$\mathrm{RI}-\mathrm{PBE} / \mathrm{TZVP}, \mathrm{SV}(\mathrm{P})<\mathrm{S}^{2}>$

3.78

$\mathrm{RI}-\mathrm{PBE} / \mathrm{TZVPP}<\mathrm{S}^{2}>$

3.79

B3 - LYP/TZVP, SV (P) $\quad\left\langle\mathrm{S}^{2}>\right.$

3.92

${ }^{1}$ Freeh output; $298.15 \mathrm{~K}, 0.1 \mathrm{MPa}$, unscaled 
Pentachlorophenol: [(Porphyrin $)(\mathrm{Fe}=\mathbf{O})(\mathrm{SH})]$ Prereactant Complex (ortho-oxidation)

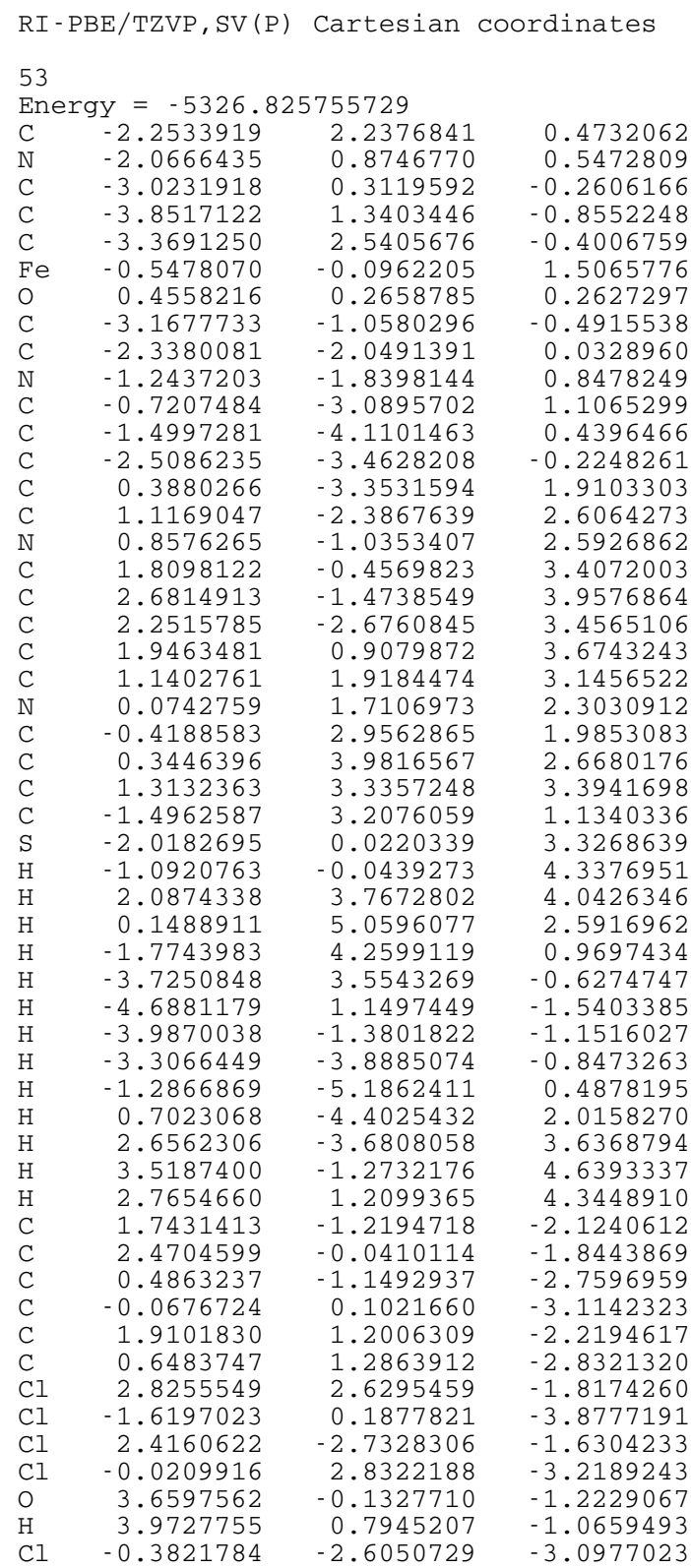

$\begin{array}{rrrrrr}i 10.15 & 0.00 & 0.00 & 0.00 & 0.00 & 0.00 \\ 0.00 & 5.74 & 11.96 & 19.01 & 26.74 & 36.64 \\ 47.10 & 55.91 & 73.75 & 83.87 & 95.01 & 99.30 \\ 101.95 & 115.22 & 121.21 & 146.46 & 149.66 & 172.40 \\ 179.10 & 199.13 & 203.36 & 205.96 & 208.53 & 213.95 \\ 216.71 & 234.48 & 238.88 & 271.88 & 275.14 & 276.83 \\ 279.50 & 284.90 & 299.88 & 301.19 & 309.89 & 322.11 \\ 331.41 & 333.28 & 336.94 & 345.72 & 358.83 & 359.40 \\ 364.61 & 372.34 & 396.43 & 401.50 & 418.20 & 419.13 \\ 419.64 & 421.91 & 458.23 & 459.13 & 498.90 & 542.47 \\ 555.83 & 605.25 & 629.78 & 636.05 & 649.75 & 656.24 \\ 656.86 & 672.82 & 679.49 & 693.79 & 694.63 & 694.93 \\ 697.43 & 715.52 & 723.77 & 733.99 & 735.21 & 757.80 \\ 763.68 & 771.54 & 771.96 & 777.97 & 781.85 & 784.38 \\ 784.61 & 798.41 & 840.57 & 849.97 & 850.14 & 851.23 \\ 858.52 & 867.35 & 886.56 & 891.74 & 892.25 & 894.38 \\ 977.33 & 993.00 & 1001.92 & 1002.59 & 1011.09 & 1015.23 \\ 1031.24 & 1032.67 & 1035.50 & 1040.75 & 1041.26 & 1042.30 \\ 1054.49 & 1113.12 & 1140.64 & 1148.83 & 1149.38 & 1183.11 \\ 1184.09 & 1237.07 & 1261.55 & 1263.19 & 1274.08 & 1314.90 \\ 1329.30 & 1330.78 & 1341.06 & 1355.31 & 1363.73 & 1376.42 \\ 1383.22 & 1386.34 & 1391.00 & 1393.52 & 1434.32 & 1469.19 \\ 1472.10 & 1472.99 & 1504.63 & 1525.16 & 1556.80 & 1557.75 \\ 1558.29 & 1575.48 & 1579.30 & 1603.37 & 1609.25 & 1610.89 \\ 1662.35 & 2545.90 & 3123.17 & 3123.24 & 3127.01 & 3130.30 \\ 3154.34 & 3155.67 & 3156.83 & 3157.05 & 3173.32 & 3174.71 \\ 3175.85 & 3176.20 & 3398.98 & & & \end{array}$

TURBOMOLE Energies and Thermochemistry ${ }^{1}$

\begin{tabular}{|c|c|}
\hline RI-PBE/TZVP,SV(P) & -5326.825756 \\
\hline ZPE & 0.335784 \\
\hline RI-PBE/TZVP,SV(P) + ZPE & -5326.489972 \\
\hline RI-PBE/TZVPP & -5329.258824 \\
\hline B3-LYP/TZVP,SV(P) & -5328.493388 \\
\hline ZPE (kJ/mol) & 881.6 \\
\hline In(qtrans) & 20.39 \\
\hline $\ln ($ qrot $)$ & 17.44 \\
\hline $\ln (q v i b)$ & 35.42 \\
\hline chem.pot. $(\mathrm{kJ} / \mathrm{mol})$ & 700.02 \\
\hline energy (kJ/mol) & 972.78 \\
\hline entropy $(\mathrm{kJ} / \mathrm{mol} / \mathrm{K})$ & 0.92316 \\
\hline $\mathrm{Cv}(\mathrm{kJ} / \mathrm{mol}-\mathrm{K})$ & 0.5559261 \\
\hline Cp $((\mathrm{kJ} / \mathrm{mol}-\mathrm{K})$ & 0.5642404 \\
\hline $\mathrm{RI}-\mathrm{PBE} / \mathrm{TZVP}, \mathrm{SV}(\mathrm{P}) \quad<\mathrm{S}^{2}>$ & 1.10 \\
\hline $\mathrm{RI}-\mathrm{PBE} / \mathrm{TZVPP} \quad<\mathrm{S}^{2}>$ & 1.12 \\
\hline B3 - LYP / TZVP, SV (P) $\quad<\mathrm{S}^{2}>$ & 1.64 \\
\hline
\end{tabular}

${ }^{1}$ Freeh output; $298.15 \mathrm{~K}, 0.1 \mathrm{MPa}$, unscaled 
Pentachlorophenol: [(Porphyrin $)(\mathrm{Fe}=\mathbf{O})(\mathrm{SH})]$ Transition State (ortho-oxidation)

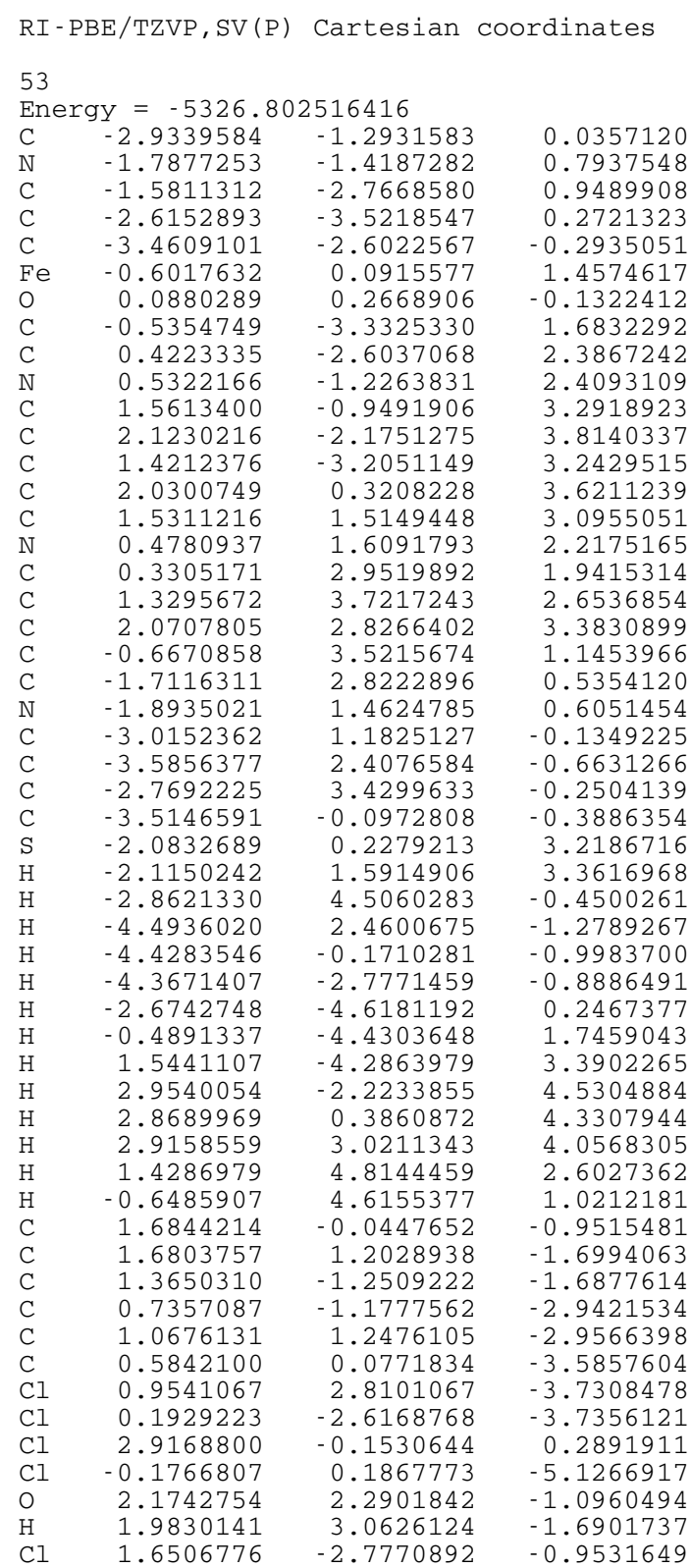

$\begin{array}{rrrrrr}1520.18 & 0.00 & 0.00 & 0.00 & 0.00 & 0.00 \\ 0.00 & 7.22 & 17.23 & 27.78 & 44.92 & 48.39 \\ 51.07 & 58.79 & 70.53 & 82.38 & 87.40 & 88.07 \\ 105.45 & 118.47 & 127.85 & 146.11 & 147.25 & 157.72 \\ 168.89 & 195.58 & 199.37 & 205.76 & 207.42 & 211.86 \\ 215.43 & 231.63 & 251.86 & 256.34 & 263.72 & 272.63 \\ 277.01 & 286.57 & 295.50 & 299.40 & 312.46 & 321.94 \\ 328.21 & 338.42 & 349.54 & 350.54 & 358.14 & 359.78 \\ 366.10 & 388.17 & 394.91 & 412.74 & 418.51 & 420.31 \\ 422.12 & 424.15 & 452.95 & 458.85 & 493.54 & 530.56 \\ 590.71 & 595.75 & 636.36 & 647.07 & 651.66 & 656.56 \\ 657.78 & 672.93 & 678.21 & 680.36 & 692.11 & 695.14 \\ 698.67 & 699.33 & 717.48 & 723.99 & 734.38 & 735.65 \\ 750.93 & 763.56 & 770.33 & 771.51 & 777.24 & 782.96 \\ 784.74 & 785.22 & 798.81 & 840.08 & 849.02 & 849.18 \\ 856.76 & 858.84 & 884.09 & 889.16 & 890.95 & 893.58 \\ 980.16 & 991.42 & 1000.74 & 1001.59 & 1010.31 & 1013.50 \\ 1030.93 & 1031.76 & 1035.39 & 1040.39 & 1041.06 & 1041.89 \\ 1053.84 & 1075.69 & 1139.26 & 1147.90 & 1149.08 & 1182.54 \\ 1183.19 & 1211.49 & 1248.87 & 1260.99 & 1262.73 & 1321.39 \\ 1322.12 & 1329.17 & 1331.66 & 1341.99 & 1361.81 & 1363.34 \\ 1373.71 & 1383.56 & 1391.92 & 1393.48 & 1416.38 & 1469.52 \\ 1471.62 & 1472.87 & 1490.15 & 1504.99 & 1524.20 & 1543.41 \\ 1557.34 & 1558.74 & 1579.65 & 1606.89 & 1610.38 & 1614.00 \\ 1661.06 & 2549.73 & 3118.12 & 3121.13 & 3122.90 & 3125.11 \\ 3152.23 & 3153.02 & 3153.87 & 3154.73 & 3171.50 & 3172.48 \\ 3173.15 & 3173.89 & 3373.04 & & & \end{array}$

TURBOMOLE Energies and Thermochemistry ${ }^{1}$

\begin{tabular}{|c|c|}
\hline RI-PBE/TZVP,SV(P) & -5326.802516 \\
\hline ZPE & 0.3347396 \\
\hline RI-PBE/TZVP,SV(P) + ZPE & -5326.467777 \\
\hline RI-PBE/TZVPP & -5329.233067 \\
\hline B3-LYP/TZVP,SV(P) & -5328.462314 \\
\hline $\mathrm{ZPE}(\mathrm{kJ} / \mathrm{mol})$ & 878.8 \\
\hline In(qtrans) & 20.39 \\
\hline In(qrot) & 17.43 \\
\hline $\ln (q v i b)$ & 33.93 \\
\hline chem.pot. (kJ/mol) & 701.02 \\
\hline energy (kJ/mol) & 970.09 \\
\hline entropy $(\mathrm{kJ} / \mathrm{mol} / \mathrm{K})$ & 0.91078 \\
\hline $\mathrm{Cv}(\mathrm{kJ} / \mathrm{mol}-\mathrm{K})$ & 0.5575058 \\
\hline $\mathrm{Cp}((\mathrm{kJ} / \mathrm{mol}-\mathrm{K})$ & 0.5658201 \\
\hline $\mathrm{RI}-\mathrm{PBE} / \mathrm{TZVP}, \mathrm{SV}(\mathrm{P}) \quad\left\langle\mathrm{S}^{2}>\right.$ & 0.92 \\
\hline $\mathrm{RI}-\mathrm{PBE} / \mathrm{TZVPP}<\mathrm{S}^{2}>$ & 0.91 \\
\hline B3 - LYP/TZVP, SV (P) $\left\langle\mathrm{S}^{2}>\right.$ & 1.46 \\
\hline
\end{tabular}

${ }^{1}$ Freeh output; $298.15 \mathrm{~K}, 0.1 \mathrm{MPa}$, unscaled 
${ }^{2} \mathbf{A}$

\section{Pentachlorophenol:[(Porphyrin $)(\mathrm{Fe}=\mathbf{O})(\mathrm{SH})]$ Product Complex (ortho-oxidation)}

\begin{tabular}{|c|c|c|c|}
\hline \multicolumn{2}{|c|}{ RI - PBE/TZVP, SV (P) } & Cartesian & coordinates \\
\hline & & & \\
\hline & $I=-5326.86$ & 584771 & \\
\hline $\mathrm{C}$ & -2.9560638 & 0.9162475 & 2.1187670 \\
\hline & -2.0055359 & -0.0846518 & 2.1634151 \\
\hline & -2.7049174 & -1.2532919 & 2.3956380 \\
\hline & -4.1229777 & -0.9816401 & 2.5075524 \\
\hline & -4.2793351 & 0.3696089 & 2.3352935 \\
\hline $\mathrm{Fe}$ & -0.0175447 & 0.1195567 & 1.9642892 \\
\hline & -0.1420066 & -0.2228465 & -0.0170128 \\
\hline & -2.1468083 & -2.5280564 & 2.4835690 \\
\hline C & -0.7845559 & -2.8111723 & 2.3698906 \\
\hline & 0.2099600 & -1.8817320 & 2.1635100 \\
\hline C & 1.3927400 & -2.5882587 & 2.1644211 \\
\hline $\mathrm{C}$ & 1.1385026 & -4.0004544 & 2.3530386 \\
\hline $\mathrm{C}$ & -0.2208570 & -4.1396206 & 2.4805315 \\
\hline $\mathrm{C}$ & 2.6656986 & -2.0348729 & 2.0211250 \\
\hline $\mathrm{C}$ & 2.9281204 & -0.6752280 & 1.8518659 \\
\hline N & 1.9765162 & 0.3233960 & 1.7825691 \\
\hline C & 2.6800151 & 1.4939986 & 1.5803051 \\
\hline C & 4.1027625 & 1.2307058 & 1.5326089 \\
\hline $\mathrm{C}$ & 4.2575589 & -0.1210311 & 1.7015786 \\
\hline C & 2.1148066 & 2.7579885 & 1.4126353 \\
\hline $\mathrm{C}$ & 0.7462644 & 3.0266059 & 1.4389346 \\
\hline $\mathrm{N}$ & -0.2487088 & 2.0970470 & 1.6488048 \\
\hline $\mathrm{C}$ & -1.4306899 & 2.8033736 & 1.6487916 \\
\hline C & -1.1811811 & 4.2077741 & 1.4059084 \\
\hline $\mathrm{C}$ & 0.1780026 & 4.3469762 & 1.2740464 \\
\hline C & -2.6985488 & 2.2638780 & 1.87 \\
\hline S & 0.0758356 & 0.4058598 & 4.12 \\
\hline $\mathrm{H}$ & 1.4344886 & 1838 & 4.27 \\
\hline $\mathrm{H}$ & 0.7629873 & 5.2590913 & 1.09 \\
\hline $\mathrm{H}$ & -1.96 & 4.9809985 & 1. 3592551 \\
\hline $\mathrm{H}$ & -3.5569977 & 2.9526607 & 1.8545327 \\
\hline $\mathrm{H}$ & -5.2041308 & 0.9619639 & 2.3457596 \\
\hline $\mathrm{H}$ & -4.8911692 & -1.7451279 & 2.6904001 \\
\hline $\mathrm{H}$ & -2.8309172 & -3.3720012 & 2.6598994 \\
\hline $\mathrm{H}$ & -0.8067115 & -5.0541789 & 2.6436004 \\
\hline $\mathrm{H}$ & 1.9158965 & -4.7757602 & 2.3872779 \\
\hline $\mathrm{H}$ & 3.5263312 & -2.7204789 & 2.0491935 \\
\hline $\mathrm{H}$ & 5.1835373 & -0.7114006 & 1.7156210 \\
\hline $\mathrm{H}$ & 4.873 & 1.9973698 & 1.37 \\
\hline $\mathrm{H}$ & 2.7986741 & 3.6034826 & 1.2434378 \\
\hline C & -0.0730816 & -0.22003 & -1.2552480 \\
\hline C & -0.0224622 & 1.1038063 & -2.0273589 \\
\hline C & 0.0001910 & -1.4451692 & -2.0419446 \\
\hline C & -0.1461472 & -1.4325531 & -3.4228630 \\
\hline $\mathrm{C}$ & -0.3343273 & 1.0101293 & -3.4884226 \\
\hline $\mathrm{C}$ & -0.3449480 & -0.1860210 & -4.1553578 \\
\hline $\mathrm{Cl}$ & -0.5812542 & 2.5251144 & -4.2902260 \\
\hline $\mathrm{Cl}$ & -0.1053292 & -2.9052124 & -4.3139591 \\
\hline $\mathrm{Cl}$ & 1.9008948 & 1.4629753 & -2.0126380 \\
\hline $\mathrm{Cl}$ & -0.6165599 & -0.2461658 & -5.8600165 \\
\hline 0 & -0.6850630 & 2.0743793 & -1.3538519 \\
\hline $\mathrm{H}$ & -0.7298669 & 2.8708617 & -1.9405973 \\
\hline $\mathrm{Cl}$ & 0.1675211 & -2.9210402 & -1.1714039 \\
\hline
\end{tabular}

Vibrational frequencies

$\begin{array}{rrrrrr}0.00 & 0.00 & 0.00 & 0.00 & 0.00 & 0.00 \\ 4.00 & 17.11 & 33.92 & 36.78 & 46.33 & 53.58 \\ 57.10 & 62.05 & 69.56 & 75.70 & 96.10 & 105.17 \\ 112.03 & 116.98 & 131.70 & 146.77 & 149.59 & 187.49 \\ 200.67 & 207.99 & 216.84 & 217.66 & 228.41 & 229.42 \\ 243.99 & 258.91 & 263.89 & 272.86 & 273.47 & 277.22 \\ 278.75 & 290.07 & 296.12 & 302.74 & 313.97 & 321.85 \\ 337.48 & 342.10 & 352.21 & 358.41 & 359.52 & 360.10 \\ 387.67 & 398.37 & 399.70 & 412.37 & 419.52 & 420.07 \\ 420.51 & 421.32 & 456.37 & 459.74 & 486.27 & 507.62 \\ 568.77 & 611.61 & 652.78 & 657.50 & 658.44 & 664.87 \\ 674.16 & 685.33 & 695.16 & 697.37 & 697.63 & 700.86 \\ 719.75 & 721.93 & 724.25 & 734.82 & 735.72 & 739.04 \\ 761.78 & 770.00 & 770.22 & 777.18 & 784.51 & 785.33 \\ 786.33 & 799.06 & 808.36 & 834.28 & 842.81 & 843.05 \\ 850.48 & 883.57 & 887.79 & 890.07 & 891.19 & 901.07 \\ 974.58 & 991.60 & 999.50 & 1001.96 & 1009.27 & 1011.93 \\ 1029.68 & 1031.46 & 1034.42 & 1039.71 & 1040.59 & 1041.59 \\ 1054.16 & 1111.48 & 1139.57 & 1145.38 & 1147.75 & 1176.22 \\ 1182.58 & 1183.06 & 1260.61 & 1262.26 & 1265.34 & 1271.65 \\ 1328.77 & 1330.72 & 1335.10 & 1344.90 & 1355.50 & 1365.82 \\ 1375.73 & 1387.71 & 1389.27 & 1390.53 & 1470.99 & 1471.61 \\ 1472.23 & 1475.91 & 1508.14 & 1522.61 & 1557.66 & 1560.35 \\ 1568.68 & 1580.71 & 1610.16 & 1613.31 & 1614.93 & 1622.95 \\ 1661.83 & 2513.39 & 3121.47 & 3122.48 & 3123.43 & 3124.40 \\ 3149.71 & 3151.61 & 3152.04 & 3152.44 & 3169.45 & 3171.02 \\ 3171.43 & 3171.83 & 3426.23 & & & \end{array}$

TURBOMOLE Energies and Thermochemistry ${ }^{1}$

\begin{tabular}{|c|c|}
\hline RI-PBE/TZVP,SV(P) & -5326.862585 \\
\hline ZPE & 0.3365917 \\
\hline RI-PBE/TZVP,SV(P) + ZPE & -5326.525993 \\
\hline RI-PBE/TZVPP & -5329.289269 \\
\hline B3-LYP/TZVP,SV(P) & -5328.540254 \\
\hline ZPE (kJ/mol) & 883.7 \\
\hline $\ln ($ qtrans) & 20.39 \\
\hline In(qrot) & 17.57 \\
\hline In(qvib) & 34.94 \\
\hline chem.pot. (kJ/mol) & 703.02 \\
\hline energy (kJ/mol) & 975.62 \\
\hline entropy $(\mathrm{kJ} / \mathrm{mol} / \mathrm{K})$ & 0.9226 \\
\hline $\mathrm{Cv}(\mathrm{kJ} / \mathrm{mol}-\mathrm{K})$ & 0.5617544 \\
\hline $\mathrm{Cp}((\mathrm{kJ} / \mathrm{mol}-\mathrm{K})$ & 0.5700687 \\
\hline $\mathrm{RI}-\mathrm{PBE} / \mathrm{TZVP}, \mathrm{SV}(\mathrm{P})<\mathrm{S}^{2}>$ & 0.90 \\
\hline $\mathrm{RI}-\mathrm{PBE} / \mathrm{TZVPP}<\mathrm{S}^{2}>$ & 0.90 \\
\hline B3 - LYP/TZVP, SV (P) $\left\langle\mathrm{S}^{2}>\right.$ & 0.80 \\
\hline
\end{tabular}

${ }^{1}$ Freeh output; 298.15K, 0.1MPa, unscaled 
2-Hydroxy-2,3,4,5,6-pentachloro-3,5cyclohexadieneone

\begin{tabular}{lrrr} 
lu & \multicolumn{3}{l}{} \\
Energy $=-2678.284088027$ & \\
C & -1.4665556 & -0.5452601 & -0.1545470 \\
C & -0.4958332 & -1.5880028 & -0.4859624 \\
C & 0.9966819 & -1.2564986 & -0.2506944 \\
C & 1.3553420 & 0.1937437 & -0.2501882 \\
C & 0.3770637 & 1.1447831 & -0.1100163 \\
C & -1.0414703 & 0.7648962 & -0.0251067 \\
O & -0.7552560 & -2.6998512 & -0.9348292 \\
Cl & 3.0214693 & 0.5789077 & -0.3494083 \\
Cl & 0.7890959 & 2.8185827 & -0.0259985 \\
Cl & -2.1946661 & 2.0114189 & 0.2393921 \\
Cl & -3.1250821 & -0.9976374 & -0.1299387 \\
Cl & 1.1050973 & -1.7584305 & 1.6154261 \\
O & 1.7806908 & -2.0341636 & -1.0205809 \\
H & 1.2103849 & -2.8367801 & -1.2153685
\end{tabular}

Vibrational frequencies

$\begin{array}{rrrrrr}0.00 & 0.00 & 0.00 & 0.00 & 0.00 & 0.00 \\ 53.80 & 63.39 & 84.15 & 119.55 & 159.85 & 192.23 \\ 196.84 & 219.39 & 241.91 & 265.81 & 296.45 & 312.98 \\ 330.75 & 355.13 & 359.62 & 406.95 & 484.28 & 505.81 \\ 586.69 & 624.84 & 660.94 & 680.75 & 753.74 & 769.63 \\ 810.93 & 923.62 & 979.51 & 1127.49 & 1180.11 & 1252.47 \\ 1312.14 & 1405.35 & 1499.77 & 1569.96 & 1719.30 & 3227.32\end{array}$

TURBOMOLE Energies and Thermochemistry ${ }^{1}$

\begin{tabular}{lr}
\hline RI-PBE/TZVP,SV(P) & -1261.451976 \\
\hline ZPE & 0.0527456 \\
RI-PBE/TZVP,SV(P) + ZPE & -1261.39923 \\
RI-PBE/TZVPP & -1262.555005 \\
B3-LYP/TZVP,SV(P) & -1262.028322 \\
ZPE (kJ/mol) & 138.5 \\
In(qtrans) & 18.68 \\
In(qrot) & 14.35 \\
In(qvib) & 7.41 \\
chem.pot. (kJ/mol) & 38.21 \\
energy (kJ/mol) & 168.49 \\
entropy $(\mathrm{kJ} / \mathrm{mol} / \mathrm{K})$ & 0.44529 \\
CV $(\mathrm{kJ} / \mathrm{mol}-\mathrm{K})$ & 0.1681613 \\
Cp $((\mathrm{kJ} / \mathrm{mol}-\mathrm{K})$ & 0.1764756 \\
\hline
\end{tabular}

${ }^{1}$ Freeh output; $298.15 \mathrm{~K}, 0.1 \mathrm{MPa}$, unscaled

${ }^{4} \mathbf{A}$

Pentachlorophenol: $[($ Porphyrin $)(\mathrm{Fe}=\mathbf{O})(\mathrm{SH})]$ Prereactant Complex (ortho-oxidation)

\begin{tabular}{|c|c|c|c|}
\hline \multicolumn{2}{|c|}{$\mathrm{RI}-\mathrm{PBE} / \mathrm{TZVP}, \mathrm{SV}(\mathrm{P})$} & Cartesian & coordinates \\
\hline \multicolumn{4}{|c|}{53} \\
\hline \multicolumn{4}{|c|}{ Energy $=-5326.840703651$} \\
\hline $\mathrm{C}$ & -1.7438561 & 0.0928320 & 4.8294118 \\
\hline $\mathrm{N}$ & -1.1979421 & -0.6627398 & 3.8167262 \\
\hline $\mathrm{C}$ & -1.8223745 & -1.8870307 & 3.8735375 \\
\hline $\mathrm{C}$ & -2.7960536 & -1.9057601 & 4.9455981 \\
\hline $\mathrm{C}$ & -2.7329313 & -0.6775879 & 5.5542371 \\
\hline $\mathrm{Fe}$ & 0.2076493 & -0.0797777 & 2.4954012 \\
\hline 0 & -1.0385919 & 0.2611044 & 1.2603641 \\
\hline $\mathrm{C}$ & -1.5151233 & -2.9885150 & 3.0697130 \\
\hline $\mathrm{C}$ & -0.4796637 & -3.0300780 & 2.1334547 \\
\hline $\mathrm{I}$ & 0.3506601 & -1.9788155 & 1.8190970 \\
\hline
\end{tabular}




$\begin{array}{lrrr}\mathrm{C} & 1.2555647 & -2.4639464 & 0.9069298 \\ \mathrm{C} & 1.0048848 & -3.8656486 & 0.6459566 \\ \mathrm{C} & -0.0860574 & -4.2155678 & 1.4000911 \\ \mathrm{C} & 2.2262342 & -1.7034168 & 0.2490367 \\ \mathrm{C} & 2.3838809 & -0.3201517 & 0.3743046 \\ \mathrm{~N} & 1.6516579 & 0.4931514 & 1.2072856 \\ \mathrm{C} & 2.0486768 & 1.7812299 & 0.9331136 \\ \mathrm{C} & 3.0676908 & 1.7841248 & -0.0953517 \\ \mathrm{C} & 3.2885733 & 0.4720151 & -0.4317239 \\ \mathrm{C} & 1.5838221 & 2.9250853 & 1.5878316 \\ \mathrm{C} & 0.7003762 & 2.9204382 & 2.6709442 \\ \mathrm{~N} & 0.1063222 & 1.7994782 & 3.2075200 \\ \mathrm{C} & -0.6165327 & 2.2333261 & 4.2964235 \\ \mathrm{C} & -0.4615764 & 3.6629115 & 4.4642222 \\ \mathrm{C} & 0.3425801 & 4.0942682 & 3.4388827 \\ \mathrm{C} & -1.4512112 & 1.4350354 & 5.0828193 \\ \mathrm{~S} & 1.8254326 & -0.6018924 & 4.0312417 \\ \mathrm{H} & 2.5496829 & 0.5597040 & 3.9357140 \\ \mathrm{H} & 0.6834149 & 5.1142645 & 3.2163402 \\ \mathrm{H} & -0.9350900 & 4.2530487 & 5.2601755 \\ \mathrm{H} & -1.9706527 & 1.9183806 & 5.9242849 \\ \mathrm{H} & -3.3123846 & -0.3039947 & 6.4091210 \\ \mathrm{H} & -3.4305409 & -2.7656967 & 5.1985043 \\ \mathrm{H} & -2.0972091 & -3.9098038 & 3.2254387 \\ \mathrm{H} & -0.5898666 & -5.1888336 & 1.4698858 \\ \mathrm{H} & 1.5881385 & -4.4845947 & -0.0483060 \\ \mathrm{H} & 2.8700962 & -2.2233155 & -0.4760533 \\ \mathrm{H} & 3.9880529 & 0.0614188 & -1.1721423 \\ \mathrm{H} & 3.5505830 & 2.6864183 & -0.4936761 \\ \mathrm{H} & 1.9878615 & 3.8964442 & 1.2639472 \\ \mathrm{C} & -0.6114951 & 1.0435163 & -2.7270525 \\ \mathrm{C} & -0.7069546 & 1.4572482 & -4.0610386 \\ \mathrm{C} & -0.3643131 & -0.3518252 & -2.3610818 \\ \mathrm{C} & -0.1827088 & -1.2700845 & -3.4851646 \\ \mathrm{C} & -0.5336111 & 0.5170881 & -5.1151937 \\ \mathrm{C} & -0.2661870 & -0.8495626 & -4.8156593 \\ \mathrm{Cl} & -1.0252732 & 3.1158735 & -4.4416575 \\ \mathrm{Cl} & -0.6483149 & 1.0313704 & -6.7541644 \\ \mathrm{Cl} & 0.1544291 & -2.9118912 & -3.0875227 \\ \mathrm{Cl} & -0.8116203 & 2.1611302 & -1.4340127 \\ \mathrm{Cl} & -0.0436793 & -1.9784601 & -6.1091152 \\ \mathrm{O} & -0.2900458 & -0.7529048 & -1.1720692 \\ \mathrm{H} & -0.7563274 & -0.0396860 & 0.3385889\end{array}$

$\begin{array}{llllll}1551.74 & 1553.49 & 1572.50 & 1573.35 & 1602.86 & 1604.80 \\ 1659.05 & 2546.40 & 3054.18 & 3125.14 & 3125.23 & 3125.43 \\ 3131.39 & 3155.33 & 3155.48 & 3155.80 & 3157.46 & 3174.13\end{array}$

TURBOMOLE Energies and Thermochemistry ${ }^{1}$ RI-PBE/TZVP,SV(P)

$-5326.832526$

ZPE

RI-PBE/TZVP,SV(P) + ZPE

0.3340307

RI-PBE/TZVPP

B3-LYP/TZVP,SV(P)

$-5326.498495$

$\mathrm{ZPE}(\mathrm{kJ} / \mathrm{mol})$

$-5329.262083$

$-5328.50329$

In(qtrans)

877

In(qrot)

20.39

In(qvib)

chem.pot. $(\mathrm{kJ} / \mathrm{mol})$

energy $(\mathrm{kJ} / \mathrm{mol})$

704.54

966.77

entropy $(\mathrm{kJ} / \mathrm{mol} / \mathrm{K})$

0.88785

Cv (kJ/mol-K)

0.5532705

$\mathrm{Cp}((\mathrm{kJ} / \mathrm{mol}-\mathrm{K})$

$\mathrm{RI}-\mathrm{PBE} / \mathrm{TZVP}, \mathrm{SV}(\mathrm{P}) \quad<\mathrm{S}^{2}>$

0.5615848

3.77

$\mathrm{RI}-\mathrm{PBE} / \mathrm{TZVPP}<\mathrm{S}^{2}>$

3.77

B3 - LYP /TZVP, SV (P) $<\mathrm{S}^{2}>$

3.80

${ }^{1}$ Freeh output; $298.15 \mathrm{~K}, 0.1 \mathrm{MPa}$, unscaled

Vibrational frequencies

$\begin{array}{rrrrrr}0.00 & 0.00 & 0.00 & 0.00 & 0.00 & 0.00 \\ 5.42 & 11.65 & 19.53 & 33.79 & 40.29 & 43.25 \\ 48.37 & 51.20 & 69.64 & 77.24 & 86.01 & 93.39 \\ 104.29 & 111.90 & 134.03 & 144.56 & 149.89 & 169.50 \\ 178.11 & 199.43 & 202.73 & 207.30 & 208.76 & 211.38 \\ 217.56 & 235.41 & 254.58 & 269.44 & 270.88 & 282.29 \\ 289.75 & 293.27 & 296.87 & 304.00 & 309.27 & 311.00 \\ 324.22 & 341.47 & 347.44 & 354.32 & 359.26 & 360.22 \\ 365.71 & 372.01 & 400.25 & 401.91 & 415.79 & 418.53 \\ 421.02 & 422.04 & 456.73 & 466.40 & 541.81 & 552.63 \\ 635.76 & 644.35 & 645.82 & 649.05 & 654.08 & 655.92 \\ 669.66 & 672.24 & 674.45 & 690.98 & 692.64 & 695.94 \\ 696.58 & 717.26 & 724.32 & 727.52 & 734.82 & 736.48 \\ 762.61 & 769.51 & 769.73 & 770.60 & 776.53 & 779.67 \\ 784.28 & 784.75 & 799.18 & 838.67 & 847.82 & 849.14 \\ 857.27 & 860.79 & 886.43 & 891.68 & 891.98 & 893.76 \\ 977.85 & 986.13 & 995.81 & 1004.44 & 1005.17 & 1011.97 \\ 1018.51 & 1032.02 & 1033.46 & 1035.80 & 1041.60 & 1042.07 \\ 1043.18 & 1055.79 & 1096.82 & 1141.52 & 1148.92 & 1151.56 \\ 1183.98 & 1185.31 & 1236.52 & 1255.19 & 1263.55 & 1264.06 \\ 1298.60 & 1326.95 & 1328.33 & 1340.00 & 1364.34 & 1365.73 \\ 1378.66 & 1387.80 & 1389.45 & 1398.19 & 1412.90 & 1465.93 \\ 1467.57 & 1468.69 & 1500.39 & 1504.46 & 1520.33 & 1521.83\end{array}$


${ }^{4} \mathrm{~A}$

\section{Pentachlorophenol: [(Porphyrin $)(\mathrm{Fe}=\mathbf{O})(\mathrm{SH})]$} Transition State (ortho-oxidation)

\begin{tabular}{|c|c|c|c|}
\hline \multicolumn{2}{|c|}{ RI - PBE/TZVP, SV (P) } & Cartesian & coordinates \\
\hline 53 & & & \\
\hline & $y=-5326.79$ & 8603051 & \\
\hline $\mathrm{C}$ & -2.8444082 & -1.8656915 & 0.5161941 \\
\hline & -1.5363125 & -1.8087280 & 0.9309744 \\
\hline $\mathrm{C}$ & -1.0268256 & -3.0761813 & 0.7672227 \\
\hline $\mathrm{C}$ & -2.0407745 & -3.9600257 & 0.2255784 \\
\hline C & -3.1809987 & -3.2103199 & 0.0905407 \\
\hline $\mathrm{Fe}$ & -0.5331254 & -0.1718066 & 1.5517305 \\
\hline $\mathrm{O}$ & 0.1025674 & 0.0336081 & -0.0562945 \\
\hline $\mathrm{C}$ & 0.2544908 & -3.4841945 & 1.1449449 \\
\hline $\mathrm{C}$ & 1.1787898 & -2.6840910 & 1.8264736 \\
\hline $\mathrm{N}$ & 0.9986240 & -1.3563191 & 2.1371382 \\
\hline $\mathrm{C}$ & 2.0981243 & -0.9784562 & 2.8744481 \\
\hline $\mathrm{C}$ & 2.9980193 & -2.0999270 & 3.0397740 \\
\hline $\mathrm{C}$ & 2.4355645 & -3.1589332 & 2.3680791 \\
\hline $\mathrm{C}$ & 2.3676202 & 0.3212209 & 3.3142663 \\
\hline C & 1.5955145 & 1.4454321 & 3.0155528 \\
\hline $\mathrm{N}$ & 0.4183917 & 1.4396088 & 2.2974284 \\
\hline C & 0.0467255 & 2.7562828 & 2.1590702 \\
\hline $\mathrm{C}$ & 1.0169326 & 3.6226063 & 2.7967688 \\
\hline $\mathrm{C}$ & 1.9708797 & 2.8044236 & 3.3471714 \\
\hline C & -1.1376074 & 3.1963691 & 1.5607830 \\
\hline $\mathrm{C}$ & -2.1593196 & 2.3569728 & 1.1093618 \\
\hline $\mathrm{N}$ & -2.1127214 & 0.9800662 & 1.1085930 \\
\hline $\mathrm{C}$ & -3.3479151 & 0.5556810 & 0.6707858 \\
\hline $\mathrm{C}$ & -4.2037895 & 1.6937358 & 0.4037858 \\
\hline $\mathrm{C}$ & -3.4565963 & 2.8160667 & 0.6567588 \\
\hline $\mathrm{C}$ & -3.7110207 & -0.7721286 & 0.4345273 \\
\hline S & -1.3548676 & -0.5107265 & 3.7214685 \\
\hline $\mathrm{H}$ & -1.8376849 & 0.7554414 & 3.9 \\
\hline $\mathrm{H}$ & -3.7491759 & 3.8705678 & 0.5644804 \\
\hline $\mathrm{H}$ & -5.2403839 & 1.6254250 & 0.0477310 \\
\hline $\mathrm{H}$ & -4.7369586 & -0.9618116 & 0.0827935 \\
\hline $\mathrm{H}$ & -4.1667150 & -3.5204041 & -0.2812593 \\
\hline $\mathrm{H}$ & -1.8870075 & -5.0237784 & 0.0001966 \\
\hline $\mathrm{H}$ & 0.5320024 & -4.5316291 & 0.9532338 \\
\hline $\mathrm{H}$ & 2.8144852 & -4.1846019 & 2.2641531 \\
\hline $\mathrm{H}$ & 3.9458855 & -2.0657134 & 3.5935297 \\
\hline $\mathrm{H}$ & 3.2930044 & 0.4799506 & 3.8892163 \\
\hline $\mathrm{H}$ & 2.8661451 & 3.0828649 & 3.9190499 \\
\hline $\mathrm{H}$ & 0.9521411 & 4.7184912 & 2.8266309 \\
\hline $\mathrm{H}$ & -1.3110862 & 4.2821722 & 1.5103103 \\
\hline $\mathrm{C}$ & 1.5755701 & 0.2754731 & -1.2306040 \\
\hline C & 1.1402655 & 1.4261513 & -1.9707576 \\
\hline $\mathrm{C}$ & 1.4928202 & -1.0261811 & -1.8601194 \\
\hline C & 0.7583875 & -1.1738390 & -3.0475851 \\
\hline $\mathrm{C}$ & 0.4066015 & 1.2592091 & -3.1533520 \\
\hline $\mathrm{C}$ & 0.2191539 & -0.0417282 & -3.7002963 \\
\hline $\mathrm{Cl}$ & 1.4053391 & 2.9939169 & -1.3048603 \\
\hline $\mathrm{Cl}$ & -0.2373127 & 2.6371253 & -3.9773502 \\
\hline $\mathrm{Cl}$ & 0.5664602 & -2.7630154 & -3.7044537 \\
\hline $\mathrm{Cl}$ & 2.9376056 & 0.4613282 & -0.1233877 \\
\hline $\mathrm{Cl}$ & -0.6739189 & -0.2422706 & -5.1600078 \\
\hline 0 & 2.0404818 & -2.1038212 & -1.2816846 \\
\hline $\mathrm{H}$ & 2.4843709 & -1.8078246 & -0.4446717 \\
\hline
\end{tabular}

$\begin{array}{rrrrrr}1498.70 & 0.00 & 0.00 & 0.00 & 0.00 & 0.00 \\ 0.00 & 9.25 & 21.08 & 29.27 & 41.96 & 45.31 \\ 50.98 & 57.11 & 67.56 & 78.19 & 85.10 & 91.27 \\ 99.84 & 116.64 & 125.04 & 133.14 & 147.26 & 153.29 \\ 194.87 & 196.55 & 200.14 & 209.18 & 210.14 & 213.24 \\ 215.25 & 234.20 & 240.43 & 248.88 & 269.91 & 273.07 \\ 282.50 & 290.59 & 293.08 & 298.62 & 318.66 & 322.57 \\ 329.01 & 338.34 & 343.82 & 348.81 & 359.91 & 360.48 \\ 363.97 & 370.61 & 411.71 & 412.68 & 419.97 & 421.27 \\ 421.74 & 421.95 & 451.20 & 459.14 & 464.67 & 471.02 \\ 587.55 & 597.61 & 605.98 & 637.04 & 652.02 & 655.40 \\ 656.77 & 670.99 & 675.04 & 679.14 & 693.93 & 694.31 \\ 695.91 & 698.68 & 717.52 & 724.34 & 734.26 & 735.85 \\ 753.90 & 763.05 & 770.22 & 770.57 & 776.45 & 781.56 \\ 785.04 & 785.70 & 799.66 & 840.49 & 848.42 & 850.01 \\ 856.95 & 858.00 & 885.85 & 890.84 & 891.80 & 893.57 \\ 976.28 & 994.49 & 1003.62 & 1003.90 & 1010.23 & 1016.06 \\ 1030.78 & 1031.92 & 1034.90 & 1040.26 & 1041.02 & 1042.04 \\ 1054.92 & 1080.68 & 1140.08 & 1147.23 & 1149.15 & 1182.27 \\ 1183.44 & 1212.75 & 1247.16 & 1261.35 & 1263.23 & 1313.08 \\ 1324.34 & 1327.85 & 1329.92 & 1338.64 & 1349.52 & 1363.81 \\ 1376.69 & 1384.72 & 1389.94 & 1393.09 & 1424.53 & 1464.69 \\ 1468.16 & 1470.10 & 1481.04 & 1501.14 & 1521.35 & 1551.52 \\ 1552.48 & 1555.60 & 1574.94 & 1593.02 & 1601.81 & 1607.09 \\ 1655.62 & 2548.31 & 3121.89 & 3122.34 & 3124.77 & 3128.24 \\ 3153.90 & 3154.77 & 3155.18 & 3155.41 & 3172.61 & 3173.76 \\ 3174.12 & 3174.41 & 3386.80 & & & \end{array}$

TURBOMOLE Energies and Thermochemistry ${ }^{1}$

\begin{tabular}{|c|c|}
\hline RI-PBE/TZVP,SV(P) & -5326.795977 \\
\hline ZPE & 0.3343481 \\
\hline RI-PBE/TZVP,SV(P) + ZPE & -5326.461628 \\
\hline RI-PBE/TZVPP & -5329.226984 \\
\hline B3-LYP/TZVP,SV(P) & -5328.440118 \\
\hline ZPE (kJ/mol) & 877.8 \\
\hline In(qtrans) & 20.39 \\
\hline In(qrot) & 17.45 \\
\hline $\ln (q v i b)$ & 34.35 \\
\hline chem.pot. (kJ/mol) & 698.88 \\
\hline energy $(\mathrm{kJ} / \mathrm{mol})$ & 969.31 \\
\hline entropy $(\mathrm{kJ} / \mathrm{mol} / \mathrm{K})$ & 0.91533 \\
\hline $\mathrm{Cv}(\mathrm{kJ} / \mathrm{mol}-\mathrm{K})$ & 0.5586086 \\
\hline $\mathrm{Cp}((\mathrm{kJ} / \mathrm{mol}-\mathrm{K})$ & 0.5669229 \\
\hline $\mathrm{RI}-\mathrm{PBE} / \mathrm{TZVP}, \mathrm{SV}(\mathrm{P}) \quad<\mathrm{S}^{2}>$ & 3.77 \\
\hline $\mathrm{RI}-\mathrm{PBE} / \mathrm{TZVPP}<\mathrm{S}^{2}>$ & 3.77 \\
\hline B3 - LYP / TZVP, SV (P) $\quad\left\langle\mathrm{S}^{2}>\right.$ & 4.98 \\
\hline
\end{tabular}

${ }^{1}$ Freeh output; $298.15 \mathrm{~K}, 0.1 \mathrm{MPa}$, unscaled 
${ }^{4} \mathrm{~A}$

\section{Pentachlorophenol:[(Porphyrin $)(\mathrm{Fe}=\mathrm{O})(\mathrm{SH})]$ Product Complex (ortho-oxidation)}

RI-PBE/TZVP, SV (P) Cartesian coordinates

$$
53
$$$$
\text { Energy }=-5326.853201940
$$

$\begin{array}{lrrr}\mathrm{C} & -2.1941459 & 1.3511394 & 3.1472885 \\ \mathrm{~N} & -1.6157612 & 0.1597979 & 2.7395303 \\ \mathrm{C} & -2.5598033 & -0.8189105 & 2.9924258 \\ \mathrm{C} & -3.7493558 & -0.2343836 & 3.5682482 \\ \mathrm{C} & -3.5202360 & 1.1154986 & 3.6674244 \\ \mathrm{Fe} & 0.1951243 & -0.0797828 & 1.9231855 \\ \mathrm{O} & -0.6086992 & 0.1894091 & 0.1088146 \\ \mathrm{C} & -2.4107151 & -2.1850053 & 2.7480222 \\ \mathrm{C} & -1.2675931 & -2.7776733 & 2.2142363 \\ \mathrm{~N} & -0.1486474 & -2.0921429 & 1.7694291 \\ \mathrm{C} & 0.7737607 & -3.0674928 & 1.4193304 \\ \mathrm{C} & 0.2059517 & -4.3813088 & 1.5983499 \\ \mathrm{C} & -1.0660191 & -4.2015152 & 2.0861740 \\ \mathrm{C} & 2.0544757 & -2.8108013 & 0.9369788 \\ \mathrm{C} & 2.5982900 & -1.5370884 & 0.7756476 \\ \mathrm{~N} & 2.0011119 & -0.3419772 & 1.1284804 \\ \mathrm{C} & 2.9029587 & 0.6472018 & 0.7677714 \\ \mathrm{C} & 4.0724024 & 0.0663601 & 0.1619345 \\ \mathrm{C} & 3.8844116 & -1.2961640 & 0.1667449 \\ \mathrm{C} & 2.7319142 & 2.0173261 & 0.9649384 \\ \mathrm{C} & 1.6139505 & 2.5975529 & 1.5598301 \\ \mathrm{~N} & 0.4998639 & 1.9143559 & 1.9936387 \\ \mathrm{C} & -0.3498878 & 2.8646680 & 2.5095271 \\ \mathrm{C} & 0.2380028 & 4.1833892 & 2.3961769 \\ \mathrm{C} & 1.4616019 & 4.0172816 & 1.7995834 \\ \mathrm{C} & -1.6086356 & 2.6123836 & 3.0559937 \\ \mathrm{~S} & 0.9802647 & -0.3313859 & 3.9722083 \\ \mathrm{H} & 2.3103453 & -0.4927650 & 3.6703549 \\ \mathrm{H} & 2.2091714 & 4.7774664 & 1.5370951 \\ \mathrm{H} & -0.2430248 & 5.1113985 & 2.7331353 \\ \mathrm{H} & -2.1879977 & 3.4715831 & 3.4262387 \\ \mathrm{H} & -4.1869048 & 1.8992560 & 4.0505888 \\ \mathrm{H} & -4.6451736 & -0.8018373 & 3.8536996 \\ \mathrm{H} & -3.2442101 & -2.8458348 & 3.0297571 \\ \mathrm{H} & -1.8127994 & -4.9621882 & 2.3499796 \\ \mathrm{H} & 0.7261930 & -5.3208521 & 1.3698166 \\ \mathrm{H} & 2.6708514 & -3.6695474 & 0.6330039 \\ \mathrm{H} & 4.5445992 & -2.0817365 & -0.2216132 \\ \mathrm{H} & 4.9206855 & 0.6399293 & -0.2334611 \\ \mathrm{H} & 3.5428634 & 2.6840602 & 0.6358788 \\ \mathrm{C} & -0.5648989 & 0.1958489 & -1.1733945 \\ \mathrm{C} & -0.5318820 & 1.3797484 & -1.9784300 \\ \mathrm{C} & -0.6522079 & -1.0898165 & -1.8659419 \\ \mathrm{C} & -0.7911778 & -1.1455862 & -3.2807339 \\ \mathrm{C} & -0.6481883 & 1.3049235 & -3.3795819 \\ \mathrm{C} & -0.7664876 & 0.0453945 & -4.0340041 \\ \mathrm{C} 1 & -0.4326766 & 2.9056103 & -1.1806062 \\ \mathrm{Cl} & -0.6243424 & 2.7513675 & -4.3223758 \\ \mathrm{Cl} & -1.0069557 & -2.6814031 & -4.0263665 \\ \mathrm{Cl} & 2.0495829 & -0.9946463 & -2.3869229 \\ \mathrm{Cl} & -0.8953511 & -0.0269171 & -5.7502763 \\ \mathrm{O} & -0.8051891 & -2.1795839 & -1.1311342 \\ \mathrm{H} & -0.6833739 & -1.9144463 & -0.1683894\end{array}$

$\begin{array}{rrrrrr}0.00 & 0.00 & 0.00 & 0.00 & 0.00 & 0.00 \\ 16.82 & 26.72 & 34.81 & 47.56 & 52.80 & 55.36 \\ 59.66 & 69.53 & 76.22 & 81.80 & 83.39 & 94.01 \\ 101.33 & 108.93 & 121.79 & 131.36 & 143.72 & 149.63 \\ 176.17 & 183.21 & 200.67 & 203.63 & 212.56 & 215.31 \\ 216.95 & 227.88 & 253.80 & 255.51 & 270.01 & 273.29 \\ 277.96 & 281.56 & 290.64 & 292.75 & 300.76 & 317.29 \\ 336.46 & 339.54 & 346.35 & 356.60 & 358.59 & 360.52 \\ 370.93 & 385.28 & 401.71 & 405.23 & 412.61 & 417.48 \\ 422.66 & 424.07 & 436.19 & 453.70 & 510.82 & 535.09 \\ 551.12 & 629.15 & 647.36 & 650.47 & 657.42 & 665.67 \\ 670.04 & 677.71 & 679.83 & 692.00 & 694.99 & 698.44 \\ 715.70 & 721.29 & 730.84 & 731.83 & 737.57 & 761.20 \\ 763.96 & 772.30 & 772.85 & 781.44 & 781.94 & 784.15 \\ 787.54 & 798.56 & 812.83 & 837.08 & 845.86 & 847.80 \\ 855.15 & 888.29 & 893.44 & 896.05 & 899.05 & 968.85 \\ 990.10 & 996.73 & 998.38 & 1002.39 & 1012.07 & 1016.66 \\ 1031.67 & 1032.68 & 1037.09 & 1041.61 & 1042.28 & 1044.21 \\ 1051.69 & 1137.68 & 1139.47 & 1146.09 & 1181.24 & 1183.72 \\ 1202.02 & 1246.75 & 1259.28 & 1263.03 & 1301.65 & 1324.94 \\ 1328.33 & 1330.74 & 1342.91 & 1350.77 & 1362.89 & 1370.27 \\ 1372.65 & 1379.83 & 1388.15 & 1429.02 & 1450.27 & 1464.93 \\ 1468.46 & 1474.23 & 1478.62 & 1510.98 & 1517.42 & 1532.75 \\ 1549.60 & 1557.04 & 1578.32 & 1606.17 & 1610.75 & 1625.27 \\ 1664.75 & 2533.40 & 3126.96 & 3127.37 & 3130.19 & 3134.11 \\ 3150.11 & 3156.43 & 3157.23 & 3158.17 & 3166.16 & 3175.49 \\ 3175.96 & 3176.49 & 3185.49 & & & \end{array}$

TURBOMOLE Energies and Thermochemistry ${ }^{1}$ RI-PBE/TZVP,SV(P)

$-5326.845273$

ZPE

0.3350555

RI-PBE/TZVP,SV(P) + ZPE

$-5326.510217$

RI-PBE/TZVPP

$-5329.273235$

B3-LYP/TZVP,SV(P)

$-5328.521261$

ZPE $(\mathrm{kJ} / \mathrm{mol})$

879.7

In(qtrans)

20.39

$\ln$ (qrot)

17.55

In(qvib)

34.1

chem.pot. $(\mathrm{kJ} / \mathrm{mol})$

701.11

energy $(\mathrm{kJ} / \mathrm{mol})$

971.79

entropy $(\mathrm{kJ} / \mathrm{mol} / \mathrm{K})$

0.91619

$\mathrm{Cv}(\mathrm{kJ} / \mathrm{mol}-\mathrm{K})$

0.5581547

$\mathrm{Cp}((\mathrm{kJ} / \mathrm{mol}-\mathrm{K})$

RI - PBE/TZVP, SV (P) $<\mathrm{S}^{2}>$

0.566469

3.78

$\mathrm{RI}-\mathrm{PBE} / \mathrm{TZVPP}<\mathrm{S}^{2}>$

3.78

B3 - LYP/TZVP , SV (P) $<\mathrm{S}^{2}>$

3.90

${ }^{1}$ Freeh output; $298.15 \mathrm{~K}, 0.1 \mathrm{MPa}$, unscaled 
Pentachlorophenol: [(Porphyrin $)(\mathrm{Fe}=\mathbf{O})(\mathrm{SH})]$ Prereactant Complex (ipso-addition)

\begin{tabular}{|c|c|c|c|}
\hline \multicolumn{2}{|c|}{$\mathrm{RI}-\mathrm{PBE} / \mathrm{TZVP}, \mathrm{SV}(\mathrm{P})$} & Cartesian & coordinates \\
\hline & & & \\
\hline Ene & $y=-5326.83$ & 7410457 & \\
\hline $\mathrm{C}$ & -2.4231422 & -0.4766971 & 4.1670646 \\
\hline & -1.1392334 & -0.8249798 & 3.8034731 \\
\hline & -0.8102547 & -1.9196080 & 4.5672408 \\
\hline & -1.9018300 & -2.2661576 & 5.4518529 \\
\hline C & -2.9088884 & -1.3699048 & 5.1977449 \\
\hline $\mathrm{Fe}$ & -0.0186314 & 0.0160564 & 2.3324885 \\
\hline 0 & -0.8324149 & -0.8151705 & 1.1484420 \\
\hline $\mathrm{C}$ & 0.3961332 & -2.6184318 & 4.4828577 \\
\hline C & 1.4341531 & -2.3187982 & 3.5995487 \\
\hline & 1.4404924 & -1.2851831 & 2.6844893 \\
\hline C & 2.6612531 & -1.3568581 & 2.0433378 \\
\hline C & 3.4350179 & -2.4662466 & 2.5558229 \\
\hline C & 2.6727284 & -3.0630184 & 3.5270597 \\
\hline $\mathrm{C}$ & 3.1120037 & -0.4809176 & 1.0567849 \\
\hline $\mathrm{C}$ & 2.3948743 & 0.6162127 & 0.5756650 \\
\hline $\mathrm{N}$ & 1.1415718 & 0.9918426 & 1.0039446 \\
\hline $\mathrm{C}$ & 0.8006564 & 2.0990296 & 0.2528610 \\
\hline $\mathrm{C}$ & 1.8726518 & 2.4346467 & -0.6595054 \\
\hline C & 2.8662366 & 1.5100726 & -0.4587833 \\
\hline C & -0.4071770 & 2.7994659 & 0.3204133 \\
\hline C & -1.4773238 & 2.4844560 & 1.1580827 \\
\hline $\mathrm{N}$ & -1.4866587 & 1.4523927 & 31825 \\
\hline C & -2.7334440 & 1.4670425 & 2.6562354 \\
\hline C & -3.5251485 & 2.5533879 & 2.11 \\
\hline C & -2.7430271 & 3.1876418 & 1.1846884 \\
\hline C & -3.1725413 & 0.5746487 & 3.6336003 \\
\hline S & 0.5740375 & 1.4992536 & 4.0340369 \\
\hline $\mathrm{H}$ & 1.0279490 & 2.5343212 & 3.2553441 \\
\hline $\mathrm{H}$ & -2.9896454 & 4.0509446 & 0.5529017 \\
\hline $\mathrm{H}$ & -4.5540786 & 2.7868314 & 2.4203357 \\
\hline $\mathrm{H}$ & -4.1945397 & 0.7105721 & 4.0195238 \\
\hline $\mathrm{H}$ & -3.9027901 & -1.3047252 & 5.6601292 \\
\hline $\mathrm{H}$ & -1.8866589 & -3.0998782 & 6.1662377 \\
\hline $\mathrm{H}$ & 0.5350762 & -3.4782679 & 5.1552369 \\
\hline $\mathrm{H}$ & 2.9128435 & -3.9334996 & 8074 \\
\hline $\mathrm{H}$ & 4.440 & -2.73 & 2.2076306 \\
\hline $\mathrm{H}$ & 4.1063417 & -0.6693387 & 11635 \\
\hline $\mathrm{H}$ & 3.8347661 & 1.4205738 & -0.96 \\
\hline $\mathrm{H}$ & 1.84 & 3.2710492 & -1.3702056 \\
\hline $\mathrm{H}$ & -0.5359284 & 3.6459817 & -0.3696898 \\
\hline C & -0.6132504 & 0.0546586 & -2.1863784 \\
\hline $\mathrm{C}$ & -0.7336674 & 1.0841176 & -3.1614918 \\
\hline C & 0.4484251 & -0.8766356 & -2.3500087 \\
\hline $\mathrm{C}$ & 1.3696462 & -0.7637050 & -3.4125779 \\
\hline C & 0.1709406 & 1.1926217 & -4.2363160 \\
\hline $\mathrm{C}$ & 1.2357188 & 0.2708778 & -4.3645241 \\
\hline $\mathrm{Cl}$ & -2.0240595 & 2.2264764 & -2.9755859 \\
\hline $\mathrm{Cl}$ & -0.0087077 & 2.4689454 & -5.3920242 \\
\hline $\mathrm{Cl}$ & 2.6659505 & -1.9037268 & -3.5524309 \\
\hline $\mathrm{Cl}$ & 0.5839213 & -2.1786902 & -1.2109366 \\
\hline $\mathrm{Cl}$ & 2.3623961 & 0.4099099 & -5.6723470 \\
\hline 0 & -1.5101598 & 0.0113855 & -1.2062962 \\
\hline $\mathrm{H}$ & -1.1941031 & -0.4724682 & -0.3557331 \\
\hline
\end{tabular}

$\begin{array}{rrrrrr}14.80 & 0.00 & 0.00 & 0.00 & 0.00 & 0.00 \\ 0.00 & 11.37 & 17.31 & 21.30 & 38.03 & 46.39 \\ 52.45 & 57.79 & 70.23 & 77.33 & 96.15 & 99.89 \\ 104.07 & 108.69 & 143.64 & 147.42 & 165.21 & 168.11 \\ 180.85 & 202.21 & 205.67 & 206.13 & 208.85 & 216.35 \\ 220.24 & 230.12 & 237.33 & 270.22 & 272.08 & 275.97 \\ 280.84 & 290.21 & 291.92 & 306.36 & 313.83 & 318.05 \\ 331.51 & 332.02 & 345.43 & 354.77 & 358.58 & 361.69 \\ 367.91 & 371.86 & 396.65 & 403.52 & 417.23 & 418.27 \\ 420.23 & 422.12 & 458.33 & 459.75 & 543.06 & 555.71 \\ 605.30 & 633.44 & 639.53 & 649.53 & 656.11 & 656.46 \\ 671.78 & 675.97 & 691.99 & 694.09 & 698.46 & 705.48 \\ 713.23 & 723.14 & 732.68 & 734.56 & 739.24 & 759.97 \\ 765.16 & 773.42 & 774.03 & 779.74 & 780.44 & 784.82 \\ 784.95 & 798.55 & 806.39 & 844.33 & 853.87 & 854.66 \\ 862.99 & 866.34 & 889.54 & 895.02 & 895.36 & 897.38 \\ 980.56 & 992.79 & 1001.84 & 1003.07 & 1011.13 & 1015.98 \\ 1032.53 & 1032.86 & 1036.78 & 1041.91 & 1042.02 & 1043.45 \\ 1053.63 & 1113.55 & 1139.75 & 1148.80 & 1149.88 & 1156.03 \\ 1183.79 & 1184.81 & 1262.64 & 1263.77 & 1275.77 & 1300.64 \\ 1328.84 & 1330.02 & 1330.62 & 1342.00 & 1363.26 & 1363.59 \\ 1375.07 & 1379.46 & 1388.93 & 1390.49 & 1447.03 & 1467.89 \\ 1471.55 & 1472.13 & 1503.92 & 1520.63 & 1524.54 & 1556.13 \\ 1557.18 & 1566.59 & 1577.63 & 1594.25 & 1609.12 & 1611.82 \\ 1664.65 & 2546.99 & 2798.92 & 3124.78 & 3127.48 & 3127.80 \\ 3134.35 & 3157.15 & 3157.36 & 3157.72 & 3159.75 & 3175.81 \\ 3176.08 & 3176.42 & 3178.95 & & & \end{array}$

TURBOMOLE Energies and Thermochemistry ${ }^{1}$

\begin{tabular}{|c|c|}
\hline RI-PBE/TZVP,SV(P) & -5326.83741 \\
\hline ZPE & 0.3348088 \\
\hline RI-PBE/TZVP,SV(P) + ZPE & -5326.500435 \\
\hline RI-PBE/TZVPP & -5329.26769 \\
\hline B3-LYP/TZVP,SV(P) & -5328.505429 \\
\hline ZPE (kJ/mol) & 879 \\
\hline $\ln ($ qtrans $)$ & 20.39 \\
\hline $\ln ($ qrot $)$ & 17.67 \\
\hline In(qvib) & 33.46 \\
\hline chem.pot. (kJ/mol) & 701.77 \\
\hline energy $(\mathrm{kJ} / \mathrm{mol})$ & 969.48 \\
\hline entropy (kJ/mol/K) & 0.90622 \\
\hline $\mathrm{Cv}(\mathrm{kJ} / \mathrm{mol}-\mathrm{K})$ & 0.5538065 \\
\hline $\mathrm{Cp}((\mathrm{kJ} / \mathrm{mol}-\mathrm{K})$ & 0.5621208 \\
\hline $\mathrm{RI}-\mathrm{PBE} / \mathrm{TZVP}, \mathrm{SV}(\mathrm{P}) \quad<\mathrm{S}^{2}>$ & 1.18 \\
\hline $\mathrm{RI}-\mathrm{PBE} / \mathrm{TZVPP} \quad<\mathrm{S}^{2}>$ & 1.16 \\
\hline B3 - LYP / TZVP , SV (P) $<\mathrm{S}^{2}>$ & 1.66 \\
\hline
\end{tabular}

${ }^{1}$ Freeh output; $298.15 \mathrm{~K}, 0.1 \mathrm{MPa}$, unscaled 
${ }^{2} \mathbf{A}$

Pentachlorophenol: [(Porphyrin $)(\mathrm{Fe}=\mathbf{O})(\mathrm{SH})]$ Transition State (ipso-addition)

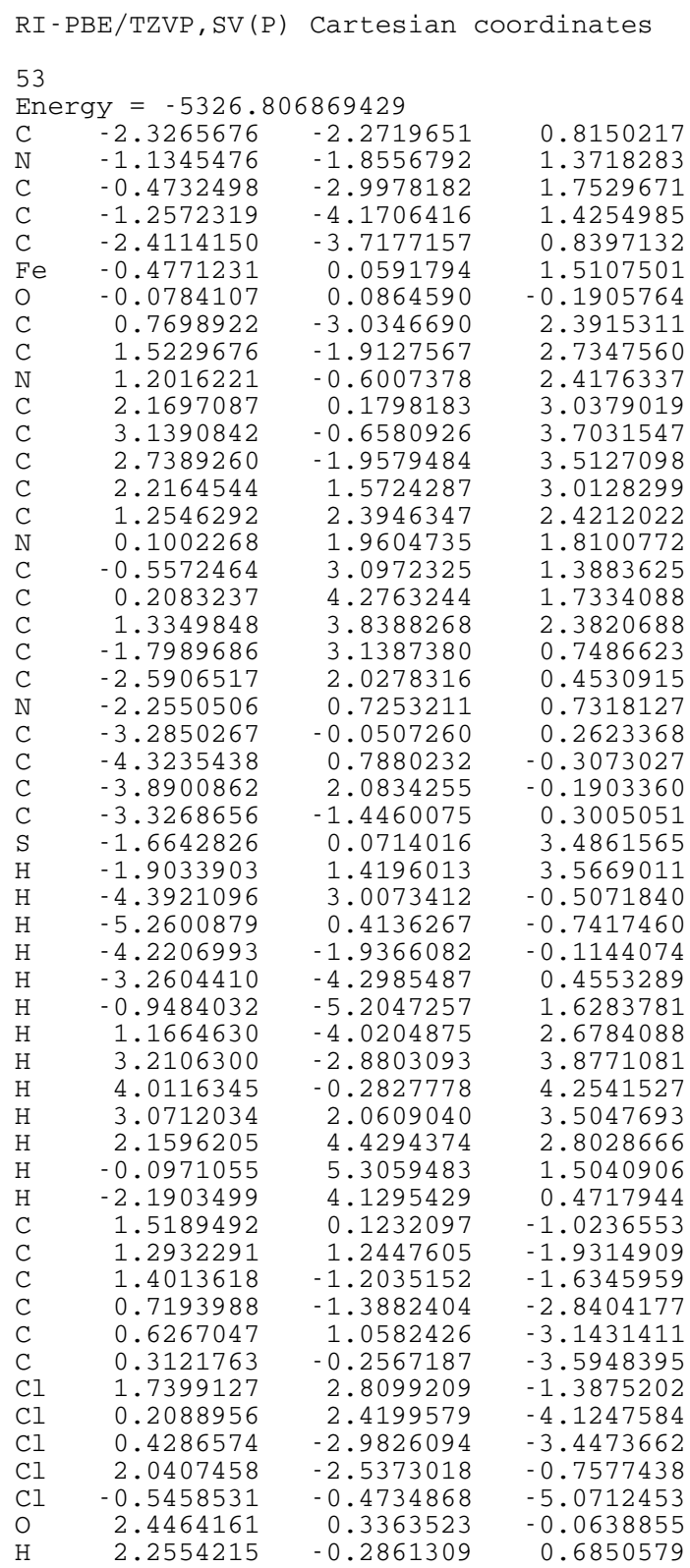

$\begin{array}{rrrrrr}1503.85 & 0.00 & 0.00 & 0.00 & 0.00 & 0.00 \\ 0.00 & 12.68 & 23.47 & 31.15 & 44.71 & 49.53 \\ 57.30 & 59.75 & 72.35 & 78.17 & 86.43 & 89.03 \\ 103.48 & 108.18 & 123.36 & 143.78 & 151.44 & 155.10 \\ 179.26 & 198.43 & 203.58 & 207.24 & 207.94 & 215.32 \\ 216.62 & 231.03 & 254.67 & 263.57 & 269.24 & 273.13 \\ 279.95 & 281.17 & 286.20 & 294.48 & 312.48 & 319.72 \\ 327.34 & 331.88 & 344.00 & 349.75 & 356.73 & 361.75 \\ 363.52 & 368.10 & 394.35 & 395.51 & 413.45 & 417.69 \\ 419.62 & 421.60 & 426.18 & 458.67 & 472.56 & 527.29 \\ 561.48 & 609.73 & 626.95 & 637.65 & 650.39 & 656.71 \\ 658.08 & 673.93 & 681.03 & 692.12 & 695.21 & 696.80 \\ 698.57 & 709.94 & 716.65 & 723.02 & 733.96 & 735.29 \\ 763.21 & 766.45 & 771.13 & 772.26 & 778.85 & 782.67 \\ 784.88 & 786.15 & 798.70 & 839.26 & 848.08 & 849.14 \\ 856.48 & 865.81 & 887.38 & 892.42 & 892.95 & 895.79 \\ 960.35 & 989.63 & 998.42 & 1002.31 & 1010.76 & 1013.48 \\ 1031.08 & 1032.57 & 1036.43 & 1040.48 & 1041.91 & 1042.22 \\ 1052.95 & 1101.62 & 1138.48 & 1146.72 & 1148.61 & 1182.11 \\ 1182.98 & 1187.15 & 1252.86 & 1260.49 & 1262.16 & 1296.90 \\ 1323.15 & 1328.94 & 1331.76 & 1344.57 & 1352.56 & 1363.53 \\ 1369.88 & 1381.43 & 1390.72 & 1394.10 & 1396.19 & 1444.34 \\ 1469.58 & 1471.79 & 1473.02 & 1505.37 & 1515.66 & 1523.49 \\ 1556.59 & 1558.11 & 1578.98 & 1606.20 & 1607.68 & 1618.56 \\ 1660.44 & 2549.23 & 3122.77 & 3124.29 & 3124.38 & 3125.58 \\ 3154.86 & 3155.18 & 3155.31 & 3155.75 & 3173.95 & 3174.21 \\ 3174.32 & 3174.48 & 3372.36 & & & \end{array}$

TURBOMOLE Energies and Thermochemistry ${ }^{1}$ RI-PBE/TZVP,SV(P)

$-5326.806869$

ZPE

0.334296

RI-PBE/TZVP,SV(P) + ZPE

RI-PBE/TZVPP

$-5326.472573$

B3-LYP/TZVP,SV(P)

$-5329.235559$

$\mathrm{ZPE}(\mathrm{kJ} / \mathrm{mol})$

$-5328.468964$

$\ln$ (qtrans)

877.7

In(qrot)

20.39

17.44

In(qvib)

32.99

chem.pot. $(\mathrm{kJ} / \mathrm{mol})$

702.12

energy $(\mathrm{kJ} / \mathrm{mol})$

969.09

entropy $(\mathrm{kJ} / \mathrm{mol} / \mathrm{K})$

0.90374

$\mathrm{Cv}(\mathrm{kJ} / \mathrm{mol}-\mathrm{K})$

0.5586185

$\mathrm{Cp}((\mathrm{kJ} / \mathrm{mol}-\mathrm{K})$

0.5669328

$\mathrm{RI}-\mathrm{PBE} / \mathrm{TZVP}, \mathrm{SV}(\mathrm{P}) \quad<\mathrm{S}^{2}>$

1.01

$\mathrm{RI}-\mathrm{PBE} / \mathrm{TZVPP}\left\langle\mathrm{S}^{2}\right\rangle \quad 0.98$

B3 - LYP / TZVP , SV (P) $\left\langle\mathrm{S}^{2}>\right.$

1.56

${ }^{1}$ Freeh output; $298.15 \mathrm{~K}, 0.1 \mathrm{MPa}$, unscaled 
${ }^{2} \mathbf{A}$

\section{Pentachlorophenol: [(Porphyrin $)(\mathrm{Fe}=\mathbf{O})(\mathrm{SH})]$ $\sigma$-Complex (ipso-addition)}

RI-PBE/TZVP, SV (P) Cartesian coordinates

$$
53
$$$$
\text { Energy }=-5326.823287292
$$

$\begin{array}{lrrr}\mathrm{C} & -2.9408371 & -1.4142259 & 0.6524773 \\ \mathrm{~N} & -1.6872417 & -1.4978320 & 1.2228197 \\ \mathrm{C} & -1.4586224 & -2.8447828 & 1.4353047 \\ \mathrm{C} & -2.5939760 & -3.6232805 & 0.9898782 \\ \mathrm{C} & -3.5106382 & -2.7342573 & 0.4889209 \\ \mathrm{Fe} & -0.4491799 & 0.0074491 & 1.6630241 \\ \mathrm{O} & 0.2512310 & 0.1024646 & -0.1037777 \\ \mathrm{C} & -0.2908795 & -3.4008283 & 1.9551983 \\ \mathrm{C} & 0.8583619 & -2.6806070 & 2.2947374 \\ \mathrm{~N} & 0.9950290 & -1.3147251 & 2.2007517 \\ \mathrm{C} & 2.2871387 & -1.0320415 & 2.5909723 \\ \mathrm{C} & 2.9925203 & -2.2550062 & 2.9138526 \\ \mathrm{C} & 2.0952826 & -3.2807763 & 2.7445574 \\ \mathrm{C} & 2.8304033 & 0.2510190 & 2.7155814 \\ \mathrm{C} & 2.1149319 & 1.4391627 & 2.5472315 \\ \mathrm{~N} & 0.7944070 & 1.5287447 & 2.1697450 \\ \mathrm{C} & 0.4912212 & 2.8690622 & 2.1614340 \\ \mathrm{C} & 1.6440660 & 3.6503760 & 2.5595801 \\ \mathrm{C} & 2.6613552 & 2.7588642 & 2.7873825 \\ \mathrm{C} & -0.7331686 & 3.4197312 & 1.7716653 \\ \mathrm{C} & -1.8167520 & 2.6951469 & 1.2770697 \\ \mathrm{~N} & -1.8748639 & 1.3223352 & 1.1442266 \\ \mathrm{C} & -3.1005702 & 1.0482348 & 0.5807721 \\ \mathrm{C} & -3.8334445 & 2.2745311 & 0.3419592 \\ \mathrm{C} & -3.0385312 & 3.2993085 & 0.7875288 \\ \mathrm{C} & -3.5974920 & -0.2293231 & 0.3179687 \\ \mathrm{~S} & -1.1898513 & -0.0299944 & 3.7616521 \\ \mathrm{H} & -0.0220719 & 0.2811409 & 4.4131539 \\ \mathrm{H} & -3.2433054 & 4.3782383 & 0.7853938 \\ \mathrm{H} & -4.8353792 & 2.3255235 & -0.1045364 \\ \mathrm{H} & -4.5981234 & -0.3079715 & -0.1331941 \\ \mathrm{H} & -4.4972390 & -2.9376111 & 0.0516502 \\ \mathrm{H} & -2.6594306 & -4.7179542 & 1.0479563 \\ \mathrm{H} & -0.2576308 & -4.4947501 & 2.0714714 \\ \mathrm{H} & 2.2475439 & -4.3571647 & 2.9009324 \\ \mathrm{H} & 4.0374864 & -2.3040894 & 3.2478310 \\ \mathrm{H} & 3.8842680 & 0.3303513 & 3.0221304 \\ \mathrm{H} & 3.6957684 & 2.9601405 & 3.0963632 \\ \mathrm{H} & 1.6598135 & 4.7457623 & 2.6362617 \\ \mathrm{H} & -0.8311663 & 4.5155687 & 1.8080970 \\ \mathrm{C} & 1.2495020 & 0.0622156 & -1.0286260 \\ \mathrm{C} & 1.0583814 & 1.2806868 & -1.9392283 \\ \mathrm{C} & 1.0664034 & -1.2313854 & -1.8362402 \\ \mathrm{C} & 0.5078004 & -1.2781725 & -3.1045055 \\ \mathrm{C} & 0.4967061 & 1.2056220 & -3.2037078 \\ \mathrm{C} & 0.2142121 & -0.0652492 & -3.7990280 \\ \mathrm{Cl} & 1.4277130 & 2.7839643 & -1.2147169 \\ \mathrm{Cl} & 0.1423123 & 2.6514219 & -4.0951357 \\ \mathrm{Cl} & 0.1934384 & -2.7991462 & -3.8810526 \\ \mathrm{Cl} & 1.5049007 & -2.6725270 & -1.0103662 \\ \mathrm{Cl} & -0.4978840 & -0.1317245 & -5.3597539 \\ \mathrm{O} & 2.5534728 & 0.1412974 & -0.4996621 \\ \mathrm{H} & 2.6216630 & -0.5637666 & 0.1877227 \\ & & & \end{array}$

$\begin{array}{rrrrrr}0.00 & 0.00 & 0.00 & 0.00 & 0.00 & 0.00 \\ 9.20 & 16.45 & 27.62 & 40.95 & 42.99 & 58.44 \\ 62.28 & 68.44 & 70.07 & 81.47 & 97.34 & 103.26 \\ 112.07 & 136.24 & 148.79 & 149.70 & 164.42 & 180.52 \\ 189.71 & 204.01 & 207.75 & 208.83 & 213.11 & 220.43 \\ 234.19 & 253.61 & 261.02 & 269.52 & 273.57 & 276.02 \\ 284.75 & 291.69 & 293.58 & 299.53 & 303.71 & 315.76 \\ 324.55 & 345.59 & 353.75 & 358.55 & 359.02 & 363.12 \\ 366.60 & 380.80 & 395.59 & 400.53 & 407.70 & 419.82 \\ 420.22 & 421.49 & 422.15 & 459.34 & 527.52 & 564.96 \\ 577.90 & 637.23 & 651.22 & 656.45 & 657.89 & 658.50 \\ 673.01 & 673.66 & 683.02 & 694.18 & 696.16 & 698.35 \\ 709.54 & 717.75 & 723.27 & 734.34 & 736.01 & 761.78 \\ 768.92 & 770.57 & 771.40 & 778.04 & 784.43 & 784.63 \\ 785.53 & 799.25 & 837.02 & 840.04 & 844.67 & 846.83 \\ 853.36 & 886.68 & 891.30 & 892.32 & 894.49 & 915.30 \\ 991.24 & 1001.35 & 1003.11 & 1010.87 & 1013.59 & 1031.46 \\ 1032.05 & 1035.64 & 1038.62 & 1040.85 & 1042.33 & 1046.92 \\ 1054.32 & 1062.21 & 1076.88 & 1111.70 & 1140.05 & 1145.88 \\ 1148.55 & 1182.05 & 1183.31 & 1236.69 & 1260.74 & 1262.90 \\ 1301.10 & 1326.84 & 1328.65 & 1331.24 & 1334.23 & 1343.18 \\ 1364.53 & 1375.78 & 1383.33 & 1390.83 & 1395.72 & 1416.22 \\ 1468.01 & 1472.05 & 1472.11 & 1505.22 & 1509.62 & 1521.79 \\ 1555.61 & 1557.12 & 1578.51 & 1605.73 & 1609.90 & 1615.06 \\ 1658.57 & 2534.69 & 3122.73 & 3124.52 & 3126.45 & 3126.87 \\ 3154.46 & 3154.65 & 3154.93 & 3155.25 & 3173.43 & 3173.77 \\ 3173.95 & 3174.35 & 3484.49 & & & \end{array}$

TURBOMOLE Energies and Thermochemistry ${ }^{1}$ $\mathrm{RI}-\mathrm{PBE} / \mathrm{TZVP}, \mathrm{SV}(\mathrm{P})$

$-5326.823287$

ZP

RI-PBE/TZVP,SV $(P)+Z P E$

0.3352465

RI-PBE/TZVPP

B3-LYP/TZVP,SV(P)

$\mathrm{ZPE}(\mathrm{kJ} / \mathrm{mol})$

$-5326.488041$

$-5329.250231$

$-5328.493331$

880.2

$\ln (q$ trans)

20.39

In(qrot)

17.48

In(qvib)

34.03

chem.pot. $(\mathrm{kJ} / \mathrm{mol})$

701.97

energy $(\mathrm{kJ} / \mathrm{mol})$

972.21

entropy $(\mathrm{kJ} / \mathrm{mol} / \mathrm{K})$

0.91471

$\mathrm{Cv}(\mathrm{kJ} / \mathrm{mol}-\mathrm{K})$

0.5629781

$\mathrm{Cp}((\mathrm{kJ} / \mathrm{mol}-\mathrm{K})$

0.5712924

$\mathrm{RI}$ - PBE/TZVP, SV (P) $<\mathrm{S}^{2}>$

1.32

$\mathrm{RI}-\mathrm{PBE} / \mathrm{TZVPP}<\mathrm{S}^{2}>$

1.32

B3 - LYP / TZVP, SV (P) $\quad<\mathrm{S}^{2}>$

1.72

${ }^{1}$ Freeh output; $298.15 \mathrm{~K}, 0.1 \mathrm{MPa}$, unscaled

Vibrational frequencies 


\section{Oxo-Complex}

\begin{tabular}{lrrr} 
lu & \multicolumn{3}{l}{} \\
Energy $=-2678.183068813$ & \\
$\mathrm{C}$ & -1.2588748 & -0.8443384 & -0.0527939 \\
$\mathrm{C}$ & 0.0065598 & -1.6570941 & 0.2302542 \\
$\mathrm{C}$ & 1.2727461 & -0.8133721 & -0.0233145 \\
$\mathrm{C}$ & 1.2340901 & 0.5795554 & -0.0044069 \\
$\mathrm{C}$ & -0.0148752 & 1.2815971 & 0.0579259 \\
$\mathrm{C}$ & -1.2408719 & 0.5553806 & -0.0102998 \\
$\mathrm{Cl}$ & -0.0285335 & 2.9978471 & 0.1627920 \\
$\mathrm{Cl}$ & 2.6928196 & 1.4831274 & -0.1782089 \\
$\mathrm{Cl}$ & 2.7409319 & -1.6863191 & -0.1801626 \\
$\mathrm{Cl}$ & -2.7222346 & 1.4300835 & -0.1333382 \\
$\mathrm{Cl}$ & -2.7166298 & -1.7218196 & -0.2034350 \\
$\mathrm{O}$ & -0.0212189 & -2.8300069 & -0.5366958 \\
$\mathrm{H}$ & 0.5622385 & -3.4687456 & -0.0678391 \\
$\mathrm{O}$ & 0.0612748 & -1.8233135 & 1.5724410
\end{tabular}

Vibrational frequencies

$\begin{array}{rrrrrr}0.00 & 0.00 & 0.00 & 0.00 & 0.00 & 0.00 \\ 39.69 & 61.98 & 77.96 & 104.23 & 136.72 & 191.08 \\ 198.39 & 206.71 & 207.35 & 219.42 & 268.18 & 280.19 \\ 291.65 & 296.80 & 334.58 & 357.08 & 389.00 & 501.82 \\ 544.32 & 571.30 & 631.90 & 636.48 & 708.07 & 756.59 \\ 855.99 & 912.44 & 1001.63 & 1062.77 & 1082.56 & 1150.41 \\ 1225.67 & 1302.09 & 1355.59 & 1389.59 & 1446.62 & 3532.74\end{array}$

TURBOMOLE Energies and Thermochemistry ${ }^{1}$

\begin{tabular}{lr}
\hline RI-PBE/TZVP,SV(P) & -2678.251213 \\
\hline ZPE & 0.0579579 \\
RI-PBE/TZVP,SV(P) + ZPE & -2678.193255 \\
RI-PBE/TZVPP & -2679.479931 \\
B3-LYP/TZVP,SV(P) & -2679.117287 \\
ZPE (kJ/mol) & 152.2 \\
In(qtrans) & 19.07 \\
In(qrot) & 15.14 \\
In(qvib) & 8.61 \\
chem.pot. (kJ/mol) & 46.03 \\
energy $(\mathrm{kJ} / \mathrm{mol})$ & 185.27 \\
entropy $(\mathrm{kJ} / \mathrm{mol} / \mathrm{K})$ & 0.47534 \\
Cv $(\mathrm{kJ} / \mathrm{mol}-\mathrm{K})$ & 0.1869075 \\
Cp $((\mathrm{kJ} / \mathrm{mol}-\mathrm{K})$ & 0.1952218 \\
\hline
\end{tabular}

${ }^{1}$ Freeh output; 298.15K, 0.1MPa, unscaled 
${ }^{4} \mathbf{A}$

Pentachlorophenol: [(Porphyrin $)(\mathrm{Fe}=\mathbf{O})(\mathrm{SH})]$ Prereactant Complex (ipso-addition)

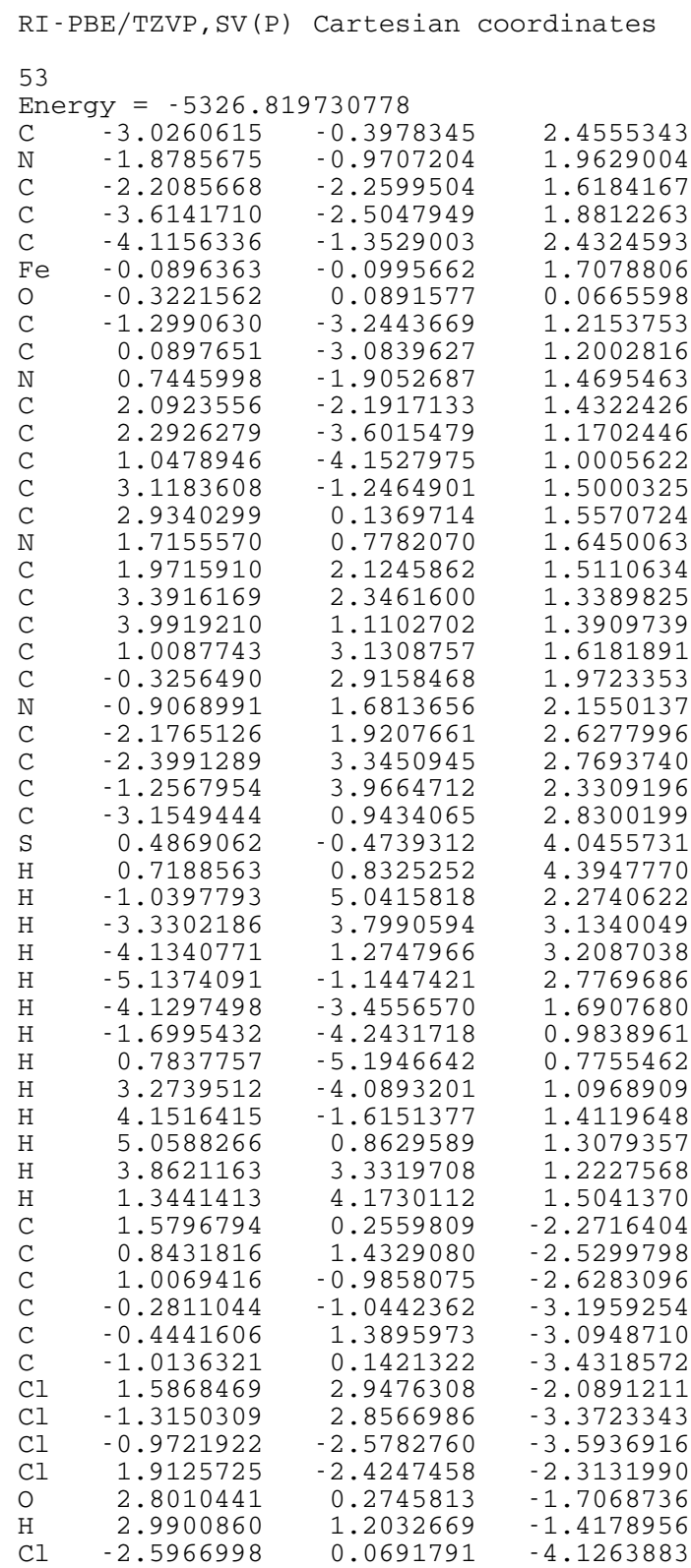

$\begin{array}{rrrrrr}0.00 & 0.00 & 0.00 & 0.00 & 0.00 & 0.00 \\ 8.31 & 10.71 & 18.48 & 25.10 & 28.15 & 53.01 \\ 56.24 & 58.59 & 77.80 & 81.10 & 84.35 & 90.47 \\ 103.54 & 118.50 & 123.76 & 143.67 & 150.37 & 171.17 \\ 173.88 & 179.70 & 199.82 & 204.14 & 208.22 & 212.48 \\ 213.73 & 220.46 & 234.63 & 267.28 & 271.53 & 272.95 \\ 279.87 & 286.71 & 298.23 & 313.68 & 315.88 & 319.20 \\ 329.64 & 332.16 & 336.59 & 345.06 & 358.61 & 360.54 \\ 370.11 & 372.24 & 403.15 & 406.77 & 419.79 & 421.02 \\ 422.30 & 424.16 & 452.33 & 458.07 & 469.44 & 541.36 \\ 555.18 & 564.41 & 631.62 & 635.33 & 650.74 & 652.50 \\ 654.11 & 667.00 & 673.12 & 691.80 & 692.07 & 694.27 \\ 698.17 & 713.84 & 724.26 & 734.03 & 735.02 & 758.95 \\ 762.68 & 769.27 & 769.73 & 775.03 & 778.71 & 785.59 \\ 786.55 & 799.04 & 801.24 & 842.62 & 850.70 & 852.45 \\ 860.32 & 866.92 & 885.73 & 890.39 & 891.97 & 893.91 \\ 977.77 & 994.64 & 1003.97 & 1005.03 & 1011.05 & 1016.88 \\ 1030.57 & 1033.67 & 1036.26 & 1040.70 & 1041.05 & 1043.12 \\ 1056.11 & 1112.55 & 1138.31 & 1146.70 & 1148.86 & 1181.53 \\ 1185.26 & 1216.82 & 1261.56 & 1263.05 & 1274.25 & 1295.26 \\ 1326.25 & 1326.76 & 1338.53 & 1352.15 & 1363.30 & 1373.11 \\ 1380.41 & 1380.93 & 1385.18 & 1394.87 & 1432.67 & 1463.21 \\ 1466.81 & 1468.15 & 1500.36 & 1519.76 & 1550.80 & 1553.20 \\ 1554.00 & 1571.87 & 1573.32 & 1580.11 & 1600.35 & 1605.53 \\ 1653.89 & 2549.81 & 3124.55 & 3125.60 & 3126.89 & 3126.97 \\ 3153.43 & 3155.53 & 3155.66 & 3155.85 & 3172.13 & 3174.30 \\ 3174.45 & 3174.63 & 3409.66 & & & \end{array}$

TURBOMOLE Energies and Thermochemistry ${ }^{1}$ $\mathrm{RI}-\mathrm{PBE} / \mathrm{TZVP}, \mathrm{SV}(\mathrm{P})$ $-5326.819731$

ZPE

RI-PBE/TZVP,SV $(P)+Z P E$

0.3351145

RI-PBE/TZVPP

B3-LYP/TZVP,SV(P)

$\mathrm{ZPE}(\mathrm{kJ} / \mathrm{mol})$

$\ln$ (qtrans)

$-5326.484616$

$-5329.252564$

$-5328.493633$

879.8

20.39

In(qrot)

17.51

In(qvib)

37.08

chem.pot. $(\mathrm{kJ} / \mathrm{mol})$

693.97

energy $(\mathrm{kJ} / \mathrm{mol})$

973.62

entropy $(\mathrm{kJ} / \mathrm{mol} / \mathrm{K})$

0.94627

$\mathrm{Cv}(\mathrm{kJ} / \mathrm{mol}-\mathrm{K})$

0.5657577

$\mathrm{Cp}((\mathrm{kJ} / \mathrm{mol}-\mathrm{K})$

0.574072

3.77

RI - PBE/TZVP, SV (P) $\quad\left\langle\mathrm{S}^{2}>\right.$

3.77

$\mathrm{RI}-\mathrm{PBE} / \mathrm{TZVPP}<\mathrm{S}^{2}>$

3.80

${ }^{1}$ Freeh output; $298.15 \mathrm{~K}, 0.1 \mathrm{MPa}$, unscaled 
${ }^{4} \mathrm{~A}$

\section{Pentachlorophenol:[(Porphyrin $)(\mathrm{Fe}=\mathbf{O})(\mathrm{SH})]$} Transition State (ipso-addition)

\begin{tabular}{|c|c|c|c|}
\hline \multicolumn{2}{|c|}{$\mathrm{RI}-\mathrm{PBE} / \mathrm{TZVP}, \mathrm{SV}(\mathrm{P})$} & Cartesian & coordinates \\
\hline & & & \\
\hline & $y=-5326.80$ & 575513 & \\
\hline C & -3.3109688 & -0.6539373 & 0.5097052 \\
\hline & -2.0910950 & -1.1254303 & 0.9313907 \\
\hline & -2.1444677 & -2.4933105 & 0.7939499 \\
\hline & -3.4314018 & -2.8984602 & 0.2626653 \\
\hline & -4.1625113 & -1.7515945 & 0.0935049 \\
\hline $\mathrm{Fe}$ & -0.5102863 & -0.0263887 & 1.5407040 \\
\hline $\mathrm{O}$ & 0.0568125 & 0.0034128 & -0.1070490 \\
\hline C & -1.1318547 & -3.3816578 & 1.1592841 \\
\hline C & 0.0855400 & -3.0148317 & 1.7397765 \\
\hline $\mathrm{N}$ & 0.4901713 & -1.7262329 & 1.9918211 \\
\hline C & 1.7383301 & -1.8238277 & 2.5664955 \\
\hline C & 2.1272892 & -3.2131925 & 2.6903459 \\
\hline C & 1.0987674 & -3.9562663 & 2.1698497 \\
\hline $\mathrm{C}$ & 2.5431576 & -0.7497079 & 2.9498799 \\
\hline $\mathrm{C}$ & 2.2099234 & 0.5998623 & 2.8097022 \\
\hline $\mathrm{N}$ & 1.0366108 & 1.0814258 & 2.2660488 \\
\hline C & 1.1265772 & 2.4539075 & 2.3196379 \\
\hline C & 2.3935874 & 2.8546393 & 2.8934682 \\
\hline C & 3.0678269 & 1.6985943 & 3.2022838 \\
\hline C & 0.1322924 & 3.3413332 & 1.8995741 \\
\hline C & -1.1247306 & 2.9660751 & 1.4199126 \\
\hline $\mathrm{N}$ & -1.5679037 & 1.6701377 & 1.2636392 \\
\hline C & -2.8620845 & 1.7664475 & 0.8014643 \\
\hline C & -3.2514128 & 3.1556039 & 0.6741848 \\
\hline C & -2.1654898 & 3.9036097 & 1.0514980 \\
\hline C & -3.6857496 & 0.6903738 & 0.4642321 \\
\hline S & -1.2218336 & -0.1479300 & 3.7646611 \\
\hline $\mathrm{H}$ & -1.426 & 6296 & 3.96 \\
\hline $\mathrm{H}$ & -2.0624030 & 4.9963664 & 1.0874393 \\
\hline $\mathrm{H}$ & -4.2358781 & 3.4987105 & 0.3291229 \\
\hline $\mathrm{H}$ & -4.7007374 & 0.9224487 & 0.1064074 \\
\hline $\mathrm{H}$ & -5.1883413 & -1.6388604 & -0.2820403 \\
\hline $\mathrm{H}$ & -3.7242174 & -3.9372698 & 0.0597801 \\
\hline $\mathrm{H}$ & -1.3173056 & -4.4556295 & 1.0045737 \\
\hline $\mathrm{H}$ & 1.0159959 & -5.0475362 & 2.0789541 \\
\hline $\mathrm{H}$ & 3.0767802 & -3.5593786 & 3.1199622 \\
\hline $\mathrm{H}$ & 3.5234072 & -0.9853115 & 3.3920283 \\
\hline $\mathrm{H}$ & 4.0576855 & 1.5839590 & 3.6641158 \\
\hline $\mathrm{H}$ & 2.7103045 & 3.8952094 & 3.04 \\
\hline $\mathrm{H}$ & 0.342 & 4.4185534 & 1.98373 \\
\hline C & 1.6289188 & -0.06631 & -1.1507406 \\
\hline C & 1.4233929 & 1.2148838 & -1.8122033 \\
\hline C & 1.2946275 & -1.2435728 & -1.9342093 \\
\hline C & 0.5259333 & -1.1324093 & -3.0964382 \\
\hline C & 0.6474354 & 1.3233571 & -2.9659312 \\
\hline $\mathrm{C}$ & 0.1845747 & 0.1469792 & -3.6169443 \\
\hline $\mathrm{Cl}$ & 2.0907601 & 2.6075941 & -1.0346631 \\
\hline $\mathrm{Cl}$ & 0.2958807 & 2.8786747 & -3.6364666 \\
\hline $\mathrm{Cl}$ & 0.0210852 & -2.5575182 & -3.9362267 \\
\hline $\mathrm{Cl}$ & 1.7920765 & -2.7730789 & -1.3290725 \\
\hline $\mathrm{O}$ & 2.6291236 & -0.2138043 & -0.2619924 \\
\hline $\mathrm{H}$ & 2.7313092 & 0.6404724 & 0.2268414 \\
\hline $\mathrm{Cl}$ & -0.7837091 & 0.2732657 & -5.0360578 \\
\hline
\end{tabular}

$\begin{array}{rrrrrr}1460.03 & 0.00 & 0.00 & 0.00 & 0.00 & 0.00 \\ 0.00 & 3.45 & 24.38 & 29.93 & 37.70 & 47.25 \\ 52.99 & 54.45 & 68.33 & 77.81 & 84.39 & 97.07 \\ 100.12 & 105.90 & 140.17 & 149.67 & 153.64 & 163.57 \\ 199.73 & 200.81 & 205.11 & 206.65 & 207.99 & 215.15 \\ 216.27 & 232.23 & 248.57 & 257.40 & 272.28 & 276.64 \\ 286.36 & 288.46 & 290.36 & 299.37 & 314.11 & 319.16 \\ 324.61 & 333.36 & 345.03 & 350.72 & 357.98 & 358.93 \\ 365.03 & 375.10 & 402.39 & 406.44 & 416.84 & 418.74 \\ 419.65 & 421.03 & 436.42 & 456.75 & 458.84 & 474.76 \\ 556.54 & 602.02 & 609.14 & 636.56 & 652.36 & 656.30 \\ 657.56 & 672.45 & 675.67 & 686.11 & 694.86 & 695.32 \\ 698.19 & 701.37 & 717.16 & 724.14 & 734.81 & 735.87 \\ 763.18 & 763.67 & 771.42 & 772.11 & 778.07 & 781.92 \\ 784.45 & 784.82 & 798.73 & 839.94 & 848.64 & 849.71 \\ 857.51 & 863.59 & 886.76 & 891.58 & 892.41 & 894.19 \\ 962.35 & 992.30 & 1000.80 & 1002.64 & 1009.87 & 1014.34 \\ 1030.92 & 1031.67 & 1035.45 & 1040.57 & 1040.60 & 1041.98 \\ 1054.10 & 1095.97 & 1140.97 & 1147.95 & 1149.94 & 1181.76 \\ 1183.46 & 1184.16 & 1251.76 & 1261.40 & 1263.75 & 1314.16 \\ 1321.41 & 1329.15 & 1329.43 & 1340.04 & 1361.38 & 1363.88 \\ 1376.61 & 1386.36 & 1389.74 & 1394.55 & 1398.49 & 1460.11 \\ 1467.32 & 1470.35 & 1471.65 & 1502.30 & 1519.61 & 1523.66 \\ 1555.85 & 1556.95 & 1577.72 & 1597.81 & 1607.23 & 1611.12 \\ 1660.48 & 2547.36 & 3122.09 & 3122.17 & 3123.11 & 3123.96 \\ 3153.74 & 3154.66 & 3154.83 & 3155.08 & 3172.61 & 3173.79 \\ 3173.94 & 3174.18 & 3444.71 & & & \end{array}$

TURBOMOLE Energies and Thermochemistry ${ }^{1}$ $\mathrm{RI}-\mathrm{PBE} / \mathrm{TZVP}, \mathrm{SV}(\mathrm{P})$ $-5326.800576$

ZPE

RI-PBE/TZVP,SV(P) + ZPE

0.3343929

RI-PBE/TZVPP

B3-LYP/TZVP,SV(P)

$\mathrm{ZPE}(\mathrm{kJ} / \mathrm{mol})$

$-5326.466183$

$-5329.230411$

$-5328.46813$

877.9

$\ln$ (qtrans)

20.39

$\ln ($ qrot)

17.46

In(qvib)

34.41

chem.pot. $(\mathrm{kJ} / \mathrm{mol})$

698.82

energy $(\mathrm{kJ} / \mathrm{mol})$

969.31

entropy $(\mathrm{kJ} / \mathrm{mol} / \mathrm{K})$

0.91554

Cv (kJ/mol-K)

0.5589194

$\mathrm{Cp}((\mathrm{kJ} / \mathrm{mol}-\mathrm{K})$

0.5672337

RI - PBE/TZVP, SV (P) $\quad\left\langle\mathrm{S}^{2}>\right.$

3.77

$\mathrm{RI}-\mathrm{PBE} / \mathrm{TZVPP}<\mathrm{S}^{2}>$

3.76

B3 - LYP/TZVP, SV (P) $<\mathrm{S}^{2}>$

3.81

${ }^{1}$ Freeh output; $298.15 \mathrm{~K}, 0.1 \mathrm{MPa}$, unscaled 
${ }^{4} \mathbf{A}$

\section{Pentachlorophenol: $[($ Porphyrin $)(\mathrm{Fe}=\mathbf{O})(\mathrm{SH})]$ $\sigma$-Complex (ipso-addition)}

RI-PBE/TZVP, SV(P) Cartesian coordinates

$$
53
$$$$
\text { Energy }=-5326.821205874
$$

\begin{tabular}{|c|c|c|c|}
\hline C & -3.2698922 & -0.2839828 & 0.5224987 \\
\hline $\mathrm{N}$ & -2.1338127 & -0.9147667 & 0.9682426 \\
\hline C & -2.3688163 & -2.2635556 & 0.8451404 \\
\hline C & -3.6900069 & -2.4948895 & 0.2950397 \\
\hline C & -4.2498453 & -1.2607868 & 0.0887341 \\
\hline $\mathrm{Fe}$ & -0.4333068 & -0.0293724 & 1.6393792 \\
\hline 0 & 0.0800751 & 0.0059492 & -0.1592632 \\
\hline C & -1.4790334 & -3.2749212 & 1.2062470 \\
\hline C & -0.2039073 & -3.0640624 & 1.7344432 \\
\hline $\mathrm{N}$ & 0.3806470 & -1.8374877 & 1.9704876 \\
\hline C & 1.6478686 & -2.1007975 & 2.4467291 \\
\hline C & 1.8672618 & -3.5278260 & 2.516409 \\
\hline C & 0.7123956 & -4.1275743 & 2.078925 \\
\hline C & 2.5977788 & -1.1481259 & 2.8176913 \\
\hline $\mathrm{C}$ & 2.4043277 & 0.2326779 & 2.7741308 \\
\hline $\mathrm{N}$ & 1.2665731 & 0.8675299 & 2.3122484 \\
\hline C & 1.4656164 & 2.2156490 & 2.5346163 \\
\hline C & 2.7769639 & 2.4408931 & 3.1010309 \\
\hline C & 3.3635186 & 1.2072319 & 3.2443869 \\
\hline C & 0.5338033 & 3. 2222317 & 2.2798147 \\
\hline C & -0.7499730 & 3.0091496 & 1.773515 \\
\hline $\mathrm{N}$ & -1.3033486 & 1.7825257 & 1.467225 \\
\hline C & -2.5599931 & 2.0463417 & 0.9612 \\
\hline C & -2.8084165 & 3.4709098 & 0.95751 \\
\hline C & -1.6848484 & 4.0697795 & 1.470911 \\
\hline C & -3.4784156 & 1.0958486 & 0.511813 \\
\hline S & -1.1707413 & -0.2105187 & 3.7710202 \\
\hline $\mathrm{H}$ & -1.5953340 & 1.0837774 & 3.9391949 \\
\hline $\mathrm{H}$ & -1.4852273 & 5.1389761 & 1.6229419 \\
\hline $\mathrm{H}$ & -3.7348714 & 3.9402250 & 0.6006380 \\
\hline $\mathrm{H}$ & -4.4426941 & 1.4636768 & 0.1300981 \\
\hline $\mathrm{H}$ & -5.2415044 & -1.0135331 & -0.3131974 \\
\hline $\mathrm{H}$ & -4.1174473 & -3.4866121 & 0.096055 \\
\hline $\mathrm{H}$ & -1.8028301 & -4.3166537 & 1.061980 \\
\hline $\mathrm{H}$ & 0.4887229 & -5.1983623 & 1.981379 \\
\hline $\mathrm{H}$ & 2.7977527 & -3.9980753 & 2.86126 \\
\hline $\mathrm{H}$ & 3.5654488 & -1.5147631 & 3.19148 \\
\hline $\mathrm{H}$ & 4.3539748 & 0.9610605 & $3.64966^{\circ}$ \\
\hline $\mathrm{H}$ & 3.1832592 & 3.4280026 & 3.3589456 \\
\hline $\mathrm{H}$ & 0.8318192 & 4.2602304 & 2.4920449 \\
\hline $\mathrm{C}$ & 1.1842703 & 0.0016700 & -1.0162159 \\
\hline $\mathrm{C}$ & 1.0677786 & 1.2815367 & -1.8539801 \\
\hline C & 1.0616192 & -1.2238474 & -1.9197062 \\
\hline C & 0.5128122 & -1.1650921 & -3.191753 \\
\hline C & 0.5313141 & 1.3135549 & -3.128181 \\
\hline $\mathrm{C}$ & 0.2173748 & 0.0923764 & -3.805918 \\
\hline $\mathrm{Cl}$ & 03807 & 2.7295178 & -1.042464 \\
\hline $\mathrm{Cl}$ & 0.27 & 2.8248134 & -3.94 \\
\hline $\mathrm{Cl}$ & 0.2149951 & -2.6242943 & -4.08351 \\
\hline $\mathrm{Cl}$ & 1.50 & -2.7221248 & -1.2 \\
\hline $\mathrm{O}$ & 2.4 & -0.0730484 & -0.3 \\
\hline $\mathrm{H}$ & 2.3 & 0.6311615 & 0.33 \\
\hline $\mathrm{Cl}$ & -0.5 & 0.1400243 & -5.36264 \\
\hline
\end{tabular}

$\begin{array}{rrrrrr}0.00 & 0.00 & 0.00 & 0.00 & 0.00 & 0.00 \\ 12.81 & 20.83 & 33.86 & 42.62 & 53.48 & 58.03 \\ 60.71 & 69.84 & 76.76 & 82.10 & 95.16 & 106.37 \\ 110.70 & 134.29 & 145.41 & 150.14 & 155.20 & 189.80 \\ 204.07 & 205.63 & 207.27 & 210.88 & 213.05 & 219.02 \\ 227.96 & 245.32 & 255.13 & 267.85 & 273.23 & 275.32 \\ 275.90 & 279.00 & 287.48 & 294.55 & 308.62 & 312.74 \\ 316.11 & 342.19 & 355.17 & 355.80 & 357.76 & 365.71 \\ 373.95 & 392.12 & 400.07 & 400.51 & 417.89 & 419.74 \\ 420.80 & 423.05 & 427.72 & 458.19 & 533.54 & 562.66 \\ 581.80 & 636.03 & 637.97 & 648.24 & 655.88 & 658.11 \\ 669.15 & 674.18 & 684.72 & 694.36 & 695.10 & 696.01 \\ 709.70 & 719.05 & 723.58 & 733.40 & 735.62 & 761.60 \\ 765.05 & 771.34 & 772.03 & 779.24 & 782.82 & 783.59 \\ 787.01 & 798.24 & 834.79 & 839.40 & 843.33 & 845.10 \\ 851.86 & 888.70 & 893.41 & 893.67 & 895.34 & 918.10 \\ 958.18 & 992.98 & 1000.39 & 1003.21 & 1012.89 & 1016.45 \\ 1032.39 & 1032.57 & 1036.43 & 1041.90 & 1042.02 & 1043.17 \\ 1054.70 & 1065.42 & 1088.26 & 1121.68 & 1140.82 & 1145.96 \\ 1147.82 & 1182.11 & 1183.36 & 1235.74 & 1260.85 & 1261.30 \\ 1305.43 & 1329.53 & 1332.56 & 1333.10 & 1336.04 & 1346.69 \\ 1365.25 & 1377.44 & 1382.89 & 1385.28 & 1394.67 & 1424.34 \\ 1470.02 & 1470.82 & 1474.81 & 1506.47 & 1508.33 & 1521.59 \\ 1554.23 & 1557.16 & 1579.04 & 1603.74 & 1608.77 & 1622.37 \\ 1661.53 & 2544.43 & 3124.89 & 3127.22 & 3127.52 & 3128.66 \\ 3155.26 & 3155.69 & 3155.90 & 3156.12 & 3174.09 & 3174.61 \\ 3174.76 & 3174.87 & 3502.68 & & & \end{array}$

TURBOMOLE Energies and Thermochemistry ${ }^{1}$ $\mathrm{RI}-\mathrm{PBE} / \mathrm{TZVP}, \mathrm{SV}(\mathrm{P})$ $-5326.823287$

ZPE

RI-PBE/TZVP,SV(P) + ZPE

0.3352465

RI-PBE/TZVPP

B3-LYP/TZVP,SV(P)

$\mathrm{ZPE}(\mathrm{kJ} / \mathrm{mol})$

5326.488041

$-5329.250231$

$-5328.493331$

880.2

$\ln$ (qtrans)

20.39

In(qrot)

17.48

In(qvib)

34.03

chem.pot. $(\mathrm{kJ} / \mathrm{mol})$

701.97

energy $(\mathrm{kJ} / \mathrm{mol})$

972.21

entropy $(\mathrm{kJ} / \mathrm{mol} / \mathrm{K})$

0.91471

Cv (kJ/mol-K)

0.5629781

$\mathrm{Cp}((\mathrm{kJ} / \mathrm{mol}-\mathrm{K})$

0.5712924

RI - PBE/TZVP, SV (P) $\quad<\mathrm{S}^{2}>$

$\mathrm{RI}-\mathrm{PBE} / \mathrm{TZVPP}<\mathrm{S}^{2}>$

3.79

B3 - LYP/TZVP, SV (P) $<\mathrm{S}^{2}>$

3.80

${ }^{1}$ Freeh output; $298.15 \mathrm{~K}, 0.1 \mathrm{MPa}$, unscaled 


\section{Chloropentafluorobenzene (C2v)}

\begin{tabular}{lrrr} 
l2 & \multicolumn{3}{l}{} \\
Energy $=-1186.388943632$ & \\
C & 1.2172581 & 0.0000000 & -0.4139613 \\
C & 1.2175681 & 0.0000000 & 0.9914507 \\
C & 0.0000000 & 0.0000000 & 1.6938555 \\
C & -1.2175681 & 0.0000000 & 0.9914507 \\
C & -1.2172581 & 0.0000000 & -0.4139613 \\
C & 0.0000000 & 0.0000000 & -1.1220401 \\
C1 & 0.0000000 & 0.0000000 & -2.8466254 \\
F & 0.0000000 & 0.0000000 & 3.0263368 \\
F & -2.3698317 & 0.0000000 & 1.6637865 \\
F & -2.3782425 & 0.0000000 & -1.0667607 \\
F & 2.3698317 & 0.0000000 & 1.6637865 \\
F & 2.3782425 & 0.0000000 & -1.0667607
\end{tabular}

Vibrational frequencies

$\begin{array}{rrrrrr}0.00 & 0.00 & 0.00 & 0.00 & 0.00 & 0.00 \\ 105.22 & 129.38 & 145.88 & 192.59 & 206.02 & 259.57 \\ 260.74 & 297.77 & 299.54 & 346.71 & 377.22 & 385.69 \\ 412.34 & 429.06 & 507.41 & 569.81 & 605.94 & 617.11 \\ 752.52 & 874.73 & 999.95 & 1108.66 & 1177.84 & 1299.81\end{array}$

TURBOMOLE Energies and Thermochemistry ${ }^{1}$

RI-PBE/TZVP,SV(P)

ZPE

RI-PBE/TZVP,SV(P) + ZPE

RI-PBE/TZVPP

B3-LYP/TZVP,SV(P)

$\mathrm{ZPE}(\mathrm{kJ} / \mathrm{mol})$

$\ln$ (qtrans)

$\ln$ (qrot)

In(qvib)

chem.pot. $(\mathrm{kJ} / \mathrm{mol})$

energy $(\mathrm{kJ} / \mathrm{mol})$

entropy $(\mathrm{kJ} / \mathrm{mol} / \mathrm{K})$

Cv $(\mathrm{kJ} / \mathrm{mol}-\mathrm{K})$

$\mathrm{Cp}((\mathrm{kJ} / \mathrm{mol}-\mathrm{K})$
$-1186.388944$

0.0488929

$-1186.340051$

$-1187.402455$

$-1186.930176$

128.4

18.57

13.57

5.71

34.55

155.61

0.41435

0.153943

0.1622573

${ }^{1}$ Freeh output; 298.15K, 0.1MPa, unscaled 
${ }^{2} \mathbf{A}$

\section{Chloropentafluorobenzene: $[($ Porphyrin $)(\mathrm{Fe}=$ O)(SH)] Prereactant Complex (Cl-addition)}

RI-PBE/TZVP, SV (P) Cartesian coordinates

$$
52
$$$$
\text { Energy }=-3910.000130619
$$

\begin{tabular}{|c|c|c|c|}
\hline C & -1.1040641 & 2.7984354 & 0.1356583 \\
\hline $\mathrm{N}$ & -1.3664299 & 1.4461014 & 0.0808445 \\
\hline C & -2.2205562 & 1.2645582 & -0.9792750 \\
\hline $\mathrm{C}$ & -2.5228178 & 2.5334924 & -1.6086008 \\
\hline $\mathrm{C}$ & -1.8219587 & 3.4877803 & -0.9170913 \\
\hline $\mathrm{Fe}$ & -0.5237404 & -0.0058199 & 1.2357846 \\
\hline $\mathrm{O}$ & 0.8471058 & 0.0391755 & 0.3384604 \\
\hline C & -2.6965031 & 0.0265106 & -1.4191984 \\
\hline C & -2.3451643 & -1.2060899 & -0.8674790 \\
\hline $\mathrm{N}$ & -1.5055818 & -1.3986663 & 0.2114533 \\
\hline C & -1.4523900 & -2.7629295 & 0.4106461 \\
\hline $\mathrm{C}$ & -2.2630293 & -3.4472947 & -0.5734751 \\
\hline $\mathrm{C}$ & -2.8204219 & -2.4783220 & -1.3672081 \\
\hline $\mathrm{C}$ & -0.7469538 & -3.4058200 & 1.4278549 \\
\hline $\mathrm{C}$ & 0.0067232 & -2.7587189 & 2.4097338 \\
\hline $\mathrm{N}$ & 0.1790421 & -1.3971413 & 2.5073790 \\
\hline $\mathrm{C}$ & 1.0034011 & -1.1950991 & 3.5950993 \\
\hline $\mathrm{C}$ & 1.3413146 & -2.4625056 & 4.2092158 \\
\hline $\mathrm{C}$ & 0.7228484 & -3.4374097 & 3.4686170 \\
\hline C & 1.4787106 & 0.0375610 & 4.0514248 \\
\hline C & 1.2027100 & 1.2752958 & 3.4675658 \\
\hline $\mathrm{N}$ & 0.3892104 & 1.4636995 & 2.37 \\
\hline $\mathrm{C}$ & 0.4134819 & 2.8123744 & 2.10 \\
\hline $\mathrm{C}$ & 1.2527562 & 3.5004920 & 3.062 \\
\hline C & 1.7397938 & 2.5443251 & 3.9170380 \\
\hline $\mathrm{C}$ & -0.2762477 & 3.4378113 & 1.0614834 \\
\hline S & -2.3090589 & 0.5178386 & 2.6593079 \\
\hline $\mathrm{H}$ & -1.7849346 & 0.0293483 & 3.8305389 \\
\hline $\mathrm{H}$ & 2.4110903 & 2.6705123 & 4.7770862 \\
\hline $\mathrm{H}$ & 1.4343381 & 4.5835451 & 3.0695277 \\
\hline $\mathrm{H}$ & -0.1631947 & 4.5284833 & 0.9649566 \\
\hline $\mathrm{H}$ & -1.7805747 & 4.5709166 & -1.0926953 \\
\hline $\mathrm{H}$ & -3.1770374 & 2.6577864 & -2.4811358 \\
\hline $\mathrm{H}$ & -3.3692754 & 0.0192029 & -2.2897596 \\
\hline $\mathrm{H}$ & -3.4946219 & -2.5961515 & -2.2255742 \\
\hline $\mathrm{H}$ & -2.3836076 & -4.5371270 & -0.6313669 \\
\hline $\mathrm{H}$ & -0.7961251 & -4.5046533 & 1.4649624 \\
\hline $\mathrm{H}$ & 0.7418953 & -4.5259782 & 3.6115821 \\
\hline $\mathrm{H}$ & 1.9830176 & -2.5743966 & 5.0934246 \\
\hline $\mathrm{H}$ & 2.1404635 & 0.0272862 & 4.9312828 \\
\hline $\mathrm{C}$ & 2.2336444 & -0.5339537 & -2.2738973 \\
\hline $\mathrm{C}$ & 2.0356033 & 0.8557859 & -2.3684079 \\
\hline $\mathrm{C}$ & 1.2609981 & -1.4092956 & -2.7901248 \\
\hline $\mathrm{C}$ & 0.1131316 & -0.8996962 & -3.4175788 \\
\hline $\mathrm{C}$ & 0.8873308 & 1.3664791 & -2.9941659 \\
\hline $\mathrm{C}$ & -0.0701477 & 0.4878943 & -3.5251062 \\
\hline $\mathrm{Cl}$ & 3.6347332 & -1.1590993 & -1.4909712 \\
\hline $\mathrm{F}$ & 2.9274881 & 1.7029114 & -1.8609715 \\
\hline $\mathrm{F}$ & 0.6953834 & 2.6867041 & -3.0716248 \\
\hline $\mathrm{F}$ & -1.1690780 & 0.9705457 & -4.1159628 \\
\hline $\mathrm{F}$ & 44131 & -1.7322063 & -3.9035984 \\
\hline $\mathrm{F}$ & 1424 & -2.7297312 & -2.6860100 \\
\hline
\end{tabular}

$\begin{array}{rrrrrr}i 8.45 & 0.00 & 0.00 & 0.00 & 0.00 & 0.00 \\ 0.00 & 10.48 & 14.50 & 24.48 & 28.70 & 37.35 \\ 58.90 & 69.82 & 95.02 & 100.42 & 104.15 & 112.32 \\ 131.51 & 137.90 & 146.57 & 153.18 & 164.32 & 178.40 \\ 192.30 & 206.25 & 212.88 & 213.84 & 232.06 & 238.07 \\ 261.13 & 261.57 & 271.47 & 275.27 & 276.88 & 279.45 \\ 287.74 & 298.38 & 300.18 & 300.56 & 311.48 & 345.56 \\ 351.62 & 358.76 & 359.88 & 364.98 & 376.86 & 385.78 \\ 397.69 & 402.81 & 417.80 & 419.09 & 420.34 & 422.34 \\ 429.18 & 450.32 & 459.09 & 508.10 & 570.45 & 604.11 \\ 607.31 & 619.42 & 649.48 & 655.19 & 656.48 & 671.74 \\ 677.95 & 693.28 & 694.14 & 697.09 & 715.49 & 723.95 \\ 734.20 & 735.13 & 751.84 & 762.62 & 770.89 & 771.75 \\ 777.91 & 781.93 & 784.76 & 784.88 & 798.72 & 832.88 \\ 846.22 & 850.15 & 850.72 & 857.84 & 873.97 & 887.24 \\ 891.81 & 893.08 & 894.41 & 993.49 & 996.63 & 1002.36 \\ 1002.83 & 1011.38 & 1015.29 & 1032.50 & 1032.81 & 1036.98 \\ 1041.61 & 1041.81 & 1043.97 & 1054.48 & 1102.44 & 1139.53 \\ 1147.62 & 1148.75 & 1174.58 & 1182.91 & 1183.79 & 1261.47 \\ 1263.13 & 1291.06 & 1313.88 & 1329.04 & 1330.73 & 1341.37 \\ 1354.97 & 1363.54 & 1376.44 & 1382.65 & 1391.50 & 1392.38 \\ 1447.35 & 1469.34 & 1471.87 & 1472.54 & 1504.16 & 1514.22 \\ 1522.90 & 1525.97 & 1557.73 & 1558.30 & 1579.13 & 1602.16 \\ 1608.06 & 1609.73 & 1634.80 & 1635.18 & 1661.24 & 2543.87 \\ 3121.89 & 3123.92 & 3126.57 & 3130.37 & 3154.80 & 3155.50 \\ 3158.47 & 3159.16 & 3173.71 & 3174.49 & 3177.38 & 3178.53\end{array}$

TURBOMOLE Energies and Thermochemistry ${ }^{1}$

\begin{tabular}{|c|c|}
\hline RI-PBE/TZVP,SV(P) & -3910.000131 \\
\hline ZPE & 0.3297831 \\
\hline RI-PBE/TZVP,SV(P) + ZPE & -3909.670348 \\
\hline RI-PBE/TZVPP & -3912.306082 \\
\hline B3-LYP/TZVP,SV(P) & -3911.361661 \\
\hline ZPE (kJ/mol) & 865.8 \\
\hline In(qtrans) & 20.24 \\
\hline In(qrot) & 17.2 \\
\hline In(qvib) & 31.83 \\
\hline chem.pot. (kJ/mol) & 694.12 \\
\hline energy (kJ/mol) & 952.89 \\
\hline entropy $(\mathrm{kJ} / \mathrm{mol} / \mathrm{K})$ & 0.87625 \\
\hline $\mathrm{Cv}(\mathrm{kJ} / \mathrm{mol}-\mathrm{K})$ & 0.5374848 \\
\hline $\mathrm{Cp}((\mathrm{kJ} / \mathrm{mol}-\mathrm{K})$ & 0.5457991 \\
\hline $\mathrm{RI}-\mathrm{PBE} / \mathrm{TZVP}, \mathrm{SV}(\mathrm{P})<\mathrm{S}^{2}>$ & 1.12 \\
\hline $\mathrm{RI}-\mathrm{PBE} / \mathrm{TZVPP}<\mathrm{S}^{2}>$ & 1.10 \\
\hline B3 - LYP / TZVP , SV (P) $\quad\left\langle\mathrm{S}^{2}>\right.$ & 1.51 \\
\hline
\end{tabular}

${ }^{1}$ Freeh output; 298.15K, 0.1MPa， unscaled 
${ }^{2} \mathbf{A}$

\section{Chloropentafluorobenzene: $[($ Porphyrin $)(\mathrm{Fe}=$ O)(SH)] Transition State (Cl-addition)}

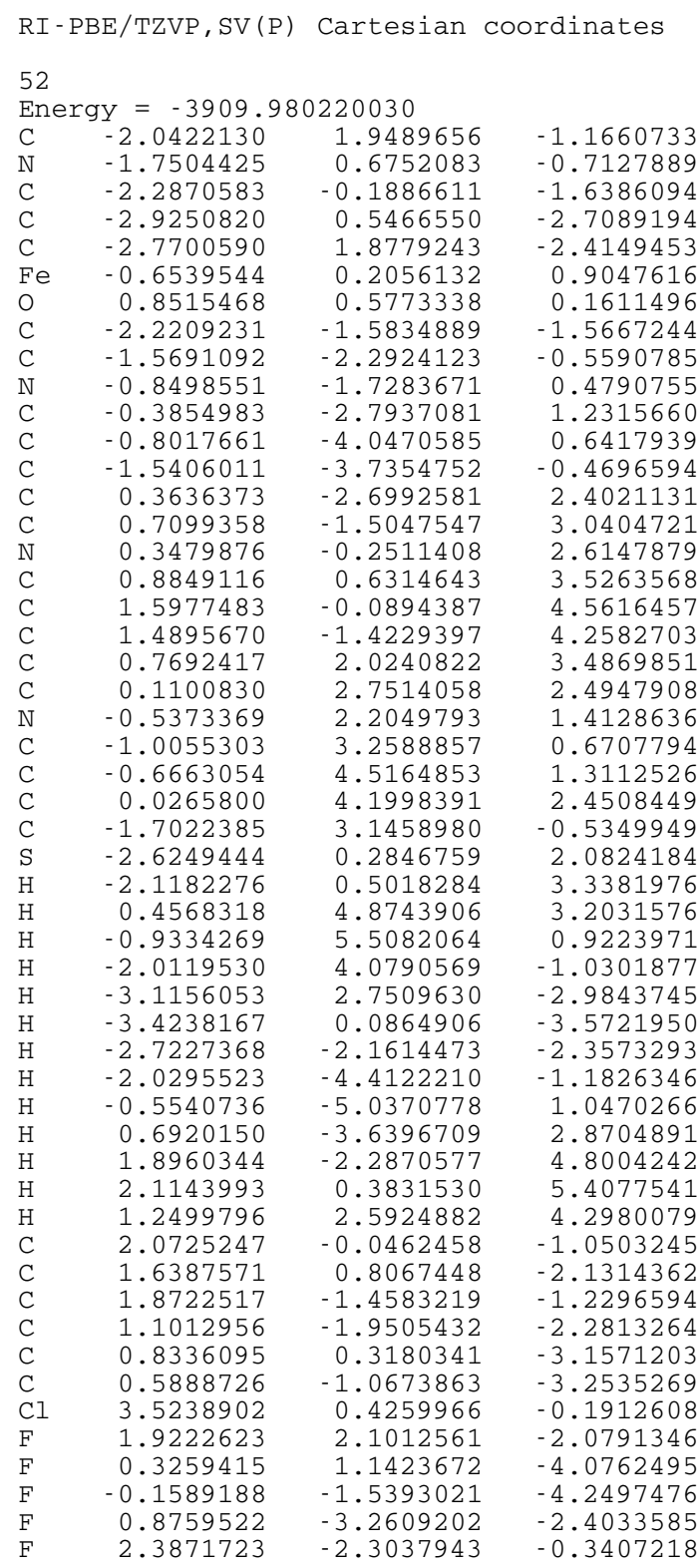

$\begin{array}{rrrrrr}i 480.71 & 0.00 & 0.00 & 0.00 & 0.00 & 0.00 \\ 0.00 & 7.10 & 14.79 & 43.31 & 47.87 & 49.04 \\ 57.65 & 69.01 & 86.13 & 96.03 & 105.15 & 120.89 \\ 124.58 & 137.16 & 144.35 & 149.59 & 154.19 & 172.60 \\ 187.88 & 201.82 & 208.42 & 220.96 & 232.44 & 256.47 \\ 259.90 & 261.19 & 268.93 & 270.37 & 272.31 & 280.25 \\ 288.61 & 295.15 & 299.20 & 302.24 & 320.14 & 346.30 \\ 350.47 & 358.00 & 361.49 & 375.59 & 380.37 & 382.37 \\ 396.45 & 408.00 & 417.54 & 419.58 & 421.29 & 421.58 \\ 422.17 & 458.44 & 493.90 & 537.44 & 565.83 & 608.38 \\ 612.13 & 635.95 & 651.26 & 655.25 & 657.93 & 672.66 \\ 679.61 & 688.93 & 694.42 & 696.85 & 698.11 & 716.86 \\ 723.49 & 734.31 & 735.92 & 749.69 & 763.48 & 770.83 \\ 771.83 & 778.12 & 782.90 & 785.01 & 785.19 & 798.73 \\ 834.58 & 844.71 & 847.36 & 849.84 & 857.54 & 886.02 \\ 890.50 & 892.54 & 894.47 & 991.17 & 993.59 & 999.48 \\ 1001.75 & 1010.03 & 1013.35 & 1029.78 & 1032.38 & 1034.70 \\ 1039.54 & 1040.74 & 1041.78 & 1053.10 & 1092.11 & 1140.02 \\ 1148.35 & 1149.87 & 1169.53 & 1182.43 & 1183.87 & 1260.78 \\ 1263.07 & 1271.49 & 1321.54 & 1327.83 & 1331.47 & 1341.85 \\ 1351.61 & 1362.49 & 1370.48 & 1374.41 & 1383.19 & 1391.99 \\ 1397.36 & 1469.87 & 1471.29 & 1472.75 & 1495.90 & 1500.11 \\ 1504.49 & 1524.79 & 1557.07 & 1559.60 & 1579.70 & 1588.44 \\ 1605.82 & 1612.73 & 1614.34 & 1630.48 & 1661.77 & 2550.93 \\ 3122.26 & 3122.43 & 3124.13 & 3127.64 & 3154.45 & 3154.75 \\ 3156.14 & 3156.91 & 3173.61 & 3173.88 & 3174.97 & 3175.93\end{array}$

TURBOMOLE Energies and Thermochemistry ${ }^{1}$ RI-PBE/TZVP,SV(P) $-3909.98022$

ZPE 0.3291547

RI-PBE/TZVP,SV(P) + ZPE RI-PBE/TZVPP $-3909.651065$ B3-LYP/TZVP,SV(P) $\mathrm{ZPE}(\mathrm{kJ} / \mathrm{mol})$ $-3912.282926$ $\ln$ (qtrans) $-3911.344085$

864.2 In(qrot) 20.24

In(qvib) 17.07 chem.pot. $(\mathrm{kJ} / \mathrm{mol})$ 31.14 energy $(\mathrm{kJ} / \mathrm{mol})$ 694.53

951.1 entropy $(\mathrm{kJ} / \mathrm{mol} / \mathrm{K})$ 0.86883 $\mathrm{Cv}(\mathrm{kJ} / \mathrm{mol}-\mathrm{K})$ 0.5382076 $\mathrm{Cp}((\mathrm{kJ} / \mathrm{mol}-\mathrm{K})$ 0.5465219 $\mathrm{RI}$ - PBE/TZVP, SV (P) $\left\langle\mathrm{S}^{2}\right\rangle$ 0.87 $\mathrm{RI}-\mathrm{PBE} / \mathrm{TZVPP}<\mathrm{S}^{2}>$ 0.87 B3 - LYP/TZVP, SV (P) $<\mathrm{S}^{2}>$ 1.35

${ }^{1}$ Freeh output; $298.15 \mathrm{~K}, 0.1 \mathrm{MPa}$, unscaled 
${ }^{2} \mathbf{A}$

\section{Chloropentafluorobenzene: $[($ Porphyrin $)(\mathrm{Fe}=$ O)(SH)] Product Complex (Cl-addition)}

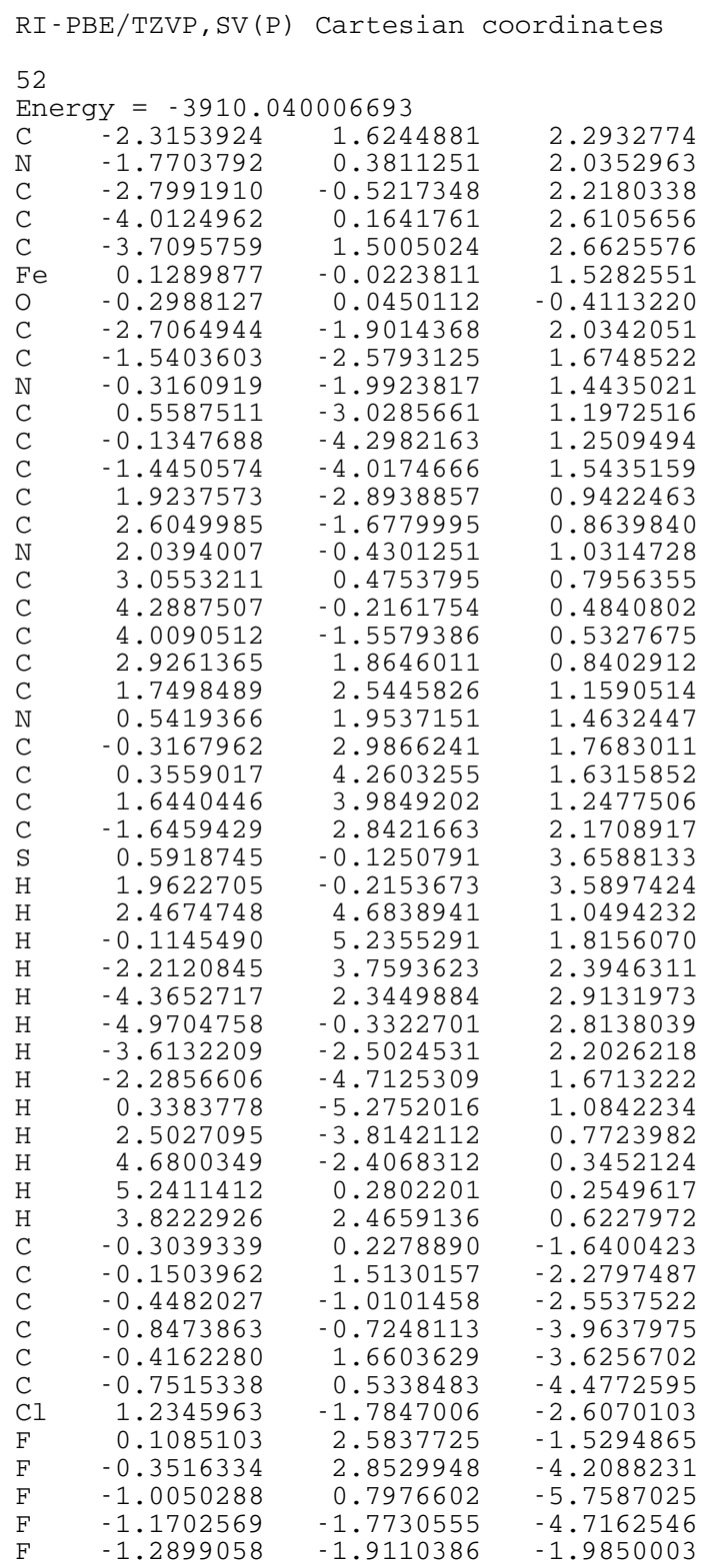

$\begin{array}{rrrrrr}0.00 & 0.00 & 0.00 & 0.00 & 0.00 & 0.00 \\ 8.14 & 15.89 & 17.78 & 34.98 & 45.94 & 53.40 \\ 62.34 & 70.92 & 84.30 & 102.45 & 105.56 & 116.00 \\ 126.43 & 138.65 & 145.85 & 147.74 & 165.26 & 175.29 \\ 218.41 & 221.24 & 230.51 & 239.71 & 254.56 & 258.89 \\ 266.44 & 272.13 & 274.28 & 277.20 & 279.04 & 284.43 \\ 290.28 & 311.75 & 316.50 & 320.92 & 340.60 & 351.81 \\ 357.77 & 360.10 & 360.74 & 394.06 & 400.44 & 403.08 \\ 411.56 & 411.83 & 419.43 & 420.15 & 420.79 & 422.06 \\ 433.94 & 459.72 & 492.12 & 563.12 & 603.30 & 618.21 \\ 642.29 & 652.90 & 657.50 & 658.11 & 673.56 & 685.10 \\ 695.53 & 696.85 & 697.88 & 700.11 & 719.68 & 724.50 \\ 734.89 & 736.11 & 762.28 & 769.33 & 770.58 & 770.84 \\ 776.94 & 777.72 & 784.73 & 785.30 & 786.16 & 799.16 \\ 835.40 & 843.87 & 844.73 & 852.19 & 884.78 & 889.42 \\ 890.27 & 892.35 & 986.13 & 991.94 & 1000.65 & 1002.11 \\ 1009.69 & 1011.88 & 1012.46 & 1030.66 & 1031.81 & 1035.25 \\ 1040.52 & 1041.34 & 1041.99 & 1053.95 & 1123.26 & 1139.21 \\ 1143.82 & 1145.33 & 1147.48 & 1182.59 & 1182.92 & 1247.09 \\ 1260.73 & 1262.54 & 1305.08 & 1328.40 & 1331.32 & 1334.26 \\ 1345.01 & 1361.53 & 1365.50 & 1376.26 & 1388.14 & 1389.07 \\ 1390.51 & 1442.54 & 1471.34 & 1473.01 & 1474.69 & 1507.08 \\ 1523.41 & 1558.23 & 1559.17 & 1579.10 & 1582.17 & 1612.01 \\ 1612.41 & 1620.81 & 1628.94 & 1660.47 & 1698.84 & 2515.72 \\ 3121.22 & 3121.74 & 3122.52 & 3125.06 & 3153.40 & 3153.46 \\ 3153.86 & 3155.40 & 3172.67 & 3172.70 & 3173.14 & 3174.72\end{array}$

TURBOMOLE Energies and Thermochemistry ${ }^{1}$ RI-PBE/TZVP,SV(P) $-3910.040007$

ZPE

0.3310848

RI-PBE/TZVP,SV(P) + ZPE

RI-PBE/TZVPP

$-3909.708922$

B3-LYP/TZVP,SV(P)

$\mathrm{ZPE}(\mathrm{kJ} / \mathrm{mol})$

$-3912.340692$

$-3911.422813$

869.3

$\ln$ (qtrans)

20.24

In(qrot)

17.27

In(qvib)

32.79

chem.pot. $(\mathrm{kJ} / \mathrm{mol})$

695.01

energy $(\mathrm{kJ} / \mathrm{mol})$

956.92

entropy $(\mathrm{kJ} / \mathrm{mol} / \mathrm{K})$

0.88675

$\mathrm{Cv}(\mathrm{kJ} / \mathrm{mol}-\mathrm{K})$

0.5406937

$\mathrm{Cp}((\mathrm{kJ} / \mathrm{mol}-\mathrm{K})$

RI - PBE/TZVP, SV (P) $\quad\left\langle\mathrm{S}^{2}>\right.$

0.549008

$\mathrm{RI}-\mathrm{PBE} / \mathrm{TZVPP}<\mathrm{S}^{2}>$

0.88

B3 - LYP/TZVP, SV (P) $<\mathrm{S}^{2}>$

0.89

0.81

${ }^{1}$ Freeh output; $298.15 \mathrm{~K}, 0.1 \mathrm{MPa}$, unscaled 


\section{2-Chloro-2,3,4,5,6-}

\section{pentafluorocyclohexadienone}

$\begin{array}{lrrr}13 & & \\ \text { Energy }=-1261.451975937 & \\ \text { C } & -0.2827783 & -1.1646602 & -0.1036851 \\ \text { C } & -0.3108660 & -0.4543096 & 1.2153316 \\ \text { C } & -0.0735578 & 0.8816067 & 1.2835579 \\ \text { C } & 0.0651824 & 1.6896678 & 0.0726262 \\ \text { C } & 0.0057985 & 1.1542854 & -1.1872609 \\ \text { C } & -0.3687547 & -0.2521230 & -1.3771611 \\ \text { F } & 0.0157437 & 1.5303777 & 2.4419814 \\ \text { F } & 0.2693822 & 2.9861234 & 0.2636402 \\ \text { F } & 0.0856430 & 1.9264033 & -2.2664827 \\ \text { F } & -0.4402025 & -1.2081373 & 2.3014689 \\ \text { F } & -1.2464543 & -2.1122302 & -0.1668229 \\ \text { O } & -0.7129075 & -0.7281249 & -2.4429331 \\ \text { Cl } & 1.3538015 & -1.9729762 & -0.2440459\end{array}$

Vibrational frequencies

$\begin{array}{rrrrrr}0.00 & 0.00 & 0.00 & 0.00 & 0.00 & 0.00 \\ 48.83 & 97.15 & 126.43 & 157.88 & 163.97 & 237.55 \\ 266.01 & 267.93 & 277.13 & 309.42 & 312.01 & 356.76 \\ 398.94 & 408.41 & 428.87 & 504.10 & 575.94 & 590.11 \\ 638.60 & 678.55 & 783.62 & 812.60 & 990.24 & 998.56 \\ 1110.42 & 1151.08 & 1251.36 & 1317.42 & 1354.44 & 1424.50 \\ 1641.10 & 1715.31 & 1757.41 & & & \end{array}$

TURBOMOLE Energies and Thermochemistry ${ }^{1}$

\begin{tabular}{lr}
\hline RI-PBE/TZVP,SV(P) & -1261.451976 \\
\hline ZPE & 0.0527456 \\
RI-PBE/TZVP,SV(P) + ZPE & -1261.39923 \\
RI-PBE/TZVPP & -1262.555005 \\
B3-LYP/TZVP,SV(P) & -1262.028322 \\
ZPE (kJ/mol) & 138.5 \\
In(qtrans) & 18.68 \\
In(qrot) & 14.35 \\
In(qvib) & 7.41 \\
chem.pot. (kJ/mol) & 38.21 \\
energy $(\mathrm{kJ} / \mathrm{mol})$ & 168.49 \\
entropy $(\mathrm{kJ} / \mathrm{mol} / \mathrm{K})$ & 0.44529 \\
Cv $(\mathrm{kJ} / \mathrm{mol}-\mathrm{K})$ & 0.1681613 \\
Cp $((\mathrm{kJ} / \mathrm{mol}-\mathrm{K})$ & 0.1764756 \\
\hline
\end{tabular}

${ }^{1}$ Freeh output; $298.15 \mathrm{~K}, 0.1 \mathrm{MPa}$, unscaled 
${ }^{4} \mathbf{A}$

Chloropentafluorobenzene: $[($ Porphyrin $)(\mathrm{Fe}=$ O)(SH)] Prereactant Complex (Cl-addition)

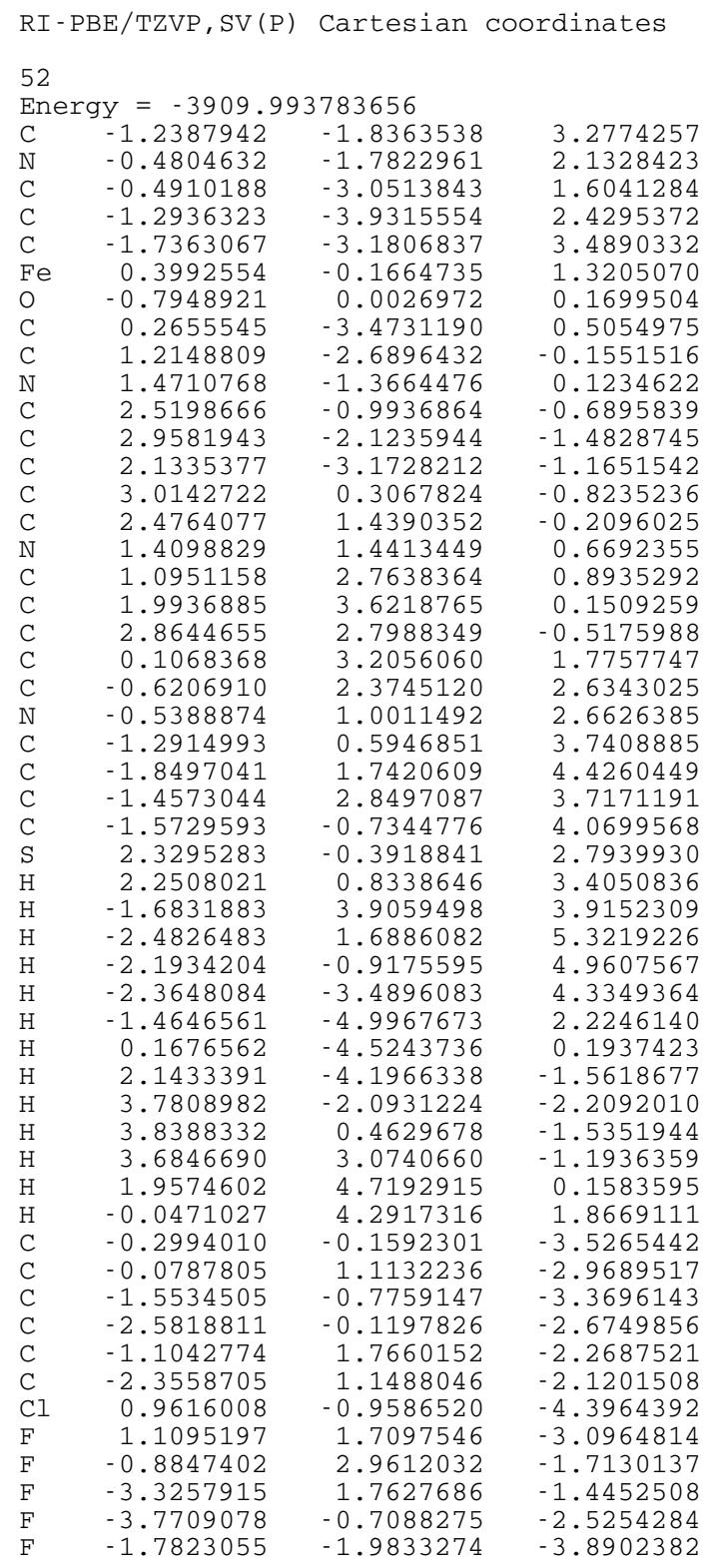

$\begin{array}{rrrrrr}i 11.27 & 0.00 & 0.00 & 0.00 & 0.00 & 0.00 \\ 0.00 & 11.76 & 14.49 & 26.39 & 32.94 & 49.48 \\ 58.30 & 62.18 & 88.02 & 90.41 & 103.16 & 109.48 \\ 133.42 & 141.73 & 147.32 & 158.27 & 173.44 & 175.73 \\ 180.30 & 192.68 & 209.89 & 212.23 & 219.51 & 234.32 \\ 261.31 & 262.70 & 267.18 & 270.94 & 272.06 & 279.69 \\ 287.05 & 298.66 & 300.90 & 317.39 & 318.19 & 343.50 \\ 350.97 & 359.13 & 360.92 & 370.15 & 377.42 & 385.71 \\ 404.05 & 407.78 & 419.64 & 421.38 & 422.19 & 424.55 \\ 428.99 & 447.24 & 458.59 & 506.93 & 551.20 & 568.95 \\ 605.10 & 620.89 & 650.14 & 653.35 & 654.01 & 667.96 \\ 673.84 & 692.00 & 693.01 & 697.25 & 713.73 & 723.82 \\ 733.89 & 734.86 & 753.31 & 763.12 & 769.80 & 770.02 \\ 775.50 & 778.65 & 785.96 & 786.51 & 800.18 & 810.99 \\ 843.00 & 851.55 & 853.17 & 861.27 & 870.47 & 887.28 \\ 892.56 & 892.87 & 895.22 & 994.79 & 997.61 & 1004.09 \\ 1005.45 & 1010.67 & 1017.37 & 1030.81 & 1033.39 & 1036.67 \\ 1040.33 & 1041.24 & 1043.36 & 1055.53 & 1107.95 & 1138.05 \\ 1147.09 & 1149.55 & 1172.95 & 1182.57 & 1185.04 & 1261.70 \\ 1263.06 & 1292.97 & 1294.56 & 1326.09 & 1327.07 & 1338.96 \\ 1359.21 & 1363.20 & 1371.71 & 1380.75 & 1385.37 & 1395.61 \\ 1444.48 & 1464.40 & 1467.51 & 1468.03 & 1499.91 & 1521.12 \\ 1523.05 & 1524.26 & 1552.89 & 1553.61 & 1572.76 & 1580.53 \\ 1601.47 & 1602.65 & 1637.44 & 1638.33 & 1654.33 & 2552.97 \\ 3124.93 & 3125.37 & 3127.31 & 3129.94 & 3156.29 & 3156.44 \\ 3157.83 & 3158.08 & 3175.08 & 3175.24 & 3176.62 & 3176.86\end{array}$

TURBOMOLE Energies and Thermochemistry ${ }^{1}$ RI-PBE/TZVP,SV(P) $-3909.993784$

ZPE

0.3293788

RI-PBE/TZVP,SV(P) + ZPE

RI-PBE/TZVPP

$-3909.664465$

B3-LYP/TZVP,SV(P)

$\mathrm{ZPE}(\mathrm{kJ} / \mathrm{mol})$

$-3912.300308$

$-3911.374965$

$\ln$ (qtrans)

866

In(qrot)

20.24

In(qvib)

17.28

chem.pot. $(\mathrm{kJ} / \mathrm{mol})$

33.1

energy $(\mathrm{kJ} / \mathrm{mol})$

690.92

955.25

entropy $(\mathrm{kJ} / \mathrm{mol} / \mathrm{K})$

0.89487

$\mathrm{Cv}(\mathrm{kJ} / \mathrm{mol}-\mathrm{K})$

0.5447575

$\mathrm{Cp}((\mathrm{kJ} / \mathrm{mol}-\mathrm{K})$

0.5530718

$\mathrm{RI}-\mathrm{PBE} / \mathrm{TZVP}, \mathrm{SV}(\mathrm{P}) \quad<\mathrm{S}^{2}>$

3.77

$\mathrm{RI}-\mathrm{PBE} / \mathrm{TZVPP}<\mathrm{S}^{2}>$

3.77

B3 - LYP / TZVP, SV (P) $\quad<\mathrm{S}^{2}>$

3.79

${ }^{1}$ Freeh output; $298.15 \mathrm{~K}, 0.1 \mathrm{MPa}$, unscaled 
${ }^{4} \mathbf{A}$

\section{Chloropentafluorobenzene: $[($ Porphyrin $)(\mathrm{Fe}=$ O)(SH)] Transition State (Cl-addition)}

RI-PBE/TZVP, SV (P) Cartesian coordinates

$$
52
$$$$
\text { Energy }=-3909.968017566
$$

\begin{tabular}{|c|c|c|c|}
\hline & & & \\
\hline C & -3.1755861 & -1.1559444 & 0.2283530 \\
\hline $\mathrm{N}$ & -1.8609966 & -1.3990668 & 0.5481374 \\
\hline C & -1.6452635 & -2.7364674 & 0.3062807 \\
\hline $\mathrm{C}$ & -2.8565977 & -3.3560880 & -0.1937665 \\
\hline C & -3.8173122 & -2.3767714 & -0.2174711 \\
\hline $\mathrm{Fe}$ & -0.5015556 & -0.0540118 & 1.1679585 \\
\hline $\mathrm{O}$ & 0.1009533 & 0.0845692 & -0.4632117 \\
\hline $\mathrm{C}$ & -0.4657370 & -3.4262983 & 0.6003192 \\
\hline C & 0.6267032 & -2.8780363 & 1.2798642 \\
\hline $\mathrm{N}$ & 0.7431549 & -1.5598326 & 1.6518402 \\
\hline $\mathrm{C}$ & 1.9047622 & -1.4645862 & 2.3851219 \\
\hline C & 2.5330822 & -2.7646952 & 2.4932982 \\
\hline $\mathrm{C}$ & 1.7502402 & -3.6406662 & 1.7836616 \\
\hline C & 2.4611839 & -0.2746773 & 2.8629955 \\
\hline $\mathrm{C}$ & 1.9642855 & 1.0045884 & 2.5994713 \\
\hline $\mathrm{N}$ & 0.8071935 & 1.2818296 & 1.904550 \\
\hline $\mathrm{C}$ & 0.7601614 & 2.6487296 & 1.7598302 \\
\hline $\mathrm{C}$ & 1.9227457 & 3.2568072 & 2.3735597 \\
\hline C & 2.6592625 & 2.2345205 & 2.9182234 \\
\hline $\mathrm{C}$ & -0.3050305 & 3.3563214 & 1.1946157 \\
\hline C & -1.5197384 & 2.7829567 & 0.8073463 \\
\hline $\mathrm{N}$ & -1.7976974 & 1.4335705 & 0.8182054 \\
\hline C & -3.1225666 & 1.3099254 & 0.4627962 \\
\hline C & -3.7056712 & 2.6177991 & 0.2450774 \\
\hline $\mathrm{C}$ & -2.7005029 & 3.5334115 & 0.4331527 \\
\hline C & -3.7870271 & 0.1010789 & 0.2378348 \\
\hline S & -1.2953560 & -0.2661254 & 3.3613224 \\
\hline $\mathrm{H}$ & -1.4758568 & 1.0691490 & 3.619398 \\
\hline $\mathrm{H}$ & -2.7447180 & 4.6274709 & 0.3487427 \\
\hline $\mathrm{H}$ & -4.7507850 & 2.7963894 & -0.0412928 \\
\hline $\mathrm{H}$ & -4.8500066 & 0.1514309 & -0.0438248 \\
\hline $\mathrm{H}$ & -4.8680126 & -2.4536154 & -0.5274110 \\
\hline $\mathrm{H}$ & -2.9505272 & -4.4158542 & -0.4659167 \\
\hline $\mathrm{H}$ & -0.4304093 & -4.5001723 & 0.3612751 \\
\hline $\mathrm{H}$ & 1.8963568 & -4.7174207 & 1.6247982 \\
\hline $\mathrm{H}$ & 3.4690987 & -2.9635499 & 3.0320816 \\
\hline $\mathrm{H}$ & 3.4055825 & -0.3456457 & 3.4241356 \\
\hline $\mathrm{H}$ & 3.6058493 & 2.2915897 & 3.4720775 \\
\hline $\mathrm{H}$ & 2.1263695 & 4.3357735 & 2.3950207 \\
\hline $\mathrm{H}$ & -0.2164993 & 4.4519692 & 1.1364597 \\
\hline C & 1.5833278 & 0.1152387 & -1.5953358 \\
\hline C & 1.3044221 & 1.3798598 & -2.2252278 \\
\hline C & 1.3117315 & -1.0640125 & -2.3733823 \\
\hline C & 0.5576865 & -0.9931292 & -3.5418584 \\
\hline C & 0.5484698 & 1.4496631 & -3.3928346 \\
\hline $\mathrm{C}$ & 0.199671 & 0.2642848 & -4.0745099 \\
\hline $\mathrm{Cl}$ & 2.9780951 & 0.0522628 & -0.5374385 \\
\hline $\mathrm{F}$ & 1.6570555 & 2.5012978 & -1.5975071 \\
\hline $\mathrm{F}$ & 0.1993958 & 2.6319178 & -3.9084455 \\
\hline $\mathrm{F}$ & -0.5066935 & 0.3278138 & -5.2027324 \\
\hline $\mathrm{F}$ & 0.2253351 & -2.1032085 & -4.206659 \\
\hline $\mathrm{F}$ & 1.6803 & -2.2528970 & -1.895721 \\
\hline
\end{tabular}

$\begin{array}{rrrrrr}\text { i535.47 } & 0.00 & 0.00 & 0.00 & 0.00 & 0.00 \\ 0.00 & 13.18 & 21.37 & 28.78 & 50.59 & 53.25 \\ 57.68 & 61.67 & 76.79 & 96.53 & 101.76 & 119.81 \\ 127.01 & 130.69 & 143.45 & 150.74 & 171.08 & 171.65 \\ 189.24 & 208.64 & 211.47 & 223.74 & 239.79 & 245.89 \\ 259.41 & 261.06 & 262.81 & 273.65 & 275.87 & 286.00 \\ 295.38 & 297.21 & 299.12 & 301.50 & 322.90 & 340.45 \\ 350.66 & 360.34 & 362.02 & 367.27 & 380.88 & 383.65 \\ 412.16 & 413.33 & 419.72 & 421.55 & 422.67 & 422.94 \\ 423.99 & 458.84 & 475.74 & 498.15 & 565.84 & 607.44 \\ 609.75 & 619.55 & 651.68 & 655.09 & 656.13 & 670.47 \\ 675.01 & 684.18 & 693.99 & 694.80 & 698.29 & 717.17 \\ 724.29 & 734.02 & 735.29 & 750.97 & 762.95 & 769.99 \\ 770.18 & 775.94 & 780.56 & 785.41 & 785.86 & 800.06 \\ 839.42 & 841.40 & 849.10 & 851.20 & 858.28 & 886.32 \\ 891.39 & 892.05 & 893.83 & 991.91 & 996.06 & 1005.23 \\ 1005.52 & 1011.00 & 1017.55 & 1032.07 & 1032.73 & 1035.47 \\ 1041.11 & 1041.42 & 1042.69 & 1055.71 & 1088.68 & 1139.41 \\ 1146.75 & 1148.83 & 1162.98 & 1181.18 & 1184.14 & 1261.18 \\ 1263.17 & 1267.36 & 1308.69 & 1327.44 & 1328.18 & 1338.53 \\ 1345.50 & 1356.02 & 1363.72 & 1378.47 & 1384.18 & 1388.35 \\ 1390.99 & 1465.47 & 1467.33 & 1468.51 & 1491.39 & 1498.12 \\ 1501.08 & 1521.04 & 1553.01 & 1553.62 & 1573.79 & 1582.37 \\ 1589.18 & 1600.88 & 1602.68 & 1622.39 & 1653.44 & 2548.49 \\ 3123.67 & 3123.75 & 3125.11 & 3125.25 & 3154.83 & 3154.99 \\ 3155.58 & 3155.70 & 3173.66 & 3173.84 & 3174.43 & 3174.59\end{array}$

TURBOMOLE Energies and Thermochemistry ${ }^{1}$ RI-PBE/TZVP,SV(P) $-3909.968018$

ZPE 0.3287223

RI-PBE/TZVP,SV(P) + ZPE RI-PBE/TZVPP

B3-LYP/TZVP,SV(P)

$\mathrm{ZPE}(\mathrm{kJ} / \mathrm{mol})$

909.639295

In(qtrans)

$-3912.271722$

$-3911.316434$

863.1

In(qrot)

20.24

In(qvib)

17.17

30.56

chem.pot. $(\mathrm{kJ} / \mathrm{mol})$

694.56

energy $(\mathrm{kJ} / \mathrm{mol})$

949.99

entropy $(\mathrm{kJ} / \mathrm{mol} / \mathrm{K})$

0.86503

$\mathrm{Cv}(\mathrm{kJ} / \mathrm{mol}-\mathrm{K})$

$\mathrm{Cp}((\mathrm{kJ} / \mathrm{mol}-\mathrm{K})$

$\mathrm{RI}-\mathrm{PBE} / \mathrm{TZVP}, \mathrm{SV}(\mathrm{P}) \quad\left\langle\mathrm{S}^{2}>\right.$

0.5387404

0.5470547

$\mathrm{RI}-\mathrm{PBE} / \mathrm{TZVPP}<\mathrm{S}^{2}>$

3.77

3.77

B3 - LYP/TZVP, SV (P) $<\mathrm{S}^{2}>$

4.98

${ }^{1}$ Freeh output; $298.15 \mathrm{~K}, 0.1 \mathrm{MPa}$, unscaled 
${ }^{4} \mathbf{A}$

\section{Chloropentafluorobenzene: $[($ Porphyrin $)(\mathrm{Fe}=$ O)(SH)] Product Complex (Cl-addition)}

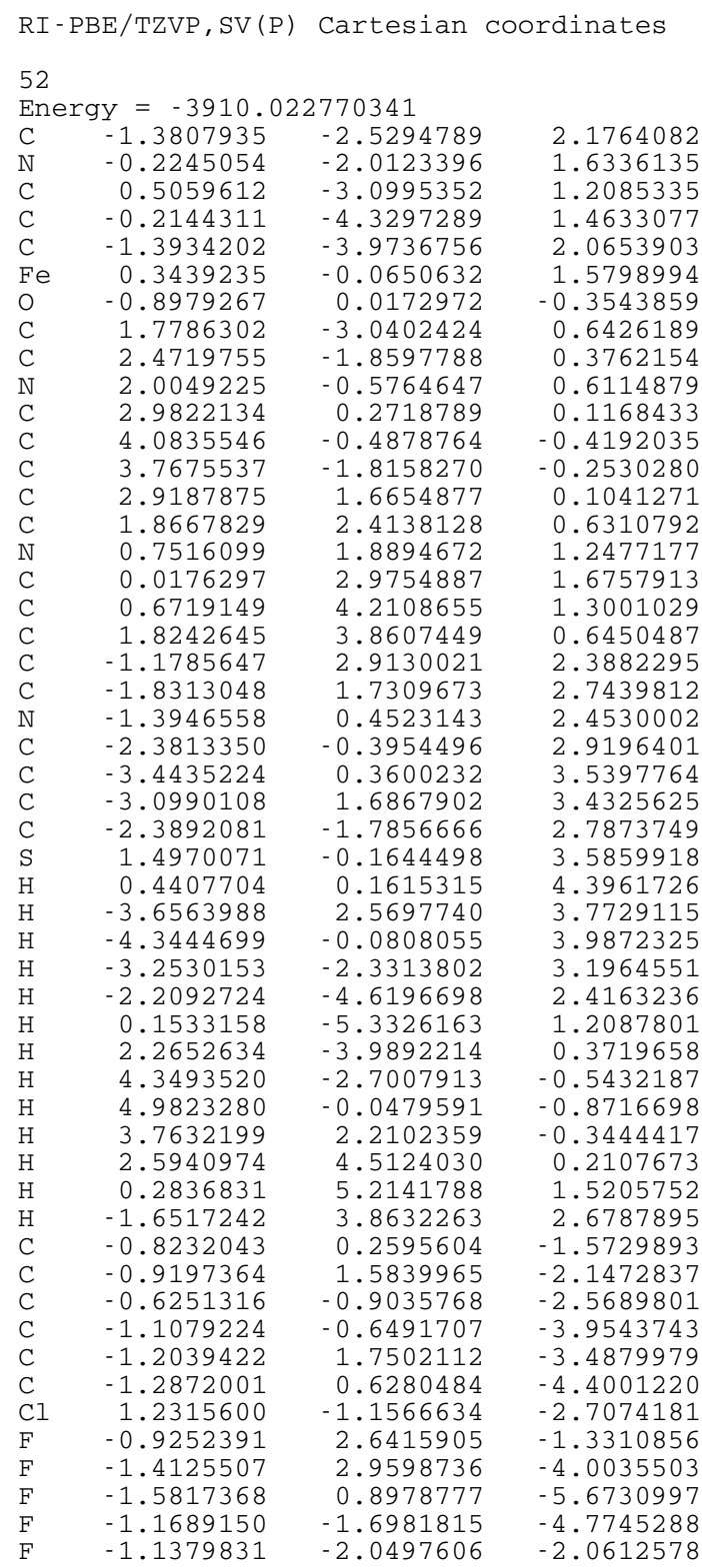

$\begin{array}{rrrrrr}0.00 & 0.00 & 0.00 & 0.00 & 0.00 & 0.00 \\ 10.58 & 22.83 & 33.16 & 38.23 & 44.96 & 48.22 \\ 50.38 & 57.44 & 80.15 & 85.38 & 88.94 & 91.93 \\ 115.59 & 141.12 & 144.37 & 146.84 & 163.67 & 173.60 \\ 196.76 & 209.35 & 210.17 & 228.27 & 242.83 & 252.19 \\ 255.93 & 261.85 & 265.96 & 271.98 & 276.83 & 279.63 \\ 283.25 & 289.91 & 308.91 & 310.03 & 316.49 & 337.79 \\ 345.98 & 351.72 & 354.56 & 357.01 & 391.19 & 403.22 \\ 405.10 & 406.03 & 416.13 & 417.48 & 421.53 & 422.62 \\ 429.54 & 456.83 & 475.95 & 552.68 & 553.52 & 592.24 \\ 626.89 & 640.09 & 652.23 & 656.24 & 659.61 & 673.99 \\ 685.00 & 696.74 & 699.14 & 699.87 & 718.97 & 725.77 \\ 733.01 & 737.74 & 763.85 & 772.42 & 773.41 & 775.13 \\ 780.55 & 782.61 & 782.88 & 784.89 & 787.46 & 799.03 \\ 835.56 & 844.81 & 845.99 & 853.73 & 890.20 & 893.99 \\ 895.32 & 897.44 & 983.47 & 991.63 & 999.79 & 1001.15 \\ 1005.43 & 1011.34 & 1014.61 & 1030.09 & 1031.78 & 1036.35 \\ 1040.70 & 1041.13 & 1042.17 & 1052.31 & 1117.19 & 1139.40 \\ 1143.59 & 1145.79 & 1152.34 & 1181.61 & 1183.07 & 1251.56 \\ 1260.27 & 1261.40 & 1294.36 & 1326.48 & 1329.04 & 1336.85 \\ 1348.22 & 1356.67 & 1366.76 & 1368.29 & 1375.88 & 1382.34 \\ 1388.28 & 1444.70 & 1465.19 & 1469.62 & 1478.80 & 1509.60 \\ 1520.57 & 1549.34 & 1559.93 & 1565.19 & 1579.55 & 1600.52 \\ 1613.71 & 1616.08 & 1622.44 & 1664.27 & 1690.72 & 2560.81 \\ 3124.84 & 3125.29 & 3126.32 & 3128.34 & 3154.50 & 3155.33 \\ 3155.51 & 3156.33 & 3173.15 & 3174.43 & 3174.66 & 3174.92\end{array}$

TURBOMOLE Energies and Thermochemistry ${ }^{1}$ RI-PBE/TZVP,SV(P)

$-3910.02277$

ZPE

0.3298319

RI-PBE/TZVP,SV(P) + ZPE

RI-PBE/TZVPP

$-3909.692939$

B3-LYP/TZVP,SV(P)

$-3912.32527$

$\mathrm{ZPE}(\mathrm{kJ} / \mathrm{mol})$

$-3911.402773$

$\ln$ (qtrans)

866

In(qrot)

20.24

In(qvib)

17.28

33.1

chem.pot. $(\mathrm{kJ} / \mathrm{mol})$

690.92

energy $(\mathrm{kJ} / \mathrm{mol})$

955.25

entropy $(\mathrm{kJ} / \mathrm{mol} / \mathrm{K})$

0.89487

$\mathrm{Cv}(\mathrm{kJ} / \mathrm{mol}-\mathrm{K})$

0.5447575

$\mathrm{Cp}((\mathrm{kJ} / \mathrm{mol}-\mathrm{K})$

$\mathrm{RI}$ - PBE/TZVP, SV (P) $\left\langle\mathrm{S}^{2}>\right.$

0.5530718

$\mathrm{RI}-\mathrm{PBE} / \mathrm{TZVPP}<\mathrm{S}^{2}>$

3.80

B3 - LYP / TZVP, SV (P) $<\mathrm{S}^{2}>$

3.80

3.88

${ }^{1}$ Freeh output; $298.15 \mathrm{~K}, 0.1 \mathrm{MPa}$, unscaled 
${ }^{2} \mathbf{A}$

Chloropentafluorobenzene: $[($ Porphyrin $)(\mathrm{Fe}=$ O)(SH)] Prereactant Complex ( $p$-F-addition)

RI-PBE/TZVP, SV (P) Cartesian coordinates

52

Energy $=-3910.000430413$

\begin{tabular}{|c|c|c|c|}
\hline $\mathrm{C}$ & -0.9610302 & 2.8283278 & 0.5189277 \\
\hline $\mathrm{N}$ & -1.1842666 & 1.4904971 & 0.2742692 \\
\hline C & -1.8900845 & 1.4281045 & -0.9021966 \\
\hline C & -2.1289943 & 2.7593890 & -1.4199557 \\
\hline $\mathrm{C}$ & -1.5420972 & 3.6309759 & -0.5381538 \\
\hline $\mathrm{Fe}$ & -0.4512478 & -0.0786279 & 1.3450381 \\
\hline $\mathrm{O}$ & 1.0276011 & 0.1267203 & 0.6693496 \\
\hline $\mathrm{C}$ & -2.2836031 & 0.2463912 & -1.5355202 \\
\hline C & -1.9498539 & -1.0389084 & -1.1058491 \\
\hline $\mathrm{N}$ & -1.2160841 & -1.3481400 & 0.0218140 \\
\hline $\mathrm{C}$ & -1.0921390 & -2.7223857 & 0.0283582 \\
\hline $\mathrm{C}$ & -1.7505652 & -3.2921667 & -1.1270384 \\
\hline $\mathrm{C}$ & -2.2951689 & -2.2458866 & -1.8262050 \\
\hline C & -0.4583878 & -3.4728999 & 1.0187755 \\
\hline C & 0.1026568 & -2.9446788 & 2.1835801 \\
\hline $\mathrm{N}$ & 0.1434066 & -1.6079837 & 2.5071191 \\
\hline C & 0.7803044 & -1.5313160 & 3.7285735 \\
\hline C & 1.1260055 & -2.8575016 & 4.1965845 \\
\hline C & 0.7125209 & -3.7378001 & 3.2293880 \\
\hline C & 1.0960293 & -0.3586808 & 4.4210413 \\
\hline C & 0.8510300 & 0.9382344 & 3.9658530 \\
\hline $\mathrm{N}$ & 0.2186961 & 1.2492700 & 2.7855324 \\
\hline C & 0.2342176 & 2.6221319 & 2.6856470 \\
\hline C & 0.8750387 & 3.1978643 & 3.8515355 \\
\hline $\mathrm{C}$ & 1.2526062 & 2.1500354 & 4.6526085 \\
\hline C & -0.2994656 & 3.3607095 & 1.6283037 \\
\hline S & -2.4427006 & 0.2005293 & 2.550759 \\
\hline $\mathrm{H}$ & -2.0738935 & -0.4440981 & 3.70595 \\
\hline $\mathrm{H}$ & 1.7679162 & 2.1781492 & 5.6220 \\
\hline $\mathrm{H}$ & 1.0099345 & 4.2743402 & 4.02215 \\
\hline $\mathrm{H}$ & -0.2059040 & 4.4562103 & 1.6799565 \\
\hline $\mathrm{H}$ & -1.5016382 & 4.7274638 & -0.58105 \\
\hline $\mathrm{H}$ & -2.6673633 & 2.9807212 & -2.3506989 \\
\hline $\mathrm{H}$ & -2.8541688 & 0.3348525 & -2.4720029 \\
\hline $\mathrm{H}$ & -2.8652059 & -2.2685220 & -2.7638614 \\
\hline $\mathrm{H}$ & -1.7876646 & -4.3652676 & -1.3565737 \\
\hline $\mathrm{H}$ & -0.4241946 & -4.5649803 & 0.8862211 \\
\hline $\mathrm{H}$ & 0.8026628 & -4.8319837 & 3.2110642 \\
\hline $\mathrm{H}$ & 1.6378210 & -3.0692312 & 5.1448640 \\
\hline $\mathrm{H}$ & 1.6127295 & -0.4681128 & 3869211 \\
\hline $\mathrm{C}$ & 2.3950613 & 0.3848589 & -1.8151125 \\
\hline C & 1.7040906 & 1.4439361 & -2.427549 \\
\hline C & 2.0553549 & -0.9430840 & -2.12519 \\
\hline C & 1.0672098 & -1.2107773 & -3.08657 \\
\hline C & 0.7121174 & 1.1755918 & -3.3843392 \\
\hline C & 0.3885199 & -0.1525032 & -3.7171206 \\
\hline $\mathrm{F}$ & 1.9965192 & 2.7054459 & -2.1047230 \\
\hline $\mathrm{F}$ & 0.0650953 & 2.1926536 & -3.9585724 \\
\hline $\mathrm{F}$ & 0.7638256 & -2.4773140 & -3.3755906 \\
\hline $\mathrm{F}$ & 2.6839284 & -1.9490247 & -1.5171832 \\
\hline$F$ & 3.3552683 & 0.6373340 & -0.933612 \\
\hline $\mathrm{Cl}$ & -0.83416 & -0.4775125 & -4.896950 \\
\hline
\end{tabular}

$\begin{array}{rrrrrr}i 10.38 & 0.00 & 0.00 & 0.00 & 0.00 & 0.00 \\ 0.00 & 11.94 & 17.34 & 21.55 & 32.06 & 44.12 \\ 55.77 & 65.06 & 95.57 & 98.95 & 100.40 & 117.75 \\ 131.85 & 135.24 & 147.45 & 152.38 & 157.97 & 180.45 \\ 192.03 & 206.34 & 212.08 & 213.60 & 230.67 & 238.83 \\ 260.03 & 261.09 & 271.18 & 275.01 & 276.64 & 280.19 \\ 287.91 & 298.11 & 298.95 & 299.94 & 312.46 & 346.14 \\ 350.63 & 358.86 & 360.35 & 366.16 & 377.83 & 385.39 \\ 397.74 & 402.60 & 418.67 & 418.82 & 420.91 & 422.25 \\ 429.10 & 443.90 & 459.33 & 507.09 & 570.57 & 579.24 \\ 603.33 & 620.19 & 649.45 & 654.99 & 656.61 & 671.76 \\ 678.64 & 693.75 & 694.30 & 696.73 & 715.51 & 724.02 \\ 734.10 & 735.28 & 751.49 & 763.13 & 770.97 & 771.48 \\ 777.39 & 781.86 & 784.91 & 785.02 & 798.88 & 839.03 \\ 848.50 & 850.85 & 852.81 & 858.92 & 869.78 & 886.73 \\ 891.66 & 892.56 & 894.44 & 993.53 & 997.82 & 1002.28 \\ 1002.92 & 1011.29 & 1015.34 & 1032.30 & 1032.72 & 1036.43 \\ 1041.37 & 1041.60 & 1043.31 & 1054.52 & 1109.26 & 1140.26 \\ 1148.71 & 1149.16 & 1175.28 & 1183.42 & 1183.72 & 1261.67 \\ 1263.00 & 1302.79 & 1313.80 & 1328.96 & 1330.62 & 1340.82 \\ 1354.99 & 1363.50 & 1376.15 & 1382.61 & 1391.36 & 1392.71 \\ 1443.65 & 1468.87 & 1470.99 & 1472.30 & 1503.93 & 1521.35 \\ 1522.29 & 1525.04 & 1556.94 & 1557.61 & 1578.32 & 1601.59 \\ 1607.76 & 1609.63 & 1632.27 & 1633.81 & 1661.06 & 2539.89 \\ 3123.31 & 3124.54 & 3125.87 & 3131.57 & 3155.06 & 3156.00 \\ 3158.65 & 3159.77 & 3173.90 & 3174.98 & 3177.65 & 3179.16\end{array}$

TURBOMOLE Energies and Thermochemistry ${ }^{1}$ RI-PBE/TZVP,SV(P) $-3910.00043$

ZPE

0.3297455

RI-PBE/TZVP,SV(P) + ZPE

RI-PBE/TZVPP

$-3909.670685$

B3-LYP/TZVP,SV(P)

$\mathrm{ZPE}(\mathrm{kJ} / \mathrm{mol})$

$-3912.306296$

$-3911.361526$

865.7

$\ln$ (qtrans)

20.24

In(qrot)

17.22

In(qvib)

31.56

chem.pot. $(\mathrm{kJ} / \mathrm{mol})$

694.66

energy $(\mathrm{kJ} / \mathrm{mol})$

952.85

entropy $(\mathrm{kJ} / \mathrm{mol} / \mathrm{K})$

0.8743

$\mathrm{Cv}(\mathrm{kJ} / \mathrm{mol}-\mathrm{K})$

0.5377351

$\mathrm{Cp}((\mathrm{kJ} / \mathrm{mol}-\mathrm{K})$

0.5460494

RI - PBE/TZVP, SV (P) $\quad\left\langle\mathrm{S}^{2}>\right.$

1.12

$\mathrm{RI}-\mathrm{PBE} / \mathrm{TZVPP}<\mathrm{S}^{2}>$

1.12

B3 - LYP / TZVP, SV (P) $\quad<\mathrm{S}^{2}>$

1.52

${ }^{1}$ Freeh output; $298.15 \mathrm{~K}, 0.1 \mathrm{MPa}$, unscaled 
${ }^{2} \mathbf{A}$

\section{Chloropentafluorobenzene: $[($ Porphyrin $)(\mathrm{Fe}=$ O)(SH)] Transition State ( $p$-F-addition)}

\begin{tabular}{|c|c|c|c|}
\hline \multicolumn{2}{|c|}{ RI - PBE/TZVP, SV (P) } & Cartesian & coordinates \\
\hline & & & \\
\hline & $y=-3909.98$ & 576327 & \\
\hline $\mathrm{C}$ & -0.8162466 & 2.9297642 & 0.4392098 \\
\hline & -1.1035195 & 1.6238249 & 0.0917137 \\
\hline & -1.8328528 & 1.6909515 & -1.0769320 \\
\hline & -2.0153887 & 3.0679713 & -1.4769422 \\
\hline $\mathrm{C}$ & -1.3703382 & 3.8373307 & -0.5413741 \\
\hline $\mathrm{Fe}$ & -0.5486496 & -0.0271204 & 1.0762923 \\
\hline $\mathrm{O}$ & 1.0697400 & -0.0131078 & 0.5654619 \\
\hline & -2.2737579 & 0.5873165 & -1.8093870 \\
\hline & -2.0057138 & -0.7430172 & -1.4800637 \\
\hline & -1.3127601 & -1.1648244 & -0.3655466 \\
\hline c & -1.2823062 & -2.5453374 & -0.4374913 \\
\hline & -1.9399525 & -2.9979329 & -1.6446358 \\
\hline & -2.4008707 & -1.8775740 & -2.2871273 \\
\hline $\mathrm{C}$ & -0.7588578 & -3.3983892 & 0.5328634 \\
\hline $\mathrm{C}$ & -0.2402501 & -2.9883411 & 1.7653565 \\
\hline $\mathrm{N}$ & -0.1322725 & -1.6844348 & 2.1819300 \\
\hline $\mathrm{C}$ & 0.4376191 & -1.7284256 & 3.4362685 \\
\hline $\mathrm{C}$ & 0.6603515 & -3.1033563 & 3.8353442 \\
\hline $\mathrm{C}$ & 0.2473175 & -3.8891580 & 2.7891885 \\
\hline $\mathrm{C}$ & 0.8123432 & -0.6245523 & 4.2078304 \\
\hline $\mathrm{C}$ & 0.7178243 & 0.7092684 & 3.8 \\
\hline $\mathrm{N}$ & 0.1847461 & 1.1407247 & 2.6 \\
\hline C & 0.3259050 & 2.5055166 & 2.6 \\
\hline C & 0.964 & 2.95 & 3.82 \\
\hline $\mathrm{C}$ & 1.1987408 & 1.8400976 & 4.57 \\
\hline C & -0.1221067 & 3.3445440 & 1.5780234 \\
\hline $\mathrm{S}$ & -2.5554148 & 0.2078873 & 2.1809040 \\
\hline $\mathrm{H}$ & -2.4068200 & -0.7842166 & 3.1174798 \\
\hline $\mathrm{H}$ & 1.6630431 & 1.7671268 & 5.5716741 \\
\hline $\mathrm{H}$ & 1.1883795 & 4.0077678 & 4.0595718 \\
\hline $\mathrm{H}$ & 0.0454211 & 4.4257147 & 1.6984725 \\
\hline $\mathrm{H}$ & -1.2740660 & 4.9303915 & -0.5004722 \\
\hline $\mathrm{H}$ & -2.5561120 & 3.3896656 & -2.3766329 \\
\hline $\mathrm{H}$ & -2.8398577 & 0.7802662 & -2.7327354 \\
\hline $\mathrm{H}$ & -2.95 & -1.8066146 & -3.2326347 \\
\hline $\mathrm{H}$ & -2.03 & -4.05 & -1.94 \\
\hline $\mathrm{H}$ & -0.79 & -4.47 & 0.3298277 \\
\hline $\mathrm{H}$ & 0.26 & -4.9837180 & 2.7026790 \\
\hline $\mathrm{H}$ & 1.09 & -3.4108232 & 4.7946388 \\
\hline $\mathrm{H}$ & 1.2601810 & -0.8273122 & 5.1929555 \\
\hline $\mathrm{C}$ & 2.2247017 & 0.0220085 & -0.9039173 \\
\hline $\mathrm{C}$ & 1.8530910 & 1.2409223 & -1.5626736 \\
\hline $\mathrm{C}$ & 1.9309415 & -1.2037661 & -1.5885395 \\
\hline $\mathrm{C}$ & 1.1473908 & -1.2148881 & -2.7424711 \\
\hline C & 1.0563325 & 1.2234605 & -2.7051015 \\
\hline C & 0.7325083 & -0.0014466 & -3.3326252 \\
\hline $\mathrm{F}$ & 3287 & 2.3968014 & -1.0016391 \\
\hline $\mathrm{F}$ & 0.64 & 2.3750784 & -3.2408835 \\
\hline $\mathrm{F}$ & 26132 & -2.3793557 & -3.3182063 \\
\hline $\mathrm{F}$ & 2.35 & -2.3471095 & -1.0588949 \\
\hline $\mathrm{F}$ & 3.3175897 & 0.0456542 & -0.1306206 \\
\hline $\mathrm{Cl}$ & -0.1840315 & -0.0130962 & -4.7913014 \\
\hline
\end{tabular}

$\begin{array}{rrrrrr}1407.01 & 0.00 & 0.00 & 0.00 & 0.00 & 0.00 \\ 0.00 & 13.43 & 31.14 & 39.26 & 48.33 & 50.00 \\ 67.17 & 69.71 & 86.92 & 91.58 & 105.55 & 115.83 \\ 129.38 & 137.36 & 149.72 & 152.54 & 153.94 & 190.80 \\ 193.19 & 211.52 & 216.13 & 227.29 & 230.11 & 255.52 \\ 257.19 & 257.50 & 268.49 & 272.52 & 277.35 & 281.38 \\ 286.54 & 290.38 & 297.50 & 298.62 & 320.97 & 343.15 \\ 350.93 & 359.11 & 363.01 & 373.88 & 389.11 & 394.91 \\ 396.63 & 405.10 & 418.49 & 420.23 & 421.27 & 422.24 \\ 423.20 & 459.16 & 497.06 & 522.88 & 574.29 & 594.56 \\ 620.31 & 641.82 & 649.64 & 655.08 & 657.17 & 672.00 \\ 679.74 & 693.91 & 694.70 & 696.42 & 716.83 & 723.38 \\ 734.09 & 734.83 & 752.85 & 762.72 & 765.28 & 770.98 \\ 771.35 & 777.53 & 782.60 & 784.61 & 785.28 & 798.95 \\ 836.34 & 846.08 & 848.75 & 855.82 & 875.78 & 886.96 \\ 891.87 & 892.61 & 894.10 & 993.06 & 994.56 & 1001.26 \\ 1003.44 & 1011.05 & 1015.03 & 1032.06 & 1032.46 & 1036.05 \\ 1041.15 & 1041.76 & 1042.76 & 1054.27 & 1093.38 & 1140.30 \\ 1148.05 & 1149.53 & 1168.28 & 1182.76 & 1183.83 & 1261.56 \\ 1262.73 & 1290.06 & 1321.18 & 1327.90 & 1331.59 & 1341.63 \\ 1357.29 & 1363.36 & 1374.24 & 1383.10 & 1388.98 & 1396.36 \\ 1403.40 & 1438.06 & 1469.03 & 1470.48 & 1472.41 & 1504.10 \\ 1511.06 & 1524.01 & 1555.86 & 1558.36 & 1569.37 & 1578.44 \\ 1602.88 & 1609.65 & 1610.31 & 1623.05 & 1659.39 & 2543.77 \\ 3121.84 & 3124.57 & 3125.25 & 3130.86 & 3154.36 & 3155.16 \\ 3157.79 & 3158.26 & 3173.44 & 3174.15 & 3176.57 & 3177.22\end{array}$

TURBOMOLE Energies and Thermochemistry ${ }^{1}$ RI-PBE/TZVP,SV(P) $-3909.988576$

ZPE

RI-PBE/TZVP,SV(P) + ZPE

RI-PBE/TZVPP

0.329543

B3-LYP/TZVP,SV(P)

$\mathrm{ZPE}(\mathrm{kJ} / \mathrm{mol})$

$-3909.659033$

$-3912.290148$

$-3911.351411$

865.2

$\ln$ (qtrans)

20.24

In(qrot)

17.1

In(qvib)

29.58

chem.pot. $(\mathrm{kJ} / \mathrm{mol})$

699.35

energy $(\mathrm{kJ} / \mathrm{mol})$

951.64

entropy $(\mathrm{kJ} / \mathrm{mol} / \mathrm{K})$

0.8545

$\mathrm{Cv}(\mathrm{kJ} / \mathrm{mol}-\mathrm{K})$

0.5370184

$\mathrm{Cp}((\mathrm{kJ} / \mathrm{mol}-\mathrm{K})$

0.5453327

RI - PBE/TZVP, SV (P) $\quad<\mathrm{S}^{2}>$

0.86

$\mathrm{RI}-\mathrm{PBE} / \mathrm{TZVPP}<\mathrm{S}^{2}>$

0.88

B3 - LYP/TZVP, SV (P) $<\mathrm{S}^{2}>$

1.40

${ }^{1}$ Freeh output; $298.15 \mathrm{~K}, 0.1 \mathrm{MPa}$, unscaled 
${ }^{2} \mathbf{A}$

Chloropentafluorobenzene: $[($ Porphyrin $)(\mathrm{Fe}=$ O)(SH)] $\sigma$-Complex ( $p$-F-addition)

RI-PBE/TZVP, SV(P) Cartesian coordinates

$$
52
$$$$
\text { Energy }=-3910.017614239
$$

\begin{tabular}{|c|c|c|c|}
\hline $\mathrm{C}$ & -0.6705507 & 3.0291111 & 0.7962130 \\
\hline $\mathrm{N}$ & -0.9745162 & 1.7761058 & 0.3046168 \\
\hline $\mathrm{C}$ & -1.6616653 & 1.9899275 & -0.8721256 \\
\hline $\mathrm{C}$ & -1.7820552 & 3.4074051 & -1.1378796 \\
\hline $\mathrm{C}$ & -1.1601792 & 4.0542781 & -0.1006825 \\
\hline $\mathrm{Fe}$ & -0.5360206 & 0.0096556 & 1.1788944 \\
\hline 0 & 1.1951024 & 0.0142063 & 0.4045659 \\
\hline C & -2.1531511 & 0.9875137 & -1.7083574 \\
\hline $\mathrm{C}$ & -1.9921181 & -0.3802976 & -1.4760501 \\
\hline $\mathrm{N}$ & -1.3558354 & -0.9383407 & -0.3859539 \\
\hline $\mathrm{C}$ & -1.4243555 & -2.3110416 & -0.5719422 \\
\hline $\mathrm{C}$ & -2.0946572 & -2.6154441 & -1.8146195 \\
\hline $\mathrm{C}$ & -2.4521927 & -1.4145302 & -2.3755301 \\
\hline $\mathrm{C}$ & -0.9363819 & -3.2776697 & 0.3056497 \\
\hline $\mathrm{C}$ & -0.3197258 & -3.0130454 & 1.5310601 \\
\hline $\mathrm{N}$ & -0.0838165 & -1.7627983 & 2.0526452 \\
\hline $\mathrm{C}$ & 0.5218294 & -1.9666733 & 3.2715325 \\
\hline $\mathrm{C}$ & 0.6701422 & -3.3855652 & 3.5291899 \\
\hline $\mathrm{C}$ & 0.1502509 & -4.0380476 & 2.4410161 \\
\hline $\mathrm{C}$ & 0.9512116 & -0.9625181 & 4.1401413 \\
\hline $\mathrm{C}$ & 0.8503418 & 0.4073130 & 3.8834011 \\
\hline $\mathrm{N}$ & 0.2820809 & 0.9700416 & 2.76289 \\
\hline C & 0.4305594 & 2.3304922 & 2.90 \\
\hline $\mathrm{C}$ & 1.1044509 & 2.6382652 & 4.14831 \\
\hline C & 1.3614946 & 1.4384374 & 4.7624137 \\
\hline C & -0.0031978 & 3.2949860 & 1.9914884 \\
\hline S & -2.5316129 & -0.0027108 & 2.1742745 \\
\hline $\mathrm{H}$ & -2.1059162 & -0.1601520 & 3.4701564 \\
\hline $\mathrm{H}$ & 1.8639974 & 1.2498623 & 5.7205066 \\
\hline $\mathrm{H}$ & 1.3459896 & 3.6526722 & 4.4928358 \\
\hline $\mathrm{H}$ & 0.1792350 & 4.3504511 & 2.2451069 \\
\hline $\mathrm{H}$ & -1.0368960 & 5.1335433 & 0.0607431 \\
\hline $\mathrm{H}$ & -2.2782365 & 3.8364255 & -2.0183997 \\
\hline $\mathrm{H}$ & -2.6812058 & 1.2945927 & -2.6234164 \\
\hline $\mathrm{H}$ & -2.9780918 & -1.2285206 & -3.32 \\
\hline $\mathrm{H}$ & -2.2644113 & -3.6301743 & -2.1 \\
\hline $\mathrm{H}$ & -1.0585994 & -4.3322556 & 0.0158072 \\
\hline $\mathrm{H}$ & 0.08 & -5.1181719 & 2.2 \\
\hline $\mathrm{H}$ & 1.12 & -3.8110475 & 4.4328500 \\
\hline $\mathrm{H}$ & 1.4260375 & -1.2718528 & 5.0835453 \\
\hline $\mathrm{C}$ & 1.8730991 & -0.0210678 & -0.7538957 \\
\hline $\mathrm{C}$ & 1.6362135 & 1.2290522 & -1.5733441 \\
\hline $\mathrm{C}$ & 1.5818214 & -1.2687378 & -1.5621431 \\
\hline $\mathrm{C}$ & 1.0221602 & -1.2557040 & -2.8221826 \\
\hline $\mathrm{C}$ & 1.0375507 & 1.2225000 & -2.8143662 \\
\hline $\mathrm{C}$ & 14294 & -0.0138549 & -3.4582007 \\
\hline $\mathrm{F}$ & 1.93 & 2.3742891 & -0.9780593 \\
\hline $\mathrm{F}$ & 0.73 & 2.3664136 & -3.4374090 \\
\hline $\mathrm{F}$ & 0.74 & -2.3958379 & -3.4 \\
\hline $\mathrm{F}$ & 1.85 & -2.4162908 & -0.9 \\
\hline $\mathrm{F}$ & 3.2 & -0.0591762 & -0.4 \\
\hline $\mathrm{Cl}$ & 0.0 & -0.0015825 & -5.014756 \\
\hline
\end{tabular}

$\begin{array}{rrrrrr}0.00 & 0.00 & 0.00 & 0.00 & 0.00 & 0.00 \\ 9.80 & 26.54 & 39.53 & 49.75 & 54.21 & 61.56 \\ 67.43 & 75.38 & 86.19 & 99.81 & 102.04 & 120.31 \\ 127.52 & 138.23 & 149.82 & 152.09 & 178.79 & 188.43 \\ 199.40 & 206.59 & 215.29 & 233.61 & 249.00 & 252.16 \\ 263.39 & 270.76 & 273.33 & 274.70 & 282.71 & 284.43 \\ 291.73 & 296.85 & 304.17 & 328.73 & 342.32 & 348.22 \\ 356.10 & 358.98 & 365.23 & 385.32 & 390.47 & 399.40 \\ 402.38 & 416.03 & 419.35 & 420.41 & 421.40 & 422.02 \\ 459.03 & 471.87 & 495.10 & 557.99 & 606.91 & 613.15 \\ 650.41 & 655.02 & 655.89 & 659.06 & 673.56 & 683.46 \\ 695.11 & 695.51 & 696.23 & 702.33 & 717.73 & 723.10 \\ 733.80 & 736.55 & 756.97 & 761.26 & 770.75 & 771.06 \\ 778.34 & 782.99 & 784.57 & 785.93 & 798.90 & 832.67 \\ 842.02 & 846.44 & 852.31 & 869.21 & 887.44 & 891.84 \\ 892.65 & 894.36 & 933.01 & 981.52 & 991.17 & 999.90 \\ 1001.62 & 1010.94 & 1013.89 & 1031.69 & 1032.25 & 1036.24 \\ 1040.78 & 1042.14 & 1042.38 & 1053.88 & 1091.65 & 1125.51 \\ 1139.10 & 1144.85 & 1148.15 & 1178.19 & 1181.83 & 1183.00 \\ 1258.96 & 1263.54 & 1305.52 & 1315.69 & 1327.83 & 1328.91 \\ 1332.09 & 1344.28 & 1363.71 & 1373.52 & 1379.93 & 1387.95 \\ 1389.92 & 1394.91 & 1416.93 & 1468.91 & 1470.77 & 1473.81 \\ 1506.43 & 1522.13 & 1555.15 & 1557.39 & 1560.00 & 1579.02 \\ 1604.61 & 1609.56 & 1618.95 & 1624.94 & 1661.06 & 2534.56 \\ 3123.43 & 3125.37 & 3125.58 & 3128.31 & 3154.39 & 3155.06 \\ 3155.84 & 3157.29 & 3173.52 & 3174.07 & 3174.89 & 3176.42\end{array}$

TURBOMOLE Energies and Thermochemistry ${ }^{1}$

\begin{tabular}{|c|c|}
\hline RI-PBE/TZVP,SV(P) & -3910.017614 \\
\hline ZPE & 0.3299749 \\
\hline RI-PBE/TZVP,SV(P) + ZPE & -3909.687639 \\
\hline RI-PBE/TZVPP & -3912.31742 \\
\hline B3-LYP/TZVP,SV(P) & -3911.391982 \\
\hline $\mathrm{ZPE}(\mathrm{kJ} / \mathrm{mol})$ & 866.3 \\
\hline In(qtrans) & 20.24 \\
\hline $\ln$ (qrot) & 17.1 \\
\hline $\ln (q v i b)$ & 30.73 \\
\hline chem.pot. (kJ/mol) & 697.61 \\
\hline energy $(\mathrm{kJ} / \mathrm{mol})$ & 953.78 \\
\hline entropy (kJ/mol/K) & 0.86752 \\
\hline $\mathrm{Cv}(\mathrm{kJ} / \mathrm{mol}-\mathrm{K})$ & 0.5416179 \\
\hline $\mathrm{Cp}((\mathrm{kJ} / \mathrm{mol}-\mathrm{K})$ & 0.5499322 \\
\hline RI - PBE /TZVP , SV (P) & 1.26 \\
\hline $\mathrm{RI}-\mathrm{PBE} / \mathrm{TZVPP}<\mathrm{S}^{2}>$ & 1.26 \\
\hline B3 - LYP / TZVP , SV (P) $\quad\left\langle\mathrm{S}^{2}>\right.$ & 1.70 \\
\hline
\end{tabular}

${ }^{1}$ Freeh output; 298.15K, 0.1MPa, unscaled 
${ }^{4} \mathbf{A}$

Chloropentafluorobenzene: $[($ Porphyrin $)(\mathrm{Fe}=$ O)(SH)] Prereactant Complex ( $p$-F-addition)

RI-PBE/TZVP, SV (P) Cartesian coordinates

52

Energy $=-3909.993928173$

\begin{tabular}{|c|c|c|c|}
\hline & & & \\
\hline C & -2.2383157 & 1.3332272 & 2.6929742 \\
\hline $\mathrm{N}$ & -1.8314184 & 0.2744383 & 1.9183977 \\
\hline C & -2.9706259 & -0.4093062 & 1.5668577 \\
\hline $\mathrm{C}$ & -4.1382454 & 0.2460575 & 2.1224176 \\
\hline C & -3.6787747 & 1.3141129 & 2.8515098 \\
\hline $\mathrm{Fe}$ & 0.0361691 & -0.1596290 & 1.3291610 \\
\hline $\mathrm{O}$ & -0.2223218 & 0.4503426 & -0.2020045 \\
\hline $\mathrm{C}$ & -2.9981573 & -1.6367758 & 0.8942863 \\
\hline C & -1.8681288 & -2.3972104 & 0.5814169 \\
\hline $\mathrm{N}$ & -0.5700241 & -1.9938106 & 0.7989191 \\
\hline $\mathrm{C}$ & 0.2324180 & -3.0619092 & 0.4616522 \\
\hline C & -0.5850570 & -4.1823935 & 0.0448555 \\
\hline C & -1.8900470 & -3.7606687 & 0.0937417 \\
\hline C & 1.6285056 & -3.0307592 & 0.3983025 \\
\hline C & 2.4143865 & -1.8899255 & 0.5755576 \\
\hline $\mathrm{N}$ & 1.9390640 & -0.6419159 & 0.929512 \\
\hline $\mathrm{C}$ & 3.0036561 & 0.2255136 & 0.8174380 \\
\hline $\mathrm{C}$ & 4.1866496 & -0.4911988 & 0.3905855 \\
\hline C & 3.8260951 & -1.8096500 & 0.2662833 \\
\hline C & 2.9623800 & 1.5777899 & 1.1671412 \\
\hline C & 1.8759781 & 2.2032247 & 1.7896241 \\
\hline $\mathrm{N}$ & 0.6611707 & 1.6080984 & 2.0414197 \\
\hline C & -0.0594124 & 2.5045144 & 2.7961404 \\
\hline C & 0.7288898 & 3.6934035 & 3.0481760 \\
\hline $\mathrm{C}$ & 1.9224939 & 3.5194084 & 2.3927828 \\
\hline C & -1.4002107 & 2.3496489 & 3.1624471 \\
\hline S & 0.5092562 & -1.2363116 & 3.4602505 \\
\hline $\mathrm{H}$ & 1.5943528 & -0.4779864 & 3.819563 \\
\hline $\mathrm{H}$ & 2.7804612 & 4.2024564 & 2.3373214 \\
\hline $\mathrm{H}$ & 0.3866822 & 4.5587250 & 3.631242 \\
\hline $\mathrm{H}$ & -1.8571662 & 3.1475964 & 3.7677935 \\
\hline $\mathrm{H}$ & -4.2548524 & 2.0487269 & 3.4298127 \\
\hline $\mathrm{H}$ & -5.1722107 & -0.0983809 & 1.9875862 \\
\hline $\mathrm{H}$ & -3.9838775 & -2.0789854 & 0.6835848 \\
\hline $\mathrm{H}$ & -2.8008426 & -4.3206787 & -0.1562818 \\
\hline $\mathrm{H}$ & -0.1920068 & -5.1576444 & -0.2714619 \\
\hline $\mathrm{H}$ & 2.1407385 & -3.9531018 & 0.0850688 \\
\hline $\mathrm{H}$ & 4.4462097 & -2.6626170 & -0.0396957 \\
\hline $\mathrm{H}$ & 5.1703996 & -0.0318065 & 0.2265020 \\
\hline $\mathrm{H}$ & 3.8828085 & 2.1671362 & 1.0362278 \\
\hline C & 0.6628480 & -0.9649445 & -2.7894497 \\
\hline C & 1.3762575 & 0.2420080 & -2.829458 \\
\hline C & -0.7289078 & -0.9660596 & -2.9650178 \\
\hline C & -1.4042262 & 0.2386788 & -3.2127487 \\
\hline C & 0.7009875 & 1.4466166 & -3.0766721 \\
\hline C & -0.6918542 & 1.4499579 & -3.2707609 \\
\hline $\mathrm{F}$ & 2.6952030 & 0.2428427 & -2.6103386 \\
\hline $\mathrm{F}$ & 1.3936938 & 2.5858359 & -3.1048082 \\
\hline $\mathrm{F}$ & -2.7280019 & 0.2218891 & -3.3758243 \\
\hline$F$ & -1.4089563 & -2.1141503 & -2.8785802 \\
\hline $\mathrm{Cl}$ & -1.524378 & 2.9329933 & -3.565299 \\
\hline $\mathrm{F}$ & 1.30422 & -2.1101997 & -2.541002 \\
\hline
\end{tabular}

$\begin{array}{rrrrrr}i 10.95 & 0.00 & 0.00 & 0.00 & 0.00 & 0.00 \\ 0.00 & 11.75 & 14.91 & 24.05 & 25.53 & 50.26 \\ 55.90 & 59.97 & 77.11 & 89.49 & 101.80 & 110.37 \\ 130.48 & 137.42 & 142.40 & 150.97 & 165.94 & 174.09 \\ 180.42 & 192.08 & 210.65 & 212.97 & 221.54 & 235.54 \\ 259.88 & 261.24 & 267.15 & 271.10 & 273.51 & 280.37 \\ 287.08 & 297.74 & 298.97 & 311.57 & 319.98 & 345.20 \\ 353.04 & 360.05 & 361.04 & 371.37 & 378.19 & 385.47 \\ 404.33 & 407.16 & 420.62 & 422.35 & 423.05 & 424.34 \\ 428.70 & 458.56 & 485.61 & 509.78 & 531.87 & 569.86 \\ 608.73 & 620.40 & 650.37 & 652.84 & 653.37 & 666.76 \\ 673.52 & 691.79 & 692.86 & 697.65 & 713.42 & 723.81 \\ 733.67 & 734.53 & 750.84 & 763.11 & 769.46 & 769.81 \\ 774.96 & 778.23 & 785.70 & 786.46 & 799.99 & 804.46 \\ 844.63 & 853.20 & 853.63 & 862.00 & 872.04 & 887.20 \\ 892.28 & 893.14 & 895.79 & 995.49 & 995.59 & 1004.95 \\ 1005.51 & 1010.96 & 1017.64 & 1031.05 & 1033.43 & 1036.60 \\ 1040.31 & 1041.28 & 1043.56 & 1055.68 & 1103.23 & 1137.94 \\ 1147.15 & 1148.06 & 1171.45 & 1181.49 & 1185.16 & 1261.76 \\ 1262.53 & 1288.72 & 1292.57 & 1325.55 & 1326.18 & 1338.65 \\ 1358.18 & 1362.70 & 1371.42 & 1379.71 & 1385.09 & 1395.12 \\ 1445.27 & 1463.22 & 1466.41 & 1466.94 & 1498.99 & 1514.93 \\ 1520.21 & 1523.34 & 1551.81 & 1551.89 & 1571.18 & 1578.76 \\ 1599.50 & 1600.68 & 1636.45 & 1637.00 & 1651.84 & 2551.66 \\ 3124.56 & 3126.39 & 3126.46 & 3127.27 & 3156.40 & 3156.86 \\ 3157.66 & 3157.93 & 3175.12 & 3175.54 & 3176.22 & 3176.52\end{array}$

TURBOMOLE Energies and Thermochemistry ${ }^{1}$ RI-PBE/TZVP,SV(P) $-3909.993784$

ZPE

0.3293788

RI-PBE/TZVP,SV(P) + ZPE

RI-PBE/TZVPP

$-3909.664465$

B3-LYP/TZVP,SV(P)

$-3912.300308$

ZPE $(\mathrm{kJ} / \mathrm{mol})$

$-3911.374965$

864.3

$\ln$ (qtrans)

20.24

In(qrot)

17.22

In(qvib)

32.17

chem.pot. $(\mathrm{kJ} / \mathrm{mol})$

691.67

energy $(\mathrm{kJ} / \mathrm{mol})$

951.72

entropy $(\mathrm{kJ} / \mathrm{mol} / \mathrm{K})$

0.88054

$\mathrm{Cv}(\mathrm{kJ} / \mathrm{mol}-\mathrm{K})$

0.5383689

$\mathrm{Cp}((\mathrm{kJ} / \mathrm{mol}-\mathrm{K})$

0.5466832

$\mathrm{RI}$ - PBE/TZVP, SV (P) $<\mathrm{S}^{2}>$

3.77

$\mathrm{RI}-\mathrm{PBE} / \mathrm{TZVPP}<\mathrm{S}^{2}>$

3.77

B3 - LYP/TZVP, SV (P) $\left\langle\mathrm{S}^{2}>\right.$

3.80

${ }^{1}$ Freeh output; $298.15 \mathrm{~K}, 0.1 \mathrm{MPa}$, unscaled 
${ }^{4} \mathbf{A}$

\section{Chloropentafluorobenzene: $[($ Porphyrin $)(\mathrm{Fe}=$ O)(SH)] Transition State Complex ( $p-\mathrm{F}-$ addition)}

RI-PBE/TZVP, SV (P) Cartesian coordinates

$$
52
$$

Energy $=-3909.978617497$

\begin{tabular}{|c|c|c|c|}
\hline $\mathrm{C}$ & -3.0817030 & -1.2172840 & 1.0772660 \\
\hline $\mathrm{N}$ & -1.7244440 & -1.4131570 & 1.1436310 \\
\hline $\mathrm{C}$ & -1.5208860 & -2.7621530 & 0.9755080 \\
\hline $\mathrm{C}$ & -2.7881560 & -3.4410540 & 0.7878400 \\
\hline $\mathrm{C}$ & -3.7634290 & -2.4806790 & 0.8765080 \\
\hline $\mathrm{Fe}$ & -0.3079600 & -0.0047060 & 1.3473680 \\
\hline $\mathrm{O}$ & -0.0879400 & -0.0245790 & -0.3011180 \\
\hline $\mathrm{C}$ & -0.2869220 & -3.4104520 & 1.0921060 \\
\hline $\mathrm{C}$ & 0.9036550 & -2.7932950 & 1.4854690 \\
\hline $\mathrm{N}$ & 1.0505310 & -1.4449190 & 1.7124400 \\
\hline C & 2.3403040 & -1.2686400 & 2.1656210 \\
\hline $\mathrm{C}$ & 3.0178640 & -2.5457760 & 2.2497840 \\
\hline $\mathrm{C}$ & 2.1307140 & -3.4929460 & 1.8044210 \\
\hline $\mathrm{C}$ & 2.9489260 & -0.0340110 & 2.4065270 \\
\hline $\mathrm{C}$ & 2.3666870 & 1.2114110 & 2.1616860 \\
\hline $\mathrm{N}$ & 1.0786590 & 1.4135330 & 1.7069070 \\
\hline $\mathrm{C}$ & 0.9608290 & 2.7655630 & 1.4777360 \\
\hline $\mathrm{C}$ & 2.2020280 & 3.4395610 & 1.7958340 \\
\hline $\mathrm{C}$ & 3.0692090 & 2.4746910 & 2.2431680 \\
\hline $\mathrm{C}$ & -0.2182380 & 3.4043840 & 1.0858120 \\
\hline $\mathrm{C}$ & -1.4655280 & 2.7794900 & 0.9825660 \\
\hline $\mathrm{N}$ & -1.6931980 & 1.4333900 & 1.1574550 \\
\hline $\mathrm{C}$ & -3.0561090 & 1.2622210 & 1.0884610 \\
\hline $\mathrm{C}$ & -3.7128000 & 2.5377820 & 0.8875220 \\
\hline C & -2.7196900 & 3.4801470 & 0.7957960 \\
\hline $\mathrm{C}$ & -3.7148090 & 0.0289580 & 1.1046700 \\
\hline S & -0.5987610 & -0.0183770 & 3.6979180 \\
\hline $\mathrm{H}$ & -0.8252690 & 1.3228790 & 3.8753950 \\
\hline $\mathrm{H}$ & -2.8136070 & 4.5626780 & 0.6368750 \\
\hline $\mathrm{H}$ & -4.7991180 & 2.6756930 & 0.8046460 \\
\hline $\mathrm{H}$ & -4.8137650 & 0.0411470 & 1.0384140 \\
\hline $\mathrm{H}$ & -4.8522260 & -2.5982610 & 0.7950330 \\
\hline $\mathrm{H}$ & -2.9016670 & -4.5223740 & 0.6336310 \\
\hline $\mathrm{H}$ & -0.2703280 & -4.5015100 & 0.9467570 \\
\hline $\mathrm{H}$ & 2.2739750 & -4.5777490 & 1.7121990 \\
\hline $\mathrm{H}$ & 4.0531760 & -2.6820450 & 2.5895470 \\
\hline $\mathrm{H}$ & 3.9948980 & -0.0443360 & 2.7490910 \\
\hline $\mathrm{H}$ & 4.1071300 & 2.5904230 & 2.5826200 \\
\hline $\mathrm{H}$ & 2.3684400 & 4.5208720 & 1.7011180 \\
\hline $\mathrm{H}$ & -0.1811350 & 4.4940230 & 0.9337060 \\
\hline $\mathrm{C}$ & 1.5405660 & 0.0024370 & -1.6618850 \\
\hline C & 1.1898970 & 1.2227250 & -2.2701550 \\
\hline $\mathrm{C}$ & 1.2056380 & -1.2139900 & -2.2869390 \\
\hline $\mathrm{C}$ & 0.4308510 & -1.2100640 & -3.4515130 \\
\hline $\mathrm{C}$ & 0.4137290 & 1.2247030 & -3.4344330 \\
\hline $\mathrm{C}$ & 0.0638900 & 0.0094310 & -4.0545740 \\
\hline $\mathrm{F}$ & 1.5341990 & 2.3781830 & -1.6875640 \\
\hline $\mathrm{F}$ & 0.0750180 & 2.3893110 & -3.9946100 \\
\hline $\mathrm{F}$ & 0.1081080 & -2.3706160 & -4.0289870 \\
\hline $\mathrm{F}$ & 1.56 & -2.3715920 & -1.7168790 \\
\hline $\mathrm{Cl}$ & -0.84 & 0.0131640 & -5.5200340 \\
\hline $\mathrm{F}$ & 2.45 & 0.0002400 & -0.6824200 \\
\hline
\end{tabular}

$\begin{array}{rrrrrr}1487.70 & 0.00 & 0.00 & 0.00 & 0.00 & 0.00 \\ 0.00 & 14.79 & 25.31 & 28.23 & 50.22 & 53.62 \\ 58.95 & 60.07 & 76.14 & 98.91 & 101.92 & 112.12 \\ 130.90 & 137.84 & 143.75 & 149.77 & 166.39 & 171.94 \\ 192.73 & 202.56 & 211.93 & 237.83 & 238.78 & 245.27 \\ 254.17 & 259.75 & 263.76 & 273.50 & 284.77 & 286.92 \\ 291.92 & 296.76 & 297.77 & 298.49 & 321.49 & 346.69 \\ 351.03 & 360.17 & 363.66 & 366.83 & 384.83 & 399.92 \\ 411.29 & 415.39 & 420.86 & 421.39 & 422.52 & 422.58 \\ 427.28 & 458.81 & 484.73 & 496.32 & 572.78 & 612.07 \\ 618.74 & 642.83 & 651.83 & 655.29 & 656.18 & 670.64 \\ 674.50 & 693.80 & 694.73 & 697.80 & 715.93 & 721.81 \\ 724.37 & 734.15 & 735.32 & 752.65 & 763.94 & 770.61 \\ 770.98 & 776.32 & 780.02 & 785.96 & 786.30 & 800.30 \\ 843.38 & 851.96 & 852.49 & 860.57 & 874.80 & 886.69 \\ 892.13 & 892.32 & 894.42 & 991.16 & 995.53 & 1004.76 \\ 1005.50 & 1011.06 & 1017.37 & 1032.16 & 1032.48 & 1035.47 \\ 1040.51 & 1041.63 & 1042.59 & 1056.02 & 1086.61 & 1138.46 \\ 1147.16 & 1148.58 & 1160.48 & 1181.22 & 1184.18 & 1261.22 \\ 1263.17 & 1284.34 & 1301.81 & 1327.19 & 1328.07 & 1338.39 \\ 1352.44 & 1363.49 & 1368.62 & 1381.64 & 1385.12 & 1387.17 \\ 1390.35 & 1431.42 & 1465.47 & 1467.53 & 1468.76 & 1501.14 \\ 1510.57 & 1521.42 & 1553.20 & 1554.06 & 1561.66 & 1573.85 \\ 1585.52 & 1601.94 & 1603.77 & 1618.27 & 1654.88 & 2550.20 \\ 3123.11 & 3125.52 & 3125.64 & 3125.94 & 3155.20 & 3155.37 \\ 3155.71 & 3155.93 & 3173.96 & 3174.20 & 3174.51 & 3174.74\end{array}$

TURBOMOLE Energies and Thermochemistry ${ }^{1}$

\begin{tabular}{|c|c|}
\hline RI-PBE/TZVP,SV(P) & -3909.978617 \\
\hline ZPE & 0.3290395 \\
\hline RI-PBE/TZVP,SV(P) + ZPE & -3909.649578 \\
\hline RI-PBE/TZVPP & -3912.28134 \\
\hline B3-LYP/TZVP,SV(P) & $\mathrm{NC}$ \\
\hline $\mathrm{ZPE}(\mathrm{kJ} / \mathrm{mol})$ & 863.9 \\
\hline In(qtrans) & 20.24 \\
\hline In(qrot) & 17.25 \\
\hline $\ln (q v i b)$ & 30.24 \\
\hline chem.pot. (kJ/mol) & 695.99 \\
\hline energy (kJ/mol) & 950.54 \\
\hline entropy $(\mathrm{kJ} / \mathrm{mol} / \mathrm{K})$ & 0.86208 \\
\hline $\mathrm{Cv}(\mathrm{kJ} / \mathrm{mol}-\mathrm{K})$ & 0.537414 \\
\hline $\mathrm{Cp}((\mathrm{kJ} / \mathrm{mol}-\mathrm{K})$ & 0.5457283 \\
\hline $\mathrm{RI}-\mathrm{PBE} / \mathrm{TZVP}, \mathrm{SV}(\mathrm{P}) \quad<\mathrm{S}^{2}>$ & 3.77 \\
\hline $\mathrm{RI}-\mathrm{PBE} / \mathrm{TZVPP}<\mathrm{S}^{2}>$ & 3.77 \\
\hline B3 - LYP / TZVP , SV (P) $\quad\left\langle\mathrm{S}^{2}>\right.$ & 99.57 \\
\hline
\end{tabular}

${ }^{1}$ Freeh output; $298.15 \mathrm{~K}, 0.1 \mathrm{MPa}$, unscaled 
Chloropentafluorobenzene: $[($ Porphyrin $)(\mathrm{Fe}=$ O)(SH)] $\sigma$-Complex Complex ( $p$-F-addition)

RI-PBE/TZVP, SV(P) Cartesian coordinates

$$
52
$$$$
\text { Energy }=-3910.013145921
$$

\begin{tabular}{|c|c|c|c|}
\hline $\mathrm{C}$ & -2.9600925 & -1.2248945 & 1.3474330 \\
\hline $\mathrm{N}$ & -1.5975046 & -1.4260703 & 1.3774370 \\
\hline C & -1.4089525 & -2.7876191 & 1.3091708 \\
\hline $\mathrm{C}$ & -2.6847125 & -3.4670919 & 1.2269768 \\
\hline $\mathrm{C}$ & -3.6515514 & -2.4937263 & 1.2605869 \\
\hline $\mathrm{Fe}$ & -0.1603276 & 0.0021364 & 1.4754754 \\
\hline 0 & -0.1391327 & -0.0038651 & -0.4203552 \\
\hline C & -0.1747665 & -3.4369384 & 1.3553220 \\
\hline $\mathrm{C}$ & 1.0514708 & -2.7936826 & 1.5253551 \\
\hline $\mathrm{N}$ & 1.2406174 & -1.4310711 & 1.6150325 \\
\hline C & 2.5903622 & -1.2447053 & 1.8321150 \\
\hline C & 3.2692224 & -2.5195259 & 1.8794640 \\
\hline C & 2.3130664 & -3.4836776 & 1.6777025 \\
\hline C & 3.2318336 & -0.0125596 & 1.9530024 \\
\hline C & 2.6047159 & 1.2258252 & 1.8171180 \\
\hline $\mathrm{N}$ & 1.2575813 & 1.4238456 & 1.6001409 \\
\hline C & 1.0809268 & 2.7874612 & 1.5030363 \\
\hline C & 2.3498444 & 3.4666685 & 1.6474377 \\
\hline C & 3.2966964 & 2.4944402 & 1.8536388 \\
\hline C & -0.1419687 & 3.4393939 & 1.3402811 \\
\hline C & -1.3824314 & 2.8000004 & 1.3064534 \\
\hline $\mathrm{N}$ & -1.5830272 & 1.4390521 & 1.3845303 \\
\hline C & -2.9479301 & 1.2490783 & 1.3506797 \\
\hline C & -3.6273526 & 2.5229895 & 1.2586607 \\
\hline C & -2.6519551 & 3.4887594 & 1.2218385 \\
\hline C & -3.6000793 & 0.0148145 & 1.3576985 \\
\hline S & -0.3369222 & -0.0342173 & 3.7226123 \\
\hline $\mathrm{H}$ & -0.4405995 & 1.3207589 & 3.9146133 \\
\hline $\mathrm{H}$ & -2.7662966 & 4.5784664 & 1.1463627 \\
\hline $\mathrm{H}$ & -4.7178586 & 2.6461526 & 1.2169129 \\
\hline $\mathrm{H}$ & -4.7001355 & 0.0206064 & 1.3279316 \\
\hline $\mathrm{H}$ & -4.7432162 & -2.6075197 & 1.22228992 \\
\hline $\mathrm{H}$ & -2.8076651 & -4.5563417 & 1.1592593 \\
\hline $\mathrm{H}$ & -0.1709810 & -4.5354656 & 1.2933705 \\
\hline $\mathrm{H}$ & 2.4344675 & -4.5744229 & 1.6376075 \\
\hline $\mathrm{H}$ & 4.3483897 & -2.6448536 & 2.0397593 \\
\hline $\mathrm{H}$ & 4.3190783 & -0.0180331 & 2.1218291 \\
\hline $\mathrm{H}$ & 4.3775451 & 2.6099675 & 2.0098432 \\
\hline $\mathrm{H}$ & 2.4819845 & 4.5558784 & 1.5996632 \\
\hline $\mathrm{H}$ & -0.1303434 & 4.5374772 & 1.2708247 \\
\hline C & 0.7551317 & 0.0009274 & -1.4384588 \\
\hline C & 0.5795708 & 1.2453753 & -2.2807261 \\
\hline C & 0.5897637 & -1.2474210 & -2.2774054 \\
\hline $\mathrm{C}$ & 0.1733316 & -1.2386021 & -3.5905484 \\
\hline $\mathrm{C}$ & 0.1586464 & 1.2290774 & -3.5925620 \\
\hline C & -0.0287598 & -0.0064059 & -4.2786347 \\
\hline $\mathrm{F}$ & 81041 & 2.4041367 & -1.6443925 \\
\hline $\mathrm{F}$ & -0.0480762 & 2.3761116 & -4.2498859 \\
\hline $\mathrm{F}$ & -0.0237709 & -2.3891394 & -4.2445710 \\
\hline $\mathrm{F}$ & 0.7669634 & -2.4023415 & -1.636813 \\
\hline $\mathrm{Cl}$ & -0.5240747 & -0.0107134 & -5.9205789 \\
\hline $\mathrm{F}$ & 2.0971586 & 0.0059661 & -0.9582040 \\
\hline
\end{tabular}

$\begin{array}{rrrrrr}0.00 & 0.00 & 0.00 & 0.00 & 0.00 & 0.00 \\ 11.11 & 18.02 & 33.39 & 38.21 & 45.08 & 60.35 \\ 68.11 & 72.56 & 77.16 & 103.44 & 104.76 & 117.57 \\ 129.07 & 135.01 & 147.88 & 148.65 & 150.78 & 194.81 \\ 199.85 & 208.19 & 208.81 & 233.37 & 252.67 & 253.71 \\ 260.18 & 264.05 & 273.96 & 275.86 & 277.80 & 289.75 \\ 291.90 & 297.56 & 312.45 & 319.12 & 326.81 & 354.25 \\ 357.33 & 359.66 & 365.64 & 393.02 & 399.21 & 400.05 \\ 415.61 & 418.00 & 420.22 & 420.90 & 422.19 & 423.28 \\ 457.99 & 468.99 & 483.36 & 549.39 & 594.05 & 599.22 \\ 648.11 & 648.57 & 655.63 & 659.54 & 671.45 & 674.58 \\ 685.28 & 695.44 & 696.21 & 697.09 & 720.18 & 723.42 \\ 733.69 & 734.91 & 759.21 & 761.56 & 770.98 & 772.18 \\ 779.53 & 782.76 & 783.44 & 786.74 & 798.63 & 833.38 \\ 842.52 & 843.27 & 850.58 & 856.37 & 888.12 & 892.88 \\ 893.03 & 894.61 & 909.84 & 980.67 & 994.36 & 1001.15 \\ 1002.67 & 1014.56 & 1017.38 & 1033.23 & 1033.92 & 1037.75 \\ 1043.22 & 1043.99 & 1044.32 & 1056.24 & 1087.48 & 1121.84 \\ 1139.00 & 1143.83 & 1144.55 & 1178.03 & 1184.87 & 1197.16 \\ 1259.56 & 1261.49 & 1287.27 & 1305.16 & 1330.10 & 1331.26 \\ 1333.19 & 1347.72 & 1365.88 & 1377.80 & 1381.18 & 1384.39 \\ 1384.95 & 1386.67 & 1402.92 & 1471.30 & 1471.77 & 1473.25 \\ 1510.39 & 1520.59 & 1554.29 & 1557.03 & 1559.46 & 1579.37 \\ 1603.48 & 1606.85 & 1620.90 & 1621.17 & 1661.98 & 2544.44 \\ 3125.36 & 3127.38 & 3127.65 & 3128.13 & 3154.92 & 3155.13 \\ 3155.54 & 3155.79 & 3173.71 & 3174.00 & 3174.35 & 3174.59\end{array}$

TURBOMOLE Energies and Thermochemistry ${ }^{1}$

\begin{tabular}{|c|c|}
\hline RI-PBE/TZVP,SV(P) & -3910.013146 \\
\hline ZPE & 0.3295842 \\
\hline RI-PBE/TZVP,SV(P) + ZPE & -3909.683562 \\
\hline RI-PBE/TZVPP & -3912.314409 \\
\hline B3-LYP/TZVP,SV(P) & -3911.396784 \\
\hline $\mathrm{ZPE}(\mathrm{kJ} / \mathrm{mol})$ & 865.3 \\
\hline In(qtrans) & 20.24 \\
\hline In(qrot) & 17.28 \\
\hline $\ln (q v i b)$ & 31.78 \\
\hline chem.pot. (kJ/mol) & 693.55 \\
\hline energy $(\mathrm{kJ} / \mathrm{mol})$ & 953.22 \\
\hline entropy $(\mathrm{kJ} / \mathrm{mol} / \mathrm{K})$ & 0.87925 \\
\hline $\mathrm{Cv}(\mathrm{kJ} / \mathrm{mol}-\mathrm{K})$ & 0.5424947 \\
\hline $\mathrm{Cp}((\mathrm{kJ} / \mathrm{mol}-\mathrm{K})$ & 0.550809 \\
\hline $\mathrm{RI}-\mathrm{PBE} / \mathrm{TZVP}, \mathrm{SV}(\mathrm{P}) \quad<\mathrm{S}^{2}>$ & 3.79 \\
\hline $\mathrm{RI}-\mathrm{PBE} / \mathrm{TZVPP}<\mathrm{S}^{2}>$ & 3.79 \\
\hline B3 - LYP/TZVP, SV (P) $\left\langle\mathrm{S}^{2}>\right.$ & 3.87 \\
\hline
\end{tabular}

${ }^{1}$ Freeh output; $298.15 \mathrm{~K}, 0.1 \mathrm{MPa}$, unscaled 


\section{Benzene}

$$
\begin{aligned}
& 12 \\
& \text { Energy }=-231.7608083998
\end{aligned}
$$

$\begin{array}{lrrr}\mathrm{C} & -1.2167435 & 0.7024872 & 0.0000000 \\ \mathrm{C} & -1.2167435 & -0.7024872 & 0.0000000 \\ \mathrm{C} & 0.0000000 & -1.4049744 & 0.0000000 \\ \mathrm{C} & 1.2167435 & -0.7024872 & 0.0000000 \\ \mathrm{C} & 1.2167435 & 0.7024872 & 0.0000000 \\ \mathrm{C} & 0.0000000 & 1.4049744 & 0.0000000 \\ \mathrm{H} & -2.1718830 & 1.2539372 & 0.0000000 \\ \mathrm{H} & -2.1718830 & -1.2539372 & 0.0000000 \\ \mathrm{H} & 0.0000000 & -2.5078744 & 0.0000000 \\ \mathrm{H} & 2.1718830 & -1.2539372 & 0.0000000 \\ \mathrm{H} & 2.1718830 & 1.2539372 & 0.0000000 \\ \mathrm{H} & 0.0000000 & 2.5078744 & 0.0000000\end{array}$

Vibrational frequencies

$\begin{array}{rrrrrr}0.00 & 0.00 & 0.00 & 0.00 & 0.00 & 0.00 \\ 401.35 & 401.35 & 599.15 & 599.15 & 669.32 & 714.74 \\ 838.05 & 838.05 & 956.42 & 956.42 & 978.57 & 994.41 \\ 1002.84 & 1038.05 & 1038.05 & 1132.70 & 1156.19 & 1156.19 \\ 1329.72 & 1381.46 & 1474.54 & 1474.54 & 1617.01 & 1617.01 \\ 3088.39 & 3098.69 & 3098.69 & 3113.38 & 3113.38 & 3122.91\end{array}$

TURBOMOLE Energies and Thermochemistry ${ }^{1}$

\begin{tabular}{lr}
\hline RI-PBE/TZVP,SV(P) & -231.7608084 \\
\hline ZPE & 0.0979629 \\
RI-PBE/TZVP,SV(P) + ZPE & -231.6628455 \\
RI-PBE/TZVPP & -232.0213854 \\
B3-LYP/TZVP,SV(P) & -231.9160297 \\
ZPE (kJ/mol) & 257.2 \\
In(qtrans) & 17.14 \\
In(qrot) & 8.96 \\
In (qvib) & 0.61 \\
chem.pot. (kJ/mol) & 190.98 \\
energy $(\mathrm{kJ} / \mathrm{mol})$ & 269.01 \\
entropy $(\mathrm{kJ} / \mathrm{mol} / \mathrm{K})$ & 0.27002 \\
Cv $(\mathrm{kJ} / \mathrm{mol}-\mathrm{K})$ & 0.0742898 \\
$\mathrm{Cp}((\mathrm{kJ} / \mathrm{mol}-\mathrm{K})$ & 0.0826041 \\
\hline
\end{tabular}

${ }^{1}$ Freeh output; $298.15 \mathrm{~K}, 0.1 \mathrm{MPa}$, unscaled 
${ }^{2}$ A Benzene:[(Porphyrin $\left.)(\mathrm{Fe}=\mathbf{O})(\mathrm{SH})\right]$ Prereactant Complex

RI-PBE/TZVP, SV (P) Cartesian coordinates

52

Energy $=-2955.367287192$

\begin{tabular}{|c|c|c|c|}
\hline $\mathrm{C}$ & -2.7249581 & 1.9924616 & -0.4084944 \\
\hline $\mathrm{N}$ & -2.0111124 & 0.9629817 & 0.1660361 \\
\hline C & -2.9406967 & 0.0663489 & 0.6324323 \\
\hline $\mathrm{C}$ & -4.2819549 & 0.5421044 & 0.3641076 \\
\hline C & -4.1468630 & 1.7421940 & -0.2861038 \\
\hline $\mathrm{Fe}$ & 0.0161381 & 0.7411157 & 0.1789893 \\
\hline 0 & -0.0150102 & 0.1583208 & -1.3543932 \\
\hline C & -2.6380737 & -1.1392353 & 1.2707271 \\
\hline C & -1.3487635 & -1.6104368 & 1.5189436 \\
\hline $\mathrm{N}$ & -0.1749656 & -0.9732111 & 699608 \\
\hline C & 0.8436926 & -1.7798431 & 1.6344474 \\
\hline C & 0.3020299 & -2.9541985 & 2.2831365 \\
\hline C & -1.0621201 & -2.8496912 & 2.2095994 \\
\hline C & 2.2070721 & -1.5141056 & 1.5115413 \\
\hline $\mathrm{C}$ & 2.7480761 & -0.3810053 & 0.8994149 \\
\hline $\mathrm{N}$ & 2.0181537 & 0.6401262 & 0.3359071 \\
\hline C & 2.9405471 & 1.5341003 & -0.1680812 \\
\hline C & 4.2856321 & 1.0674243 & 0.0959941 \\
\hline C & 4.1659259 & -0.1252331 & 0.7631894 \\
\hline C & 2.6513262 & 2.7281004 & -0.8345058 \\
\hline $\mathrm{C}$ & 1.3700342 & 3.2182428 & -1.0940142 \\
\hline $\mathrm{N}$ & 0.2019439 & 2.5982352 & -0.7185448 \\
\hline C & -0.8245448 & 3.3923300 & -1.1791675 \\
\hline C & -0.2875654 & 4.5622757 & -1.845163 \\
\hline C & 1.0790722 & 4.4546798 & -1.7918866 \\
\hline C & -2.1852110 & 3.1188184 & -1.0340647 \\
\hline S & -0.1313792 & 2.0997403 & 2.0861176 \\
\hline $\mathrm{H}$ & 1.1560783 & 2.5755251 & 2.0925990 \\
\hline $\mathrm{H}$ & 1.8379226 & 5.1427506 & -2.1876470 \\
\hline $\mathrm{H}$ & -0.8959483 & 5.3585946 & -2.2944629 \\
\hline $\mathrm{H}$ & -2.8942243 & 3.8495755 & -1.4522402 \\
\hline $\mathrm{H}$ & -4.9315868 & 2.4136615 & -0.6591317 \\
\hline $\mathrm{H}$ & -5.2016931 & 0.0108863 & 0.6430501 \\
\hline $\mathrm{H}$ & -3.4795486 & -1.7647269 & 1.6054654 \\
\hline $\mathrm{H}$ & -1.8259365 & -3.5434613 & 2.5844647 \\
\hline $\mathrm{H}$ & 0.9074133 & -3.7539079 & 2.729588 \\
\hline $\mathrm{H}$ & 2.9083320 & -2.2527359 & 1.928236 \\
\hline $\mathrm{H}$ & 4.9582858 & -0.7878662 & 1.1357002 \\
\hline $\mathrm{H}$ & 5.1989174 & 1.6000409 & -0.201013 \\
\hline $\mathrm{H}$ & 3.5050605 & 3.3282332 & -1.1847023 \\
\hline C & -0.0807320 & -3.2254815 & -1.6872201 \\
\hline C & 1.1726002 & -3.8588193 & -1.6259424 \\
\hline C & -1.2532257 & -4.0006602 & -1.6990195 \\
\hline C & -1.1739508 & -5.4031065 & -1.6490264 \\
\hline C & 1.2540104 & -5.2610278 & -1.5758612 \\
\hline C & 0.0802465 & -6.0338071 & -1.5870168 \\
\hline $\mathrm{H}$ & 2.0917438 & -3.2492363 & -1.6169874 \\
\hline $\mathrm{H}$ & 2.23875 & -5.7566097 & -1.5303319 \\
\hline $\mathrm{H}$ & 0.14378 & -7.1344017 & -1.548820 \\
\hline $\mathrm{H}$ & -2.09528 & -6.009 & -1.660589 \\
\hline $\mathrm{H}$ & -2.23675 & -3.5035588 & -1.747808 \\
\hline
\end{tabular}

$\begin{array}{rrrrrr}0.00 & 0.00 & 0.00 & 0.00 & 0.00 & 0.00 \\ 4.25 & 11.71 & 17.95 & 25.07 & 29.98 & 39.58 \\ 51.02 & 71.24 & 92.23 & 96.83 & 102.07 & 106.80 \\ 145.45 & 148.10 & 177.77 & 196.44 & 206.25 & 230.34 \\ 236.71 & 270.94 & 273.04 & 274.95 & 278.00 & 286.69 \\ 293.19 & 309.58 & 346.03 & 358.45 & 358.91 & 364.30 \\ 396.18 & 401.28 & 401.37 & 405.94 & 417.59 & 419.13 \\ 419.98 & 422.02 & 459.10 & 593.06 & 598.32 & 601.50 \\ 650.13 & 656.42 & 656.81 & 672.45 & 675.23 & 678.95 \\ 694.28 & 695.00 & 697.53 & 714.45 & 715.47 & 723.86 \\ 734.00 & 735.42 & 764.28 & 772.26 & 772.94 & 778.94 \\ 781.81 & 784.36 & 784.90 & 798.64 & 837.40 & 841.31 \\ 843.30 & 847.16 & 850.46 & 851.04 & 859.09 & 888.00 \\ 892.85 & 893.58 & 896.21 & 955.25 & 963.39 & 981.23 \\ 993.33 & 1000.73 & 1001.57 & 1002.04 & 1002.70 & 1011.44 \\ 1015.04 & 1032.03 & 1033.72 & 1036.70 & 1037.14 & 1038.22 \\ 1041.61 & 1042.23 & 1044.49 & 1054.61 & 1132.54 & 1139.03 \\ 1147.42 & 1148.15 & 1152.60 & 1161.29 & 1182.07 & 1184.01 \\ 1261.11 & 1262.84 & 1312.90 & 1328.58 & 1329.50 & 1331.27 \\ 1341.54 & 1364.00 & 1376.29 & 1380.75 & 1381.58 & 1391.21 \\ 1392.55 & 1469.54 & 1471.64 & 1472.54 & 1473.65 & 1474.00 \\ 1504.56 & 1525.81 & 1557.74 & 1559.07 & 1579.71 & 1602.12 \\ 1609.08 & 1609.46 & 1614.10 & 1614.86 & 1661.83 & 2545.31 \\ 3077.78 & 3087.94 & 3094.26 & 3104.91 & 3109.78 & 3117.11 \\ 3123.75 & 3123.82 & 3125.72 & 3127.34 & 3155.24 & 3155.91 \\ 3155.98 & 3159.09 & 3174.15 & 3174.89 & 3174.93 & 3177.98\end{array}$

TURBOMOLE Energies and Thermochemistry ${ }^{1}$

\begin{tabular}{|c|c|}
\hline RI-PBE/TZVP,SV(P) & -2955.367287 \\
\hline ZPE & 0.3784769 \\
\hline RI-PBE/TZVP,SV(P) + ZPE & -2954.98881 \\
\hline RI-PBE/TZVPP & -2956.92402 \\
\hline B3-LYP/TZVP,SV(P) & -2956.356317 \\
\hline ZPE (kJ/mol) & 993.7 \\
\hline In(qtrans) & 19.9 \\
\hline $\ln ($ qrot $)$ & 16.83 \\
\hline $\ln (q v i b)$ & 30.87 \\
\hline chem.pot. (kJ/mol) & 826.09 \\
\hline energy $(\mathrm{kJ} / \mathrm{mol})$ & 1068.36 \\
\hline entropy $(\mathrm{kJ} / \mathrm{mol} / \mathrm{K})$ & 0.82088 \\
\hline $\mathrm{Cv}(\mathrm{kJ} / \mathrm{mol}-\mathrm{K})$ & 0.4669177 \\
\hline $\mathrm{Cp}((\mathrm{kJ} / \mathrm{mol}-\mathrm{K})$ & 0.475232 \\
\hline $\mathrm{RI}-\mathrm{PBE} / \mathrm{TZVP}, \mathrm{SV}(\mathrm{P}) \quad<\mathrm{S}^{2}>$ & 1.13 \\
\hline $\mathrm{RI}-\mathrm{PBE} / \mathrm{TZVPP}<\mathrm{S}^{2}>$ & 1.12 \\
\hline B3 - LYP / TZVP, SV (P) $\quad<\mathrm{S}^{2}>$ & 1.64 \\
\hline
\end{tabular}

${ }^{1}$ Freeh output; $298.15 \mathrm{~K}, 0.1 \mathrm{MPa}$, unscaled 
${ }^{2}$ A Benzene:[(Porphyrin $\left.)(\mathrm{Fe}=\mathbf{O})(\mathrm{SH})\right]$ Transition State

RI-PBE/TZVP, SV(P) Cartesian coordinates

52

Energy $=-2955.348805644$

\begin{tabular}{|c|c|c|c|}
\hline C & -1.7903010 & -2.3814421 & 0.7519313 \\
\hline $\mathrm{N}$ & -0.5304624 & -1.8196913 & 0.8112846 \\
\hline C & 0.3355241 & -2.8567366 & 1.0714170 \\
\hline C & -0.3878113 & -4.1061148 & 1.1814191 \\
\hline $\mathrm{C}$ & -1.7122785 & -3.8089328 & 0.9813220 \\
\hline $\mathrm{Fe}$ & -0.0692293 & 0.1224418 & 0.5212943 \\
\hline 0 & -0.1259616 & -0.0047901 & -1.2036291 \\
\hline C & 1.7198615 & -2.7282575 & 1.2099403 \\
\hline C & 2.4160885 & -1.5240756 & 1.1129906 \\
\hline $\mathrm{N}$ & 1.8525682 & -0.2896811 & 0.8330160 \\
\hline $\mathrm{C}$ & 2.8982402 & 0.6197239 & 0.8961967 \\
\hline C & 4.1398158 & -0.0605607 & 1.1889749 \\
\hline C & 3.8404405 & -1.3930946 & 1.3220295 \\
\hline C & 2.7920398 & 1.9975897 & 0.7139012 \\
\hline C & 1.6019713 & 2.6826755 & 0.4516936 \\
\hline $\mathrm{N}$ & 0.3620329 & 2.1008273 & 0.3558429 \\
\hline $\mathrm{C}$ & -0.5165668 & 3.1209471 & 0.0622597 \\
\hline C & 0.1908755 & 4.3831184 & -0.0231271 \\
\hline C & 1.5125637 & 4.1106910 & 0.2237971 \\
\hline C & -1.8958098 & 2.9871356 & -0.1163653 \\
\hline $\mathrm{C}$ & -2.6083731 & 1.7901752 & -0.0204273 \\
\hline $\mathrm{N}$ & -2.0607608 & 0.5630459 & 0.2628218 \\
\hline C & -3.1016830 & -0.3311413 & 0.2678085 \\
\hline C & -4.3532281 & 0.3498480 & -0.0102293 \\
\hline C & -4.0453369 & 1.6730688 & -0.1916201 \\
\hline C & -2.9837906 & -1.7029105 & 0.5004226 \\
\hline S & -0.5403876 & 0.4103372 & 2.7621642 \\
\hline $\mathrm{H}$ & -0.9531093 & 1.7176070 & 2.7238675 \\
\hline $\mathrm{H}$ & -4.7178269 & 2.5105599 & -0.4209310 \\
\hline $\mathrm{H}$ & -5.3347848 & -0.1406325 & -0.0575509 \\
\hline $\mathrm{H}$ & -3.9076272 & -2.3010190 & 0.4791839 \\
\hline $\mathrm{H}$ & -2.5767576 & -4.4860772 & 0.9880341 \\
\hline $\mathrm{H}$ & 0.0749346 & -5.0802412 & 1.3891324 \\
\hline $\mathrm{H}$ & 2.2994670 & -3.6372847 & 1.4312222 \\
\hline $\mathrm{H}$ & 4.5145628 & -2.2285126 & 1.5537919 \\
\hline $\mathrm{H}$ & 5.1138029 & 0.4373757 & 1.2866537 \\
\hline $\mathrm{H}$ & 3.7172874 & 2.5898723 & 0.7814770 \\
\hline $\mathrm{H}$ & 2.3649449 & 4.8026125 & 0.2491211 \\
\hline $\mathrm{H}$ & -0.2818534 & 5.3494936 & -0.2444387 \\
\hline $\mathrm{H}$ & -2.4659038 & 3.9007869 & -0.3447791 \\
\hline C & 1.2132081 & -0.6058081 & -2.2557614 \\
\hline $\mathrm{C}$ & 1.2973167 & 0.4256934 & -3.2600167 \\
\hline C & 0.6880099 & -1.8881007 & -2.6546328 \\
\hline $\mathrm{C}$ & 0.1209077 & -2.0598255 & -3.9153465 \\
\hline C & 0.7294267 & 0.2385766 & -4.5152966 \\
\hline C & 0.1274482 & -0.9982084 & -4.8454940 \\
\hline $\mathrm{H}$ & 1.7679794 & 1.3852597 & -2.9908588 \\
\hline $\mathrm{H}$ & 0.7552671 & 1.0502563 & -5.2611566 \\
\hline $\mathrm{H}$ & -0.3267089 & -1.1375218 & -5.8406727 \\
\hline $\mathrm{H}$ & -0.32 & -3.0287777 & -4.1931282 \\
\hline $\mathrm{H}$ & 0.69 & -2.7114054 & -1.9221233 \\
\hline
\end{tabular}

$1.9653372-0.5910972-1.4482331$

$\begin{array}{rrrrrr}1408.87 & 0.00 & 0.00 & 0.00 & 0.00 & 0.00 \\ 0.00 & 21.40 & 28.93 & 39.20 & 47.37 & 51.28 \\ 78.63 & 89.10 & 98.52 & 101.14 & 113.45 & 146.28 \\ 147.87 & 155.84 & 191.08 & 206.19 & 207.48 & 232.61 \\ 256.19 & 270.13 & 273.08 & 275.88 & 280.75 & 296.71 \\ 297.03 & 323.53 & 349.62 & 354.23 & 359.12 & 370.75 \\ 380.03 & 396.57 & 397.45 & 413.33 & 419.15 & 420.31 \\ 422.00 & 423.01 & 460.14 & 583.49 & 585.84 & 595.28 \\ 631.74 & 648.14 & 652.54 & 657.48 & 658.73 & 673.84 \\ 683.12 & 695.72 & 696.72 & 699.47 & 718.13 & 719.67 \\ 723.89 & 734.61 & 736.19 & 763.49 & 771.10 & 771.39 \\ 778.04 & 783.51 & 785.05 & 785.95 & 798.43 & 799.14 \\ 838.41 & 847.01 & 848.35 & 855.43 & 872.83 & 885.63 \\ 890.59 & 891.38 & 893.78 & 945.61 & 949.69 & 966.20 \\ 972.63 & 990.90 & 999.71 & 1000.92 & 1009.27 & 1011.94 \\ 1012.36 & 1028.96 & 1030.27 & 1032.08 & 1032.52 & 1035.53 \\ 1040.12 & 1041.28 & 1041.79 & 1053.17 & 1131.79 & 1138.08 \\ 1140.08 & 1147.58 & 1148.31 & 1153.38 & 1181.96 & 1182.78 \\ 1260.09 & 1261.70 & 1322.54 & 1325.09 & 1329.00 & 1331.77 \\ 1343.51 & 1363.57 & 1369.09 & 1374.18 & 1381.46 & 1391.99 \\ 1397.36 & 1437.95 & 1459.54 & 1471.18 & 1471.70 & 1472.62 \\ 1505.44 & 1523.63 & 1547.62 & 1557.66 & 1559.00 & 1579.78 \\ 1595.55 & 1607.04 & 1610.32 & 1619.28 & 1659.76 & 2550.10 \\ 3075.62 & 3097.21 & 3105.84 & 3116.57 & 3121.54 & 3122.37 \\ 3123.49 & 3124.24 & 3124.59 & 3128.35 & 3153.00 & 3153.22 \\ 3153.76 & 3153.96 & 3172.35 & 3172.54 & 3172.87 & 3173.06\end{array}$

TURBOMOLE Energies and Thermochemistry ${ }^{1}$

\begin{tabular}{|c|c|}
\hline RI-PBE/TZVP,SV(P) & -2955.348806 \\
\hline ZPE & 0.3779309 \\
\hline RI-PBE/TZVP,SV(P) + ZPE & -2954.970875 \\
\hline RI-PBE/TZVPP & -2956.905233 \\
\hline B3-LYP/TZVP,SV(P) & -2956.331866 \\
\hline ZPE (kJ/mol) & 992.2 \\
\hline In(qtrans) & 19.9 \\
\hline $\ln ($ qrot $)$ & 16.65 \\
\hline $\ln (q v i b)$ & 23.78 \\
\hline chem.pot. (kJ/mol) & 842.71 \\
\hline energy $(\mathrm{kJ} / \mathrm{mol})$ & 1063.58 \\
\hline entropy $(\mathrm{kJ} / \mathrm{mol} / \mathrm{K})$ & 0.74909 \\
\hline $\mathrm{Cv}(\mathrm{kJ} / \mathrm{mol}-\mathrm{K})$ & 0.4604216 \\
\hline $\mathrm{Cp}((\mathrm{kJ} / \mathrm{mol}-\mathrm{K})$ & 0.4687359 \\
\hline $\mathrm{RI}-\mathrm{PBE} / \mathrm{TZVP}, \mathrm{SV}(\mathrm{P}) \quad<\mathrm{S}^{2}>$ & 0.92 \\
\hline $\mathrm{RI}-\mathrm{PBE} / \mathrm{TZVPP}<\mathrm{S}^{2}>$ & 0.92 \\
\hline B3 - LYP / TZVP, SV (P) $\quad<\mathrm{S}^{2}>$ & 1.47 \\
\hline
\end{tabular}

${ }^{1}$ Freeh output; $298.15 \mathrm{~K}, 0.1 \mathrm{MPa}$, unscaled 
${ }^{2}$ A Benzene: $[($ Porphyrin $)(\mathrm{Fe}=\mathbf{O})(\mathrm{SH})] \sigma-$ Complex

RI-PBE/TZVP, SV (P) Cartesian coordinates

52

Energy $=-2955.358743899$

\begin{tabular}{|c|c|c|c|}
\hline C & -1.7496614 & 2.6900333 & -0.5085319 \\
\hline $\mathrm{N}$ & -1.6378656 & 1.4483556 & 0.0764012 \\
\hline C & -2.9184279 & 1.0527473 & .3749778 \\
\hline C & -3.8722114 & 2.0652908 & .0359345 \\
\hline C & -3.1417940 & 3.0898429 & -0.5805666 \\
\hline $\mathrm{Fe}$ & 0.0753927 & 0.4169120 & 0.4193351 \\
\hline 0 & -0.0631904 & -0.3724106 & -1.2495383 \\
\hline $\mathrm{C}$ & -3.2621314 & -0.1388209 & 1.0191807 \\
\hline C & -2.3448285 & -1.0816847 & 1.4865208 \\
\hline $\mathrm{N}$ & -0.9696519 & -1.0188909 & 1.3214207 \\
\hline C & -0.4601262 & -2.1206467 & 1.9991538 \\
\hline C & -1.5339656 & -2.8897079 & 2.5815125 \\
\hline $\mathrm{C}$ & -2.7050926 & -2.2503217 & 2.2543446 \\
\hline C & 0.8871951 & -2.4697287 & 2.0857790 \\
\hline C & 1.9359699 & -1.7649160 & 1.4872016 \\
\hline $\mathrm{N}$ & 1.7980917 & -0.6007661 & 0.7699836 \\
\hline $\mathrm{C}$ & 3.0613476 & -0.2617305 & 0.342013 \\
\hline C & 4.0240696 & -1.2495869 & 0.787490 \\
\hline $\mathrm{C}$ & 3.3234045 & -2.1826714 & 1.5103221 \\
\hline $\mathrm{C}$ & 3.3918751 & 0.8895407 & -0.3761310 \\
\hline $\mathrm{C}$ & 2.4908938 & 1.8943527 & -0.7396009 \\
\hline $\mathrm{N}$ & 1.1390119 & 1.8876164 & -0.4829766 \\
\hline C & 0.6503518 & 3.0679394 & -0.9920566 \\
\hline C & 1.7216892 & 3.8491714 & -1.5771864 \\
\hline C & 2.8693034 & 3.1136418 & -1.4263362 \\
\hline C & -0.6916732 & 3.4586297 & -0.9935399 \\
\hline S & 0.3142984 & 1.4655464 & 2.4161813 \\
\hline $\mathrm{H}$ & 1.5269417 & 2.0617586 & 2.1795587 \\
\hline $\mathrm{H}$ & 3.8911041 & 3.3585114 & -1.7460034 \\
\hline $\mathrm{H}$ & 1.5913000 & 4.8318488 & -2.050028 \\
\hline $\mathrm{H}$ & -0.9356587 & 4.4355251 & -1.438638 \\
\hline $\mathrm{H}$ & -3.4968956 & 4.0398437 & -1.0023114 \\
\hline $\mathrm{H}$ & -4.9602474 & 1.9886324 & 0.0930055 \\
\hline $\mathrm{H}$ & -4.3288396 & -0.3270703 & 1.2142538 \\
\hline $\mathrm{H}$ & -3.7345574 & -2.5285487 & 2.5167416 \\
\hline $\mathrm{H}$ & -1.3952947 & -3.8090175 & 3.1660816 \\
\hline $\mathrm{H}$ & 1.1399087 & 3818271 & 2.6477008 \\
\hline $\mathrm{H}$ & 3.6977740 & -3.0814215 & 2.0185094 \\
\hline $\mathrm{H}$ & 5.1010441 & -1.2117316 & 0.5757424 \\
\hline $\mathrm{H}$ & 4.4491087 & 1.0323690 & -0.6472005 \\
\hline $\mathrm{C}$ & -0.4872831 & -1.6787388 & -1.573656 \\
\hline C & 0.6250608 & -2.6216581 & -1.9188892 \\
\hline C & -1.6021734 & -1.6699078 & -2.5692923 \\
\hline C & -1.6231525 & -2.5143492 & -3.663277 \\
\hline C & 0.5601457 & -3.4573872 & -3.0146497 \\
\hline C & -0.5572604 & -3.4217237 & -3.8952720 \\
\hline $\mathrm{H}$ & 1.4970410 & -2.6287510 & -1.2445190 \\
\hline $\mathrm{H}$ & 1.3927298 & -4.1488610 & -3.2278865 \\
\hline $\mathrm{H}$ & -0.5818310 & -4.0838198 & -4.7762208 \\
\hline $\mathrm{H}$ & -2.4677352 & -2.4763743 & -4.3718756 \\
\hline $\mathrm{H}$ & -2.411449 & -0.9437935 & -2.384824 \\
\hline $\mathrm{H}$ & -0 & -2.1107514 & -0.623298 \\
\hline
\end{tabular}

$\begin{array}{rrrrrr}0.00 & 0.00 & 0.00 & 0.00 & 0.00 & 0.00 \\ 18.05 & 21.57 & 39.02 & 45.24 & 50.67 & 80.31\end{array}$

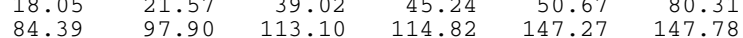

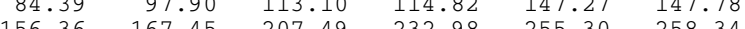
$\begin{array}{llllll}156.36 & 167.45 & 207.49 & 232.98 & 255.30 & 258.34\end{array}$ $\begin{array}{llllll}262.91 & 273.32 & 274.22 & 283.85 & 292.60 & 312.84 \\ 325.97 & 345.37 & 353.78 & 359.84 & 361.70 & 376.33\end{array}$ $\begin{array}{llllll}325.97 & 345.37 & 353.78 & 359.84 & 361.70 & 376.33\end{array}$ $\begin{array}{llllll}399.44 & 404.21 & 415.68 & 419.87 & 421.15 & 421.66\end{array}$ $\begin{array}{llllll}425.31 & 459.94 & 483.98 & 549.69 & 569.63 & 614.16\end{array}$ $\begin{array}{llllll}634.22 & 651.32 & 656.75 & 658.35 & 673.43 & 684.62\end{array}$ $\begin{array}{llllll}696.21 & 696.39 & 697.07 & 717.58 & 723.89 & 730.62\end{array}$ $\begin{array}{llllll}733.49 & 736.80 & 740.52 & 760.46 & 769.20 & 770.19\end{array}$ $\begin{array}{llllll}777.14 & 783.25 & 784.29 & 786.38 & 788.73 & 799.01\end{array}$ $\begin{array}{llllll}833.25 & 841.89 & 842.92 & 850.07 & 852.24 & 884.90\end{array}$ $\begin{array}{llllll}833.25 & 841.89 & 842.92 & 850.07 & 852.24 & 884.90 \\ 888.97 & 891.03 & 892.96 & 917.43 & 941.90 & 962.98\end{array}$ $\begin{array}{rrrrrr}888.97 & 891.03 & 892.96 & 917.43 & 941.90 & 962.98 \\ 989.19 & 991.47 & 999.54 & 1000.16 & 1005.87 & 1007.78\end{array}$ $\begin{array}{llllll}1009.79 & 1012.73 & 1029.90 & 1031.43 & 1034.91 & 1039.32\end{array}$ $\begin{array}{llllll}1040.72 & 1041.70 & 1053.33 & 1077.94 & 1120.77 & 1122.45\end{array}$ $\begin{array}{llllll}1138.28 & 1145.53 & 1146.19 & 1148.13 & 1181.86 & 1182.25\end{array}$ $\begin{array}{llllll}1259.25 & 1259.75 & 1261.46 & 1320.71 & 1327.96 & 1329.45\end{array}$ $\begin{array}{lllllll}1331.94 & 1344.73 & 1364.15 & 1366.92 & 1383.12 & 1394.90\end{array}$ 1331.94134 .730136 .1507 .960 $\begin{array}{llllll}1397.31 & 1402.69 & 1410.07 & 1467.33 & 1470.81 & 1473.49\end{array}$

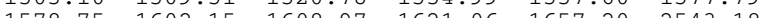
$\begin{array}{lllllll}1578.75 & 1602.15 & 1608.97 & 1621.06 & 1657.20 & 2543.18\end{array}$ $\begin{array}{llllll}2663.91 & 3093.48 & 3098.60 & 3117.07 & 3121.11 & 3121.81\end{array}$ $\begin{array}{llllll}3122.32 & 3122.41 & 3123.47 & 3126.17 & 3152.10 & 3152.90\end{array}$ $\begin{array}{llllll}3153.21 & 3154.08 & 3171.50 & 3172.25 & 3172.43 & 3172.91\end{array}$

TURBOMOLE Energies and Thermochemistry ${ }^{1}$ RI-PBE/TZVP,SV(P)

$-2955.358744$

ZPE

0.3774058

RI-PBE/TZVP,SV(P) + ZPE

RI-PBE/TZVPP

$-2954.981338$

B3-LYP/TZVP,SV(P)

ZPE $(\mathrm{kJ} / \mathrm{mol})$

$-2956.912134$

$\ln ($ qtrans)

$-2956.347526$

990.9

19.9

In(qrot)

16.66

In(qvib)

24.26

chem.pot. $(\mathrm{kJ} / \mathrm{mol})$

840.1

energy $(\mathrm{kJ} / \mathrm{mol})$

1062.75

entropy $(\mathrm{kJ} / \mathrm{mol} / \mathrm{K})$

0.7551

$\mathrm{Cv}(\mathrm{kJ} / \mathrm{mol}-\mathrm{K})$

0.4659763

$\mathrm{Cp}((\mathrm{kJ} / \mathrm{mol}-\mathrm{K})$

0.4742906

$\mathrm{RI}-\mathrm{PBE} / \mathrm{TZVP}, \mathrm{SV}(\mathrm{P})<\mathrm{S}^{2}>$

1.39

RI-PBE/TZVPP $\left\langle\mathrm{S}^{2}\right\rangle \quad 1.35$

B3 - LYP/TZVP, SV (P) $<\mathrm{S}^{2}>$

1.68

${ }^{1}$ Freeh output; $298.15 \mathrm{~K}, 0.1 \mathrm{MPa}$, unscaled

Vibrational frequencies 


\section{2,2,3,4,5,6-Hexachlorocyclohexadienone}

13

$\begin{array}{lrrr}\text { C } & -1.495763 & 0.338381 & 0.000052 \\ \text { C } & -1.327630 & -1.011811 & 0.000136 \\ \text { C } & 0.013425 & -1.624970 & -0.000063 \\ \text { C } & 1.243385 & -0.656277 & 0.000007 \\ \text { C } & 0.928049 & 0.815620 & -0.000036 \\ \text { C } & -0.348766 & 1.265327 & -0.000070 \\ \text { C1 } & 2.195140 & -1.058529 & -1.486465 \\ \text { Cl } & 2.277823 & 1.894342 & -0.000182 \\ \text { Cl } & -0.678861 & 2.967812 & 0.000129 \\ \text { C1 } & -3.085223 & 1.013521 & -0.000168 \\ \text { Cl } & -2.647430 & -2.123699 & 0.000000 \\ \text { O } & 0.196201 & -2.819116 & -0.000312 \\ \text { Cl } & 2.194680 & -1.058429 & 1.486824\end{array}$

Vibrational frequencies

$$
\begin{array}{r}
15.3180 \\
153.2062 \\
204.6488 \\
240.9193 \\
325.1297 \\
385.0035 \\
640.6085 \\
709.5349 \\
836.6847 \\
1144.0993 \\
1566.7884
\end{array}
$$

56.8343

176.4437

220.8748

273.6770

345.5384
456.4307

456.4307
651.0117

651.0117
747.4057

934.8682

180.1060

68.6443

183.7576

228.2801

312.3575

\begin{tabular}{|c|c|}
\hline B3LYP/6-31G* & -3064.96511284 \\
\hline ZPE & 0.045969 \\
\hline $\begin{array}{l}\text { Thermal Correction to } \\
\text { Enthalpy }\end{array}$ & 0.059805 \\
\hline $\begin{array}{l}\text { Thermal Correction to } \\
\text { Free Energy }\end{array}$ & 0.003563 \\
\hline Electronic State & $1-A$ \\
\hline Symmetry & $\mathrm{C} 1$ \\
\hline$\left\langle S^{\wedge} 2\right\rangle$ & 0.00 \\
\hline B3LYP/aug-cc-pVTZ ${ }^{2}$ & -3065.30296485 \\
\hline $\begin{array}{l}\mathrm{PCM}(\varepsilon=5.6)-B 3 L Y P / a u g- \\
\text { cc-pVTZ }^{2}\end{array}$ & -3065.30859797 \\
\hline $\begin{array}{l}\mathrm{PCM}(\varepsilon=78)-B 3 L Y P / a u g- \\
\text { cc-pVTZ }^{2}\end{array}$ & -3065.31172285 \\
\hline
\end{tabular}

376.3415

535.4113

685.9511

807.4206

1040.6772
1249.0480

1798.6011

Gaussian03 Energies and

Thermochemistry

${ }^{1} 298.15 \mathrm{~K}, 0.1 \mathrm{MPa}$, unscaled

${ }^{2}$ B3LYP/6-31G* Geometry 


\section{2,2,3,4,5,6-Hexachlorocyclohexadienone}

\section{Radical Anion}

\section{3}

\begin{tabular}{|c|c|c|c|}
\hline $\mathrm{C}$ & 1.371190 & 0.436164 & -0.157559 \\
\hline C & 1.216931 & -0.924233 & -0.321660 \\
\hline & -0.092979 & -1.543401 & -0.568684 \\
\hline & -1.226743 & -0.617999 & -0.42508 \\
\hline C & -1.045407 & 60913 & $-c$ \\
\hline C & 0.2 & 1.2 & $-c$ \\
\hline $\mathrm{Cl}$ & -0.845604 & -1.186727 & 16197 \\
\hline $\mathrm{Cl}$ & -2.433155 & 1.824761 & -0.269736 \\
\hline $\mathrm{Cl}$ & 0.461649 & 3.017742 & 0.031206 \\
\hline $\mathrm{Cl}$ & 2.968989 & 1.125856 & 0.045582 \\
\hline $\mathrm{Cl}$ & 2.578956 & -1.999687 & -0.379116 \\
\hline & -0.220496 & -2.717227 & -0.923221 \\
\hline & -2.790922 & -1.294799 & -0.729570 \\
\hline
\end{tabular}

Vibrational frequencies

$\begin{array}{rrr}25.5175 & 53.8726 & 69.6250 \\ 73.0194 & 96.8036 & 116.6018 \\ 186.5090 & 206.6992 & 215.4049 \\ 220.1229 & 233.9066 & 293.0211 \\ 313.9278 & 327.4579 & 342.4862 \\ 344.3493 & 371.2918 & 465.6802 \\ 543.9489 & 605.8781 & 651.8890 \\ 714.9292 & 721.2207 & 756.9539 \\ 846.0753 & 968.4969 & 1096.0868 \\ 1207.6500 & 1276.1602 & 1355.1162 \\ 1437.9820 & 1530.2438 & 1654.1135\end{array}$

Gaussian03 Energies and

Thermochemistry ${ }^{1}$

\begin{tabular}{|c|c|}
\hline B3LYP/6-31G* & -3065.06665614 \\
\hline ZPE & 0.044021 \\
\hline $\begin{array}{l}\text { Thermal Correction to } \\
\text { Enthalpy }\end{array}$ & 0.058763 \\
\hline $\begin{array}{l}\text { Thermal Correction to } \\
\text { Free Energy }\end{array}$ & -0.000403 \\
\hline Electronic State & $2-A$ \\
\hline Symmetry & C1 \\
\hline$\left\langle S^{\wedge} 2\right\rangle$ & 0.7673 \\
\hline B3LYP/aug-cc-pVTZZ ${ }^{2}$ & -3065.41495895 \\
\hline $\begin{array}{l}\mathrm{PCM}(\varepsilon=5.6)-\mathrm{B} 3 \mathrm{LYP} / \text { aug- } \\
\mathrm{cc}-\mathrm{pVTZ}{ }^{2}\end{array}$ & -3065.48108057 \\
\hline $\begin{array}{l}\mathrm{PCM}(\varepsilon=78)-\mathrm{B} 3 \mathrm{LYP} / \text { aug- } \\
\text { cc-pVTZ }\end{array}$ & -3065.50005564 \\
\hline
\end{tabular}

${ }^{1} 298.15 \mathrm{~K}, 0.1 \mathrm{MPa}$, unscaled

${ }^{2}$ B3LYP/6-31G* Geometry 
Pentachlorophenoxyl Radical (C2v)

12

$\begin{array}{lrrr}\mathrm{C} & 0.000000 & 1.246571 & -0.926893 \\ \mathrm{C} & 0.000000 & 1.237898 & 0.456807 \\ \mathrm{C} & 0.000000 & 0.000000 & 1.157942 \\ \mathrm{C} & 0.000000 & -1.237898 & 0.456807 \\ \mathrm{C} & 0.000000 & -1.246571 & -0.926893 \\ \mathrm{C} & 0.000000 & 0.000000 & -1.704747 \\ \mathrm{O} & 0.000000 & 0.000000 & -2.940755 \\ \mathrm{C} 1 & 0.000000 & -2.707119 & -1.832838 \\ \mathrm{C} 1 & 0.000000 & -2.727908 & 1.346965 \\ \mathrm{C} 1 & 0.000000 & 0.000000 & 2.880445 \\ \mathrm{C} 1 & 0.000000 & 2.727908 & 1.346965 \\ \mathrm{Cl} & 0.000000 & 2.707119 & -1.832838\end{array}$

Vibrational frequencies

$$
\begin{array}{r}
64.8945 \\
207.6812 \\
237.3789 \\
331.3720 \\
379.4195 \\
622.1810 \\
740.5833 \\
984.6707 \\
1254.4760 \\
1491.1372
\end{array}
$$

94.0298

218.4189

297.7983

349.6392

469.5678

662.2970

780.6777

1118.8433

1322.3335

1579.5425

Gaussian03 Energies and Thermochemistry

\begin{tabular}{ll}
\hline B3LYP/6-31G* & -2604.77675805 \\
\hline ZPE & 0.044021 \\
Thermal Correction to & 0.055962 \\
$\begin{array}{l}\text { Enthalpy } \\
\text { Thermal Correction to }\end{array}$ & 0.004509 \\
$\begin{array}{l}\text { Free Energy } \\
\text { Electronic State }\end{array}$ & $2-B 1$ \\
Symmetry & C2V \\
\hline$<S^{\wedge} 2>$ & 0.7856 \\
\hline B3LYP/aug-cc-pVTZ & -2605.07510174 \\
\hline PCM( $\varepsilon=5.6)-B 3 L Y P / a u g-$ & -2605.08142466 \\
cc-pVTZ & \\
\hline PCM $(\varepsilon=78)-B 3 L Y P / a u g-$ & -2605.08451255 \\
cc-pVTZ & \\
\hline
\end{tabular}

${ }^{1} 298.15 \mathrm{~K}, 0.1 \mathrm{MPa}$, unscaled

${ }^{2}$ B 3 LYP / 6-31G* Geometry 


$\begin{array}{lrrr}\text { C } & 0.000000 & 1.211595 & -0.934273 \\ \text { C } & 0.000000 & 1.207582 & 0.454570 \\ \text { C } & 0.000000 & 0.000000 & 1.178961 \\ \text { C } & 0.000000 & -1.207582 & 0.454570 \\ \text { C } & 0.000000 & -1.211595 & -0.934273 \\ \text { C } & 0.000000 & 0.000000 & -1.745666 \\ \text { O } & 0.000000 & 0.000000 & -2.989120 \\ \text { C1 } & 0.000000 & -2.717547 & -1.836460 \\ \text { C1 } & 0.000000 & -2.731356 & 1.340915 \\ \text { C1 } & 0.000000 & 0.000000 & 2.936362 \\ \text { C1 } & 0.000000 & 2.731356 & 1.340915 \\ \text { Cl } & 0.000000 & 2.717547 & -1.836460\end{array}$

Vibrational frequencies

$\begin{array}{rrr}63.0586 & 70.8307 & \\ 165.9895 & 203.6293 & 211.6197 \\ 217.4920 & 229.3882 & 289.1507 \\ 298.3182 & 319.7311 & 327.5911 \\ 336.3199 & 366.5081 & 457.7497 \\ 558.8685 & 600.4812 & 639.1423 \\ 709.6997 & 737.4056 & 738.2897 \\ 830.7863 & 964.6907 & 1085.0240 \\ 1192.2280 & 1291.2752 & 1420.2318 \\ 1470.1623 & 1552.3471 & 1669.5067\end{array}$

Gaussian03 Energies and Thermochemistry

\begin{tabular}{ll}
\hline B3LYP/6-31G* & -2604.88720109 \\
\hline ZPE & 0.043552 \\
$\begin{array}{l}\text { Thermal Correction to } \\
\text { Enthalpy }\end{array}$ & 0.056019 \\
$\begin{array}{l}\text { Thermal Correction to } \\
\text { Free Energy }\end{array}$ & 0.004827 \\
$\begin{array}{l}\text { Electronic State } \\
\text { Symmetry }\end{array}$ & $1-\mathrm{A} 1$ \\
\hline$<\mathrm{S}^{\wedge} 2>$ & $\mathrm{C} 2 \mathrm{~V}$ \\
\hline B3LYP/aug-cc-pVTZ & 0.0000 \\
\hline PCM $(\varepsilon=5.6)-B 3 L Y P / a u g-$ & -2605.19715007 \\
cc-pVTZ & -2605.25609037 \\
\hline $\begin{array}{ll}\text { PCM }(\varepsilon=78)-B 3 L Y P / a u g- \\
\text { cc-pVTZ }\end{array}$ & -2605.27015499 \\
\hline
\end{tabular}

${ }^{1} 298.15 \mathrm{~K}, 0.1 \mathrm{MPa}$, unscaled

${ }^{2} \mathrm{~B} 3 \mathrm{LYP} / 6-31 \mathrm{G}$ * Geometry 


\section{1,2-Tetrachloroquinone}

12

$\begin{array}{lrrr}\mathrm{C} & 0.000000 & 1.458847 & 0.416300 \\ \mathrm{C} & 0.000000 & 0.776939 & 1.732272 \\ \mathrm{C} & 0.000000 & -0.776939 & 1.732272 \\ \mathrm{C} & 0.000000 & -1.458847 & 0.416300 \\ \mathrm{C} & 0.000000 & -0.741307 & -0.739575 \\ \mathrm{C} & 0.000000 & 0.741307 & -0.739575 \\ \mathrm{C} 1 & 0.000000 & -3.183181 & 0.457552 \\ \mathrm{C} 1 & 0.000000 & 1.549626 & -2.267169 \\ \mathrm{C} 1 & 0.000000 & 3.183181 & 0.457552 \\ \mathrm{O} & 0.000000 & 1.368403 & 2.788687 \\ \mathrm{C} 1 & 0.000000 & -1.549626 & -2.267169 \\ \mathrm{O} & 0.000000 & -1.368403 & 2.788687\end{array}$

Vibrational frequencies

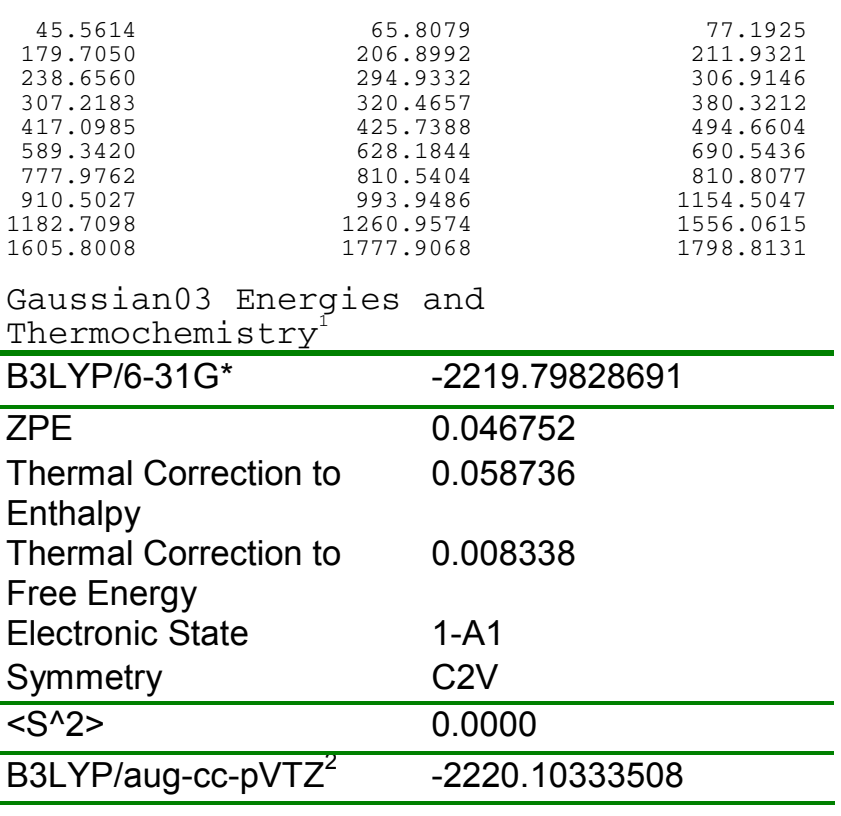

${ }^{1} 298.15 \mathrm{~K}, 0.1 \mathrm{MPa}$, unscaled

${ }^{2}$ B3 LYP / 6-31G* Geometry 


\section{1,4-Tetrachloroquinone}

12

$\begin{array}{lrrr}\text { C } & 0.000000 & 0.675454 & -1.279776 \\ \text { C } & 0.000000 & -0.675454 & -1.279776 \\ \text { C } & 0.000000 & -1.458997 & 0.000017 \\ \text { C } & 0.000000 & -0.675533 & 1.279646 \\ \text { C } & 0.000000 & 0.675533 & 1.279646 \\ \text { C } & 0.000000 & 1.458997 & 0.000017 \\ \text { C1 } & 0.000000 & -1.618280 & 2.719726 \\ \text { C1 } & 0.000000 & 1.618749 & -2.719547 \\ \text { C1 } & 0.000000 & 1.618280 & 2.719726 \\ \text { O } & 0.000000 & -2.671776 & -0.000295 \\ \text { C1 } & 0.000000 & -1.618749 & -2.719547 \\ \text { O } & 0.000000 & 2.671776 & -0.000295\end{array}$

Vibrational frequencies

$\begin{array}{rrr}53.1908 & 62.2889 & 86.9358 \\ 179.7033 & 198.5916 & 204.4619 \\ 212.8517 & 265.2370 & 307.1550 \\ 324.8250 & 339.3179 & 379.6248 \\ 431.7557 & 467.3416 & 488.6200 \\ 555.5192 & 730.5656 & 731.1931 \\ 736.9177 & 770.2429 & 850.4589 \\ 909.2857 & 994.5070 & 1112.1856 \\ 1231.5640 & 1246.7897 & 1608.8576 \\ 1647.8370 & 1775.3222 & 1779.1754\end{array}$

Gaussian03 Energies and

Thermochemistry

\begin{tabular}{ll}
\hline B3LYP/6-31G* $^{*}$ & -2219.81259049 \\
\hline ZPE & 0.047118 \\
Thermal Correction to & 0.059064 \\
Enthalpy & \\
Thermal Correction to & 0.008788 \\
Free Energy & \\
Electronic State & $1-\mathrm{A} 1$ \\
Symmetry & $\mathrm{C} 2 \mathrm{~V}$ \\
\hline$<\mathrm{S}^{\wedge} 2>$ & 0.0000 \\
\hline B3LYP/aug-cC-pVTZ & -2220.10333508 \\
\hline
\end{tabular}

${ }^{1} 298.15 \mathrm{~K}, 0.1 \mathrm{MPa}$, unscaled

${ }^{2}$ B3LYP/6-31G* Geometry 